\title{
AERODYNAMIC STUDY OF TILTING ASYMMETRICAL DUCTED FANS MOUNTED AT THE WING TIPS OF A VTOL UAV
}

\author{
by \\ Bahram Raeisi \\ Master of Engineering (Ryerson University, Toronto) 2011 \\ B.A.Sc. (Amirkabir University of Technology, Tehran) 1992
}

\author{
A dissertation \\ presented to Ryerson University \\ in partial fulfillment of the \\ requirements for the degree of \\ Doctor of Philosophy \\ in the Program of \\ Aerospace Engineering
}

Toronto, Ontario, Canada, 2016

(C) Bahram Raeisi 2016 
I hereby declare that I am the sole author of this dissertation. This is a true copy of the dissertation, including any required final revisions, as accepted by my examiners.

I authorize Ryerson University to lend this dissertation to other institutions or individuals for the purpose of scholarly research.

I further authorize Ryerson University to reproduce this dissertation by photocopying or by other means, in total or in part, at the request of other institutions or individuals for the purpose of scholarly research.

I understand that my dissertation may be made electronically available to the public. 


\title{
AERODYNAMIC STUDY OF TILTING ASYMMETRICAL DUCTED FANS MOUNTED AT THE WING TIPS OF A VTOL UAV
}

\author{
Bahram Raeisi \\ Doctor of Philosophy, Aerospace Engineering, Ryerson University, 2016
}

\begin{abstract}
Tilting ducted fans attached to the wing tips of vertical take-off and landing unmanned aerial vehicles (VTOL UAVs) define new applications for these types of vehicles. This new configuration gives VTOL UAVs the ability to hover like helicopters and fly forward like airplanes. These abilities provide VTOL UAVs with possibility of using any arbitrary location for take-off and landing combined with enhanced range and speed. The thrust vectoring is another advantage of this new configuration, which can be used in most of the necessary maneuvers. The flow behaviour around tilting ducted fans needs to be studied as it has significant effects on the performance of the VTOL UAVs. The first objective of this research is to investigate the use of asymmetrical shape for the external body of ducted fans. This geometry can generate additional lift in cruise mode, which can lead to more applications for the VTOL UAVs by saving more energy. Both CFD and experimental methods showed noticeable improvement in the lift coefficient by using an asymmetrical ducted fan. The second objective is to predict the induced velocity $\left(V_{\text {ind }}\right)$ of the inlet flow to the rotor plane. This is required for computing aerodynamic coefficients necessary for stability and control analysis of the proposed VTOL UAV. "Actuator Disk Model" combined with the assumption of "Constant Delivered Power" to the propeller were used successfully to calculate $V_{\text {ind }}$ for the CFD simulations. The third objective is using CFD
\end{abstract}


simulation for predicting aerodynamic forces and pitching moments of the tilting ducted fans in the transition conditions for different tilting rates. The effects of the stall and flow separation on the aerodynamic coefficients were discussed and compared for both ducted fans. The fourth objective is using the aerodynamic coefficients of the tilting ducted fans to predict and compare the level flight conditions of the proposed VTOL UAV during transition between cruise mode and hover. Results of this research demonstrate satisfactory agreement between CFD simulations and wind tunnel tests for all of these objectives, which could predict the aerodynamic behaviour of the proposed VTOL UAV during transition between cruise mode and hover. 


\section{ACKNOWLEDGEMENTS}

I would like to thank Dr. Hekmat Alighanbari for supporting and advising me during my research and analysis. His knowledge, guidance and support made my thesis work possible.

I also gratefully acknowledge Dr. Hamid Ghaemi, Jerry Karpynczyk, and Peter Bradley for their outstanding technical support.

My parents, Ahmad and Nazdar deserve my highest respect and deepest gratitude for their constant support and encouragement in all the steps that I took in my life.

And finally, I would like to express my deepest thanks and gratitude to my wife Nazi, for her endless emotional support, extensive help, patience, support and encouragement throughout the long process of my Ph.D. thesis. 


\section{TABLE OF CONTENTS}

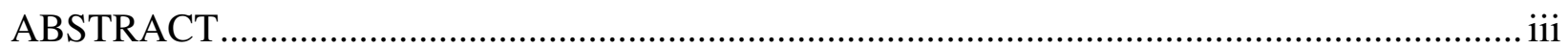

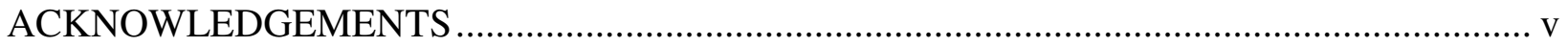

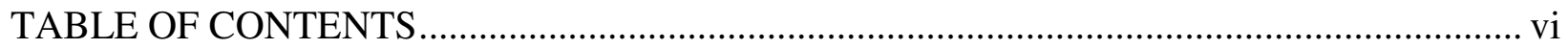

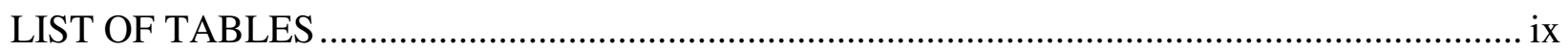

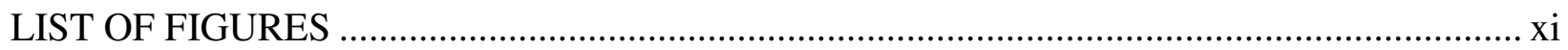

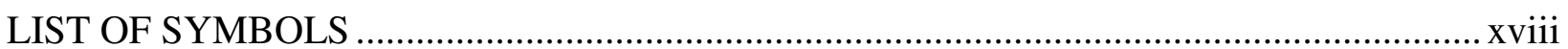

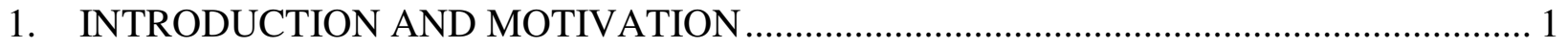

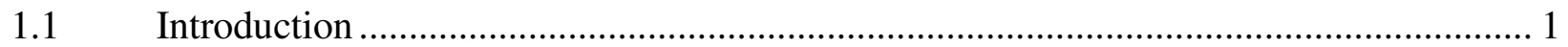

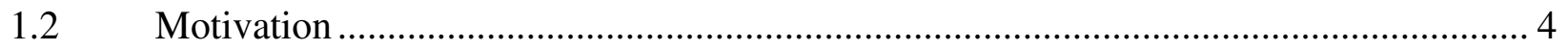

2. BACKGROUND AND LITERATURE REVIEW ........................................................ 8

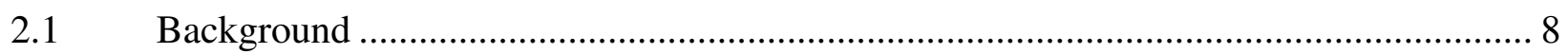

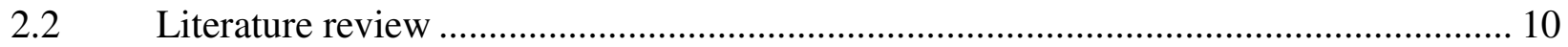

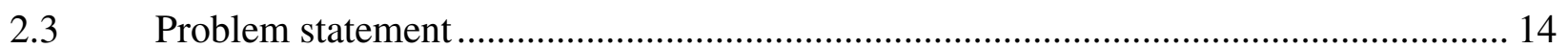

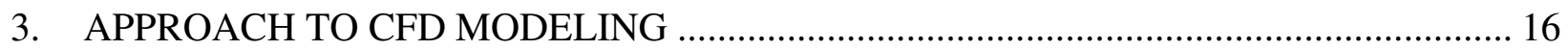

3.1 "Momentum Theory" and "Actuator Disk Model" .................................................. 16

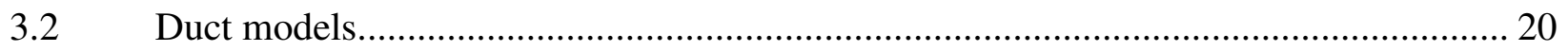

3.3 Governing equation, CFD code, mesh generation and computational domain............ 22

3.4 Defining aerodynamic forces and pitching moment acting on the models .................. 23

3.5 Turbulence modeling for ducted fans in crosswind ......................................... 26

3.6 Mesh selection for CFD modeling ................................................................. 27

3.7 Proposed method to calculate the inlet flow velocity to the ducted fans ..................... 29

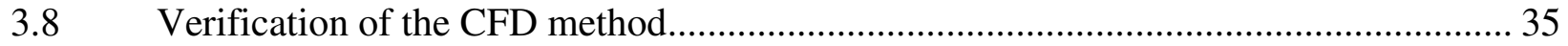




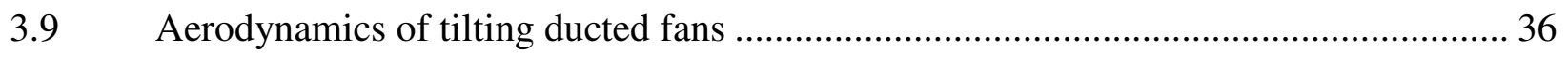

3.10 Tilting ducted fan VTOL UAV in transition condition............................................. 37

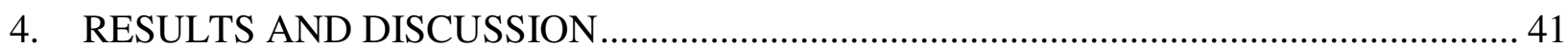

4.1 CFD simulations for symmetrical and asymmetrical ducts without fans....................... 41

4.2 Experimental validation of the CFD results for ducts without fans .............................. 45

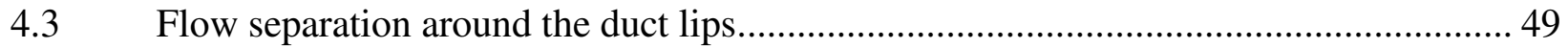

4.4 The CFD simulations for symmetrical and asymmetrical ducted fans .......................... 50

4.5 Aerodynamic coefficients for ducted fans by CFD modeling ......................................... 54

4.6 Experimental validation of the CFD results for ducted fans ........................................ 55

4.7 Aerodynamic coefficients for ducted fans from wind tunnel experiments ..................... 60

4.8 Tilting ducted fans attached to the wing tips of proposed VTOL UAV in transition .... 61

4.9 The effects of changes in the tilting rate of ducted fans in transition mode ...................64 64

4.10 Proposed VTOL UAV in the transition from cruise mode to hover ............................... 67

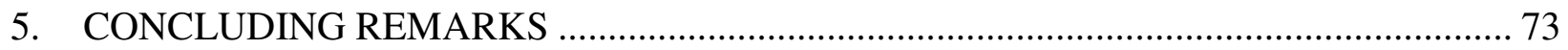

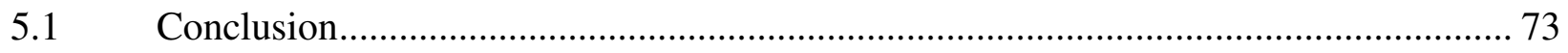

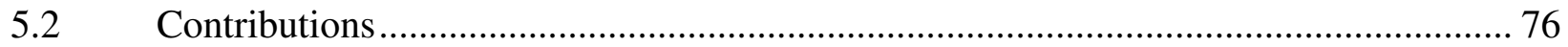

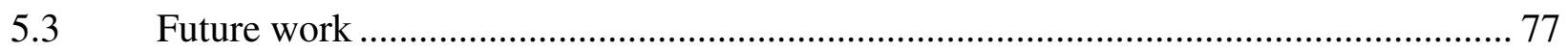

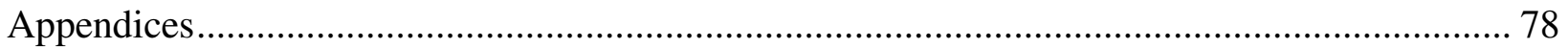

Appendix A: More about the turbulence modeling ……............................................................. 79

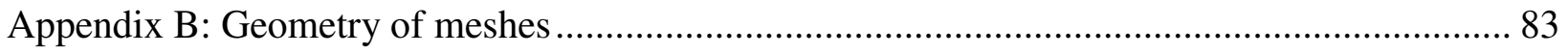

Appendix C: Designing, producing, and preparing actual models of ducted fans ........................ 86

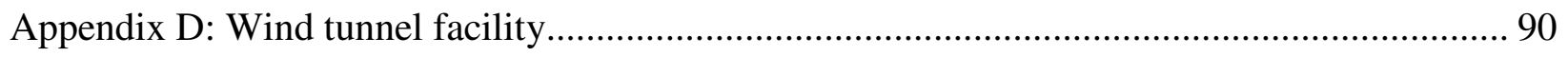

Appendix E: Measuring aerodynamic forces on the frame and ducts with/without fans ............. 92 
Appendix F: Measuring pitching moments of the ducts' weights

Appendix G: Error analysis for measuring aerodynamic coefficients

Appendix H: Summary of ANSYS-Fluent settings in the transient mode and User Defined

Functions

Appendix I: The specifications of the proposed VTOL UAV with two ducted fans

Appendix J: MATLAB code for calculating flight parameters of proposed VTOL UAV during

transition between cruise mode to hover

REFERENCES 


\section{LIST OF TABLES}

Table 2.1: List of V/STOL aircraft from 1950s [7] .............................................................. 8

Table 3.1: The specifications of different meshes of symmetrical duct without fan including No.

of volumetric cells and CPU time per step for computing lift coefficient................................ 27

Table 3.2: Number of volumetric cells for different mesh sizes in this research ..................... 29

Table 3.3: Specifications of symmetrical and asymmetrical ducted fans ................................ 31

Table 3.4: Initial setup for the ANSYS-Fluent software with models in zero AoA .................. 31

Table 3.5: Final induced velocities, thrust and induced power for each duct at zero AoA ......... 32

Table 3.6: Calculated Vind and Thrust for symmetrical and asymmetrical ducted fans .......... 34

Table 4.1: Final setup for the ANSYS-Fluent software ................................................. 51

Table 4.2: Important parameters for both ducted fans in cruise mode $\left(U=15 \mathrm{~m} / \mathrm{s} \& A o A=0^{\circ}\right)$

Table 4.3: The final travelled time for transition from cruise mode to hover for proposed VTOL

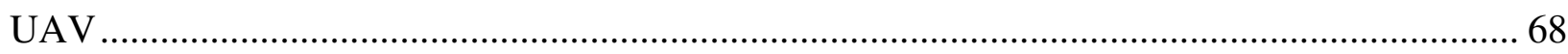

Table 4.4: Maximum tilting angle of ducted fans in the transition from cruise mode to hover ... 69

Table B. 1: Number of spacing and interval size for different edges of the meshes for symmetrical and asymmetrical ducts

Table B. 2: Number of spacing and interval size for different edges of additional components (rotor and shaft)

Table B. 3: Number of volumetric tetrahedron cells for different models of ducts with/without

fans 
Table G. 1: Error of reading for different quantities during experiments in the wind tunnel...... 97

Table G. 2: Maximum error for each aerodynamic coefficient in the different experiment ........ 98

Table I. 1: Specifications of the proposed VTOL UAV equipped with two symmetrical (or by

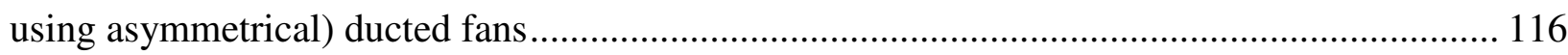




\section{LIST OF FIGURES}

Figure 1-1: Yamaha R-Max helicopter (Public domain [1]) .................................................. 2

Figure 1-2: Comparison between streamlines into (a) an open propeller and (b) a ducted fan in a

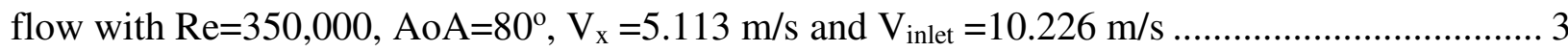

Figure 1-3: The most recent ducted fan UAVs: (a) Honeywell T-Hawk (Public domain [3]), (b)

BAE Systems IAV2 (Public domain [4]), (c) Aurora Golden Eye-50 (Public domain [5]) .......... 3

Figure 1-4: The generated gyroscopic moment of $\operatorname{IR} \phi \theta$ (about z-axis) on tilting ducted fan ....... 5

Figure 1-5: Schematic of the the proposed VTOL UAV with two tilting ducted fans installed on

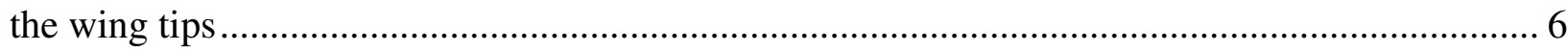

Figure 3-1: The ideal actuator disk and flow in the slipstream......................................... 17

Figure 3-2: Ducted fan and flow model for using momentum theory .................................... 19

Figure 3-3: Symmetrical ducted fan with NACA0012 profile for external body: (a) 3D view, (b)

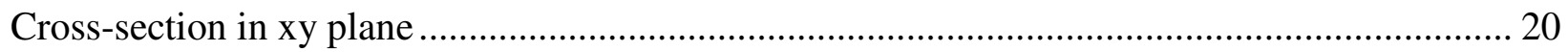

Figure 3-4: (a) Locations of different profiles of the high lift FX63-137 airfoil on the different sides of the external body of asymmetrical ducted fan, (b) Front view, (c) 3D view, and (d)

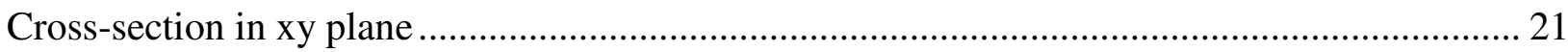

Figure 3-5: (a) Computational domain and (b) interior border around the duct ....................... 22

Figure 3-6: Comparing the size of cells for (a) whole domain, and (b) around the ducted fan .... 23

Figure 3-7: Contour of pressure distribution and the resultant aerodynamic forces and pitching

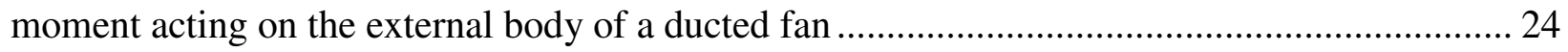
Figure 3-8: Comparison of aerodynamic coefficients for different mesh sizes for symmetrical

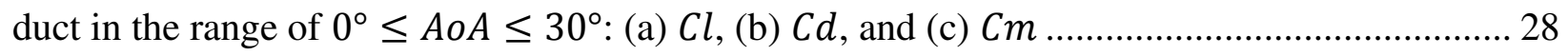


Figure 3-9: Comparing $\mathrm{Cl}$ for seven meshes with different No. of cells in stationary state ( $\mathrm{AoA}=$ $\left.30^{\circ}, U x=15 \mathrm{~m} / \mathrm{sec}, \operatorname{Re}=226,000\right)$ 29

Figure 3-10: The flowchart for finding power in cruise mode and also Vind in all different AoAs for each ducted fan. 33

Figure 3-11: Variations of induced velocity (Vind) and thrust (T) in the range of $-30^{\circ} \leq \alpha \leq$ $120^{\circ}$ for symmetrical and asymmetrical ducted fans with assumption of $P=$ const. 34

Figure 3-12: Duct wake bending with increasing crossflow. Progression is from 0 to 30 knots (equal to 0 to $15.43 \mathrm{~m} / \mathrm{s}$ ), $12 \mathrm{psf}$ disks loading modeled (equal to $574.6 \mathrm{~N} / \mathrm{m}^{2}$ or $15.314 \mathrm{~m} / \mathrm{s}$ of inlet flow velocity to the duct). Range of color contours is 0 to $136 \mathrm{ft} / \mathrm{sec}$. [9] 35

Figure 3-13: Duct wake bending with increasing crossflow in this research. Progression is from 0 to 30 knots (equal to 0 to $15.43 \mathrm{~m} / \mathrm{s}$ ), $12 \mathrm{psf}$ disk loading modeled (equal to $574.6 \mathrm{~N} / \mathrm{m}^{2}$ or $15.314 \mathrm{~m} / \mathrm{s}$ of inlet flow velocity to the duct). Range of color contours is 0 to $40 \mathrm{~m} / \mathrm{sec}$. 35

Figure 3-14: Flow streamlines combined with contour of vorticity on the xy plane around the symmetrical ducted fan 36

Figure 3-15: Coordinate system and aerodynamic forces acting on the proposed typical VTOL UAV

Figure 4-1: Two ducts without fans; symmetrical duct: (a) front view, (b) cross section in the xy plane and asymmetrical duct: (c) front view, (d) cross section in the xy plane...... 41

Figure 4-2: The variation of aerodynamic coefficients for symmetrical and asymmetrical ducts without fans (a) $\mathrm{Cl}$, (b) $\mathrm{Cd}$, and (c) $\mathrm{Cm}$

Figure 4-3: The contour of pressure around the symmetrical duct (top) and asymmetrical duct (bottom) without fans in steady flow $\left(\operatorname{Re}=226,000 \& A o A=0^{\circ}, 30^{\circ}, 60^{\circ} \& 90^{\circ}\right)$.............. 
Figure 4-4: The contour of pressure on the half-body ducts (without fans) and plane of symmetry at $A o A=45^{\circ}$; (a) symmetrical duct, (b) asymmetrical duct 44

Figure 4-5: The symmetrical and asymmetrical ducts installed in the wind tunnel 45

Figure 4-6: The mechanism used for measuring experimental pitching moment in the wind tunnel. 45

Figure 4-7: Comparison of (a) lift, (b) drag and (c) pitching moment coefficients from experiments with CFD for symmetrical duct without fan in $\mathrm{Re}=226,000$ 46

Figure 4-8: Comparison of (a) lift, (b) drag, and (c) pitching moment coefficients from experiments with CFD for asymmetrical duct without fan in $\mathrm{Re}=226,000$ 48

Figure 4-9: Comparing experimental (a) lift, (b) drag, and (c) pitching moment coefficients of symmetrical with asymmetrical ducts without fan, $\mathrm{Re}=226,000$ 49

Figure 4-10: Two ducted fans; symmetrical duct: (a) front view, (b) cross section in xy plane and asymmetrical duct: (c) front view, (d) cross section in $x y$ plane 50

Figure 4-11: The variation of aerodynamic forces and pitching moments for symmetrical and asymmetrical ducted fans in $\mathrm{Re}=226,000$; (a) Fy, (b) Fx, and (c) $M o$. 52 Figure 4-12: The contour of pressure around symmetrical ducted fan (top) and asymmetrical ducted fan (bottom) in steady flow $\left(U=15 \mathrm{~m} / \mathrm{s} \& A o A=0^{\circ}, 30^{\circ}, 60^{\circ} \$ 90^{\circ}\right)$ 53 Figure 4-13: The contour of pressure on the half-body ducted fans and plane of symmetry at $A o A=45^{\circ} ;$ (a) symmetrical ducted fan, (b) asymmetrical ducted fan

Figure 4-14: The variation of aerodynamic coefficients for symmetrical and asymmetrical ducted fans under the influence of active fans in different AoA (a) $C l$, (b) $C d$, and (c) $C m, \operatorname{Re}=226,000$. 
Figure 4-15: The symmetrical and asymmetrical ducted fans installed in the wind tunnel and the mechanism used for measuring experimental pitching moment in the wind tunnel...... 56

Figure 4-16: The variation of resultant forces and pitching moments for symmetrical ducted fan in $\mathrm{Re}=226,000$; (a) Fy, (b) Fx, and (c) Mo. 57

Figure 4-17: The variation of resultant forces and pitching moments for asymmetrical ducted fan in $\mathrm{Re}=226,000$; (a) $F y$, (b) $F x$, and (c) $M o$. 59

Figure 4-18: Comparing experimental resultant forces and pitching moments of symmetrical and asymmetrical ducted fans, $\operatorname{Re}=226,000$; (a) Fy, (b) $F x$, and (c) Mo. 60

Figure 4-19: The variation of experimental aerodynamic coefficients for symmetrical and asymmetrical ducted fans under the influence of active fans in different AoA; (a) $C l$, (b) $C d$, and (c) $C m, \operatorname{Re}=226,000$. 61

Figure 4-20: Comparing aerodynamic coefficients for symmetrical and asymmetrical ducted fans in transition from $\alpha=-30^{\circ}$ to $\alpha=120^{\circ}$ with $\omega=10 \mathrm{deg} / \mathrm{sec}$ and $U x=15 \mathrm{~m} / \mathrm{s}$; (a) $\mathrm{Cl}$, (b) $C d$ and (c) $C m$ 63 Figure 4-21: Comparing resultant forces and moments on symmetrical and asymmetrical ducted fans in transition from $\alpha=-30^{\circ}$ to $\alpha=120^{\circ}$ with $\omega=10 \mathrm{deg} / \mathrm{sec}$ and $U x=15 \mathrm{~m} / \mathrm{s}$; (a) Fy, (b) $F x$ and (c) $M o$ 64

Figure 4-22: The effects of changes in the tilting rate on the transitional aerodynamic coefficients for symmetrical and asymmetrical ducted fans: (a \& d) $C l$, (b \& e) $C d$ and (c \& f) $C m$ in three different tilting rates: $\omega=10,20 \& 30 \mathrm{deg} / \mathrm{sec}, 30^{\circ} \leq \alpha \leq 120^{\circ}$, and $U x=15 \mathrm{~m} / \mathrm{s}$

Figure 4-23: The effects of tilting rate changes on the transitional resultant forces and pitching moment of the symmetrical and asymmetrical ducted fans: (a \& d) $C l$, (b \& e) $C d$ and (c \& f) $\mathrm{Cm}$ in three different rates: $\omega=10,20 \& 30 \mathrm{deg} / \mathrm{sec}, 30^{\circ} \leq \alpha \leq 120^{\circ}$, and $U x=15 \mathrm{~m} / \mathrm{s} . .67$ 
Figure 4-24: The variation of tilting angle of the ducted fans during transition for symmetrical and asymmetrical ducted fans in three different tilting rates $(\omega=10,20 \& 30 \mathrm{deg} / \mathrm{sec})$........ 68

Figure 4-25: The variation of VTOL UAV's travelled distance during transition by using symmetrical and asymmetrical ducted fans in different tilting rates $(\omega=10,20 \& 30 \mathrm{deg} / \mathrm{sec})$

Figure 4-26: The variation of VTOL UAV's velocity during transition by using symmetrical and asymmetrical ducted fans in three different tilting rates $(\omega=10,20 \& 30 \mathrm{deg} / \mathrm{sec})$ 70

Figure 4-27: The variation of VTOL UAV's x-acceleration during transition by using symmetrical and asymmetrical ducted fans in different tilting rates $(\omega=10,20 \& 30 \mathrm{deg} / \mathrm{sec})$

Figure 4-28: The variation of ducted fans thrust (for one engine) during transition by using symmetrical and asymmetrical ducted fans in different tilting rates $(\omega=10,20 \& 30 \mathrm{deg} / \mathrm{sec})$

Figure 4-29: The changes in the pitching moments during transition by using symmetrical and asymmetrical ducted fans in three different tilting rates $(\omega=10,20 \& 30 \mathrm{deg} / \mathrm{sec})$ 72

Figure A. 1: Comparison between different turbulence modeling and experimental results of symmetrical duct without fan; (a) lift, (b) drag.... 79

Figure A. 2: Comparison between standard and RNG $k-\varepsilon$ turbulence modeling with experimental results of symmetrical ducted fan; (a) Vertical forces, $F y$, (b) Horizontal forces, $F x$

Figure C. 1: Designs of models by CATIA 5; (a) Symmetrical duct, (b) Asymmetrical duct ..... 86 
Figure C. 2: Asymmetrical duct before smoothing the surface

Figure C. 3: Two ducts after installation of electromotor and 8x6" 3-bladed propeller: (a) symmetrical ducted fan (b) asymmetrical ducted fan

Figure C. 4: (a) Supporting frame, and (b) Gear system for tilting ducts 88

Figure C. 5: Devices for controlling propeller's RPM and duct's tilting motion 88

Figure C. 6: Installation of speed controller in the test section at the back of supporting frame . 89

Figure D. 1: (a) The "Test Section" and (b) the main control unit 90

Figure D. 2: The manometers for measuring air velocity in the test section 91

Figure D. 3: (a) The balancing device for hanging the model and measuring forces and pitching moments, (b) the display for measured forces and pitching moment 91

Figure F. 1: Pitching moments due to the weight of ducts without fans; (a) symmetrical duct, (b) asymmetrical duct 95

Figure F. 2: Pitching moments due to the weight of ducted fans; (a) symmetrical ducted fan, (b) asymmetrical ducted fan 96

Figure G. 1: Error limits for experimental aerodynamic coefficients of ducts without fans; (a), (b), and (c) are error limits on the $C l, C d$, and $C m$ of symmetrical duc, (d), (e), and (f) are error limits on the $C l, C d$, and $C m$ of asymmetrical duct. 99 Figure G. 2: Error limits for experimental aerodynamic coefficients of ducted fans; (a), (b), and (c) are error limits on the $C l, C d$, and $C m$ of symmetrical ducted fan, (d), (e), and (f) are error limits on the $C l, C d$, and $C m$ of asymmetrical ducted fan. 100 
Figure I.1: Proposed tilting ducted fan with airfoil shape fuselage 


\section{LIST OF SYMBOLS}

\section{Abbreviations}

AoA Angle of attack (angle between main flow direction and centre line of the flying object)

UAV Unmanned Aerial Vehicle

VTOL Vertical Take-off and Landing

\section{Parameters}

A Area of the rotor disk

$a_{w} \quad$ Wake contraction parameter

c Chord

$C_{d} \quad$ Drag coefficient

$C_{l} \quad$ Lift coefficient

$C_{m} \quad$ Pitching moment coefficient

D Drag

$\mathrm{D}_{\text {ram }}$ Ram drag

$g \quad$ Gravitational acceleration

h Moment's arm

$h \quad$ Height of water in the manometer (inch of water)

hs Horizontal stabilizer

$\mathrm{h}_{\mathrm{ac}} \quad$ Vertical distance of wing's ac to the $\mathrm{cg}$

$\overline{\mathrm{h}}_{\mathrm{cg}} \quad$ Horizontal distance from the tilting axis to the centre of gravity of the duct

$\mathrm{h}_{\text {ram }}$ Distance between fan's centre of pressure and duct's tilting axis

L Lift

$l_{\text {duct }}$ Distance between duct's ac and tilting axis of the duct $\left(l_{\text {duct }}=c_{\text {duct }} / 4\right)$

$\mathrm{l}_{\mathrm{hs}} \quad$ Horizontal distance of horizontal stabilizer's ac to the $\mathrm{cg}$

m Mass

M Pitching moment

$\mathrm{M}_{\text {ram }}$ Pitching moment due to ram drag

$p \quad$ Pressure

P Power 


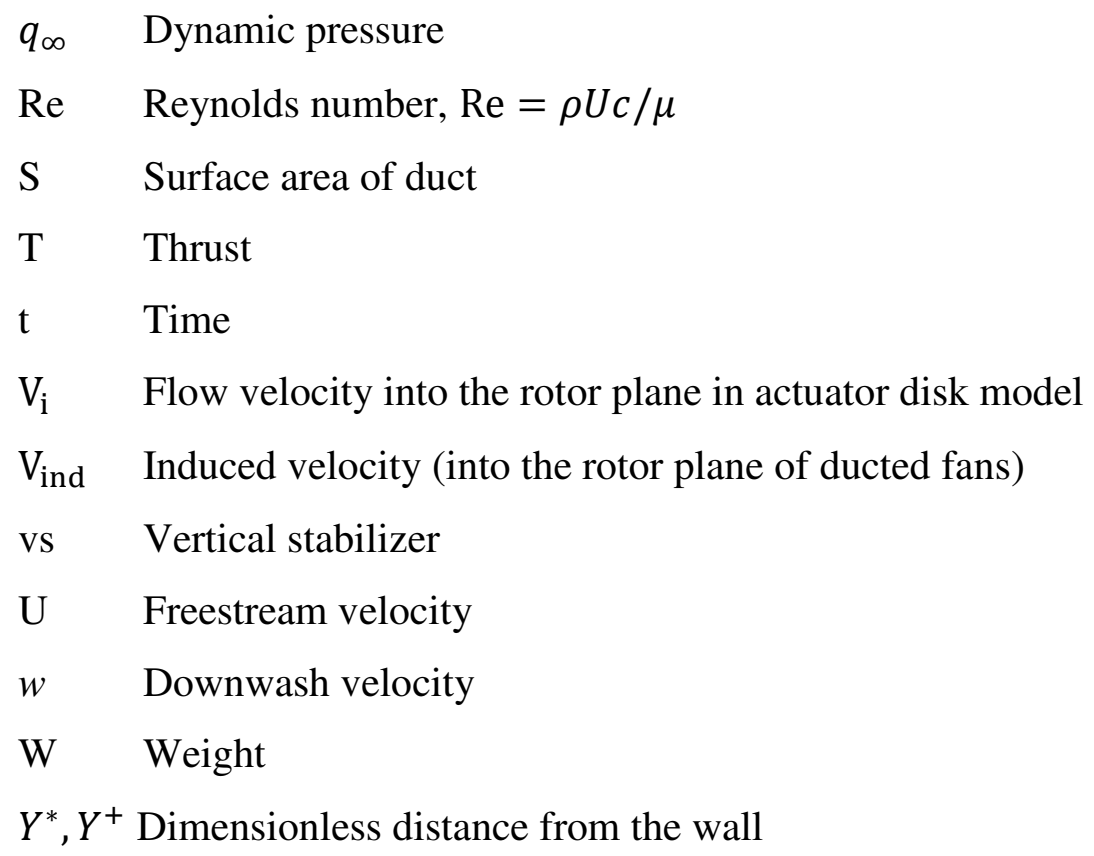

\section{Greek Letters}

$\alpha \quad$ Angle between main flow direction and thrust line of ducted fan

$\rho \quad$ Density of fluid

$\omega \quad$ Tilting rate $(\mathrm{deg} / \mathrm{sec})$

$\mu \quad$ Viscosity 


\section{INTRODUCTION AND MOTIVATION}

\subsection{Introduction}

Today, the fast growing applications of the unmanned aerial vehicles (UAVs) in different fields bring the need for the improvement of the UAVs' performance. Their potential for the low cost flights and simplicity of operation are strong reasons to dedicate more resources in this field. Although progress in propulsion systems, electronics, and composite materials plays a significant role in making UAVs smaller and more powerful, there are other areas to explore for improving the performance of UAVs. A typical UAV mission profile consists of reaching to a specific area in a short time, hovering while gathering information, and leaving that area as fast as possible. The typical mission profile becomes complicated when there is no specific airport for take-off and landing of that UAV and it has to fly a long distance for the mission. This complicated mission profile requires UAVs to be capable of performing take-off and landing in any arbitrary location, flying like an airplane and hovering like a helicopter.

The vertical take-off and landing (VTOL) UAVs can provide hovering same as small helicopters by using open rotors or propellers. However, this approach has several drawbacks. For example, open rotors/propellers cause tremendous amount of noise while rotor blades are subject to large tip-losses. Also, the low-height rotor installation can be dangerous for the ground crew. Such drawbacks in addition to the poor efficiency in forward flight make this configuration undesirable. Figure 1-1 shows the size of one recent helicopter base UAV. 

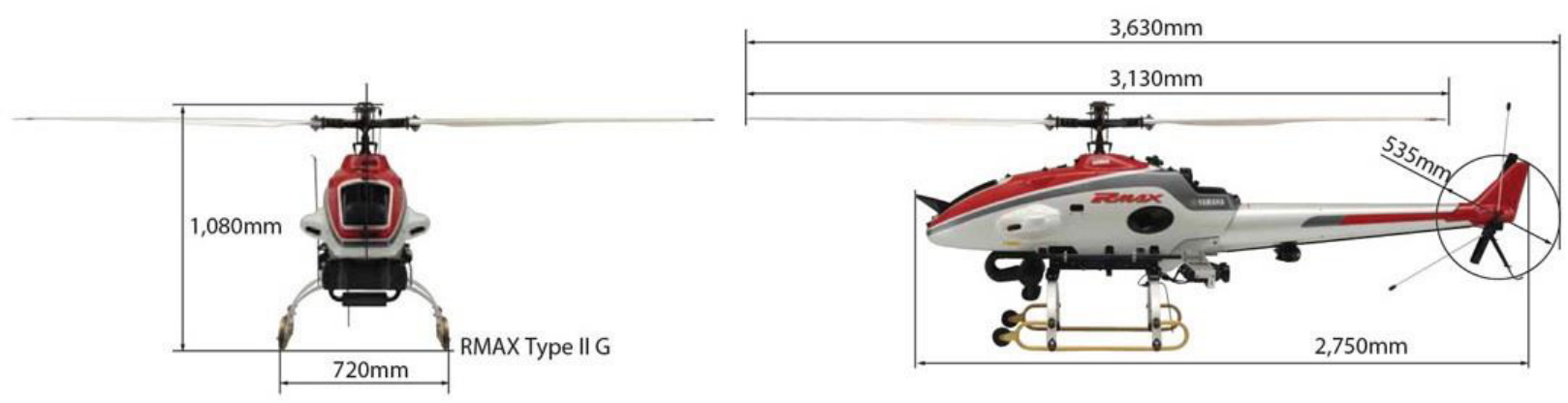

Figure 1-1: Yamaha R-Max helicopter (Public domain [1])

The ideal technical solution for this problem is using ducted fan instead of open rotors/propellers. Ducted fans provide a higher static thrust-to-power ratio for a given diameter than open propellers, due to the diffusion of the rotor jet stream behind the duct [2]. They are quieter and safer than open propellers as duct absorbs noise and keeps the rotating fan away from the ground crew.

One of the most common problems for both ducted fans and open propellers is flow separation during horizontal forward flight, which happens when some parts of relative inlet flow have steep rotation to enter the rotor plane. The flow separation is more extensive for open propeller as the inlet flow has a direct impact on the blade tips and outlet jet on the windward side. As simulated and compared in Figure 1-2, large parts of open rotor in the windward side experienced flow separation and this creates positive pitching moments.

Ducted fans can provide maximum convergence for the flow streamlines into the rotor plane because of inlet lip roundness. The tip-loss is less for ducted fans due to small gap between the blade tips and duct inner surface. There are several types of ducted fan UAVs and they are being used for different purposes. Figure 1-3 shows some of recent ducted fan UAVs. 


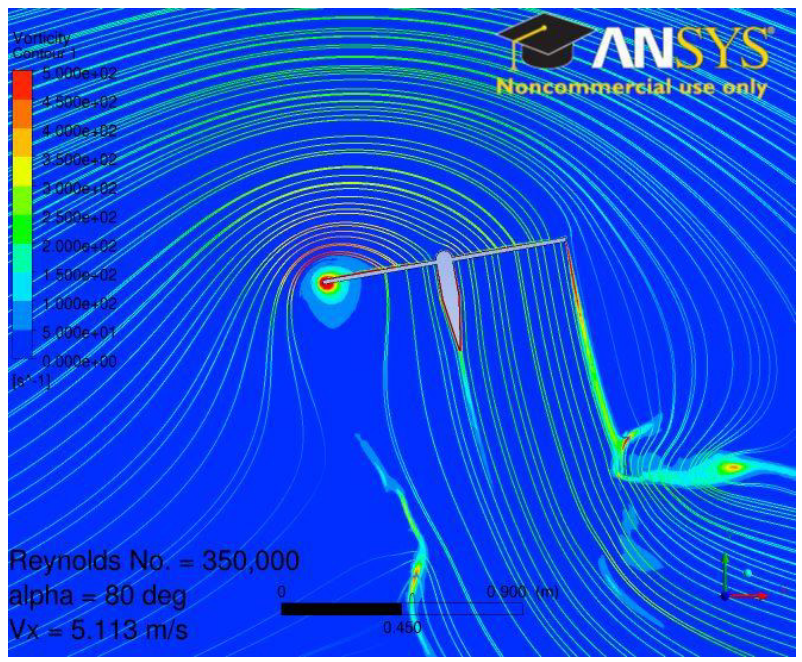

(a)

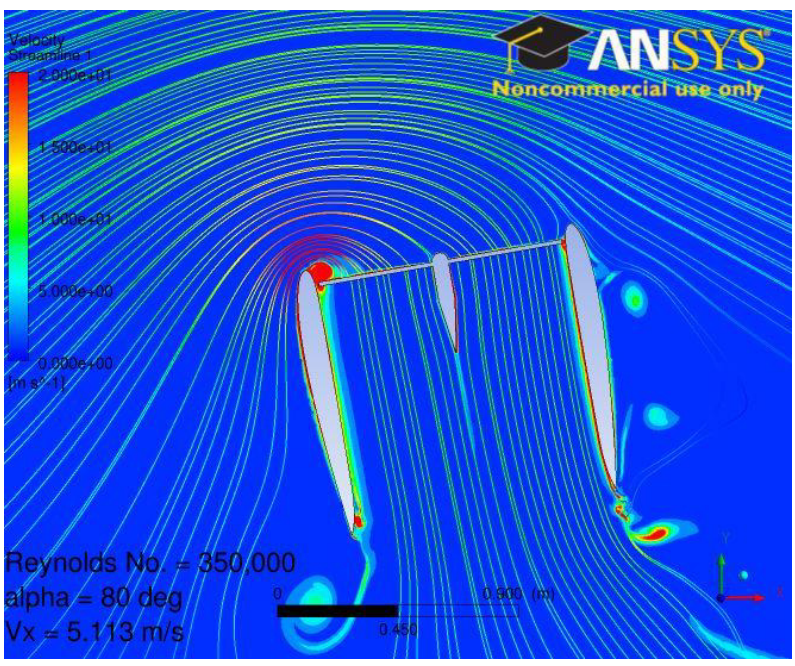

(b)

Figure 1-2: Comparison between streamlines into (a) an open propeller and (b) a ducted fan in a flow with $\mathrm{Re}=350,000, \mathrm{AoA}=80^{\circ}, \mathrm{V}_{\mathrm{x}}=5.113 \mathrm{~m} / \mathrm{s}$ and $\mathrm{V}_{\text {inlet }}=10.226 \mathrm{~m} / \mathrm{s}$

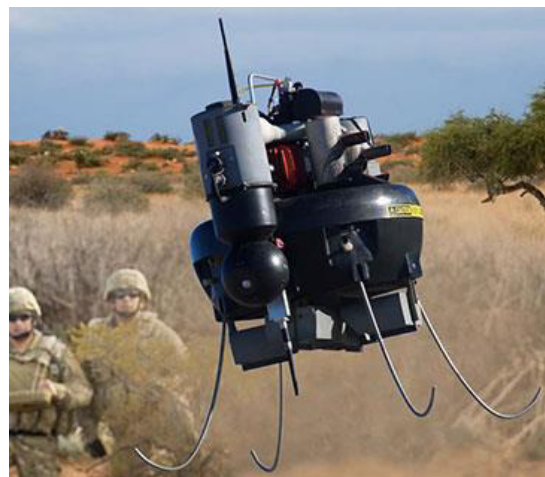

(a)

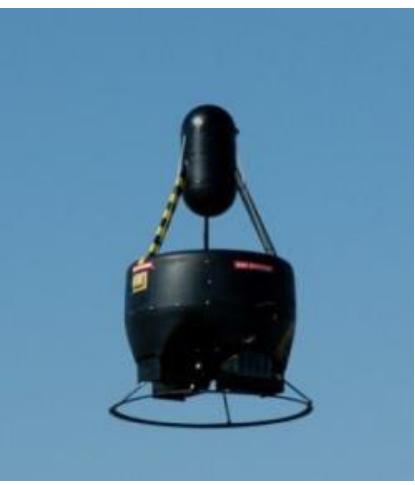

(b)

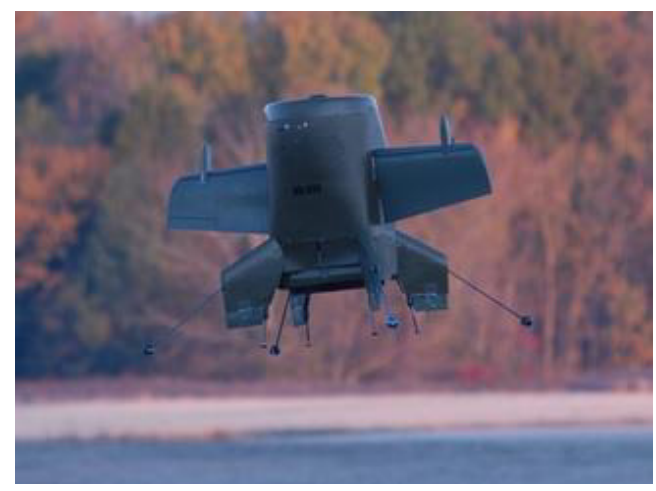

(c)

Figure 1-3: The most recent ducted fan UAVs: (a) Honeywell T-Hawk (Public domain [3]), (b) BAE Systems IAV2 (Public domain [4]), (c) Aurora Golden Eye-50 (Public domain [5])

Having the thrust line of ducted fan parallel to the flight path creates a uniform flow to the rotor disk and results in faster forward velocity for ducted fan UAV. Many UAVs are using only one ducted fan. Those single ducted fan UAVs without flying surfaces (as shown in Figure 1-3a and Figure 1-3b) have low speed forward flight in the range of 10 to $20 \mathrm{~m} / \mathrm{s}$. They are less efficient in horizontal flight due to the high angle of attack (AoA) between flight direction and thrust line, as generated ram drag creates a large nose-up pitching moment. The high AoA between flight direction and thrust line can cause a sequence of events such as: flow separation around the leading 
edge of the duct lip, distortion of the distribution of the velocity magnitude at the inlet, reduction of the breathing area at the fan rotor, and thrust reduction. Based on the research done by several researchers (e.g. [8]-[17]), there are few solutions to solve aforementioned weaknesses, but each solution has its specific disadvantages. The first method is improving the shape of duct lip to delay flow separation in both forward flight and hover. However, it is hard to find an optimum lip shape for both conditions. The second is adding some equipment to provide a uniform flow to the rotor plane, which results in increasing the weight and drag of the ducted fan. The third solution is increasing delivered power to the fan/propeller to reduce the effect of the leading side separation bubble, which results in consuming more energy for reattachment of the flow to the leading edge of the duct lip. The ideal solution is minimizing the AoA between the thrust line and flight path, which can provide uniform flow to the rotor disk, higher thrust, faster forward velocity, less drag, and longer range and endurance in horizontal flight. However, this would need wings and control surfaces to be added to the body of the UAV.

As shown in Figure 1-3c, adding flight surfaces can increase the UAVs speed in horizontal flight up to $45 \mathrm{~m} / \mathrm{s}$. This will increase the unwanted gyroscopic moment of rotating fan during conversion from cruise mode to hover, and will affect the stability of UAV, especially for those UAVs similar to Aurora Golden Eye-50 with a larger range of tilting angle. The other disadvantage of this configuration is the necessity of tilting the whole fuselage of the UAV in hover for surveillance and capturing images.

\subsection{Motivation}

The rotating fan has two major negative effects of "unwanted fuselage rotation and gyroscopic moment" during UAVs flights. All single ducted fan UAVs have a set of equipment such as "exit 
vane flaps" for redirecting the jet stream behind the duct to prevent fuselage rotation in hover due to the rotating fan. The rotating fan also creates unwanted gyroscopic moment during rapid maneuvers and conversion between hover and cruise mode. This moment has negative effects on the stability of single ducted fan UAVs and needs to be cancelled.

Assume $I_{R}$ as the rotor's moment of inertia about its spin axis, $\dot{\phi}$ as rotor rotational speed $(\mathrm{rad} / \mathrm{s})$ and $\dot{\theta}$ as tilting speed $(\mathrm{rad} / \mathrm{s})$ of ducted fan about y-axis, as shown in Figure 1-4. Then, the generated gyroscopic moment of $I_{R} \dot{\phi} \dot{\theta}$ about z-axis will be applied to the UAV fuselage during tilting about y-axis. This additional unwanted gyroscopic moment acts as a yaw moment on the fuselage of the UAV and has negative effects on the directional stability of the UAV during tilting.

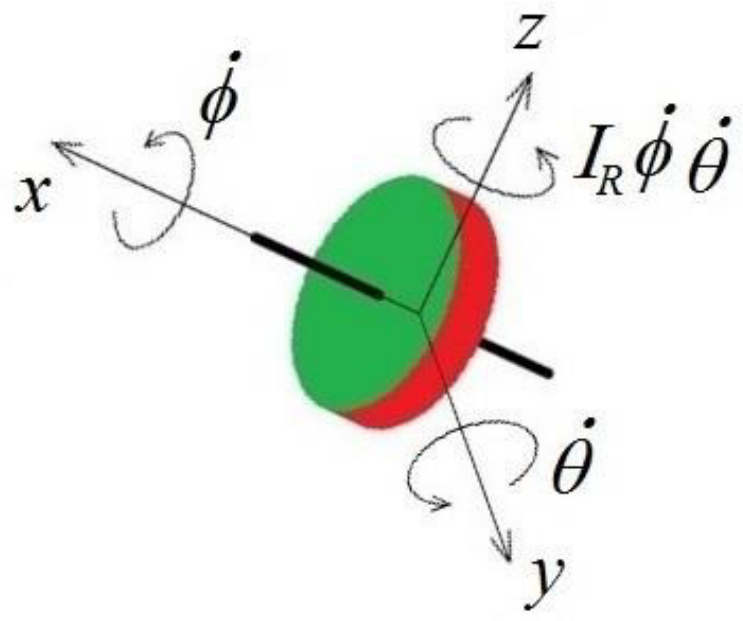

Figure 1-4: The generated gyroscopic moment of $I_{R} \dot{\phi} \dot{\theta}$ (about z-axis) on tilting ducted fan

One logical solution to the aforementioned issues is using the combination of fuselage and wing with two counter-rotating tilting ducted fans installed at the wing tips (Figure 1-5), which gives such UAVs the ability to take-off and land like a helicopter, as well as cruise like an airplane. This will increase the velocity, range and endurance of the flight, cancel the ram drag and its related pitching moment in cruise flight, prevent the fuselage rotation and cancel the gyroscopic moments 
during rapid maneuvers due to the rotating fans. One important advantage of the proposed VTOL UAV is minimizing the angle between flight path and thrust line. Moreover, tilting ducted fans installed on the wing tips of VTOL UAV provide thrust vectoring ability, which can be used for necessary maneuvers such as yaw in hover and roll and steady banked turn in cruise mode [6]. This configuration provides ideal image capturing for cameras attached to the fuselage in both cruise mode and hover.

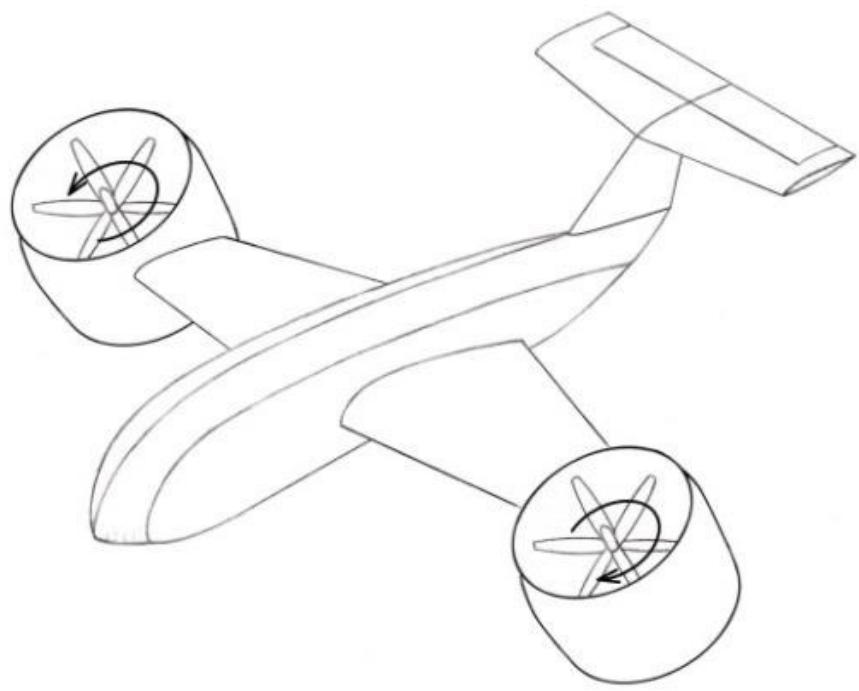

Figure 1-5: Schematic of the the proposed VTOL UAV with two tilting ducted fans installed on the wing tips

This research will discuss several main topics related to ducted fans in the next chapters as follows:

1. The aerodynamics of tilting ducted fans

2. Wind tunnel experiments on the symmetric and asymmetrical ducted fans and comparing the results with the CFD modeling

3. The applications of ducted fans in the VTOL UAVs and possibility of using asymmetrical ducted fans in the VTOL UAVs 
4. Calculating the induced velocity of the inlet flow to the rotor plane as it is one important parameter for computing aerodynamic forces and pressure distribution around the ducted fans

5. CFD simulation of aerodynamic forces and pitching moments on the tilting ducted fans in the transition conditions

6. Simulation of level flight for the proposed VTOL UAV in the transition from cruise mode to hover 


\section{BACKGROUND AND LITERATURE REVIEW}

\subsection{Background}

The research for development of vertical and/or short take-off and landing (V/STOL) aircraft started in 1950s. These aircraft can take-off and land vertically or from short runways. One important parameter for the V/STOL aircraft is ability for hover during their missions. A vertical take-off and landing (VTOL) airplane is a subset of the V/STOL aircraft, which does not need runways for take-off or landing. Hundreds of V/STOL aircraft were designed in the last 60 years but most of them were experiments or failed in the first flights and never became operational. Only few V/STOL aircraft went for mass production such as Harrier, Yak-38 Forger, V-22 Osprey and Lockheed Martin F-35B. Table 2.1 shows the important V/STOL aircraft with brief explanation about each one [7].

The idea of using tilting ducted fans attached to the wing tips is not new, but it seems still needs more improvement as there is no operational VTOL aircraft with tilting ducted fans attached to the wing tips yet. As mentioned before, ducted fans provide a higher static thrust-to-power ratio for a given diameter than open propellers due to the diffusion of the rotor jet stream behind the duct [2]. So, it is beneficial to use tilting ducted fans attached to the wing tips for a VTOL aircraft as it can provide thrust vectoring to control aircraft as well. This idea is more useful for designing an unmanned aerial vehicle (UAV) as it will be available for all surveillance missions very quickly.

The proposed VTOL UAV with two tilting ducted fans installed on the wing tips shown in Figure 1-5 has the ability to fly same as an airplane and also hover, take-off and land same as a helicopter from any arbitrary location. This research will discuss the aerodynamic behaviour of tilting ducted fans which can be used for this purpose.

Table 2.1: List of V/STOL aircraft from 1950s [7] 


\begin{tabular}{|c|c|}
\hline Date & \\
\hline Jan. 1954 & Bell 65 Experimental Tilt-jet VTOL \\
\hline 11 Aug. 1955 & Bell $X V$-3 Experimental Tilt-rotor VTOL \\
\hline 25 Feb. 1958 & Doak VZ-4 VTOL research convertiplane \\
\hline 26 May 1958 & Short SC.1 Experimental aircraft with \\
\hline 15 Aug. 1959 & Kamov Ka-22 Experimental transport aircraft with separate thrust and lift \\
\hline 24 Nov. 1959 & Hiller $\mathrm{X}$-18 Experimental prototype Tilt-wing \\
\hline 19 Nov. 1960 & $\begin{array}{l}\text { Hawker P.1127/Kestrel: } \text { Experimental V/STOL aircraft with four rotating } \\
\text { nozzles for vectored thrust of fan and jet exhaust. }\end{array}$ \\
\hline 1961 canceled & $\begin{array}{l}\text { Bell D-188A Experimental VTOL supersonic fighter (up to Mach 2) with } \\
\text { swivelling engines, was in mock-up stage }\end{array}$ \\
\hline 1961 canceled & Bell XF-109 Experimental Tilt-jet \\
\hline 7 Jul. 1962 & Lockheed $X V-4$ Hummingbird with separate thrust and lift \\
\hline 13 Oct. 1962 & Dassault Balzac V (Modified Mirage III) with separate thrust and lift \\
\hline 10 Apr. 1963 & $\begin{array}{l}E \boldsymbol{W} \boldsymbol{R} \boldsymbol{V} \boldsymbol{1 0 1} \mathrm{V} / \mathrm{STOL} \text { supersonic fighter ( up to Mach 2) with Tilt-jet, flown } \\
\text { to M1.04 but not operational }\end{array}$ \\
\hline Nov. 1963 & $\begin{array}{l}\text { Curtiss-Wright } X-19 \text { Experimental VTOL Transporter with four rotating } \\
\text { propellers (Tilt-Rotor) }\end{array}$ \\
\hline 25 May 1964 & Ryan $X V-5$. Fans in wings driven by engine exhaust gas. \\
\hline 29 Sep. 1964 & $\begin{array}{l}\text { LTV XC-142 Experimental VTOL transport with four-engine tilt-wing cross- } \\
\text { shafted turboprop }\end{array}$ \\
\hline 12 Feb. 1965 & $\begin{array}{l}\text { Dassault Mirage IIIV Delta wing Mach } 2 \text { fighter with lift engines, first VTOL } \\
\text { capable of supersonic and M2 flight (Mach } 2.03 \text { during tests) with separate } \\
\text { thrust and lift }\end{array}$ \\
\hline 12 Feb. 1965 & Hawker Siddeley P.1154 M1.7 Supersonic Harrier. It was not completed \\
\hline 7 May 1965 & $\begin{array}{l}\text { Canadair CL-84 Dynavert, Experimental VSTOL Tilt-wing with two } \\
\text { turboprop in RCAF service from } 1960\end{array}$ \\
\hline 17 Mar. 1966 & $\begin{array}{l}\text { Bell } \boldsymbol{X} \text {-22 Small V/STOL transport prototype with rotating ducted propellers. } \\
\text { Slightly smaller than V-22 Osprey. }\end{array}$ \\
\hline
\end{tabular}




\begin{tabular}{|c|c|}
\hline 10 Feb. 1967 & $\begin{array}{l}\text { Dornier Do } 31 \text { VTOL jet transport with podded vector nozzles and lift engines } \\
\text { with separate thrust and lift }\end{array}$ \\
\hline 28 Dec. 1967 & $\begin{array}{l}\text { Harrier V/STOL strike aircraft with four rotating nozzles for vectored thrust } \\
\text { of fan and jet exhaust. }\end{array}$ \\
\hline 1971 & Yakovlev Yak-38 VTOL Fighter aircraft with separate thrust and lift \\
\hline 20 Sep. 1971 & $\begin{array}{l}\text { VFW VAK 191B VTOL strike aircraft similar to Harrier, smaller wings and } \\
\text { lift engines with separate thrust and lift. }\end{array}$ \\
\hline 3 May 1977 & Bell $\boldsymbol{X V} \mathbf{- 1 5}$ Experimental VTOL aircraft with 2 Tilt-rotors \\
\hline 1981 cancelled & $\begin{array}{l}\text { Rockwell } \boldsymbol{X F} \text { V-12 Supersonic VTOL Fighter. Built with complex "window } \\
\text { blind" wings but could not lift its own weight. }\end{array}$ \\
\hline 9 Mar. 1987 & $\begin{array}{l}\text { Yakovlev Yak-141 Supersonic VTOL Fighter aircraft with separate thrust and } \\
\text { lift. Lift engine plus swivel tailpipe, somewhat like F-35. }\end{array}$ \\
\hline 24 Oct. 2000 & $\begin{array}{l}\text { Lockheed Martin } X-35 B \text { / F-35B uses a vectored-thrust tailpipe (the Pratt \& } \\
\text { Whitney F135) plus a shaft-driven lifting fan. First aircraft capable of } \\
\text { demonstrating transition from short take-off to supersonic flight to vertical } \\
\text { landing on the same sortie. }\end{array}$ \\
\hline 6 Mar. 2003 & Bell 609 VTOL corporate transport with 2 Tilt-rotors \\
\hline 13 Jun. 2007 & $\begin{array}{l}\text { Bell-Boeing } \mathbf{V - 2 2} \text { Osprey V/STOL military transport aircraft (scale up of XV- } \\
\text { 15) with } 2 \text { Tilt-rotors }\end{array}$ \\
\hline Jun. 2011 & $\begin{array}{l}\text { Agusta Westland Project Zero Experimental tiltrotor VTOL aircraft. } \\
\text { Technology demonstrator of Fan-in-Wing }\end{array}$ \\
\hline
\end{tabular}

\subsection{Literature review}

The study of aerodynamic behaviour of tilting ducted fans in transition between hover and cruise mode is essential to understand and predict the performance of VTOL UAVs with tilting ducted fans installed on their wing tips.

Most of the research were done in the fields of: modeling, control, flight test, tip clearance, duct lip shaping, various control devices, aerodynamic, and performance of ducted fans in hover and 
crosswind conditions in the last six decades. The following are important research outcomes published in the fields of aerodynamic and performance of ducted fans, which could be useful for explaining and verifying the results in this research.

Extensive research has been done on the flow field properties around ducted fans in different conditions. Martin and Tung [8] measured aerodynamic loads on a ducted fan UAV with a 10-inch diameter fan rotor in different AoA in hover and different crosswind velocities. They found that at low RPM, the viscous losses of the duct internal flow counteracted the duct thrust, which is an important result for designing micro UAVs. They also showed that by increasing the rotor tip gap, the duct thrust decreased dramatically. The other important result was that by decreasing duct leading edge radius, the ratio of duct thrust to isolated propeller thrust was decreased, but the stability for hovering in a crosswind was improved.

Wind tunnel tests and numerical investigations on the performance of a 12 -inch ducted fan in crosswind were reported by Fleming et al. [9]. They studied several important design considerations such as stabilizing or righting torque on the duct lip due to the presence of a crosswind, and the positioning of the control vanes in consideration of duct jet bending and maintaining a natural tilting effect in gusts.

The leading edge geometry of ducts, which is an important parameter in offsetting the effects of adverse aerodynamic characteristics, was examined in experiments by Graf et al. [10] to improve ducted fan performance in forward flight. They found that the performance of a lip shape with a small leading edge radius was the best in forward flight and crosswind conditions, which was due to the reduction in pitching moment. Whereas, a lip shape with a large leading edge radius performed best in static condition that resulted in thrust improvement. 
The flow separation around the leading edge of the duct lip is an important phenomenon. The separation occurs when the relative inlet flow has a sharp turn in entering to its inlet plane during horizontal flight. Akturk et al. [11] used a planar particle image velocimeter (PIV) system to investigate the near duct aerodynamic performance in hover and forward flight. Their experiments showed that the flow separation from the leading side at the inlet duct lip distorted the distribution of the velocity magnitude at the inlet and resulted in the reduction of the breathing area at the fan rotor. The inlet flow distortion caused a drop in thrust force generated by the ducted fan system. Finally, the inlet flow distortion generated excessive moment imbalance and non-uniformities in the exit jet of their ducted fan model in the forward flight condition. They reported that an increase in the rotational speed of the fan (from $9000 \mathrm{rpm}$ to $15000 \mathrm{rpm}$ in their experiments) could reduce the effect of the leading side separation bubble.

Camci and Akturk proposed the "Double Ducted Fan", by adding a secondary stationary duct system to control "inlet lip separation" related momentum insufficiency at the inlet of the fan rotor occurring at elevated forward flight velocities [12]. Although they reported an improvement in the thrust due to reduction in the flow separation at the leading edge of the duct lip, there is a wide area with the separated flow at the leeward side of the secondary stationary duct, which increases the total drag and pitching moment of the double ducted fan.

Tilting ducted fans mounted on the wing tips were used in Doak VZ-4-DA and Bell Aerosystems Company X-22A airplanes. Both projects were cancelled by the US Army in 1963 and 1967 due to weaknesses in their performance. The aerodynamic characteristics of the sevenfoot-diameter ducted propeller used on the Bell X-22A for variations of power, free-stream velocity, blade angle, and duct AoA were investigated and reported by Mort and Games [13]. They found the stall conditions as a function of duct AoA for both the upstream and downstream duct 
lips. An important result was that the complete flow separation happens with high AoA and low power.

Yaggy and Mort $[14,15]$ also tested the performance of a four-foot-diameter ducted fan used on Doak VZ-4-DA in hover and forward flight conditions. They found that the flow separation at the windward side of the duct lip was the reason for the rapid changes in the pitching moment and increment in the required power. One important result from their report was that the ducted fans installed on the wing tips needed less power compared to the free flying ducted fans.

Moving ducted fans in freestream can create well-known aerodynamic forces and moments such as lift, drag and pitching moment. Ram drag is another important force which is generated due to turning of the oncoming flow from the freestream direction to the axial direction of the ducted fan. As the centre of pressure for redirected flow is above the duct lip, this force can generate a large nose-up pitching moment about the centre of gravity of ducted fans. Ram drag can be larger than profile drag of the ducted fan's fuselage as it is proportional to the mass flow rate to the rotor plane and crosswind velocity. The open rotors or the shrouded rotor configurations with short duct chord lengths (or a large duct aspect ratio, $A R=d / c$ ) generate less ram drag related to ducted fans [9].

The ducts' inlet lip radius and configuration was studied theoretically and experimentally by Weir [16]. Measuring the aerodynamic forces and moments for different inlet lip configurations showed that by increasing the inlet lip radius the lift force of ducted fan was increasing slightly. Also, adding a diffuser to the system could increase the lift. However, the pitching moment was increased due to these two improvements.

The application of thrust vectoring for directional control of the single ducted fan UAV was studied by many researchers. The wind tunnel tests performed by Abrego and Bulaga showed the 
importance of exit vane flap deflection and flap chord length effects on providing side force [17]. Also, they showed that duct thrust coefficient was not affected by extending the duct chord length. The proposed VTOL UAV in this research can have best performance as tilting ducted fans installed at its wing tips can provide an appropriate angle between flight path and thrust line during cruise, maneuvering, and transition between cruise and hover. Tilting ducted fans can minimize flow separation around duct lips, which results in producing more thrust and saving more energy. The proposed VTOL UAV does not need any complicated control system such as control vane [9] or exit vane flaps [17] as a complete set of control systems (rudder and elevator in the tail) are being used for directional control. Thrust vectoring can have wide usage in the proposed model for directional control and maneuvering. It can be used for yaw in hover and roll and steady banked turn in cruise mode.

\subsection{Problem statement}

The performance of a ducted fan can be affected by various parameters. One important parameter is the shape of the duct's outer body, which has significant effects on the external flow field properties in cruise mode. All studied models in the previous research (e.g. [8]-[17]) were symmetrical ducted fans, and to the best of my knowledge, there was no specific published study for using an asymmetrical shape for external body of the ducted fans. Then, it is necessary to compare advantages and disadvantages of symmetrical and asymmetrical ducted fans.

The first objective of this research is to investigate the possibility of using an asymmetrical shape for the external body of the ducted fans to generate additional lift for the proposed VTOL UAV. This will help to design a smaller wing and reducing wing related drag to save more energy and increase the range and endurance of the flight. The second objective is to combine the actuator 
disk model [18] with the assumption of constant power used by the propeller to introduce an alternative method for predicting the velocity of the inlet flow to the rotor plane. This is necessary for computing thrust, ram drag, and aerodynamic forces and pitching moments during CFD simulations. The third objective is using CFD simulation for predicting aerodynamic forces and pitching moment on the tilting ducted fans in the transition conditions for different tilting rates. The fourth objective is using the aerodynamic coefficients of the tilting ducted fans to predict and compare the level flight conditions of the proposed VTOL UAV during transition between cruise mode and hover.

This research presents the wind tunnel experimental outcomes in addition to the CFD simulations numerical results, employed for predicting aerodynamic forces and pitching moments acting on the symmetric and asymmetrical ducted fans. The proposed asymmetrical model can be used as the external body of a ducted fan to provide additional lift in the cruise mode. 


\section{APPROACH TO CFD MODELING}

\section{1 "Momentum Theory" and "Actuator Disk Model"}

Momentum theory applies to propulsive systems where the flowing gas is mainly atmospheric air [19]. The propulsive system increases the energy of the outgoing air. The increment in the energy of the outgoing air is used to increase the rearwards momentum of the air to produce thrust of the propeller or engine. This theory is based on the concept of the ideal actuator disk. This is an infinitely thin disk of area $A$ which gives pressure energy to air passing through the disk without any resistance. The assumptions for an ideal actuator disk are as follows:

- The energy is being added uniformly over the whole area of the disk

- The velocity of the air through the disk is constant through the whole area

- All energy supplied to the disk is transferred to the air

- The flow through the rotor is one-dimensional

- The flow is quasi-steady, incompressible, and inviscid

Consider the system shown in Figure 3-1. The actuator disk is at rest in a fluid which far away ahead of the disk is quiescent (i.e., $U \approx 0$ ) and has a pressure of $p_{o}$. The outer curved lines show the streamlines separating the fluid which passes through the disk from that which does not.

The velocity of fluid is increased to $V_{i}$ just before entering the actuator disk and its pressure drops to $p_{1}$. The fluid pressure is increased to $p_{2}$ after exiting from the actuator disc, but due to assuming constant density of the flow before and after the disk, the flow velocity does not have a sudden change. The air expands and accelerates after exiting the disk until its pressure returns to $p_{o}$ and its speed is $w$. The flow behind the disk which is bounded between streamlines is known as the slipstream. 


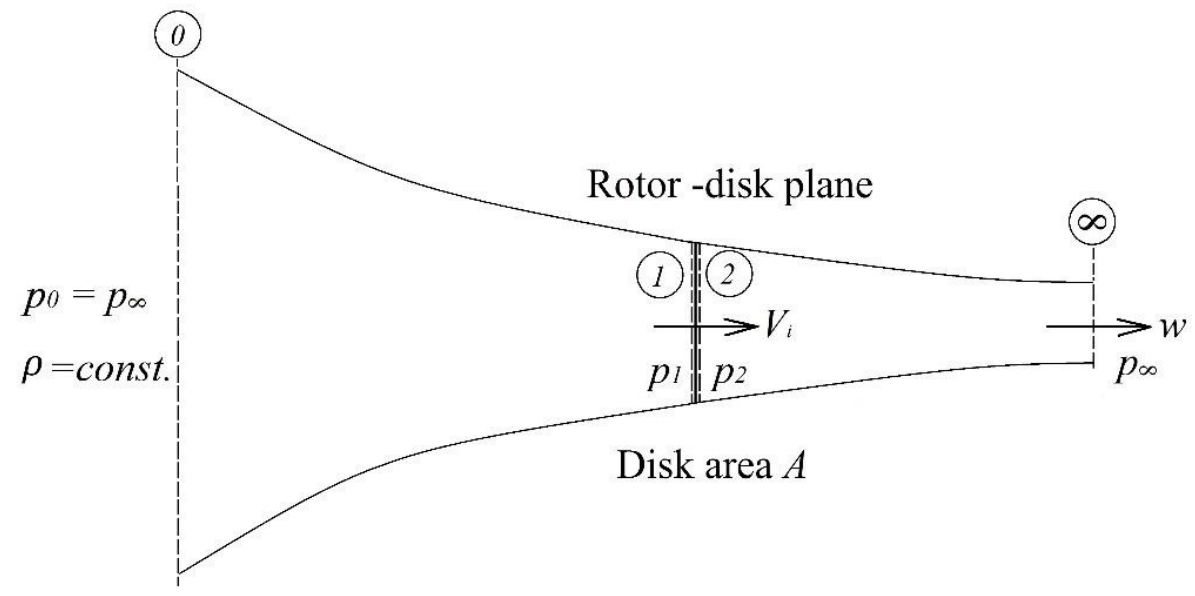

Figure 3-1: The ideal actuator disk and flow in the slipstream

The assumption of one-dimensional flow behind the disk helps to find the mass flow rate [18]:

$\dot{m}=\iint_{2} \rho \vec{V} \cdot d \vec{S}=\iint_{\infty} \rho \vec{V} \cdot d \vec{S}$

or: $\dot{m}=\rho A V_{i}=\rho A_{\infty} W$

Using Newton's second law gives the disk thrust, $T$, as the net time rate-of-change of fluid momentum out of control volume as follows:

$T=\iint_{\infty} \rho(\vec{V} \cdot d \vec{S}) \vec{V}-\iint_{0} \rho(\vec{V} \cdot d \vec{S}) \vec{V}$

The velocity of upstream fluid (far from the disk) is zero, then thrust can be found as:

$T=\dot{m} w$

Using the principle of conservation of energy, the work done on the disk should be equal to the gain in energy of the fluid per unit time. Thus, the work done per unit time, or the consumed power by the actuator disk, is $T V_{i}$ and can be written as:

$T V_{i}=\iint_{\infty} \frac{1}{2} \rho(\vec{V} \cdot d \vec{S}) \vec{V}^{2}-\iint_{0} \frac{1}{2} \rho(\vec{V} \cdot d \vec{S}) \vec{V}^{2}$ 
The second term on the right-hand side of the above equation is zero as the fluid is at the rest far ahead of the disk. Thus:

$T V_{i}=\frac{1}{2} \dot{m} w^{2}$

Combining equations (3.2) and (3.3) results in:

$V_{i}=\frac{1}{2} w$

The equation (3.4) explains a simple relationship between induced velocity $V_{i}$ and downwash velocity $w$, which is applicable for an ideal open rotor. Equation (3.2) can be written in the form of:

$T=\dot{m} w=\dot{m}\left(2 V_{i}\right)=2\left(\rho A V_{i}\right) V_{i}=2 \rho A V_{i}^{2}$

Solving for $V_{i}$ results in:

$V_{i}=\sqrt{T /(2 \rho A)}=\sqrt{\left(\frac{T}{A}\right) \frac{1}{2 \rho}}$

$T / A$ is known as disk loading and it is an important parameter for open rotor analysis. The power to the ideal open rotor can be express as:

$P=T V_{i}=T \sqrt{T /(2 \rho A)}=T^{3 / 2} / \sqrt{2 \rho A}$

or in the form of:

$P=T V_{i}=2 \dot{m} V_{i}^{2}=2\left(\rho A V_{i}\right) V_{i}^{2}=2 \rho A V_{i}^{3}$

which is ideal power, as the contribution of viscous effects have been ignored.

Momentum theory can be used to analyse the thrust and power of the ducted fan in this research as well. Figure 3-2 shows ducted fan model of this research. The definitions of parameters and boundaries are same as those in Figure 3-1. Ducted fans are designed to have specific ratio between the area of slipstream flow at the outlet and fan's area. This could be shown as $A_{\infty}=a_{w} A$ by introducing $a_{w}$ as wake contraction parameter. 


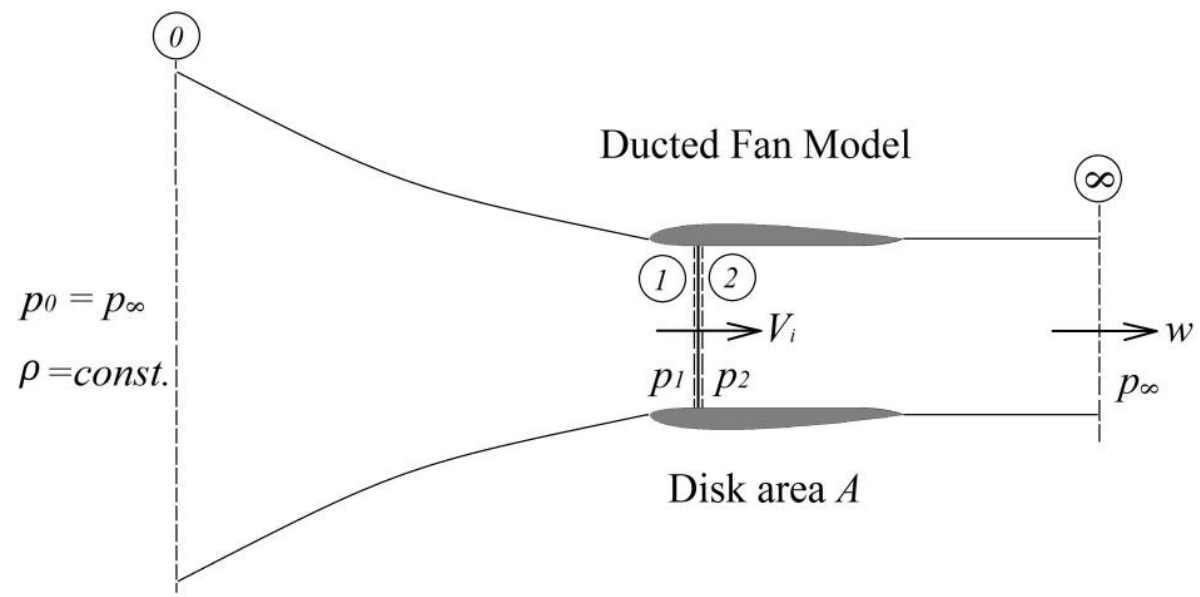

Figure 3-2: Ducted fan and flow model for using momentum theory

Knowing $A$ as fan area and using the principle of conservation of mass, then the mass flow rate can be written as:

$\dot{m}=\rho A V_{i}=\rho A_{\infty} w=\rho\left(a_{w} A\right) w$

which results in:

$w=V_{i} / a_{w}$

Total thrust on the duct and fan can be found by using conservation of momentum as follows:

$T=T_{\text {duct }}+T_{\text {fan }}=\dot{m} w=\rho A V_{i}^{2} / a_{w}$

or: $V_{i}=\sqrt{a_{w} T /(\rho A)}$

Bernoulli's equation can be used to calculate the thrust of the fan as following:

between station 0 and 1: $\quad p_{0}=p_{1}+\frac{1}{2} \rho V_{i}^{2}$

between station 2 and $\infty: \quad p_{2}+\frac{1}{2} \rho V_{i}^{2}=p_{0}+\frac{1}{2} \rho w^{2}$

and then:

$T_{\text {fan }}=\left(p_{2-} p_{1}\right) A=\frac{1}{2} \rho w^{2} A$

Combining equations (3.11) and (3.15) gives: 


$$
\frac{T_{f a n}}{T}=\frac{\frac{1}{2} \rho A w^{2}}{\rho A V_{i} w}=\frac{w}{2 V_{i}}=\frac{1}{2 a_{w}}
$$

The induced power to the fan can be found by using equations (3.12) and (3.16):

$$
\left(P_{i}\right)_{\text {fan }}=T_{\text {fan }} V_{i}=\left(\frac{T}{2 a_{w}}\right) \sqrt{\frac{a_{w} T}{\rho A}}=\frac{T^{3 / 2}}{\sqrt{4 a_{w} \rho A}}
$$

Comparing with the induced power to the open rotor which is given in equation (3.7):

$\frac{\left(P_{i}\right)_{f a n}}{\left(P_{i}\right)_{O R}}=\frac{1}{\sqrt{2 a_{w}}}$

where $\left(P_{i}\right)_{O R}$ refers to the induced power of the ideal open rotor. Equation (3.18) shows if $a_{w}=1$ (which is close to $a_{w}$ in this research), then the ducted fan will require only $71 \%$ of the power required by the open rotor to generate the same thrust.

\subsection{Duct models}

To study the effects of external shape on the aerodynamic coefficients, two ducted fans with different geometry for external bodies (one symmetrical and one asymmetrical) were simulated in this research. The external body of the symmetrical model is created by revolving the upper side profile of NACA 0012 airfoil, as shown in Figure 3-3.

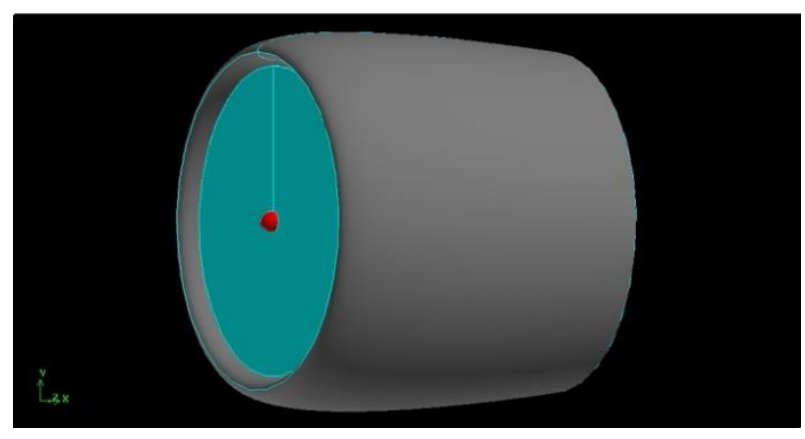

(a)

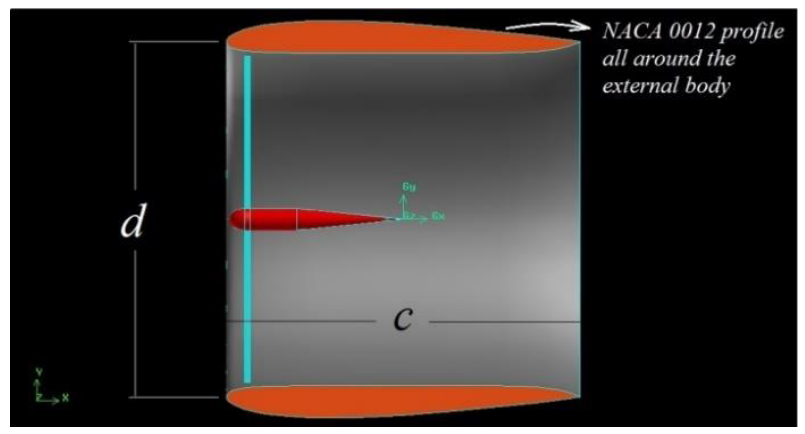

(b)

Figure 3-3: Symmetrical ducted fan with NACA0012 profile for external body: (a) 3D view, (b) Cross-section in xy plane 
The external body of the asymmetrical ducted fan consists of different profiles of high lift FX 63-137 airfoil, as shown in Figure 3-4. This airfoil is intended for human-powered aircraft in the wide range of Reynolds No. $\left(1 \times 10^{5} \leq R e \leq 1 \times 10^{6}\right)$ [20]. The profile of the upper side of the FX 63-137 airfoil is applied for $120^{\circ}$ on the upper side of the asymmetrical duct. Also, the profile of the lower side of the FX 63-137 airfoil is applied for $120^{\circ}$ on the lower side of the asymmetrical duct. There are two similar smooth transitional regions on the left and right sides of the duct that connect the lower part to the upper part.

The internal bodies for both symmetrical and asymmetrical ducted fans are similar, as shown in Figure 3-3b and Figure 3-4d. To reduce the number of parameters which have effects on this research, the mean diameters at the duct inlet/outlet are set equal to the duct chord length $(d=c)$ for both symmetrical and asymmetrical ducted fans, as shown in Figure 3-3b and Figure 3-4d.

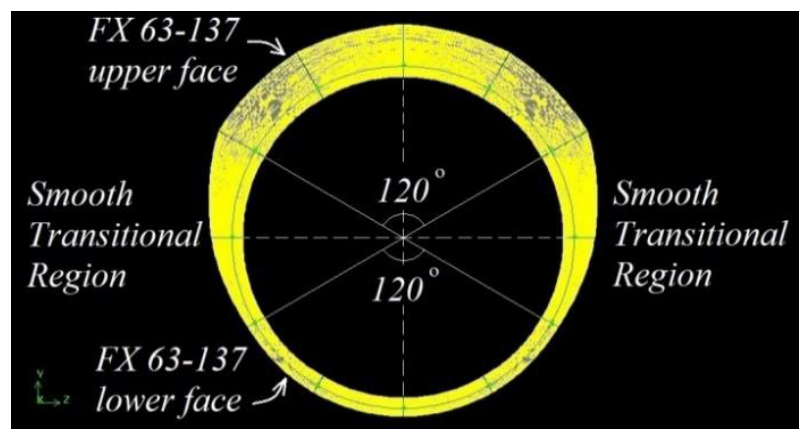

(a)

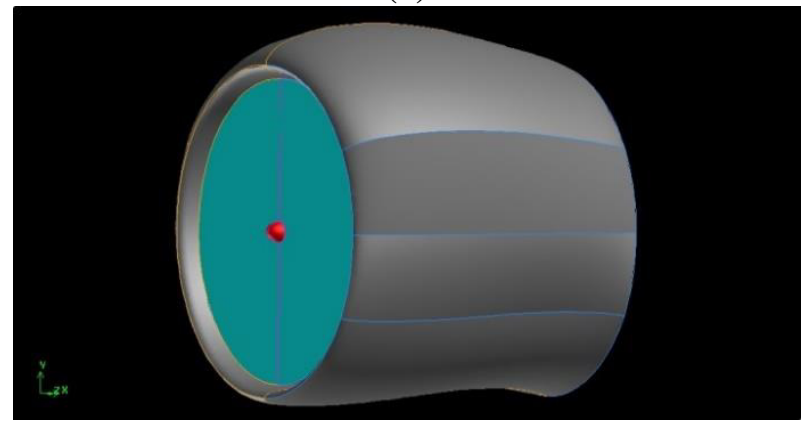

(c)

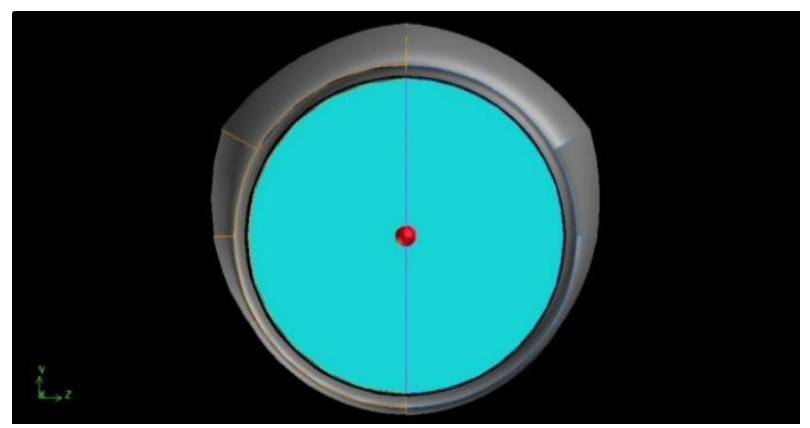

(b)

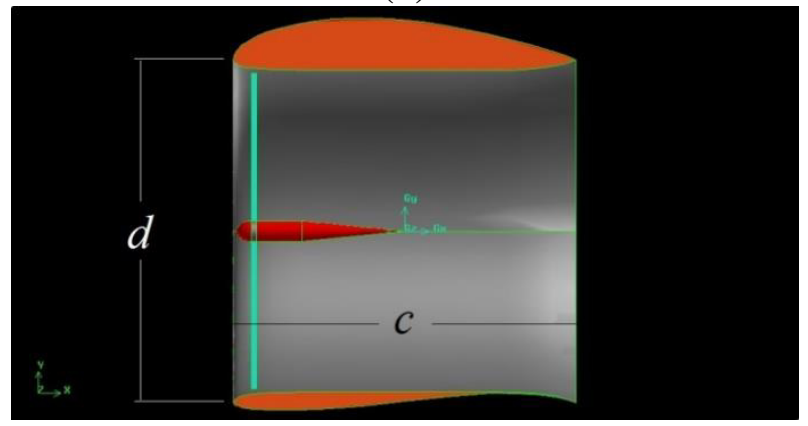

(d)

Figure 3-4: (a) Locations of different profiles of the high lift FX63-137 airfoil on the different sides of the external body of asymmetrical ducted fan, (b) Front view, (c) 3D view, and (d) Cross-section in xy plane 


\subsection{Governing equation, CFD code, mesh generation and computational domain}

The flow field of a 3D duct in an incompressible regime is governed by the Navier-Stokes equations in $x, y$, and $z$ directions as following:

Continuity: $\quad \nabla \cdot \vec{U}=0$

Momentum: $\quad \rho \frac{\partial \vec{U}}{\partial t}+\rho(\vec{U} \cdot \nabla) \vec{U}=-\nabla p+\mu \nabla^{2} \vec{U}$

where $\vec{U}=U(x, y, z, t)$ is the velocity vector, $t$ represents the physical time, $p=p(x, y, z)$ indicates the pressure, $\rho$ represents the density, and $\mu$ stands for viscosity.

ANSYS-Fluent is a CFD software package used to simulate fluid flow problems. It uses the finite-volume method to solve the governing equations [21]. The grid generation is done using GAMBIT software.

The selected models can tilt about the $z$-axis and as a result the $x y$ plane is the plane of symmetry for simulations. As shown in Figure 3-5a, the selected domain for solving Navier-Stokes equations is a sphere. The ducted fan is in the centre of spherical domain with a radius of 10 characteristic duct chord lengths.

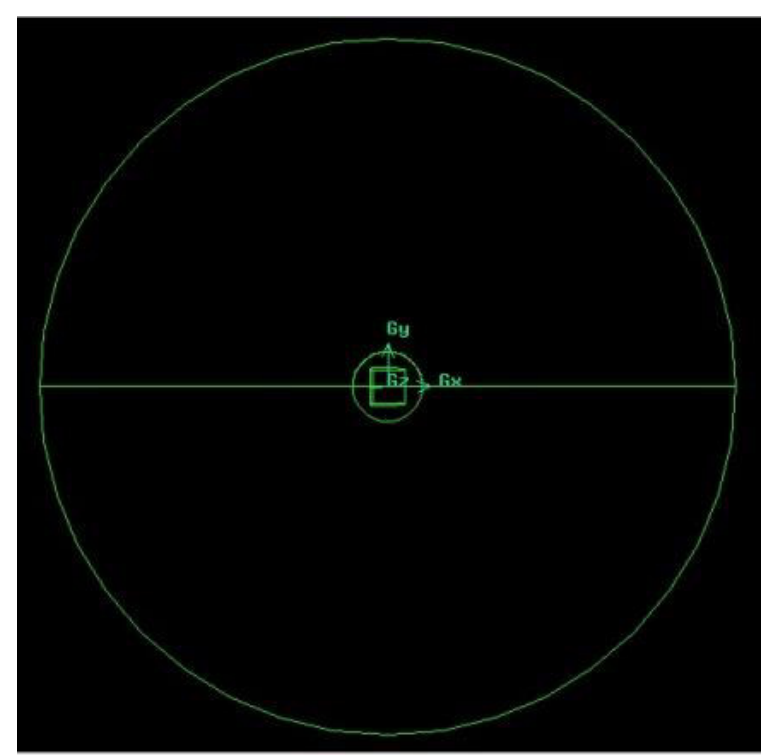

(a)

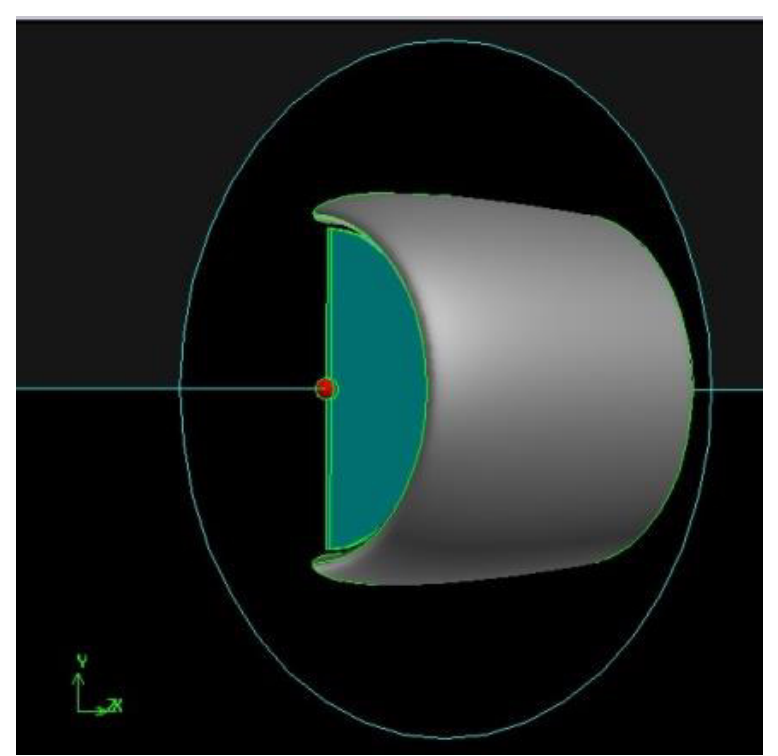

(b)

Figure 3-5: (a) Computational domain and (b) interior border around the duct 
The 3D discretized Navier-Stokes equations are used for incompressible flow by ANSYSFluent. The interior border around the ducted fan consists of a sphere of radius 1 characteristic duct chord length, as shown in Figure 3-5b.

The volumetric cells are tetrahedrons with triangular faces for the spherical boundaries and all faces in the domain are divided into triangular cells, as shown in Figure 3-6. The grids are very fine around the duct and become coarse as they go farther from the ducted fan, as shown in Figure 3-6.

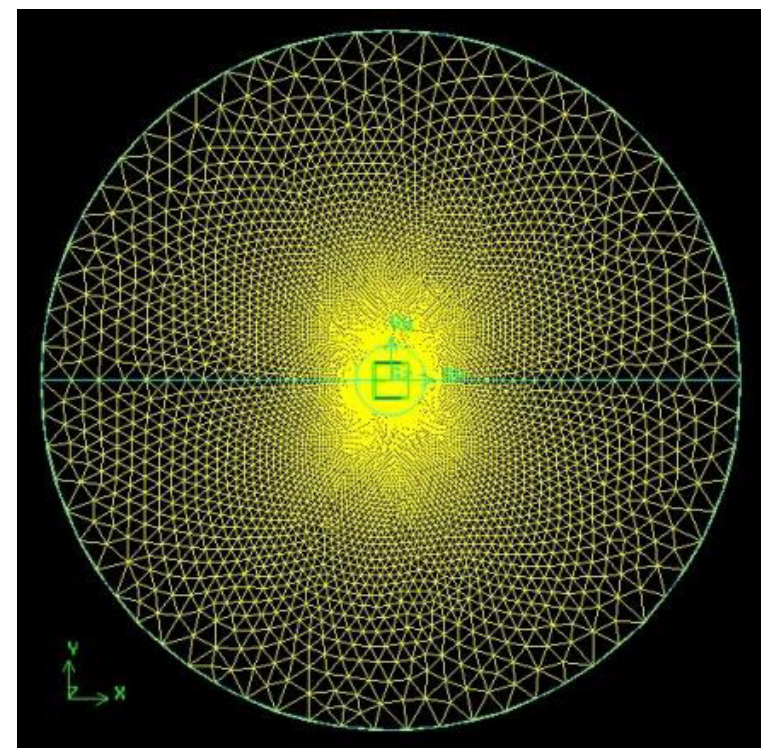

(a)

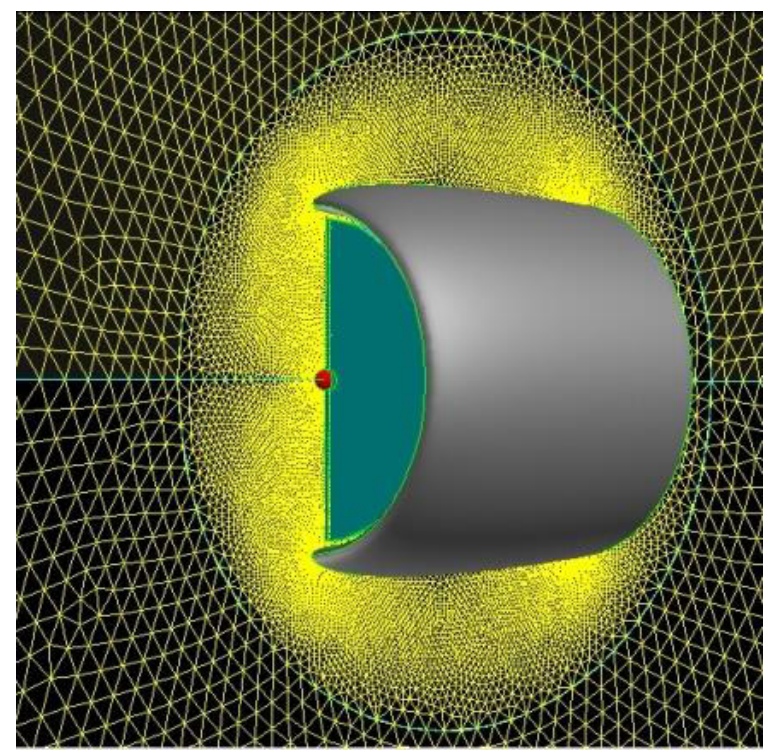

(b)

Figure 3-6: Comparing the size of cells for (a) whole domain, and (b) around the ducted fan

\subsection{Defining aerodynamic forces and pitching moment acting on the models}

The aerodynamic forces and pitching moments acting on the models can be described as follows:

1- For the ducts without fans, as shown in Figure 3-7, the components of the aerodynamic forces in the $x, y$, and $z$-directions can be described in the form of drag and lift forces by using the following equations: 


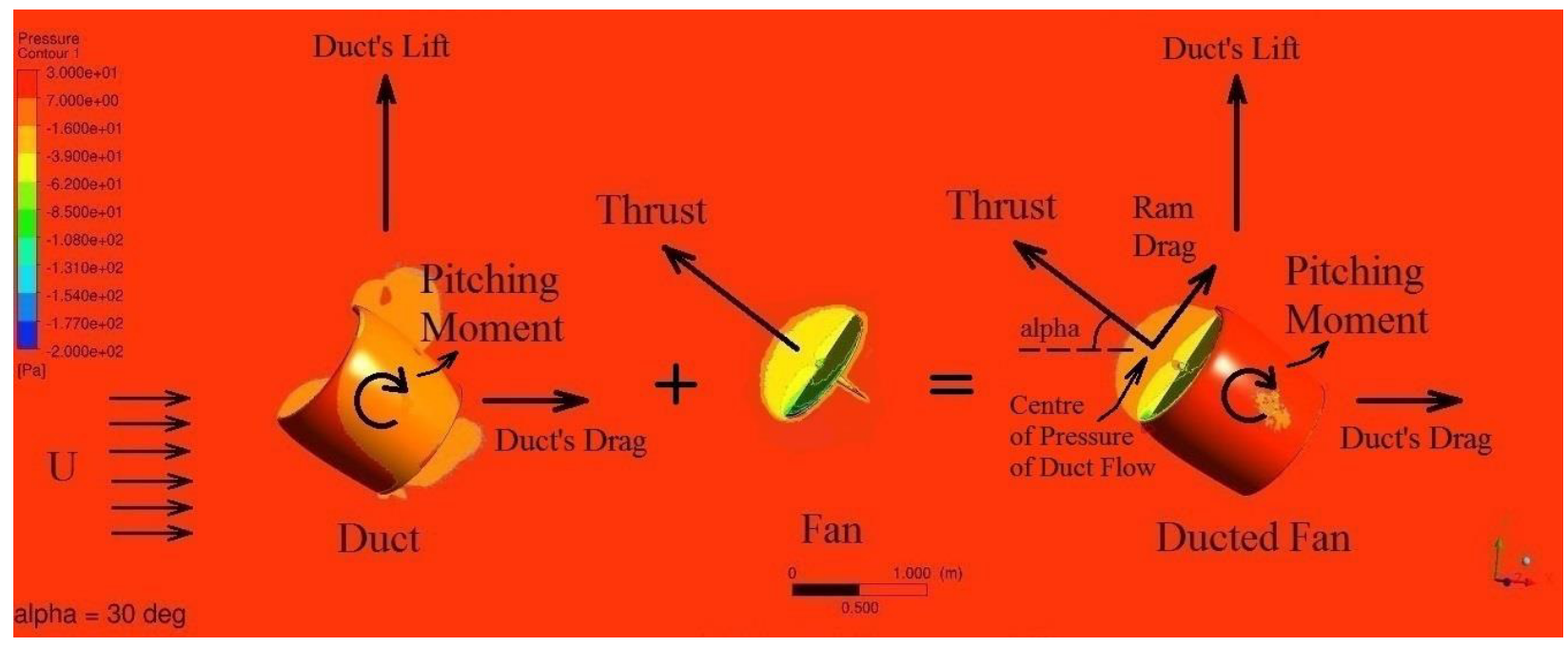

Figure 3-7: Contour of pressure distribution and the resultant aerodynamic forces and pitching moment acting on the external body of a ducted fan

$F_{x}$ is the $\mathrm{x}$ component of the resultant force or Drag $=\frac{1}{2} \rho U^{2} S C_{D}=q_{\infty} S C_{D}$

$F_{y}$ is the y component of the resultant force or Lift $=\frac{1}{2} \rho U^{2} S C_{L}=q_{\infty} S C_{L}$

$F_{z}=0$ due to the symmetry of the ducts' shape relative to the $x y$ plane. The components of aerodynamic moment in $x$ and $y$ directions are both zero $M_{x}=M_{y}=0$, as the $x y$ plane is the plane of symmetry for the models. The $z$ component of the moment (pitching moment) is given by:

$M_{Z}=\frac{1}{2} \rho U^{2} S c C_{M}=q_{\infty} S c C_{M}$

If the tilting axis of the duct does not pass through the aerodynamic centre or centre of gravity of the duct, then the lift, drag and weight of the duct create additional pitching moments as well. Then the total pitching moment about the tilting axis $(o z)$ in general form is:

$\Sigma M_{o}=M_{z}+(L \cos \alpha+D \sin \alpha) h_{a c}+W \bar{h}_{c g}=q_{\infty} S c C_{M}+(L \cos \alpha+D \sin \alpha) h_{a c}+W \bar{h}_{c g}$

Where, $h_{a c}$ is the distance from the tilting axis to the aerodynamic centre, $\bar{h}_{c g}$ is the horizontal distance from the tilting axis to the centre of gravity of the duct, and $W$ is the duct weight. 
2- When a fan is considered, the fan thrust force has components in both $x$ and $y$ directions. Also, due to the pressure difference between the two sides of the fan, the pressure distribution around the ducted fan is not the same as the pressure distribution around the duct without a fan. By tilting the ducted fan, the pressure distribution is changing around the duct and causes the changes in the aerodynamic forces and pitching moment as well.

Due to the presence of the rotor, a portion of the freestream flow has to change its direction to pass through the duct. Changes in the momentum of the freestream flow create ram drag. Based on the mass flow rate through the duct, freestream velocity and AoA, the ram drag can be expressed as [22]:

$D_{\text {ram }}=\dot{m} U \sin \alpha=\rho A V_{\text {ind }} U \sin \alpha$

This shows ram drag is zero if the ducted fan is parallel to the incoming flow (then $A o A=0$ ) or if the ducted fan is in hover with no crosswind (then $U=0$ ). Ram drag is perpendicular to the thrust line and normally generates a nose-up duct pitching moment based on the distance between the tilting axis of the duct and the centre of pressure of the shrouded rotor (which is about $0.75 \mathrm{R}$ further above the corresponding location for the open rotor [23]). This additional pitching moment can be expressed as:

$M_{\text {ram }}=D_{\text {ram }} \times h_{\text {ram }}$

Where, $h_{\text {ram }}$ is the distance from the tilting axis to the centre of pressure of the duct flow above the rotor plane. The resultant forces and moments about the tilting axis for any arbitrary AoA can now be expressed as [23]:

$\Sigma F_{x}=D_{\text {duct }}+D_{\text {frame }}+D_{\text {ram }} \sin \alpha-T \cos \alpha$

$\Sigma F_{y}=L_{\text {duct }}+D_{\text {ram }} \cos \alpha+T \sin \alpha-W$

$\Sigma M_{o}=M_{d u c t}+\left(L_{d u c t} \cos \alpha+D_{d u c t} \sin \alpha\right) h_{a c}+D_{\text {ram }} h_{r a m}+W \bar{h}_{c g}$ 
Due to the changes in AoA and flow separation around the duct lip in higher AoAs, the velocity of incoming flow to the duct intake is not constant and this causes the changes in the thrust force and pressure distribution around the duct. The change in the pressure distribution around the ducted fan is significant, and the calculated aerodynamic forces and pitching moment of the ducted fan are not comparable with those for the duct without a fan.

The ANSYS-Fluent software calculates the ducts' lift, drag and pitching moment and their coefficients by solving pressure distribution for any selected surface of the models. The tilting axis, which passes through the centre of the duct, is the reference axis for calculating all pitching moments. Thrust, ram drag and its related pitching moment are calculated using related equations and added to the resultant aerodynamic forces and pitching moment of the models.

\subsection{Turbulence modeling for ducted fans in crosswind}

Turbulence modeling is an important issue in CFD modeling of ducted fans in crosswind. The possible flow separation at the windward duct lip in high angled crosswind, combined with a wide range of tilting angle for ducted fans, provides non-uniform aerodynamic forces and pitching moment and a highly turbulent condition around the ducted fans $[8,10]$. The standard $k-\varepsilon$ turbulence modeling was used in this study for CFD simulations over ducts without a fan/rotor, and the RNG (Re-Normalisation Group) $k-\varepsilon$ turbulence modeling was used for simulations over ducted fans due to weak to moderate swirling flow (with Swirling No. $<0.5$ ).

The standard $k-\varepsilon$ turbulence modeling is a semi-empirical model [24]. Two model transport equations describe the turbulence kinetic energy $(k)$ and its dissipation rate $(\varepsilon)$ in this model. The most important assumptions for derivation of the standard $k-\varepsilon$ turbulence modeling is that the 
flow is fully turbulent, and the effects of molecular viscosity are negligible. Then, the standard $k-\varepsilon$ turbulence modeling is valid for fully turbulent flows.

The RNG $k-\varepsilon$ turbulence modeling has the similar form of the standard $k-\varepsilon$ turbulence modeling [25]. It has additional term in its dissipation rate $(\varepsilon)$ equation which improves the accuracy for rapidly strained flows. As the RNG theory provides an analytically derived differential formula for effective viscosity, then this model is suitable for low Reynolds number effects. Also the effect of swirl on turbulence is included in this model, which improves the accuracy for swirling flow as can be seen at the exit of ducted fans in this research. More details for selecting turbulence modeling are presented in the Appendix A.

The standard $k-\varepsilon$ turbulence modeling has been successfully used by other researchers such as Akturk et al. [11] to study a 5-inch ducted fan for vertical or short take-off and landing UAV applications and also by Camci and Akturk [12] to study a double ducted fan.

\subsection{Mesh selection for CFD modeling}

To find the best number of cells, three different mesh sizes were considered for symmetrical duct without fan as described in Table 3.1. The ANSYS-Fluent calculated lift, drag and pitching moment coefficients for these three mesh sizes in static conditions at different AoAs. The CPU time was measured for computing aerodynamic coefficients of each mesh type.

Table 3.1: The specifications of different meshes of symmetrical duct without fan including No. of volumetric cells and CPU time per step for computing aerodynamic coefficient

\begin{tabular}{|l|c|c|c|}
\hline \multicolumn{1}{|c|}{ No. of intervals for each edge location } & Coarse Mesh & Moderate Mesh & Fine Mesh \\
\hline Outer edge for external body of duct (chord) & 50 & 100 & 200 \\
\hline Inner edge for internal body of duct (chord) & 50 & 100 & 200 \\
\hline Duct's inlet circular edge (at leading edge) & 150 & 300 & 600 \\
\hline Duct's outlet circular edge (at trailing edge) & 150 & 300 & 600 \\
\hline Half-circle edge of spherical boundary & 25 & 50 & 100 \\
\hline Total No. of volumetric cells & 204,326 & 849,339 & $3,867,760$ \\
\hline CPU time /step (sec) & 1.03 & 5.14 & 25.83 \\
\hline
\end{tabular}


The mean diameter at the inlet and outlet of the ducts are equal to the duct's chord length set to $22 \mathrm{~cm}$, which is the size of the actual models in this research. The free stream velocity is set to $\mathrm{U}=15 \mathrm{~m} / \mathrm{s}$ at the inlet boundary of domain. As expected, the process time for mesh with higher number of cells was too long. The mesh with 849,339 cells is in moderate calculation time and its results are reasonably close to the finer mesh, as shown in Figure 3-8.

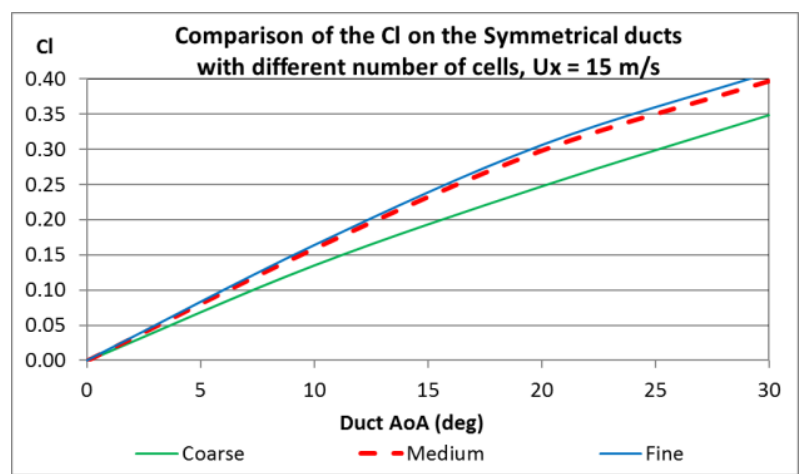

(a)

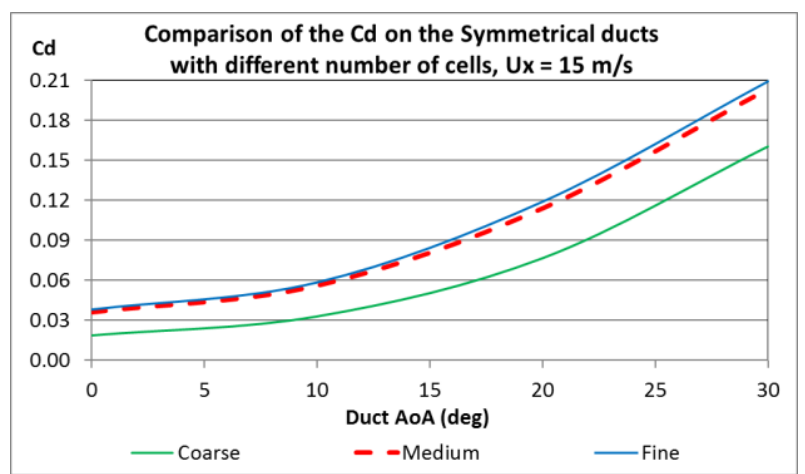

(b)

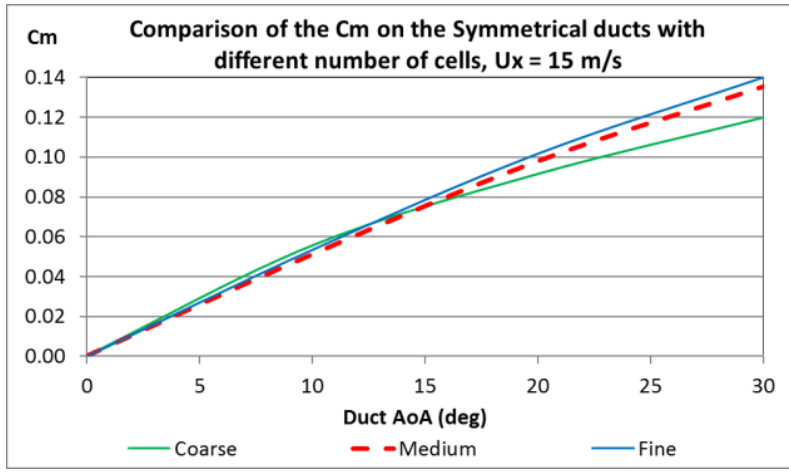

(c)

Figure 3-8: Comparison of aerodynamic coefficients for different mesh sizes for symmetrical duct in the range of $0^{\circ} \leq A o A \leq 30^{\circ}$ : (a) $C_{l}$, (b) $C_{d}$, and (c) $C_{m}$

The grid independence also examined by calculating lift coefficient for the meshes were introduced in the Table 3.1 among four more meshes (with cell numbers close to the moderate mesh) in the steady state condition with $A o A=30^{\circ}$ and $\mathrm{U}=15 \mathrm{~m} / \mathrm{sec}$ (see Figure 3-9). Based on the required precision level and computation time limitations for different mesh sizes, the moderate mesh is selected for CFD simulations of the symmetrical duct. In addition, the specification of moderate mesh is used to create other models for this study (asymmetrical duct without fan/rotor, 
and symmetrical and asymmetrical ducted fans), which are listed in Table 3.2. The details of geometry, number of spatial and interval sizes for different edges of the meshes for both ducts with/without fans are presented in Appendix B.

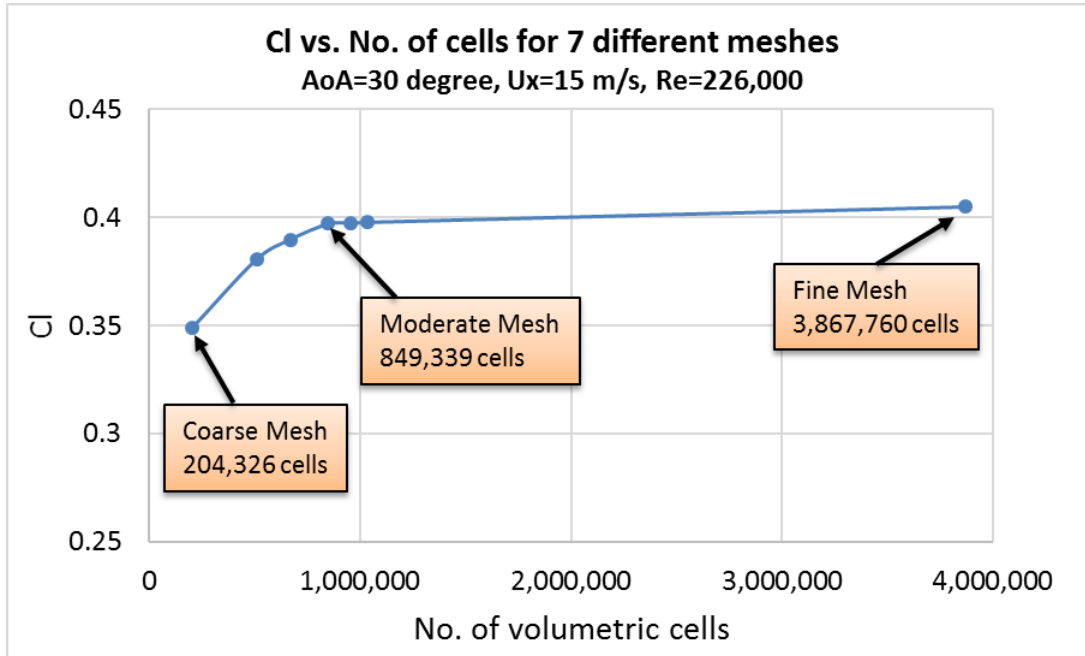

Figure 3-9: Comparing $C_{l}$ for seven meshes with different No. of cells in stationary state $(A o A=$ $\left.30^{\circ}, U_{x}=15 \mathrm{~m} / \mathrm{sec}, \operatorname{Re}=226,000\right)$

Table 3.2: Number of volumetric cells for different mesh sizes in this research

\begin{tabular}{|l|c|}
\hline \multicolumn{1}{|c|}{ Model } & No. of volumetric cells \\
\hline Symmetrical duct without fan/rotor & 849,339 \\
\hline Asymmetrical duct without fan/rotor & 830,982 \\
\hline Symmetrical ducted fan & $1,068,942$ \\
\hline Asymmetrical ducted fan & $1,142,536$ \\
\hline
\end{tabular}

\subsection{Proposed method to calculate the inlet flow velocity to the ducted fans}

The proposed method for calculating the inlet flow velocity to the ducted fans is based on the actuator disk model [18] and the assumption of constant power delivered to the propeller to change the linear momentum of the inlet flow to the rotor plane in each case. First of all, this method provides a trial and error procedure to calculate the exact value for induced velocity $\left(V_{\text {ind }}\right)$ in cruise condition by using ANSYS-Fluent software. Then, the assumption of constant induced power delivered to the propeller in all AoAs provides a cubic polynomial equation of $V_{\text {ind }}$. Therefore, as the induced velocity $\left(V_{\text {ind }}\right)$ is a function of AoA, so are ducted fan's thrust and ram drag. 


\section{Step 1: Finding $V_{\text {ind }}$ in the cruise condition}

The balance of forces in a steady level flight for proposed VTOL UAV in the $x$-direction can be described by the following equation:

$$
\Sigma F_{x}=D_{\text {total }}-T=0 \quad \text { (in steady level flight condition) }
$$

As the total drag for proposed VTOL UAV is the combination of body drag (drag from fuselage, wing and tail) and two ducted fans drag, then:

$$
\Sigma F_{x}=D_{b o d y}+D_{d u c t}-T=0 \quad \text { (in steady level flight condition) }
$$

Similarity between steady level flight condition and wind tunnel tests in this research, $U=$ $15 \mathrm{~m} / \mathrm{s}$ and $A o A=0^{\circ}$, results in replacing $D_{b o d y}$ (for the proposed VTOL UAV) with $D_{\text {frame }}$ (for the tested models), as explained in the Appendix E. Then, equation (3.30) can be modified for only one ducted fan as:

$$
\Sigma F_{x}=D_{\text {frame }}+D_{\text {duct }}-T=0 \quad \text { (at zero AoA) }
$$

Using thrust calculation for ducted fans in cruise mode [18] can be employed to find the induced velocity $\left(V_{\text {ind }}\right)$ at zero AoA as follows:

$T=\rho A V_{\text {ind }}(w-U)=\rho A V_{\text {ind }}\left(V_{\text {ind }} / a_{w}-U\right)$

where, $w$ is downwash velocity at the duct exit and can be written as $w=V_{\text {ind }} / a_{w}$. The wake contraction parameter, $a_{w}$, was introduced in sec. 3.1 and can be calculated for both ducts as:

$a_{w}=\frac{V_{\text {ind }}}{w}=\frac{V_{\text {ind }}}{w} \times \frac{\rho A_{\text {inlet }}}{\rho A_{\text {wake }}} \times \frac{A_{\text {wake }}}{A_{\text {inlet }}}=\frac{\dot{m}}{\dot{m}} \times \frac{A_{\text {wake }}}{A_{\text {inlet }}}=\frac{A_{\text {wake }}}{A_{\text {inlet }}}$

Using duct specifications (see Table 3.3):

$a_{w}=\frac{A_{\text {wake }}}{A_{\text {inlet }}}=\left(\frac{R_{\text {out }}}{R_{\text {in }}}\right)^{2}=\left(\frac{11 \mathrm{~cm}}{10.25 \mathrm{~cm}}\right)^{2}=1.1517$

Equation (3.31) can be satisfied only by knowing the exact value of the induced velocity $\left(V_{\text {ind }}\right)$. Also it is necessary to find $D_{\text {frame }}, D_{d u c t}$, and thrust of the ducted fan for both symmetrical and 
asymmetrical models. The $D_{\text {frame }}$ can be measured experimentally in the wind tunnel but thrust and $D_{\text {duct }}$ should be computed by knowing the exact value of $V_{\text {ind }}$. The exact value of $V_{\text {ind }}$ can be found by trial and error method, guessing an initial value for $V_{\text {ind }}$, and using ANSYS-Fluent software to compute $D_{d u c t}$. Then, thrust can be calculated by knowing: $T=D_{\text {frame }}+D_{\text {duct }}$ and solving equation (3.32) to find new $V_{\text {ind }}$. If the difference between initial guess and new calculated $V_{\text {ind }}$ is more than a specific error, then this procedure should be repeated by entering the new calculated $V_{\text {ind }}$ as initial guess to the ANSYS-Fluent software. The ANSYS-Fluent software initial setup for each ducted fan is listed in Table 3.4. After several runs of the ANSYS-Fluent, the convergence was observed for $V_{\text {ind }}$ and there was no difference between initial guess and final calculated $V_{\text {ind }}$. It was also observed that maximum number of required runs of the ANSYS-Fluent software for this convergence were five runs for each ducted fan. The final induced velocities at zero AoA were found and listed in Table 3.5.

Table 3.3: Specifications of symmetrical and asymmetrical ducted fans

\begin{tabular}{|l|c|}
\hline \multicolumn{1}{|c|}{ Specifications } & Both symmetrical and asymmetrical \\
\hline Inlet mean diameter & $0.220 \mathrm{~m}$ \\
\hline Outlet mean diameter & $0.220 \mathrm{~m}$ \\
\hline Duct length (chord) & $0.220 \mathrm{~m}$ \\
\hline Average surface area of duct & $0.152053084 \mathrm{~m}^{2}$ \\
\hline Inner diameter of duct & $0.205 \mathrm{~m}$ \\
\hline Propeller diameter & $0.2032 \mathrm{~m}$ \\
\hline Propeller tip clearance & Close to $1 \mathrm{~mm}$ \\
\hline Rotor disk area & $0.03243 \mathrm{~m}^{2}$ \\
\hline
\end{tabular}

Table 3.4: Initial setup for the ANSYS-Fluent software with models in zero AoA

\begin{tabular}{|l|l|}
\hline \multicolumn{1}{|c|}{ Items } & \multicolumn{1}{c|}{ Entered values } \\
\hline Freestream velocity and duct's AoA & $U=15 \mathrm{~m} / \mathrm{s}, A o A=0^{\circ}$ \\
\hline Initial value for inlet flow velocity & $V_{\text {ind }}=20 \mathrm{~m} / \mathrm{s}$ \\
\hline Turbulence modeling & $\mathrm{k}-\varepsilon$ Re-Normalisation Group \\
\hline Max. No. of iterations for running software in each case & 800 \\
\hline
\end{tabular}


Table 3.5: Final induced velocities, thrust and induced power for each duct at zero AoA

\begin{tabular}{|l|c|c|}
\hline \multicolumn{1}{|c|}{ Parameter } & Symmetrical ducted fan & Asymmetrical ducted \\
\hline Induced velocity, $V_{\text {ind }}$ & $21.25 \mathrm{~m} / \mathrm{s}$ & $21.4 \mathrm{~m} / \mathrm{s}$ \\
\hline Thrust, $T$ & $2.9133 \mathrm{~N}$ & $3.0446 \mathrm{~N}$ \\
\hline Induced power, $P$ & 61.908 watts & $65.154 \mathrm{watts}$ \\
\hline
\end{tabular}

\section{Step 2: Finding $V_{\text {ind }}$ for the other AoAs}

The main flow velocity can be divided into two components relative to the rotational coordinates attached to the ducted fans. The normal component toward rotor plane is $U \cos \alpha$, which can affect the ducted fan's thrust. Therefore, it is necessary to replace $U$ with $U \cos \alpha$ in equation (3.32) to be valid for all AoAs. To calculate $V_{\text {ind }}$ for all other AoAs, the assumption of $P=T . V_{\text {ind }}=$ const. can be used with modification of equation (3.32) as follows:

$T=\rho A V_{\text {ind }}\left(V_{\text {ind }} / a_{w}-U \cos \alpha\right)$

$P=T . V_{\text {ind }}=\rho A V_{\text {ind }}^{2}\left(V_{\text {ind }} / a_{w}-U \cos \alpha\right)$

which results in:

$\frac{\rho A}{a_{w}} V_{i n d}^{3}-\rho A U \cos \alpha V_{i n d}^{2}-P=0$

or: $V_{\text {ind }}^{3}-a_{w} U \cos \alpha V_{\text {ind }}^{2}-\frac{P a_{w}}{\rho A}=0$

All parameters in equation (3.37) are known and the only unknown is $V_{\text {ind }}$. Equation (3.37) is a cubic polynomials and can be solved using ordinary methods. After finding $V_{\text {ind }}$ for each AoA, thrust and ram drag can be calculated using equations (3.34) and (3.25).

The flow chart in Figure 3-10 shows the summary of the procedure to find the power in the cruise mode and also $V_{\text {ind }}$ in different AoAs for each ducted fan.

The calculated induced velocities for each ducted fan are shown in Table 3.6 and Figure 3-11. These induced velocities were used for running CFD simulations and the results showed satisfactory agreement between experimental and CFD modeling. 


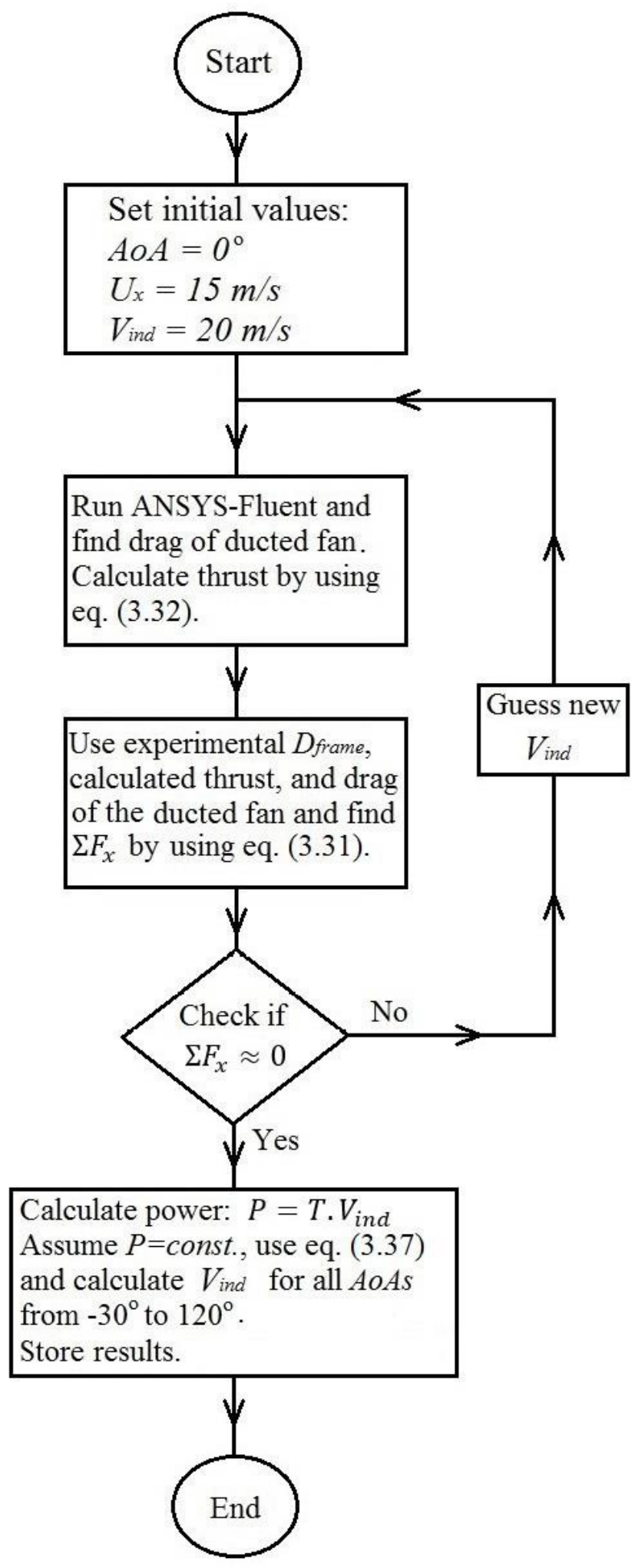

Figure 3-10: The flowchart for finding power in cruise mode and also $V_{\text {ind }}$ in all different AoAs for each ducted fan 
Table 3.6: Calculated $V_{\text {ind }}$ and Thrust for symmetrical and asymmetrical ducted fans

\begin{tabular}{|c|c|c|c|c|c|c|}
\hline \multirow{2}{*}{$\begin{array}{c}\text { Alpha } \\
(\text { deg })\end{array}$} & \multicolumn{2}{|c|}{ Symmetrical ducted fan } & \multicolumn{3}{|c|}{ Asymmetrical ducted fan } \\
\cline { 2 - 7 } & $\begin{array}{c}\text { Power } \\
(\text { watts })\end{array}$ & $\begin{array}{c}V_{\text {ind }} \\
(\mathrm{m} / \mathrm{s})\end{array}$ & $\begin{array}{c}\text { Thrust } \\
(\mathrm{N})\end{array}$ & $\begin{array}{c}\text { Power } \\
(\text { watts })\end{array}$ & $\begin{array}{c}V_{\text {ind }} \\
(\mathrm{m} / \mathrm{s})\end{array}$ & $\begin{array}{c}\text { Thrust } \\
(\mathrm{N})\end{array}$ \\
\hline-30 & 61.908 & 19.622 & 3.155 & 65.154 & 19.786 & 3.293 \\
\hline-20 & 61.908 & 20.503 & 3.019 & 65.154 & 20.659 & 3.154 \\
\hline-10 & 61.908 & 21.060 & 2.940 & 65.154 & 21.211 & 3.072 \\
\hline 0 & 61.908 & 21.250 & 2.913 & 65.154 & 21.400 & 3.045 \\
\hline 10 & 61.908 & 21.060 & 2.940 & 65.154 & 21.211 & 3.072 \\
\hline 20 & 61.908 & 20.503 & 3.019 & 65.154 & 20.659 & 3.154 \\
\hline 30 & 61.908 & 19.622 & 3.155 & 65.154 & 19.786 & 3.293 \\
\hline 40 & 61.908 & 18.486 & 3.349 & 65.154 & 18.659 & 3.492 \\
\hline 50 & 61.908 & 17.183 & 3.603 & 65.154 & 17.367 & 3.752 \\
\hline 60 & 61.908 & 15.814 & 3.915 & 65.154 & 16.008 & 4.070 \\
\hline 70 & 61.908 & 14.475 & 4.277 & 65.154 & 14.677 & 4.439 \\
\hline 80 & 61.908 & 13.239 & 4.676 & 65.154 & 13.447 & 4.845 \\
\hline 90 & 61.908 & 12.153 & 5.094 & 65.154 & 12.361 & 5.271 \\
\hline 100 & 61.908 & 11.230 & 5.513 & 65.154 & 11.438 & 5.696 \\
\hline 110 & 61.908 & 10.469 & 5.913 & 65.154 & 10.673 & 6.105 \\
\hline 120 & 61.908 & 9.852 & 6.284 & 65.154 & 10.053 & 6.481 \\
\hline
\end{tabular}

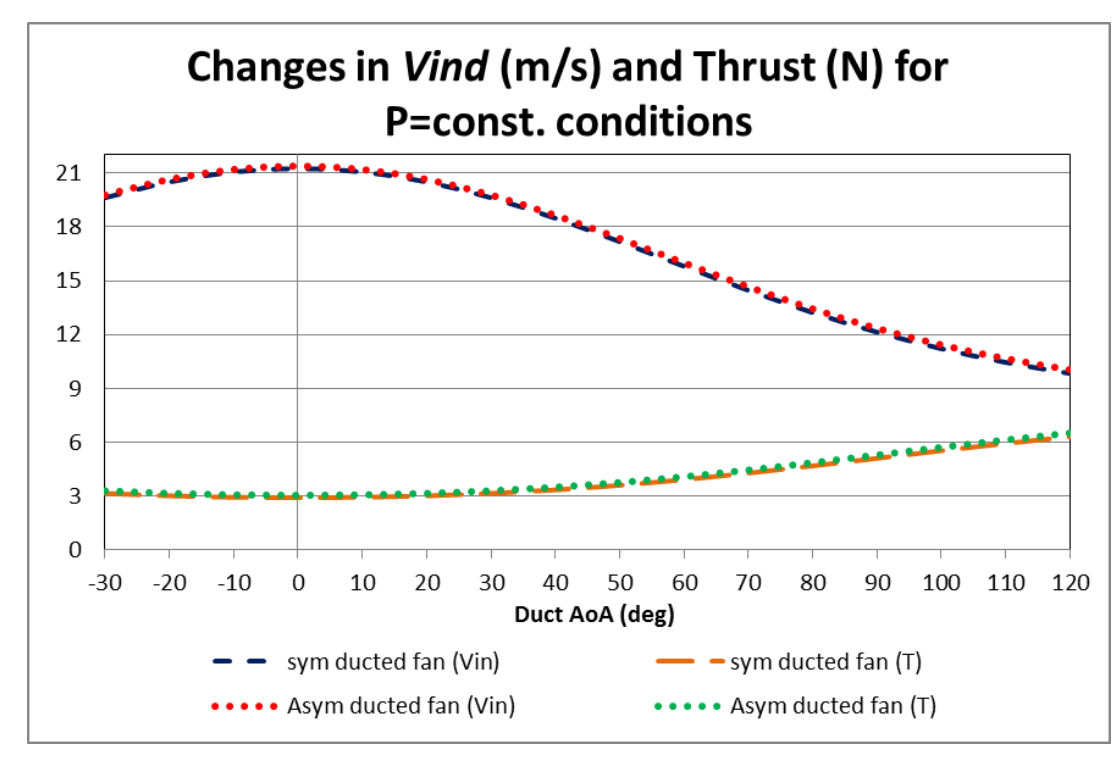

Figure 3-11: Variations of induced velocity $\left(V_{\text {ind }}\right)$ and thrust $(T)$ in the range of $-30^{\circ} \leq \alpha \leq$ $120^{\circ}$ for symmetrical and asymmetrical ducted fans with assumption of $P=$ const.

To find the $V_{\text {ind }}$ for an actual model of VTOL UAV, it is necessary to estimate the total drag of the VTOL UAV (summation of the induced drag and the profile drag for fuselage, wing, and tail) 
in the steady level flight condition (cruise mode). Then, equation (3.31) can be used by replacing $D_{\text {frame }}$ with the estimated $D_{\text {total }}$ for the actual model of VTOL UAV. Next, by following the procedure in Figure 3-10, it helps to find the power and also the $V_{\text {ind }}$ for all AoAs.

\subsection{Verification of the CFD method}

The verification of the CFD modeling are presented in the Chapter 4 (Sections 4.2 and 4.6) and as a preapproval of the method in this research, the velocity distribution of the flow around the symmetrical ducted fan in crosswind was simulated in this section. The selected parameters for simulation were based on the CFD simulations by Fleming et al. [9] and their results are illustrated in Figure 3-12. The results from this research are shown in Figure 3-13.
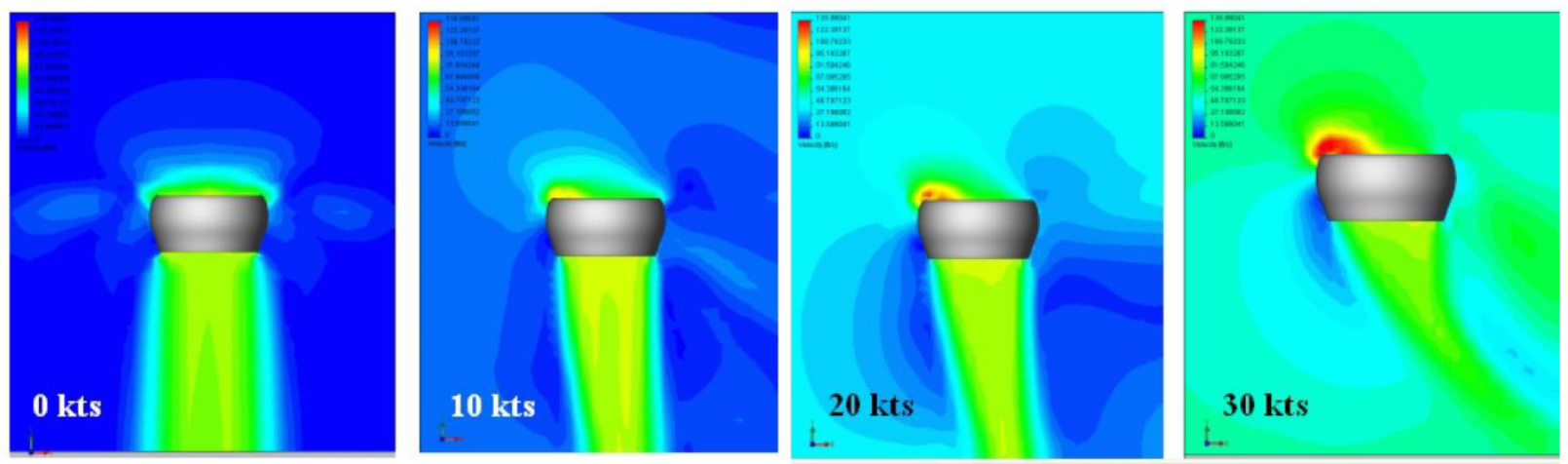

Figure 3-12: Duct wake bending with increasing crossflow. Progression is from 0 to 30 knots (equal to 0 to $15.43 \mathrm{~m} / \mathrm{s}$ ), 12 psf disks loading modeled (equal to $574.6 \mathrm{~N} / \mathrm{m}^{2}$ or $15.314 \mathrm{~m} / \mathrm{s}$ of inlet flow velocity to the duct). Range of color contours is 0 to $136 \mathrm{ft} / \mathrm{sec}$. [9]

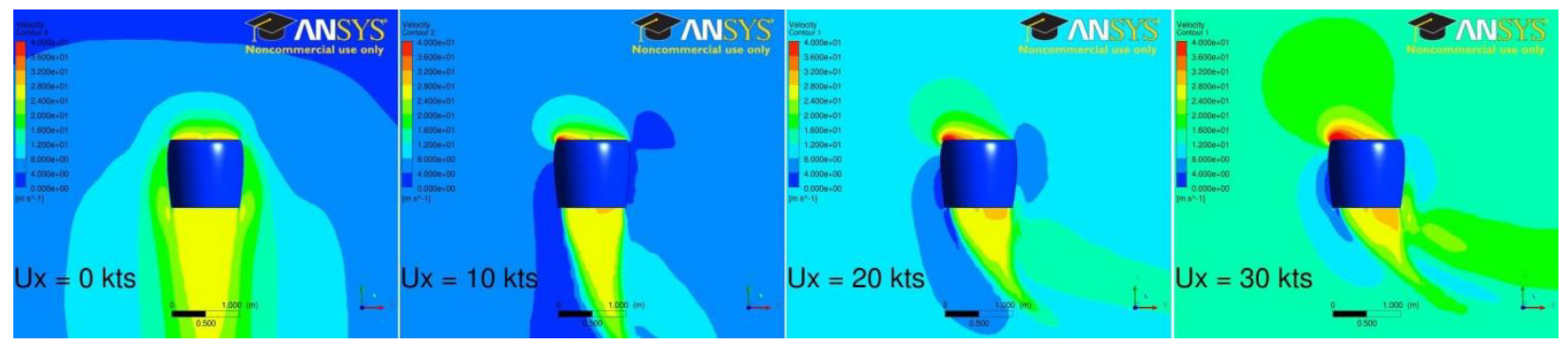

Figure 3-13: Duct wake bending with increasing crossflow in this research. Progression is from 0 to 30 knots (equal to 0 to $15.43 \mathrm{~m} / \mathrm{s}$ ), 12 psf disk loading modeled (equal to $574.6 \mathrm{~N} / \mathrm{m}^{2}$ or $15.314 \mathrm{~m} / \mathrm{s}$ of inlet flow velocity to the duct). Range of color contours is 0 to $40 \mathrm{~m} / \mathrm{sec}$. 
Both simulations predicted an unusual increase in the velocity over the windward duct lip, which creates an unbalanced lift force at the inlet of the duct and generates pitching moment. The similarity of the results between the two simulations is the first verification for the CFD method of this research.

\subsection{Aerodynamics of tilting ducted fans}

The large increment in the AoA has extensive effects on the flow behaviour around a ducted fan. Assume a ducted fan with high AoA in the freestream with a velocity of $U_{x}$ as illustrated in Figure 3-14. One part of oncoming flow has to enter the ducted fan normal to the rotor plane. The change in the direction of the oncoming flow is equal to the ducted fan's AoA. At the exit of the ducted fan, the outgoing jet impacts the mainstream flow with the angle equal to the ducted fan's AoA. Sharp turns of the flow into the rotor plane and at the exit of the duct in higher AoAs result in the unsteady flow separation around duct lip and strong swirling vorticities at the duct exit [26].

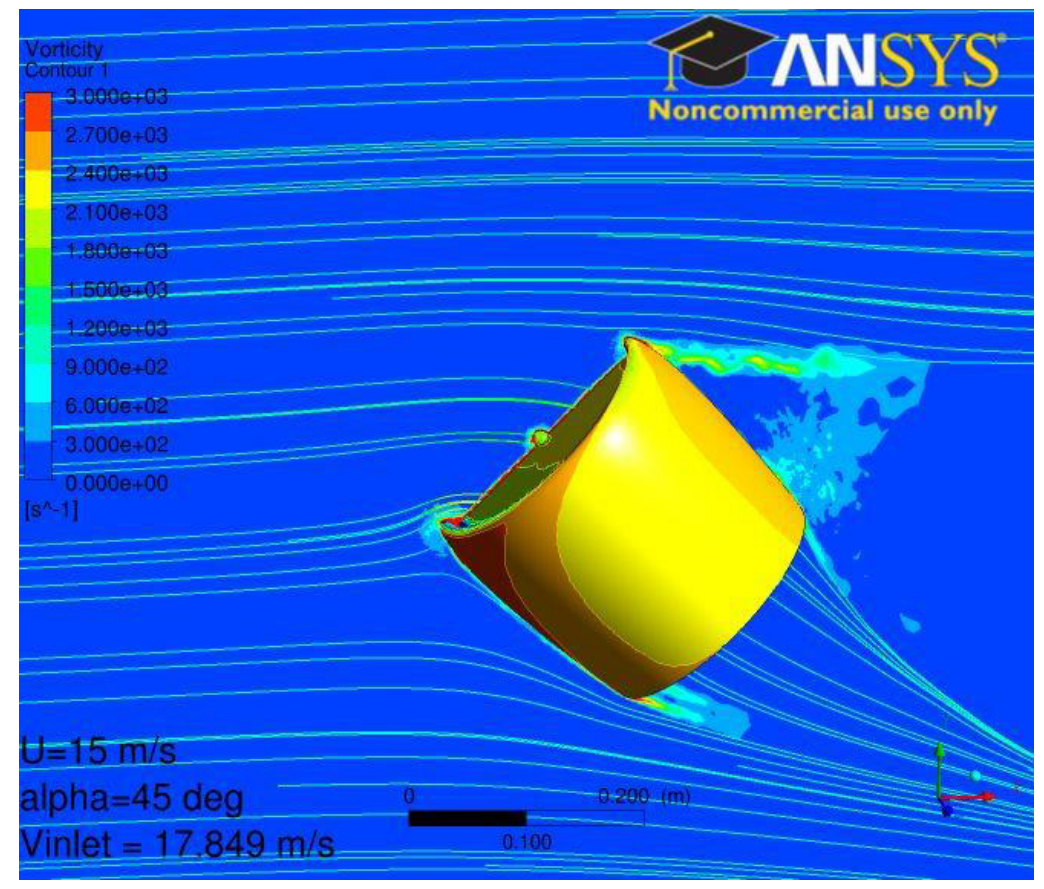

Figure 3-14: Flow streamlines combined with contour of vorticity on the xy plane around the symmetrical ducted fan 
More than that, in higher AoAs, the duct body acts the same as a cylinder and flow separation occurs behind the duct [27]. All these are reasons for vortex shedding and large disturbances in the pressure distribution around the duct in higher AoAs. These large disturbances cause cyclic changes in the pressure forces around the duct in higher AoAs, especially close to the duct lips and rotor plane.

The rate of change in the $\operatorname{AoA}(\omega=\dot{\alpha}=d \alpha / d t)$ is another important parameter affecting the occurrence of the disturbances in the pressure distribution around a tilting ducted fan. Although the disturbance in the pressure distribution in the stationary condition is a function of AoA, in the transition condition it will be affected by the rate of change in the AoA too. The larger rate of changes in the $\operatorname{AoA}(\omega)$ will cause more unexpected fluctuations in the pressure distribution during transition periods. The fluctuations in the pressure distribution causes sudden changes in the aerodynamic forces, and this will be shown in the next chapter by using CFD simulations.

\subsection{Tilting ducted fan VTOL UAV in transition condition}

An important part of this research is the study of transition between cruise mode and hover of the proposed VTOL UAV. Maintaining in a level flight during transition is a necessity, but it can be difficult due to rapid changes in the VTOL UAV's velocity and the aerodynamic coefficients of the tilting ducted fans. These rapid changes generate transitional pitching moment and affect the AoA of the VTOL UAV. The final result could be a rapid ascending combined with stall condition or a rapid descending.

The focus of this section is on the stability of the proposed VTOL UAV during transition from cruise mode to hover by using two different types of tilting ducted fans (one symmetrical and one asymmetrical). 
The resultant aerodynamic forces and pitching moment acting on the proposed VTOL UAV in transition can be found by knowing the speed of VTOL UAV and the characteristics of each component such as wing, horizontal and vertical stabilizers, fuselage and tilting ducted fans. In this research, the aerodynamic coefficients of fixed components were assumed as constant parameters. Figure 3-15 shows the selected coordinate system and aerodynamic forces acting on the proposed VTOL UAV in transition between cruise condition and hover.

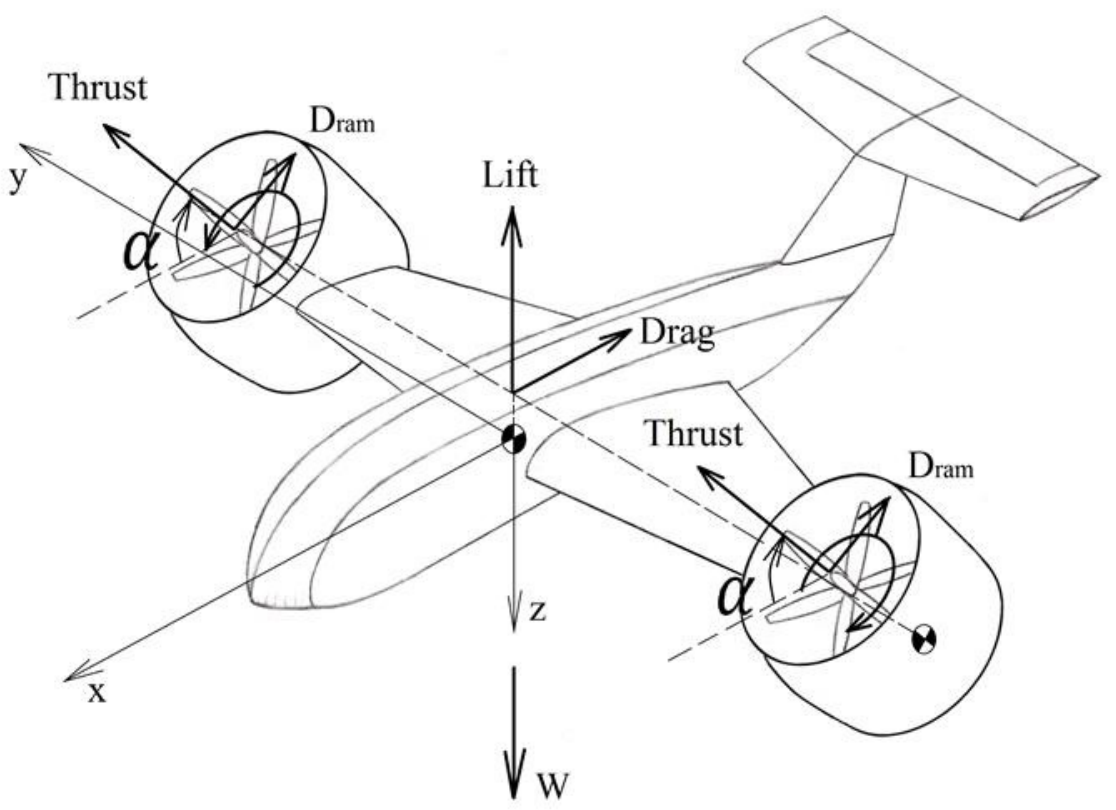

Figure 3-15: Coordinate system and aerodynamic forces acting on the proposed typical VTOL UAV

Assuming that the aerodynamic centre of the wing is exactly located above the centre of the gravity of the proposed VTOL UAV with the vertical distance of $h_{a c}$, and assuming zero lift and pitching moment coefficients for the fuselage, then, the general form of the resultant aerodynamic forces and pitching moments in a level flight during transition can be expressed as follows:

$$
\begin{aligned}
& \Sigma F_{x}=2\left(T \cos \alpha-D_{\text {ram }} \sin \alpha-D_{\text {duct }}\right)-D_{\text {wing }}-D_{\text {fuselage }}-D_{v s}-D_{h s} \\
& \Sigma F_{z}=W-L_{\text {wing }}-2\left(T \sin \alpha+D_{\text {ram }} \cos \alpha+L_{\text {duct }}\right)
\end{aligned}
$$




$$
\Sigma M_{y}=2 M_{d u c t}+M_{w i n g}+D_{w i n g} h_{a c}+D_{v s} h_{v s}+D_{h s} h_{h s}+L_{h s} l_{h s}
$$

In equation (3.40), $M_{\text {duct }}$ is the summation of several pitching moments from different forces as follows:

$M_{d u c t}=D_{d u c t}\left(h_{a c}+l_{d u c t} \sin \alpha\right)+L_{d u c t} l_{d u c t} \cos \alpha+\left(D_{\text {ram }} \sin \alpha-T \cos \alpha\right) h_{a c}+$

$D_{\text {ram }} h_{\text {ram }}+\frac{1}{2} \rho S_{\text {duct }} c_{\text {duct }} U^{2} C_{m_{\text {duct }}}$

Lift, drag, and pitching moments of each component can be calculated by well-known equations as following:

$$
\begin{aligned}
& L=\frac{1}{2} \rho S U^{2} C_{L} \\
& D=\frac{1}{2} \rho S U^{2} C_{D} \\
& M=\frac{1}{2} \rho S c U^{2} C_{M}
\end{aligned}
$$

Thrust and momentum drag of the ducted fans are functions of free stream velocity and local angle of attack, and were described before in the equations (3.34) and (3.25). Substitution equations (3.34) and (3.25) into equation (3.40) results in:

$$
\Sigma F_{z}=W-L_{w i n g}-2\left(\rho A V_{i n d}^{2} \sin \alpha / a_{w}+L_{d u c t}\right)
$$

The necessity of level flight for proposed VTOL UAV during transition defines a new constraint of $\Sigma F_{z}=0$, which means there should not be any acceleration in z-direction during transition. This assumption helps us to set up a trial and error method for calculating the proposed VTOL UAV velocity $(\mathrm{U})$ and induced velocity to the rotor disk $\left(\mathrm{V}_{\text {ind }}\right)$ based on the tilting rate $(\omega)$ and time during transition process.

The following assumptions have been made to have a level flight during transition from cruise mode to hover:

1- The transition starts by reduction in the thrust of two ducted fans. 
2- At the same time, two ducted fans start tilting about their tilting axis with constant tilting rate $(\omega)$.

3- The thrust of the rotors and the angle of tail's elevator were selected as the only controllable parameters during transition.

Reduction in the thrust of ducted fans is necessary, as the summation of the wing's lift and vertical component of the thrust $(T \sin \alpha)$ causes a sudden ascending of the VTOL UAV at the starting of the transition. Tilting rate for ducted fans should be selected at the first step (e.g. $\omega=$ $10 \mathrm{deg} / \mathrm{sec}$ ). As the ducted fans start tilting and by reduction in their thrust, the reduction in the VTOL UAV's velocity affects lift, drag and pitching moments of different components of the VTOL UAV. Necessity of remaining in the level flight means any additional generated pitching moments by other components should be canceled by using tail's elevator, and this will guaranty no changes in the AoA for VTOL UAV. The achievement of the hover condition needs tilting angle of the ducted fans to be increased to higher angles than $90^{\circ}$ to produce essential braking force for shorter travelled time and distance during transition. In this case, when two ducted fans have tilting angle higher than $90^{\circ}$ and just before reaching to $U=0 \mathrm{~m} / \mathrm{s}$, two ducted fans must tilt backward to $\alpha=90^{\circ}$ with a negative tilting rate (e.g. $\omega^{\prime}=-10 \mathrm{deg} / \mathrm{sec}$ ) to get into stable hover condition. 


\section{RESULTS AND DISCUSSION}

\subsection{CFD simulations for symmetrical and asymmetrical ducts without fans}

To have a better comparison between symmetrical and asymmetrical ducts, simulations are first performed for ducts without fans. A symmetrical and an asymmetrical ducts are modeled as shown in Figure 4-1 and simulated by using standard $k-\varepsilon$ turbulence modeling in steady flow with $\mathrm{U}$ $=15 \mathrm{~m} / \mathrm{s}$ and tilting angles of $-30^{\circ} \leq A o A \leq 120^{\circ}$ (with $10^{\circ}$ increments in the AoA).

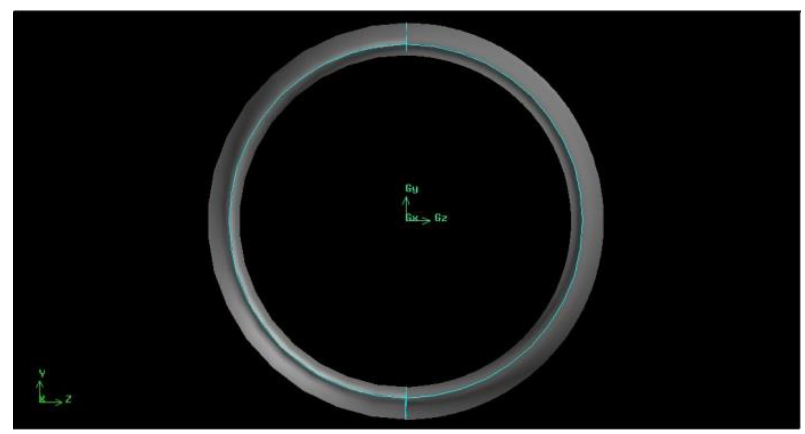

(a)

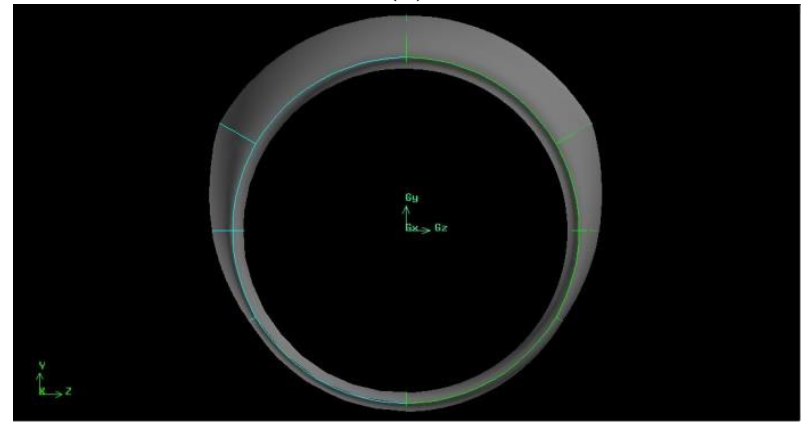

(c)

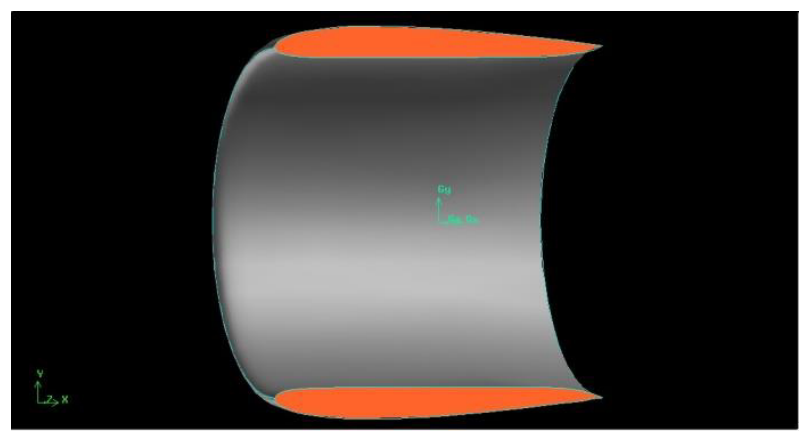

(b)

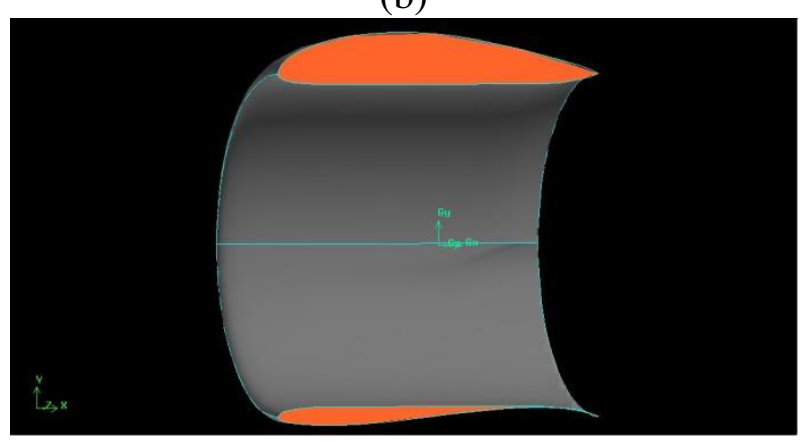

(d)

Figure 4-1: Two ducts without fans; symmetrical duct: (a) front view, (b) cross section in the xy plane and asymmetrical duct: (c) front view, (d) cross section in the xy plane

The aerodynamic coefficients of forces and pitching moments acting on two ducts without fans in stationary conditions are shown and compared in Figure 4-2. It can be seen that in the absence of the fans both ducts have the same trends for all aerodynamic coefficients. Also $C_{d}$ and $C_{m}$ of the symmetrical duct are very close to those for the asymmetrical duct. The most important result is obtained from the lift diagram shown in Figure 4-2a, which shows the asymmetrical duct has 
higher generated lift than the symmetrical duct. In these simulations, the range of lift coefficients for symmetrical and asymmetrical ducts are $-0.398 \leq C_{l_{\text {sym duct }}} \leq 0.398$ and $-0.332 \leq$ $C_{l_{\text {Asym duct }}} \leq 0.453$, respectively. The difference between two lift coefficients is noticeable and the asymmetrical duct has generated more lift (around 20\%) than the symmetrical duct for a wide range of tilting angle $\left(-30^{\circ} \leq A o A \leq 40^{\circ}\right)$. Generating lift by the external body of the asymmetrical duct can help to design and use smaller wing for a VTOL UAV, which can reduce overall drag and save more fuel (or electrical energy) to increase the range in the cruise mode.

As shown in Figure 4-2b, the range of drag coefficient by symmetrical and asymmetrical ducts are $0.0036 \leq C_{d_{\text {sym duct }}} \leq 0.4228$ and $0.0045 \leq C_{d_{\text {Asym duct }}} \leq 0.4325$, respectively. The minimum drag coefficient of the asymmetrical duct is only 0.0009 higher than that of the symmetrical duct in cruise mode $\left(A o A=0^{\circ}\right)$. Thus, using the asymmetrical duct has a very small increment in the profile drag due to the shape of external body.

As illustrated in Figure 4-2c, the asymmetrical duct has a small negative pitching moment coefficient in cruise mode $\left(A o A=0^{\circ}\right)$. The $C_{m_{\alpha}}$ is positive in the range of $-30^{\circ} \leq \alpha \leq 30^{\circ}$ as the tilting axis passes through the centre of the ducts' bodies, which is behind the aerodynamic centre of the ducts. Due to the stability and control of the proposed VTOL UAV, any generated pitching moments from tilting ducted fans should be cancelled by using the tail elevator during the transition between cruise and hover modes.

The contours of pressure around the ducts are shown in Figure 4-3. As seen, the lower surface of the asymmetrical duct has a locally higher pressure region relative to the symmetrical duct. Also, the upper surface of the asymmetrical duct has a locally lower pressure region relative to the symmetrical duct. 


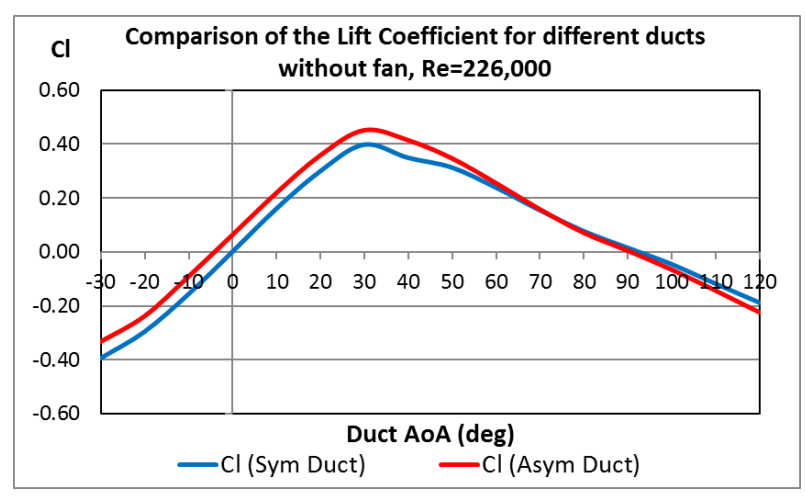

(a)

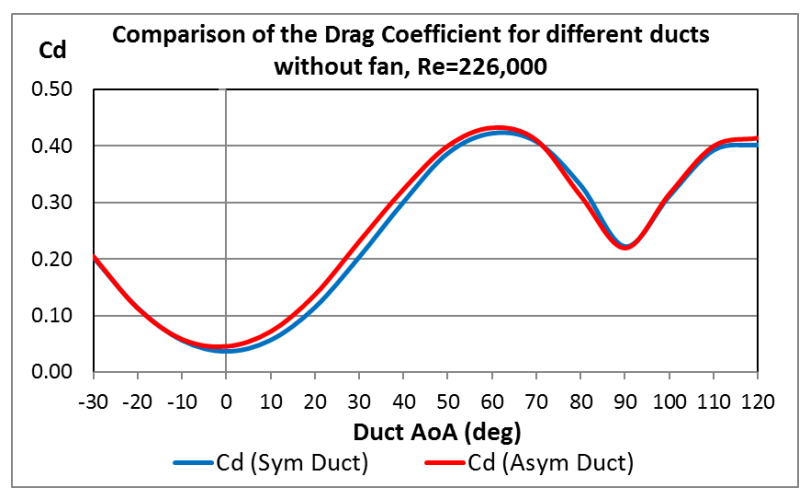

(b)

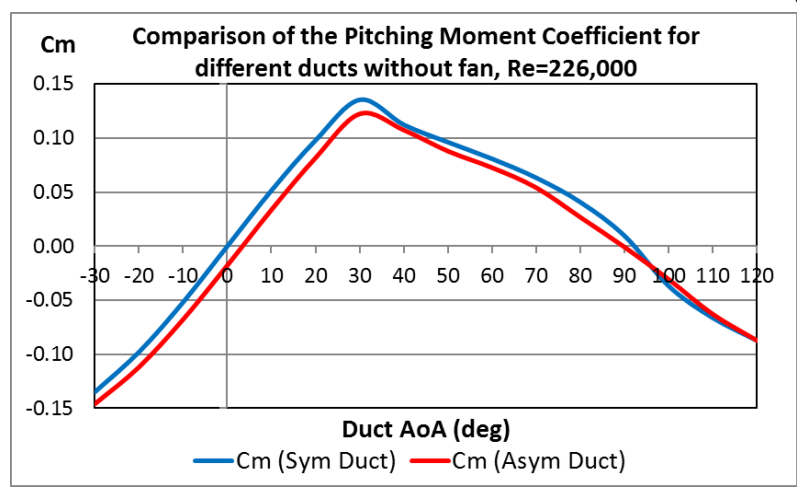

(c)

Figure 4-2: The variation of aerodynamic coefficients for symmetrical and asymmetrical ducts without fans (a) $C_{l}$, (b) $C_{d}$, and (c) $C_{m}$

The larger difference between the pressures on the lower and upper sides of the asymmetrical duct has the main role of generating additional lift in the cruise mode and higher AoAs. As expected, this mechanism can generate extra lift for a limited tilting motion of the asymmetrical duct relative to the symmetrical duct, in the range of $-30^{\circ} \leq A o A \leq 70^{\circ}$, as shown in Figure 4-2a.

The contours of pressure on the plane of symmetry around two ducts when $A o A=45^{\circ}$ are presented in Figure 4-4. The asymmetrical duct has a locally higher pressure region close to its lower surface due to its specific shape. 


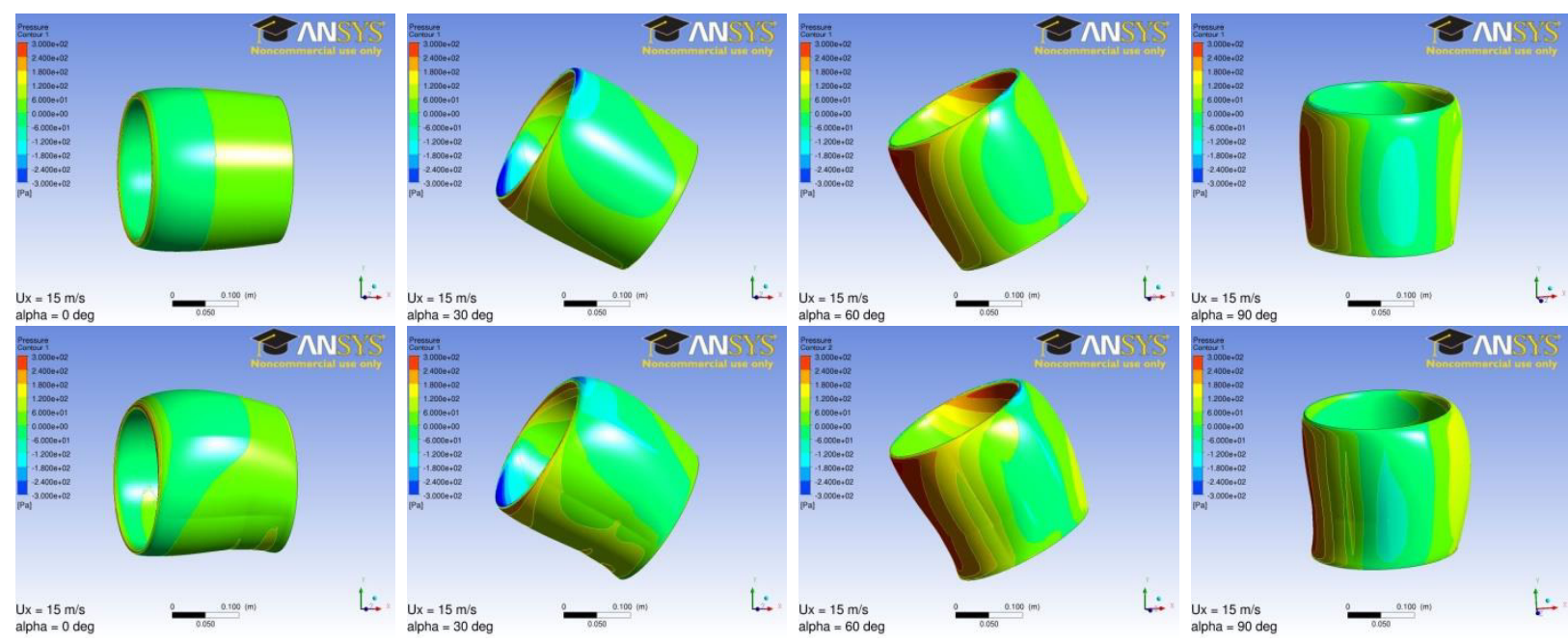

Figure 4-3: The contour of pressure around the symmetrical duct (top) and asymmetrical duct (bottom) without fans in steady flow $\left(\operatorname{Re}=226,000 \& A o A=0^{\circ}, 30^{\circ}, 60^{\circ} \& 90^{\circ}\right)$

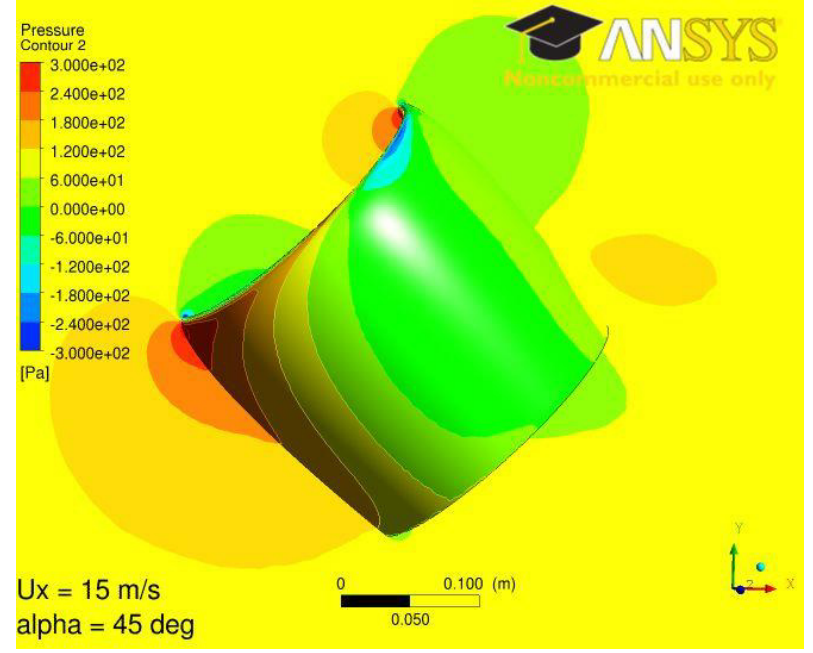

(a)

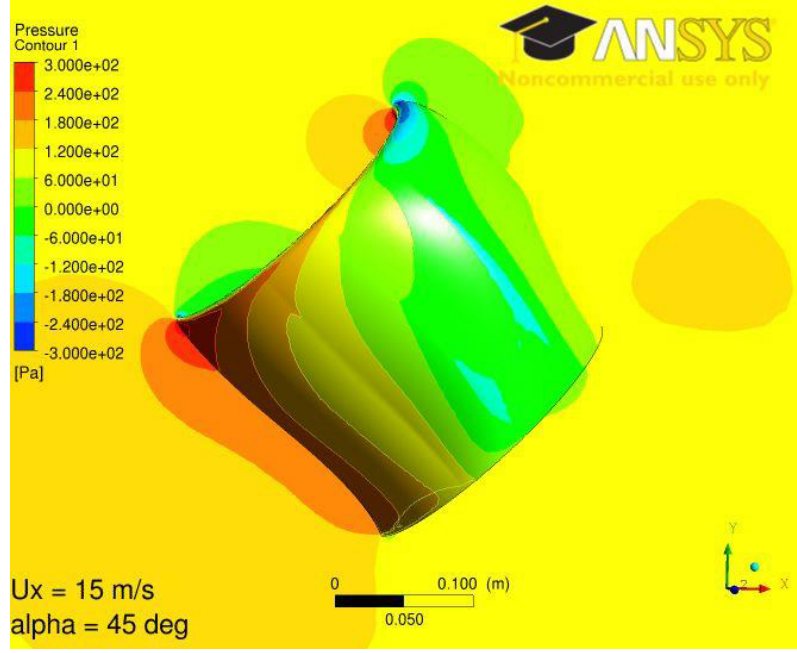

(b)

Figure 4-4: The contour of pressure on the half-body ducts (without fans) and plane of symmetry at $A o A=45^{\circ}$; (a) symmetrical duct, (b) asymmetrical duct

As a comparison between the two ducts, although the high curvature of the upper surface of the asymmetrical duct is in charge of higher lift generation in low AoAs, the specific shape of its lower surface is responsible for capturing more momentum from the passing flow in higher AoAs. This specific shape causes a reduction in the flow velocity close to the lower surface, which increases the local static pressure around that location in higher AoAs. This mechanism helps the asymmetrical duct to generate higher lift in the wide range of AoAs. 


\subsection{Experimental validation of the CFD results for ducts without fans}

To validate the CFD results, the actual models of symmetrical and asymmetrical ducts used in the CFD analysis are designed and produced using CATIA software and a 3D printer, as shown in Figure 4-5 and as explained in Appendix C. The specifications of the two ducts are listed in Table 3.3. The supporting frame was designed for attaching ducts to it, providing tilting motion for ducts through a gear system, and measuring AoA by a $360^{\circ}$ protractor. The additional mechanism for measuring the pitching moment was added to the supporting frame after measuring the lift and drag of the models, as shown in Figure 4-6. The ducts were installed in the large wind tunnel at Ryerson University with a $90 \times 90 \mathrm{~cm}$ test section (for more information see Appendix D).

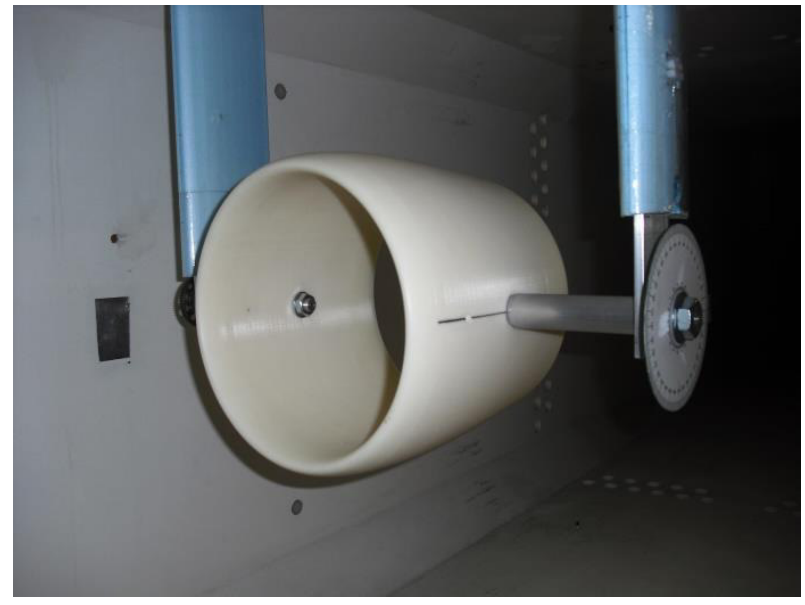

(a)

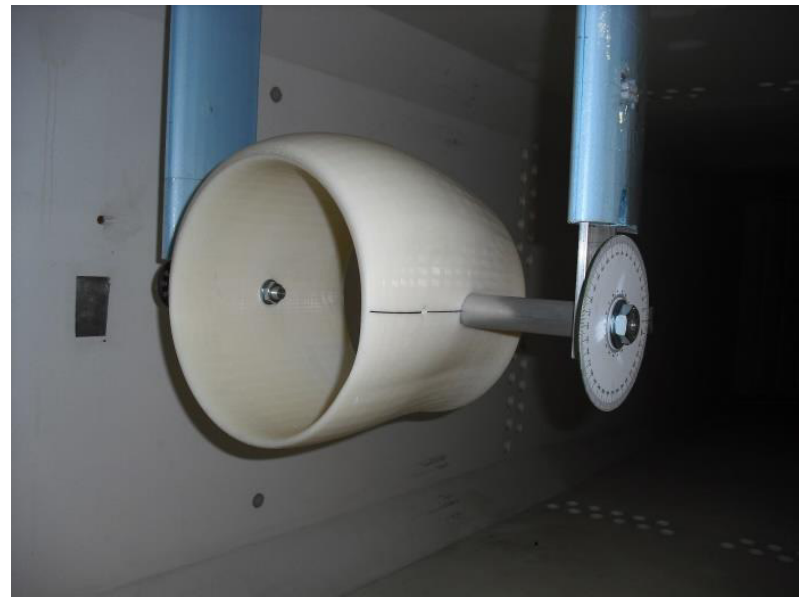

(b)

Figure 4-5: The symmetrical and asymmetrical ducts installed in the wind tunnel
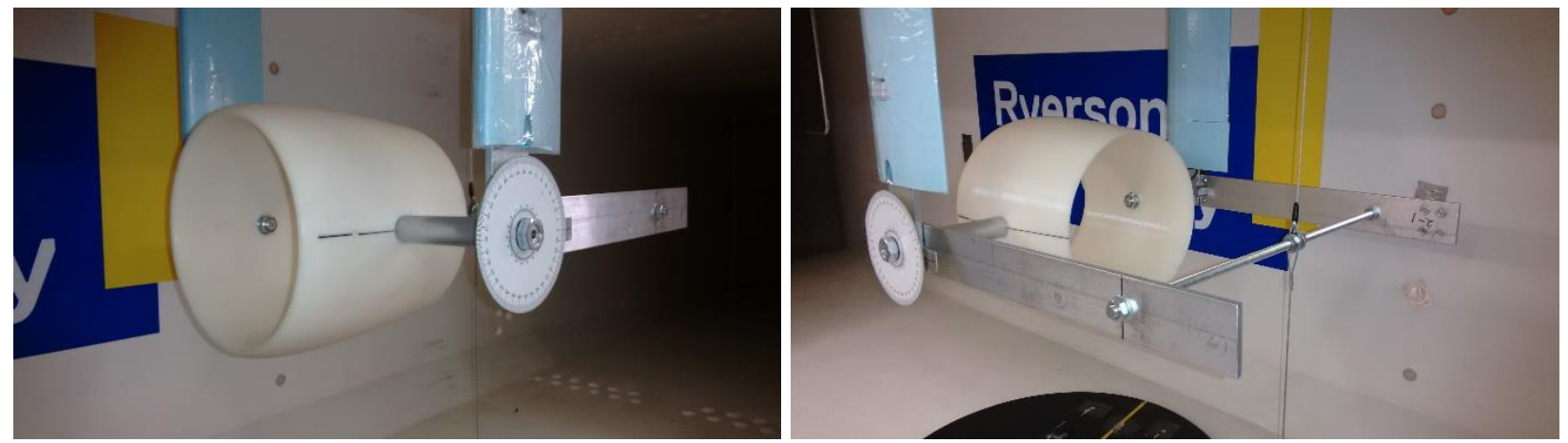

Figure 4-6: The mechanism used for measuring experimental pitching moment in the wind tunnel 
Flow velocity is set to $15 \mathrm{~m} / \mathrm{s}$ same as CFD simulations, which provides $\mathrm{Re}=226,000$ during wind tunnel tests. Each test was repeated three times to have accurate data and error analysis was done to validate the accuracy of measuring aerodynamic coefficients for both ducts without fans (see Appendix G). The experimental results are compared with the CFD results in the following figures. Figure 4-7 illustrates the comparison of the $C_{l}, C_{d}$, and $C_{m}$ from experiments with the CFD method for the symmetrical duct without a fan in $\mathrm{Re}=226,000$. Both experimental and CFD results show the same trends. The lift coefficient from the CFD simulation is very close to the experimental lift coefficient.

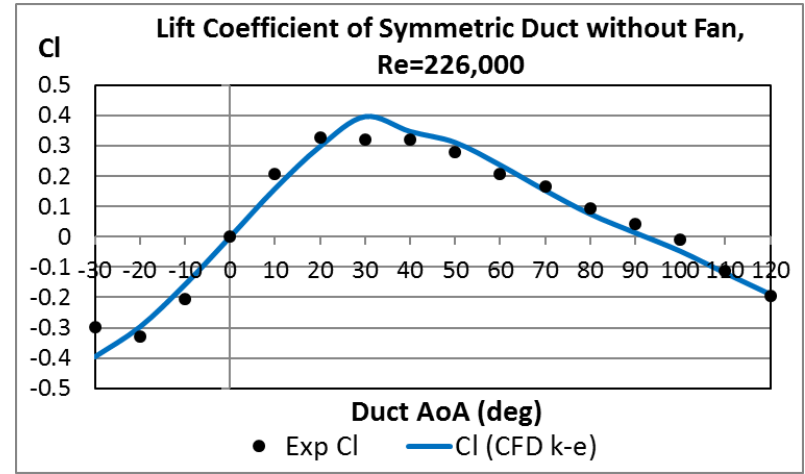

(a)

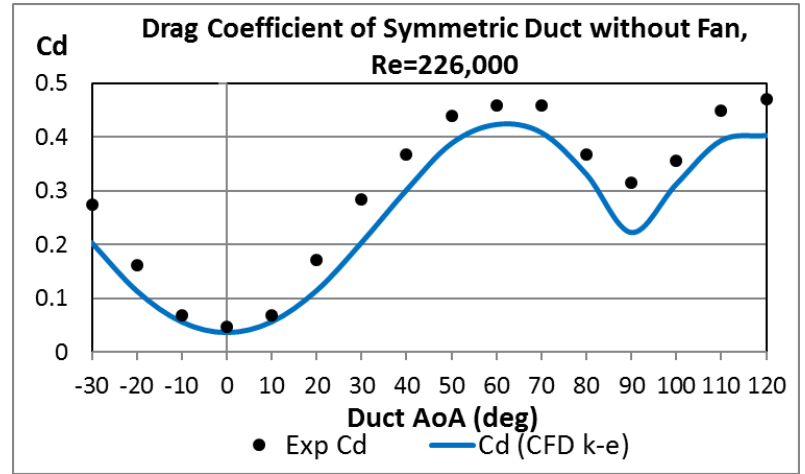

(b)

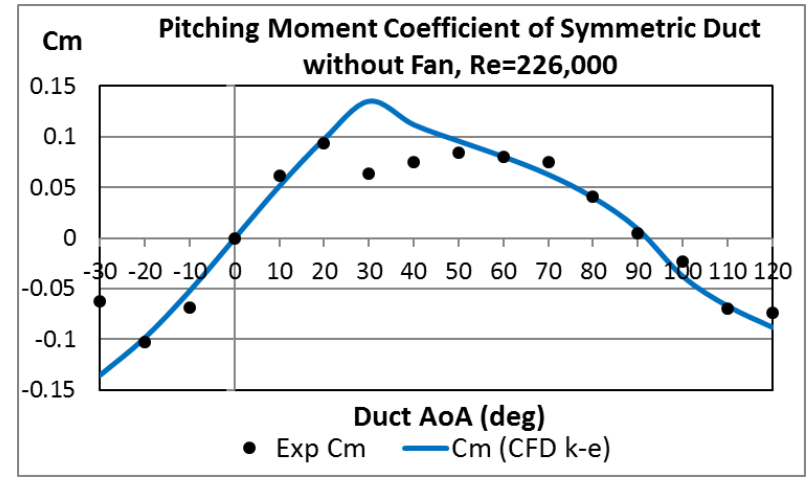

(c)

Figure 4-7: Comparison of (a) lift, (b) drag and (c) pitching moment coefficients from experiments with CFD for symmetrical duct without fan in $\mathrm{Re}=226,000$

As shown in Figure 4-7, the experimental $C_{m}$ in the range of $20^{\circ} \leq A o A \leq 50^{\circ}$ considerably deviates from the CFD result after the occurrence of stall at $\alpha=20^{\circ}$ and reduction in the $C_{l}$ for 
this range of AoAs. The CFD and experimental results converge again at $\alpha=50^{\circ}$. Also, the experimental $C_{d}$ is slightly greater than the CFD result at higher AoAs, this could be due to the skin friction of the actual models and test section wall effects. The reasons for these phenomena are discussed below.

Comparison between experimental $C_{l}, C_{d}$ and $C_{m}$ with the CFD results for the asymmetrical duct in $\mathrm{Re}=226,000$ (see Figure 4-8) show the same behavioural patterns as discussed for the symmetrical duct in Figure 4-7.

The stall conditions in the experiments occur at $\alpha=20^{\circ}$, whereas in the CFD modeling the stall occurs at a higher angle at $\alpha=30^{\circ}$ for both ducts. The occurrence of the stall at $\alpha=20^{\circ}$ and reduction in the $C_{l}$ in the range of $20^{\circ} \leq A o A \leq 50^{\circ}$ in the experiments causes the deviation between CFD and experimental values of $C_{m}$ for that range of AoAs. Although, the CFD and experimental $C_{l}$ converge again at $\alpha=50^{\circ}$, the existence of flow separation at the duct's lower side lips is the main reason for higher experimental $C_{m}$ in the higher AoAs. The disruption of the flow around the lower side lips of the asymmetrical duct causes greater differences between CFD and experimental values of $C_{m}$ which may continue up to $\alpha=90^{\circ}$, as shown in Figure 4-8c. This difference was reported by Fleming et al. as well, and the most likely explanation for the difference is flow separation around the windward duct lip was not captured in the CFD simulations [9].

Skin friction at the lower AoAs and the test section wall effects at higher AoAs are two reasons for the differences between the values of the experimental and the CFD drags. As described before, the CFD models consist of a 3D spherical domain with a radius of 10 characteristic duct chord lengths, and the test section of the wind tunnel has a cross section close to $4 \times 4$ characteristic duct chord length. This means the CFD models have a cross section 18 times larger than the wind tunnel test section. 


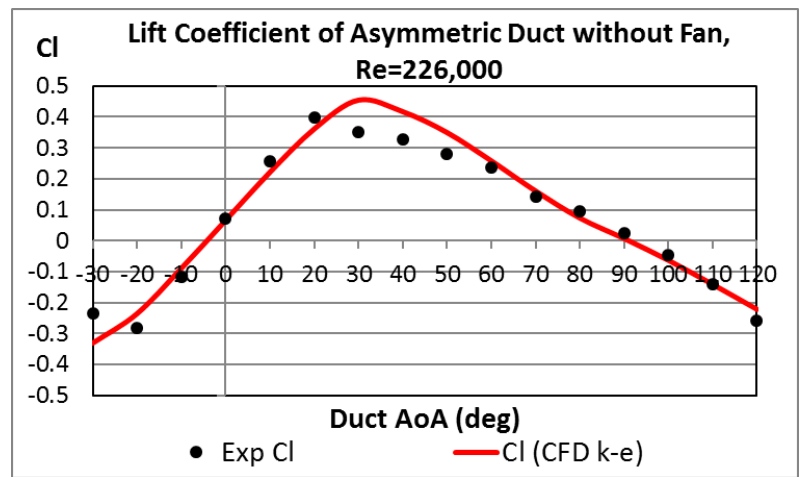

(a)

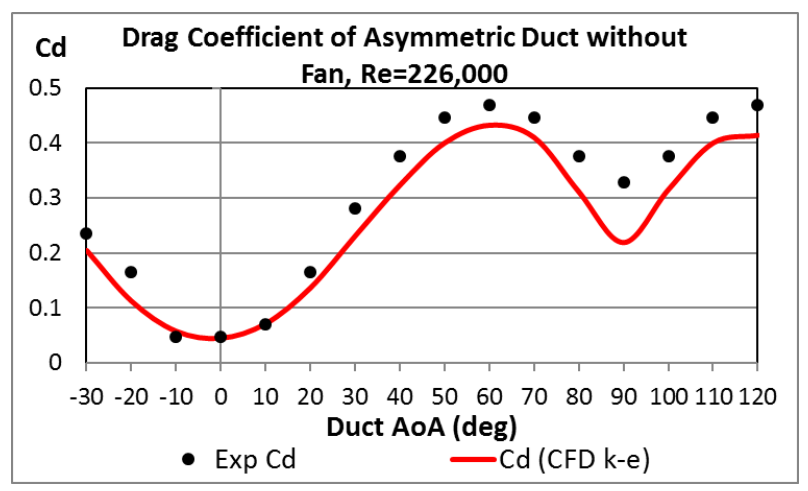

(b)

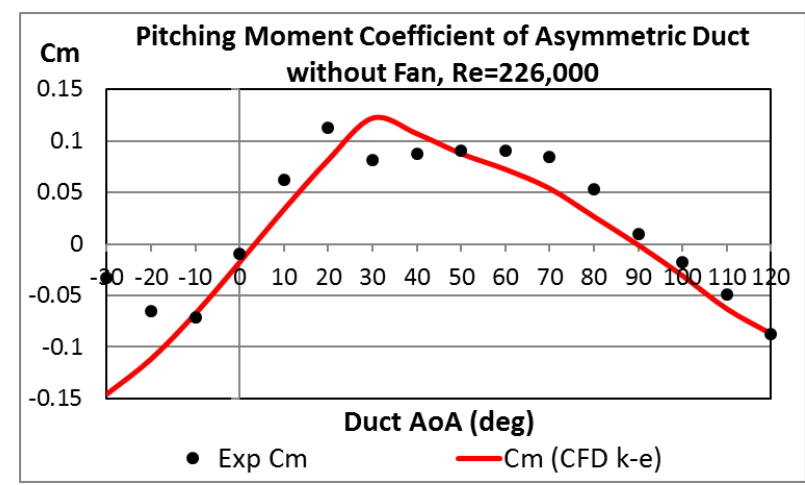

(c)

Figure 4-8: Comparison of (a) lift, (b) drag, and (c) pitching moment coefficients from experiments with CFD for asymmetrical duct without fan in $\mathrm{Re}=226,000$

By increasing AoA and blocking the inner section of the ducts in the range of $40^{\circ} \leq A o A \leq$ $120^{\circ}$, the flow passes through a smaller area in the test section. The result is a smaller area for passing flow around the ducts with higher velocity which creates higher drag forces in the experiments. As the tilting angle approaches $\alpha=90^{\circ}$, the lift coefficient of both ducts approaches zero $\left(C_{l_{\alpha=90^{\circ}}} \approx 0\right)$, which results in $L_{\alpha=90^{\circ}} \approx 0$ for both ducts.

The comparisons of experimental results for both ducts are shown in Figure 4-9. As illustrated in Figure 4-2 and predicted by the CFD modeling, the asymmetrical duct generates higher lift in the range of $-30^{\circ} \leq A o A \leq 40^{\circ}$ and drag forces of both ducts are similar for a wide range of AoAs. Due to a smaller radius of the duct lip at the lower surface of the asymmetrical duct, the 
flow separation has stronger effects. The incoming flow generates a slightly higher pitching moment for the asymmetrical duct than the symmetrical duct in the range of $20^{\circ} \leq A o A \leq 80^{\circ}$.

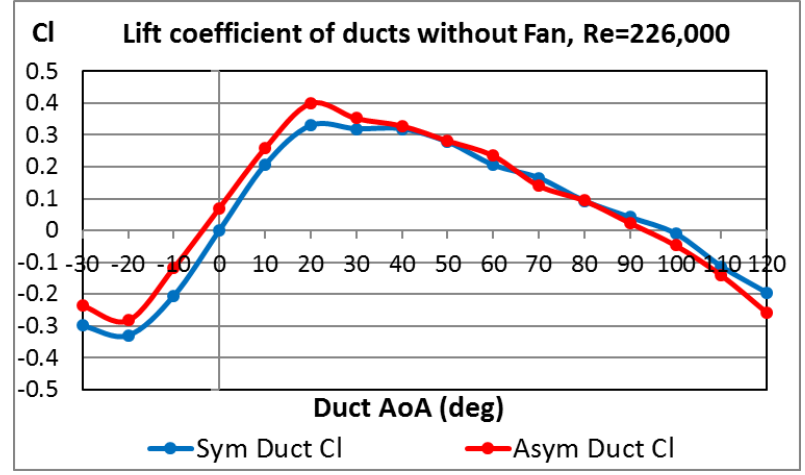

(a)

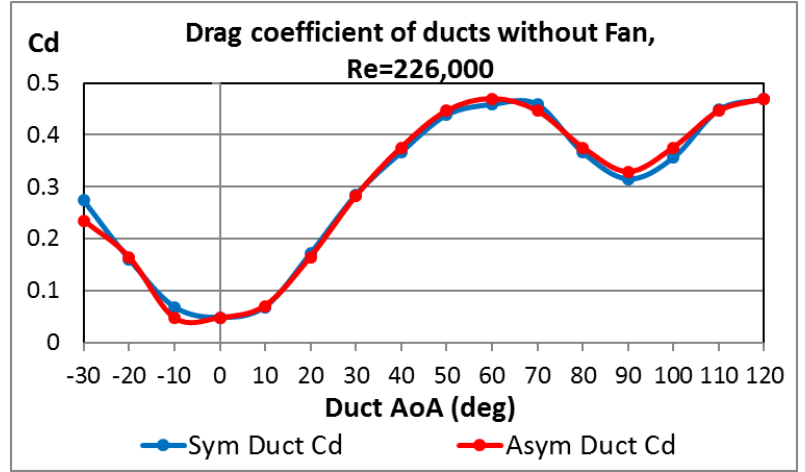

(b)

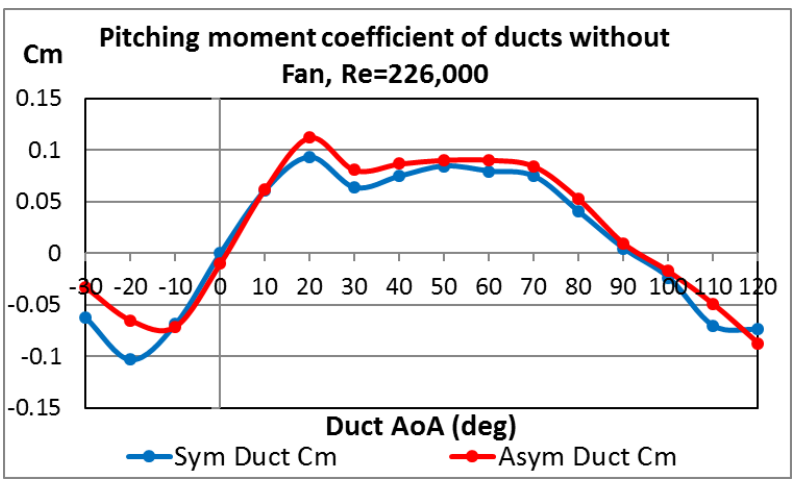

(c)

Figure 4-9: Comparing experimental (a) lift, (b) drag, and (c) pitching moment coefficients of symmetrical with asymmetrical ducts without fan, $\operatorname{Re}=226,000$

\subsection{Flow separation around the duct lips}

Comparing Figure 3-3b with Figure 3-4d shows that the asymmetrical duct has a larger radius at the upper side and smaller radius at the lower-side of the leading edges compared with the symmetrical duct. By increasing AoA in the transition from cruise mode to hover, the flow separation starts from the ducts' lower-side lips at $A o A=20^{\circ}$. Due to smaller radius of the asymmetrical duct's lower-side lip, the flow separation has stronger effects (such as reduction in the lift) compared to the symmetrical duct in the range of $20^{\circ} \leq A o A \leq 40^{\circ}$, as shown in Figure 
4-9a. The lift force is equal for both ducts in the range of $40^{\circ} \leq A o A \leq 90^{\circ}$. Comparison between drag forces does not show meaningful effects of the duct lip flow separation in the range of $0^{\circ} \leq$ $A o A \leq 120^{\circ}$, as illustrated in Figure 4-9b. As discussed before, the main objective of this part of the research was to compare the aerodynamic forces and pitching moments of two different geometries for the external shape of the duct. This means that the effects of the fan/rotor presence (such as thrust and ram drag variations) are not discussed in this section. Flow separation around the duct lips causes minor instability on the lift generation in the absence of fan/rotor. The flow separation combined with ram drag and thrust variations will have larger effects on the pressure distribution and aerodynamic forces for ducted fans, as will be discussed in following sections.

\subsection{The CFD simulations for symmetrical and asymmetrical ducted fans}

In this section, two meshes for symmetrical and asymmetrical ducted fans (see Table 3.2) are designed as shown in Figure 4-10.

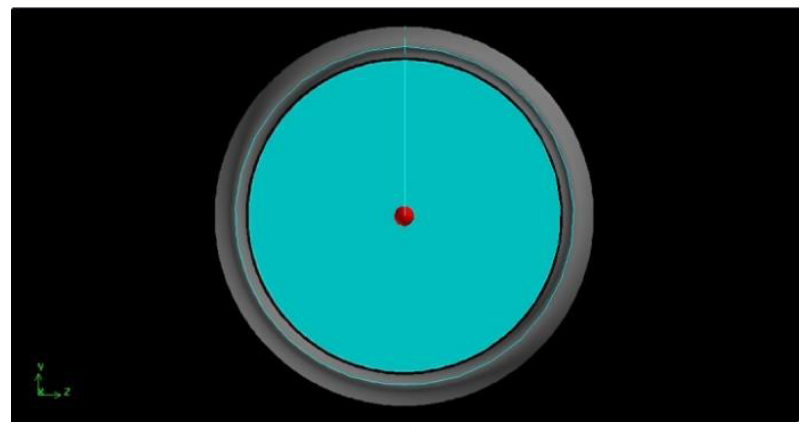

(a)

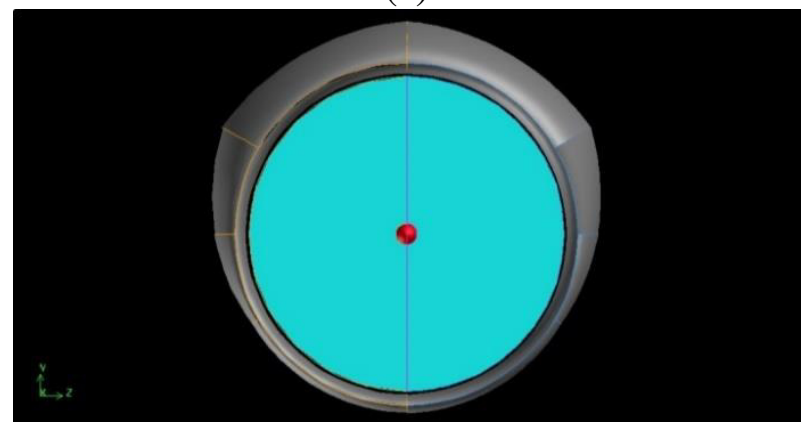

(c)

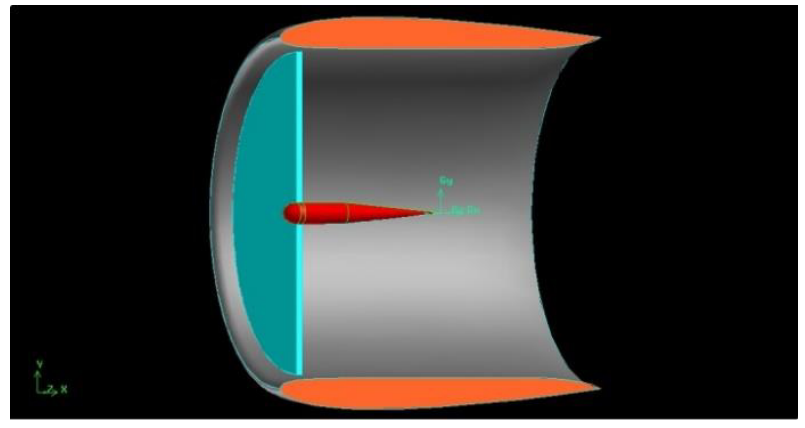

(b)

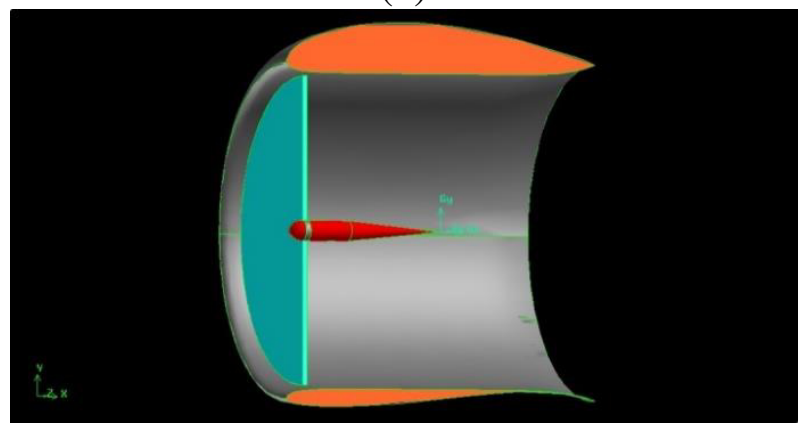

(d)

Figure 4-10: Two ducted fans; symmetrical duct: (a) front view, (b) cross section in xy plane and asymmetrical duct: (c) front view, (d) cross section in $x y$ plane 
These models were simulated in stationary conditions and the ANSYS-Fluent software final setup for each ducted fan is listed in Table 4.1.

Table 4.1: Final setup for the ANSYS-Fluent software

\begin{tabular}{|l|l|}
\hline \multicolumn{1}{|c|}{ Items } & \multicolumn{1}{c|}{ Entered values } \\
\hline Freestream velocity and duct's AoA range & $U=15 \mathrm{~m} / \mathrm{s},-30^{\circ} \leq \mathrm{AoA} \leq 120^{\circ}$ \\
\hline Inlet flow velocity & From Table 3.6 based on AoA \\
\hline Turbulence modeling & $\mathrm{k}-\varepsilon$ Re-Normalisation Group \\
\hline Max. No. of iterations for running software in each AoA & 800 \\
\hline
\end{tabular}

The resultant forces and pitching moments were calculated using $V_{\text {ind }}$ from Table 4.1 and equations (3.25) - (3.29) and (3.34). The results of these calculations are shown in Figure 4-11, and the resultant forces and pitching moments are compared for two ducted fans. It can be seen that both ducted fans have the same trends for all resultant forces and pitching moments. In addition, $\Sigma F_{x}$ and $\Sigma M_{o}$ of the symmetrical ducted fan are very close to those for the asymmetrical ducted fan (see Figure 4-11b and Figure 4-9c). It was shown in Figure 4-2a that the asymmetrical body shape can generate additional lift compared to the symmetrical body shape. This result is repeated here again for ducted fans. The $\Sigma F_{y}$ diagram (see Figure 4-11a) proves that the asymmetrical ducted fan has higher generated lift than the symmetrical ducted fan. In these simulations, the range of generated lift by the symmetrical and asymmetrical ducts are $-3.73 \mathrm{~N} \leq$ $L_{\text {Sym duct }} \leq 4.28 N$ and $-2.38 N \leq L_{\text {Asym duct }} \leq 5.48 N$, respectively. The difference between two lift forces is noticeable and the asymmetrical ducted fan has generated more lift than the symmetrical ducted fan for a wide range of tilting angle $\left(-30^{\circ} \leq A o A \leq 70^{\circ}\right)$. This additional lift (around $1.87 \mathrm{~N}$ in cruise mode with $A o A=0^{\circ}$ ) for the asymmetrical ducted fan can help in designing a smaller wing for a VTOL UAV, which would then reduce overall drag and save more energy to increase the range in the cruise mode. As illustrated in Figure 4-11b, $\Sigma F_{x}=0$ is obtained for both ducted fans in the cruise mode $\left(A o A=0^{\circ}\right)$ using different delivered powers to the 
propellers as discussed in the previous section. The most important parameters in the cruise mode for both ducted fans are listed in Table 4.2.

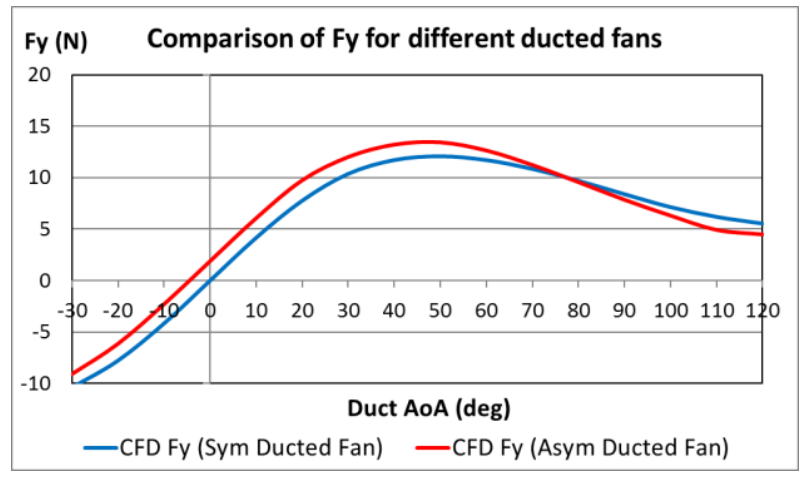

(a)

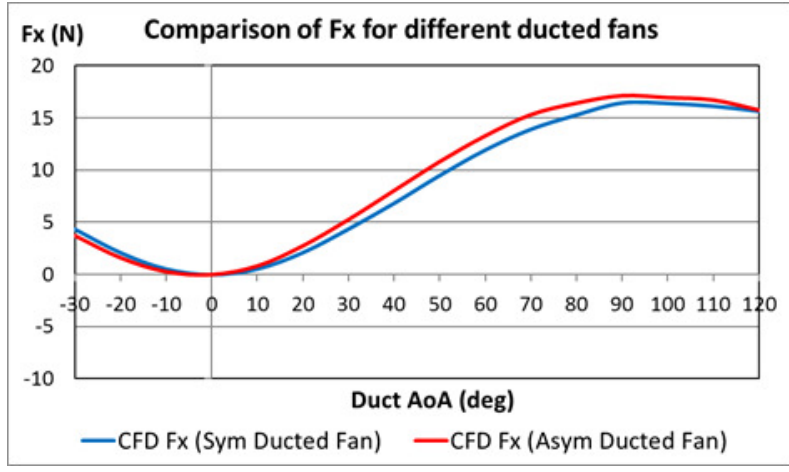

(b)

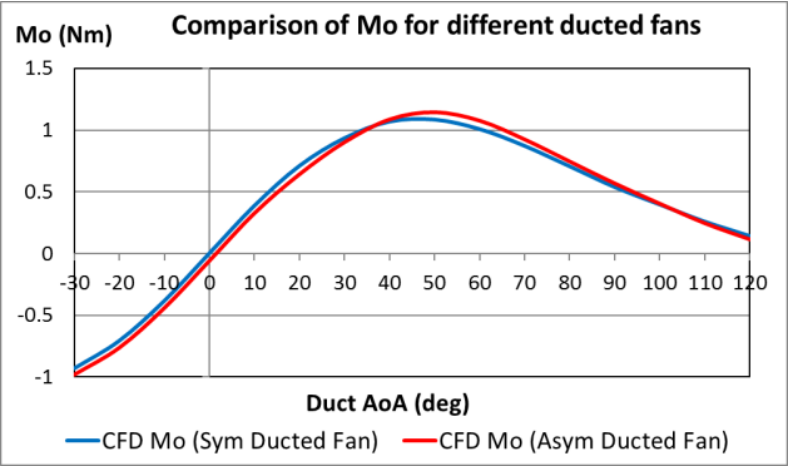

(c)

Figure 4-11: The variation of aerodynamic forces and pitching moments for symmetrical and asymmetrical ducted fans in $\operatorname{Re}=226,000$; (a) $F_{y}$, (b) $F_{x}$, and (c) $M_{o}$.

Table 4.2: Important parameters for both ducted fans in cruise mode $\left(U=15 \mathrm{~m} / \mathrm{s} \& A o A=0^{\circ}\right)$

\begin{tabular}{|c|c|c|c|c|c|c|}
\hline Type of ducted fan & $\begin{array}{c}\text { Power } \\
(\text { watt })\end{array}$ & $\begin{array}{c}V_{\text {ind }} \\
(\mathrm{m} / \mathrm{s})\end{array}$ & $\begin{array}{c}\text { Thrust } \\
(\mathrm{N})\end{array}$ & $\begin{array}{c}D_{\text {frame }} \\
(\mathrm{N})\end{array}$ & $\begin{array}{c}D_{\text {duct }} \\
(\mathrm{N})\end{array}$ & $\begin{array}{c}L_{\text {duct }} \\
(\mathrm{N})\end{array}$ \\
\hline Symmetrical & 61.908 & 21.25 & 2.913 & 2.4606 & 0.452 & 0 \\
\hline Asymmetrical & 65.154 & 21.40 & 3.045 & 2.4606 & 0.584 & 1.856 \\
\hline
\end{tabular}

As listed in Table 4.2, the asymmetrical ducted fan uses 5\% more power than the symmetrical ducted fan to generate $4.3 \%$ more thrust. Although the asymmetrical ducted fan generated higher drag in this simulation, it could generate significant additional lift $(1.856 \mathrm{~N})$, which can help in designing a smaller wing and reducing overall drag of the VTOL UAV. 
As illustrated in Figure 4-11c, both ducted fans have almost the same overall pitching moments. The ram drag has a noticeable role in producing pitching moment in the transition. To reduce pitching moment due to ram drag during transition from cruise mode to hover, it is necessary to move the tilting axis location from the middle of the duct closer to the rotor plane (or somewhere between the rotor plane and the aerodynamic centre of the ducts).

The contours of pressure around the ducted fans are shown in Figure 4-12 for a wide range of AoAs. The contours of pressure on the plane of symmetry around two ducted fans for $A o A=45^{\circ}$ are also presented in Figure 4-13. As it was explained for the asymmetrical duct without a fan, the high curvature of the upper surface is in charge of the higher lift generation in the low AoAs. In addition, the specific shape of the lower surface is responsible for capturing more momentum from the passing flow in the higher AoAs. This specific shape causes a reduction in the flow velocity close to the lower surface, which increases the local static pressure around that location in higher AoAs. As a result, this mechanism helps the asymmetrical duct to generate the higher lift in the wide range of AoAs.
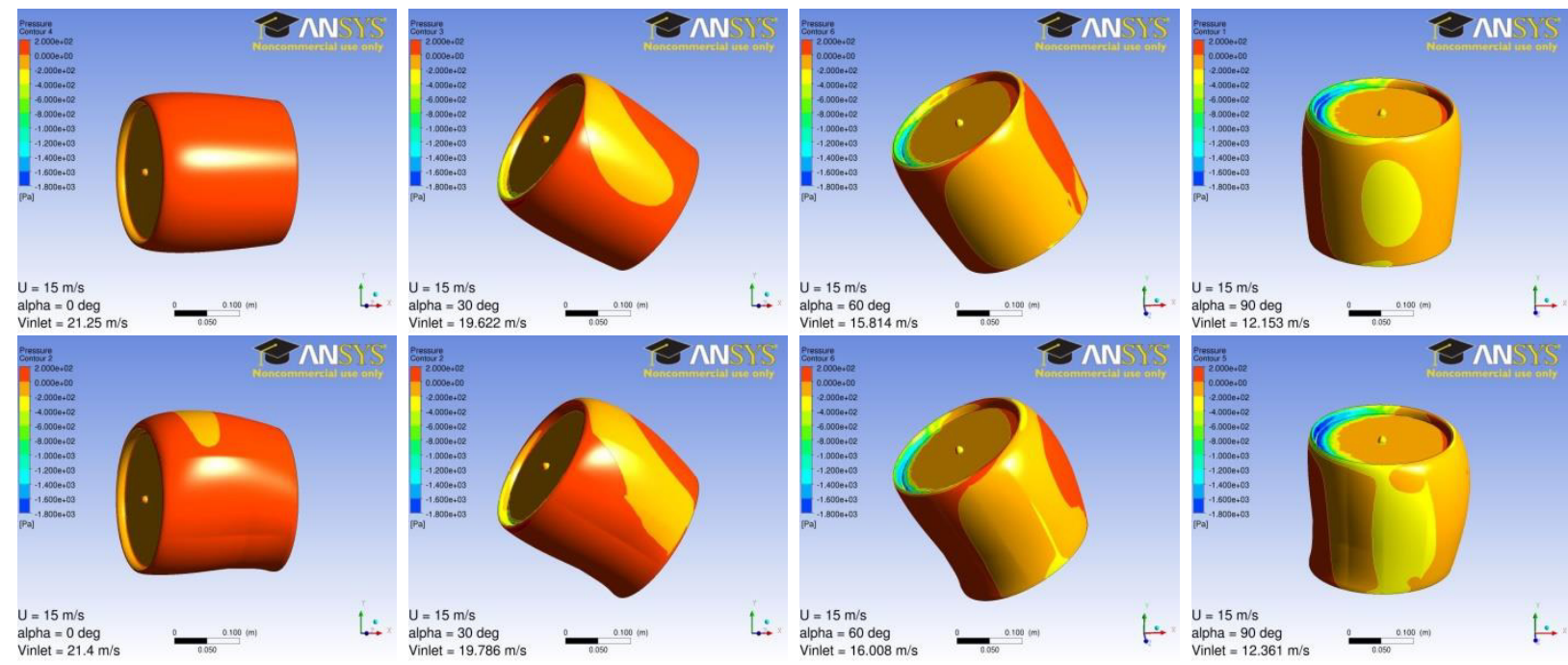

Figure 4-12: The contour of pressure around symmetrical ducted fan (top) and asymmetrical ducted fan (bottom) in steady flow $\left(U=15 \mathrm{~m} / \mathrm{s} \& A o A=0^{\circ}, 30^{\circ}, 60^{\circ} \$ 90^{\circ}\right)$ 


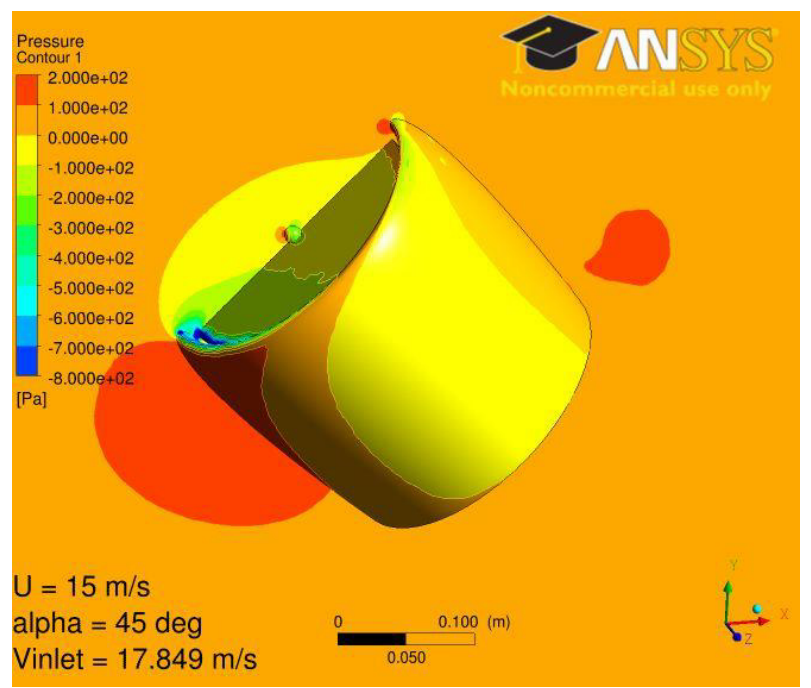

(a)

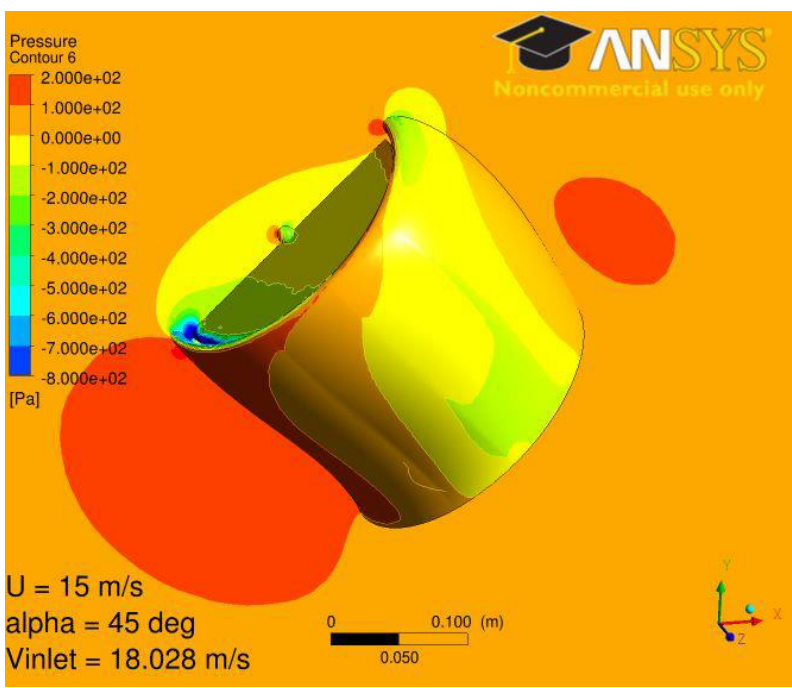

(b)

Figure 4-13: The contour of pressure on the half-body ducted fans and plane of symmetry at $A o A=45^{\circ}$; (a) symmetrical ducted fan, (b) asymmetrical ducted fan

\subsection{Aerodynamic coefficients for ducted fans by CFD modeling}

Figure 4-11 shows the summation of the aerodynamics forces, thrust and ram drag and their related pitching moments. By eliminating thrust as well as ram drag and its associated pitching moment, the net aerodynamic forces and pitching moment coefficients $\left(C_{l}, C_{d}\right.$, and $\left.C_{m}\right)$ can be calculated using equations (3.27) - (3.29). The aerodynamic coefficients for two ducted fans under the influence of active fans are shown in Figure 4-14. The free stream velocity is set to $U=15 \mathrm{~m} / \mathrm{s}$ to get $\operatorname{Re}=226,000$. Table 3.6 is used to set the inlet velocity of the flow into the ducted fans $\left(V_{\text {ind }}\right)$ based on the related AoA.

As expected and can be seen in Figure 4-14a, the $C_{l}$ for asymmetrical ducted fan is larger than that of the symmetrical ducted fan for a wide range of AoAs $\left(-30^{\circ} \leq A o A \leq 75^{\circ}\right)$. The $C_{d}$ of both ducts are very similar in cruise mode $\left(A o A \approx 0^{\circ}\right)$ and $C_{m}$ does not show significant difference for the whole range of AoAs. 


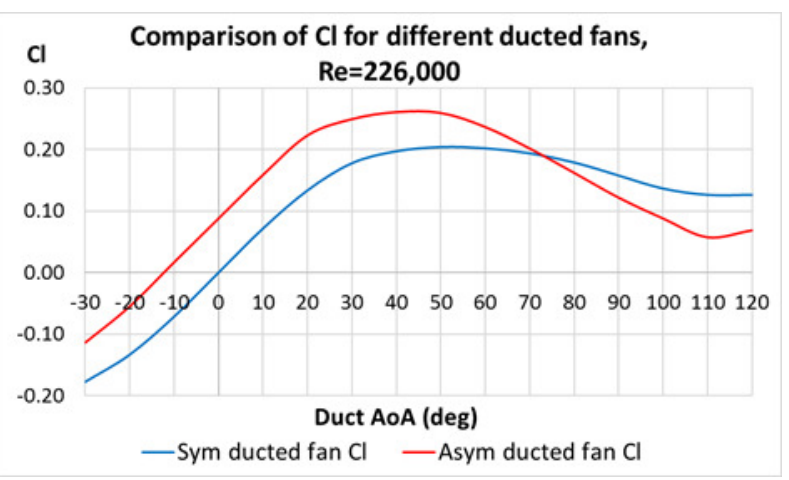

(a)

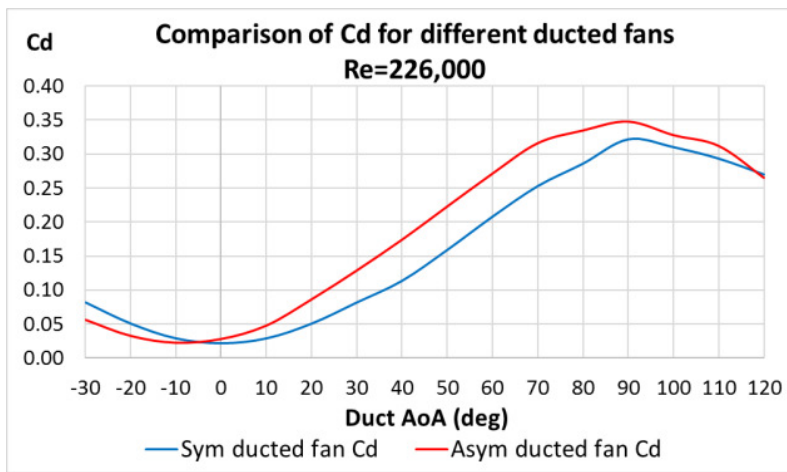

(b)

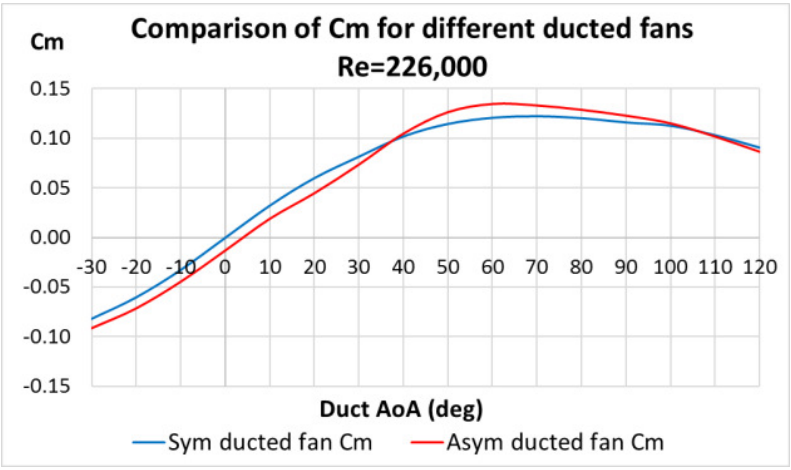

(c)

Figure 4-14: The variation of aerodynamic coefficients for symmetrical and asymmetrical ducted fans under the influence of active fans in different AoA (a) $C_{l}$, (b) $C_{d}$, and (c) $C_{m}, \operatorname{Re}=226,000$.

\subsection{Experimental validation of the CFD results for ducted fans}

To validate the CFD results, both symmetrical and asymmetrical ducts are equipped with electro motors and propellers, as shown in Figure 4-15 and tested in the large wind tunnel at Ryerson University. The propeller specification is listed in Table 3.3. The wind tunnel flow velocity is set to $15 \mathrm{~m} / \mathrm{s}$ to compare the experimental results with previous CFD simulations. Each test was repeated three times to have accurate data. The error analysis was done to validate the accuracy of measuring aerodynamic coefficients for both ducted fans (see Appendix G). 


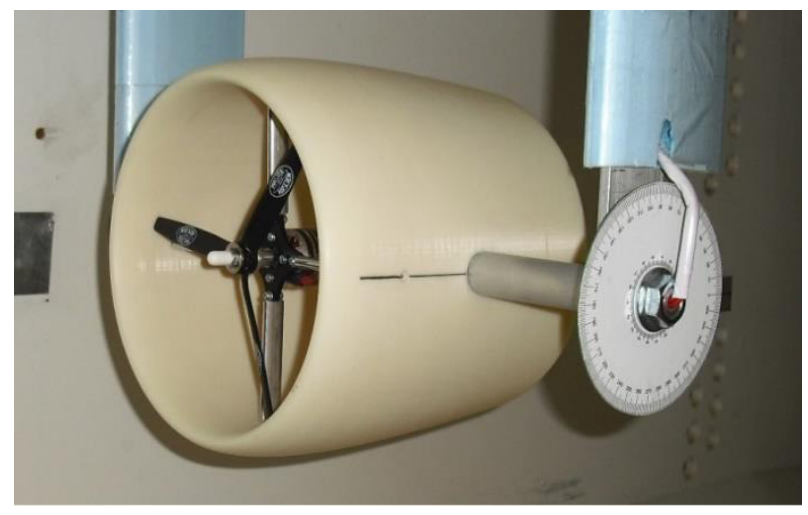

(a)

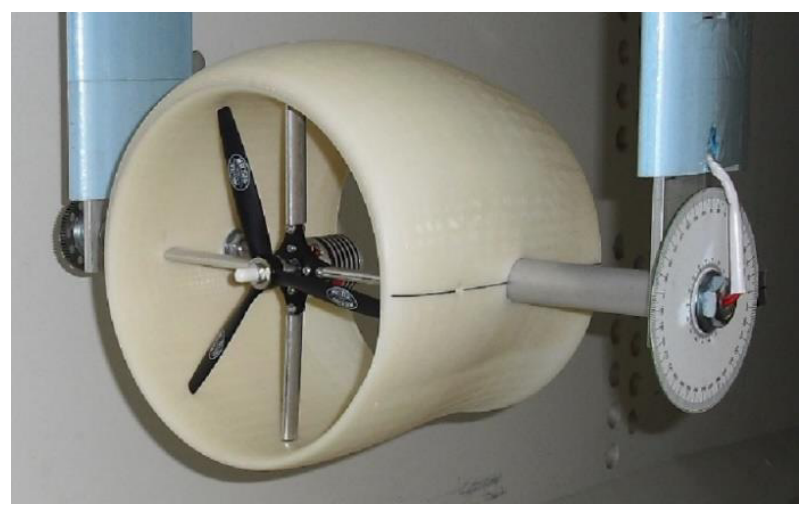

(b)

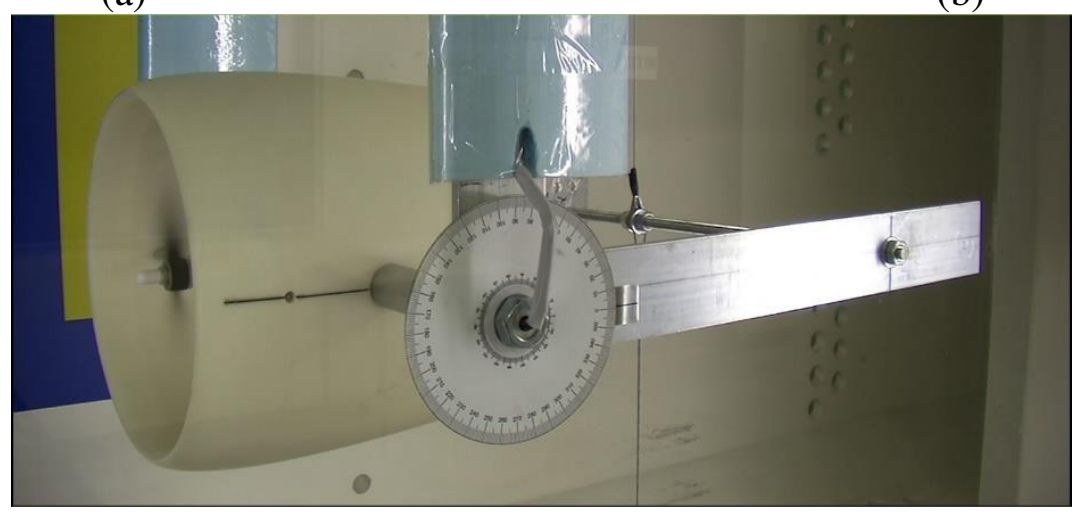

(c)

Figure 4-15: The symmetrical and asymmetrical ducted fans installed in the wind tunnel and the mechanism used for measuring experimental pitching moment in the wind tunnel

The gross horizontal and vertical forces and pitching moments $\left(\Sigma F_{x}, \Sigma F_{y}\right.$, and $\left.\Sigma M_{o}\right)$ are recorded by the wind tunnel measuring system for whole combinations of ducted fans attached to the supporting frame. Next, the supporting frame's drag $\left(D_{\text {frame }}=2.4606 \mathrm{~N}\right)$, and pitching moment due to the ducted fans' weights in different AoAs, were subtracted from recorded gross forces and pitching moments to obtain the net forces and pitching moments acting on the ducted fans by using equations (3.25)-(3.29) and (3.34). The measured pitching moments due to the weight of ducts with/without fans in different AoAs are presented in Appendix F.

The CFD simulations provided net aerodynamic forces and pitching moments based on the pressure distribution around the ducted fans (without the effects of the ducts' weight and supporting frames). Thrust and ram drag were then calculated based on the inlet flow velocity to 
the rotor plane (as listed in Table 3.6 for both ducts based on the ducts' AoAs). Next, the vertical and horizontal components of calculated thrust and ram drag and generated pitching moments were added to simulated aerodynamic forces and pitching moments to obtain the final CFD results.

The comparison between experimental and CFD results are shown for different ducted fans in the following figures. Figure 4-16 illustrates the comparison of $\Sigma F_{y}, \Sigma F_{x}$, and $\Sigma M_{o}$ from experiments with CFD method for symmetrical ducted fan.

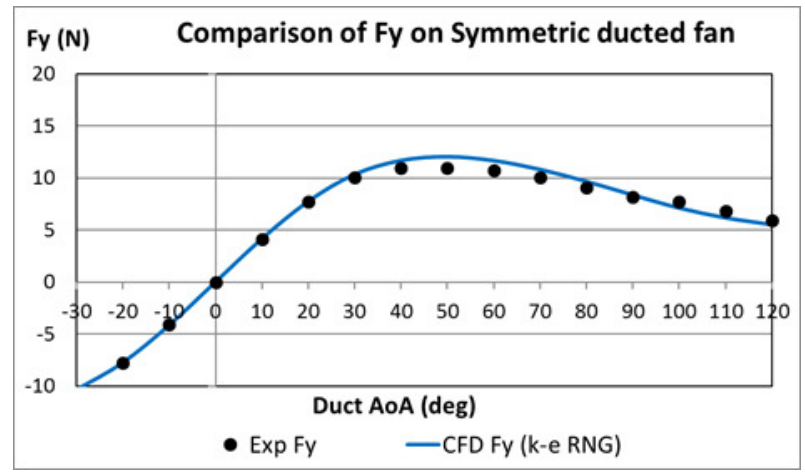

(a)

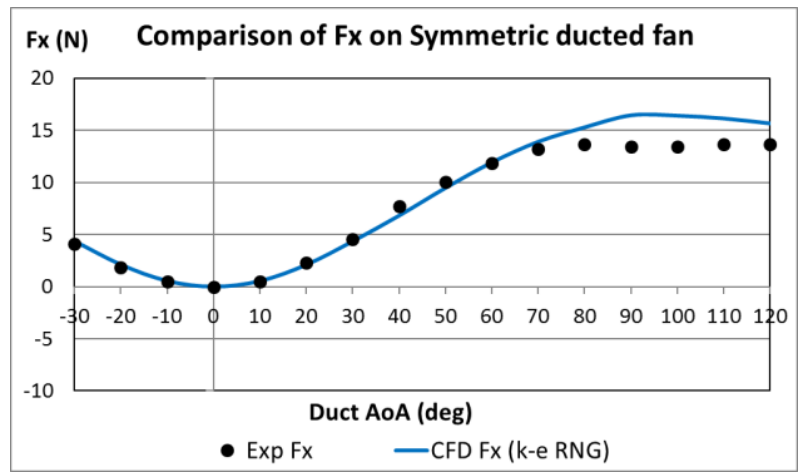

(b)

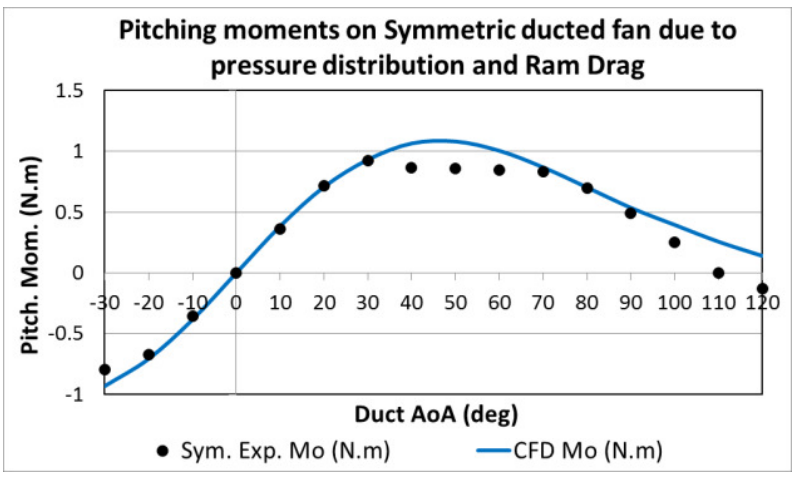

(c)

Figure 4-16: The variation of resultant forces and pitching moments for symmetrical ducted fan in $\operatorname{Re}=226,000$; (a) $F_{y}$, (b) $F_{x}$, and (c) $M_{o}$.

Both experimental and CFD results show the same trends. The calculated vertical force $\left(\Sigma F_{y}\right)$ from CFD simulation is very close to the experimental $\Sigma F_{y}$. The calculated resultant horizontal force $\left(\Sigma F_{x}\right)$ from CFD modeling at higher AoAs $\left(70^{\circ} \leq A o A \leq 120^{\circ}\right)$ is slightly higher than that in the experiment. In reality and at higher AoAs, the separated flow around the windward duct lip has strong effects, which result in the distortion of the velocity magnitude at the duct's inlet [11]. 
One effect could be reduction in freestream velocity and related ram drag (see equation (3.25)) close to the rotor plane, which results in reduction in the resultant horizontal force $\left(\Sigma F_{x}\right)$, but not on the resultant vertical force $\left(\Sigma F_{y}\right)$.

This phenomenon can be explained by substituting the values of ram drag and thrust from equations (3.25) and (3.34) into equation (3.27) and (3.28). The final equations for resultant horizontal and vertical forces are obtained as follows:

$\Sigma F_{x}=D_{\text {duct }}+D_{\text {frame }}+\rho A V_{\text {ind }} U-\rho A V_{\text {ind }}{ }^{2} \cos \alpha / a_{w}$

$\Sigma F_{y}=L_{d u c t}-W+\rho A V_{\text {ind }}^{2} \sin \alpha / a_{w}$

Comparison between equations (4.1) and (4.2) shows that reduction in the freestream velocity $(U)$ at the duct's inlet area causes the reduction in the resultant horizontal force $\left(\Sigma F_{x}\right)$, due to the existence of $\rho A V_{\text {ind }} U$ term in equation (4.1) and it has less effects on the resultant vertical force $\left(\Sigma F_{y}\right)$. All these happen because flow separation around the windward duct lip was not captured in CFD simulations [9]. The resultant pitching moments from the experiment and CFD modeling are shown in Figure 4-16c. Although two regions of $40^{\circ} \leq A o A \leq 60^{\circ}$ and $100^{\circ} \leq A o A$ are showing small differences between two methods, both methods give the same results for the rest of the duct's AoAs. This could be due to stall condition when $40^{\circ} \leq A o A \leq 60^{\circ}$ and flow separation in higher AoAs.

Figure 4-17 shows the comparison of $\Sigma F_{y}, \Sigma F_{x}$, and $\Sigma M_{o}$ from experiments with CFD method for the asymmetrical ducted fan. The explanations of the results are the same as the symmetrical ducted fan. Due to smaller radius of the asymmetrical duct lower-side lip, the flow separation happens sooner in the asymmetrical ducted fan $\left(\alpha \approx 50^{\circ}\right)$ during the experiment and has larger effects on the pitching moment. 


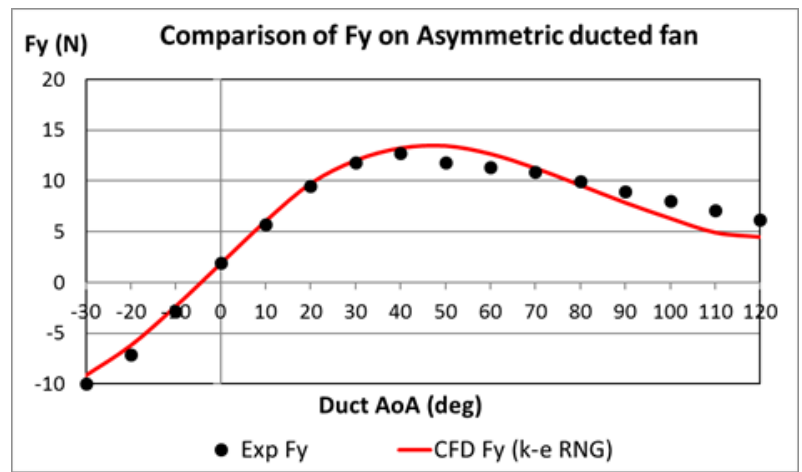

(a)

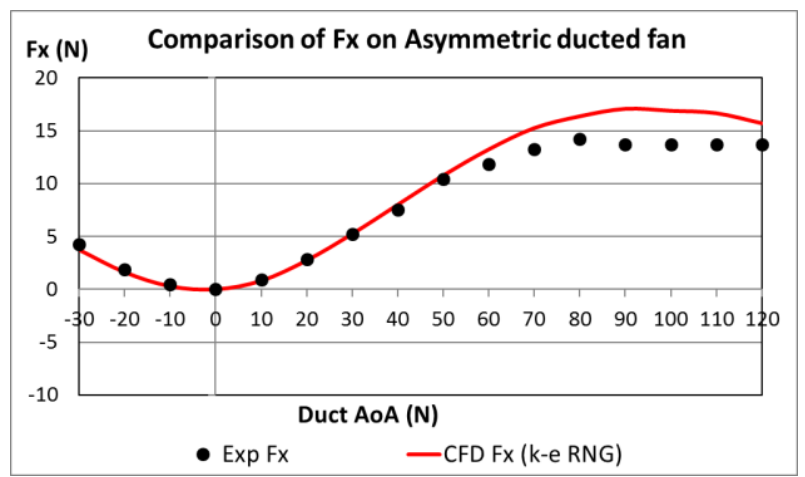

(b)

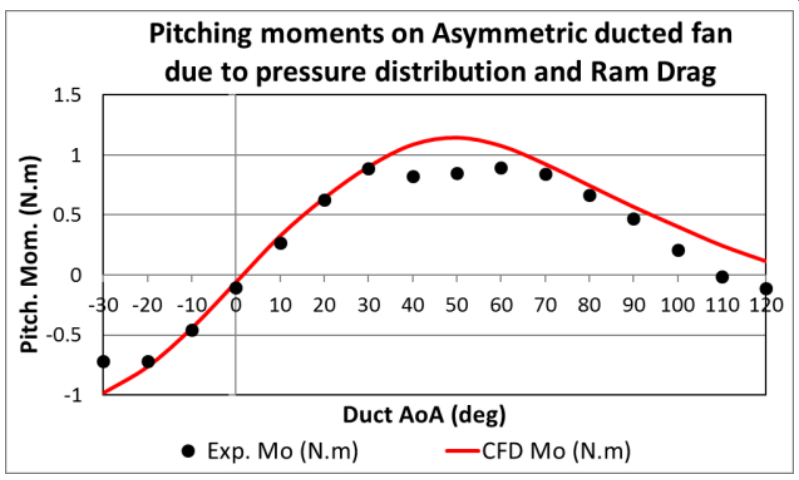

(c)

Figure 4-17: The variation of resultant forces and pitching moments for asymmetrical ducted fan in $\operatorname{Re}=226,000$; (a) $F_{y}$, (b) $F_{x}$, and (c) $M_{o}$.

The comparisons of experimental results for the symmetric and asymmetric ducted fans are shown in Figure 4-18. As illustrated in Figure 4-11 and predicted by CFD modeling, the asymmetrical ducted fan generates higher $\Sigma F_{y}$ in the range of $-30^{\circ} \leq A o A \leq 50^{\circ}$ and $\Sigma F_{x}$ for both ducted fans are similar for wide range of AoAs. Furthermore, the resultant vertical force $\left(\Sigma F_{y}\right)$ for the asymmetrical ducted fan in cruise mode is $\Sigma F_{y_{\alpha=0^{\circ}}}=1.893 \mathrm{~N}$, which is close to the value predicted by CFD modeling (1.856 $N$ with $2 \%$ error). 


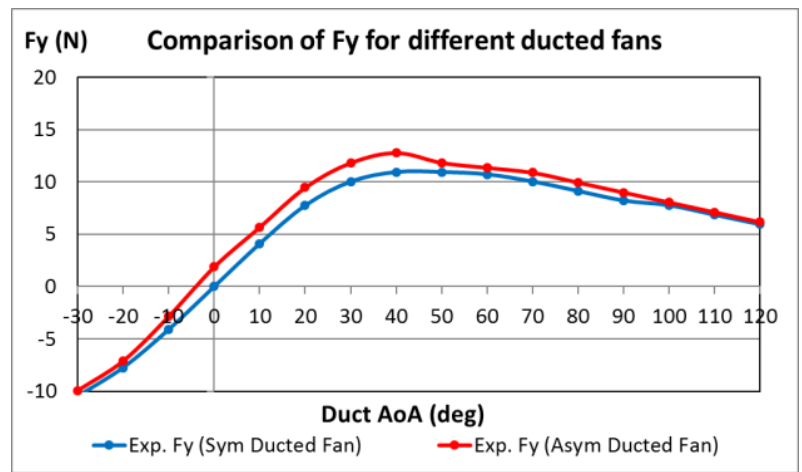

(a)

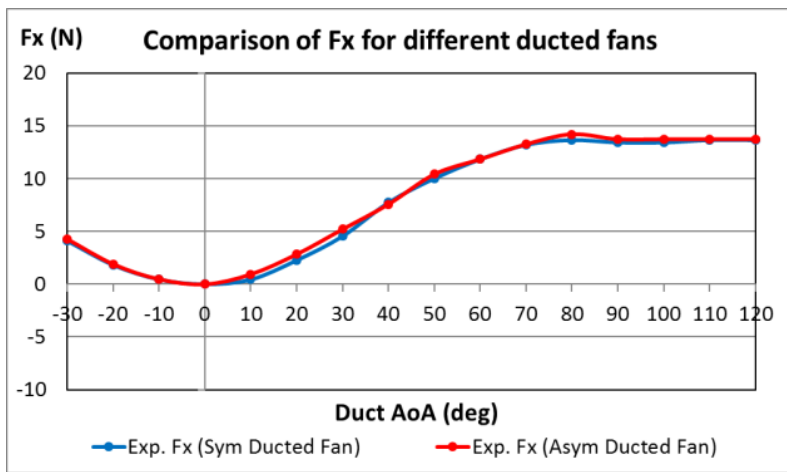

(b)

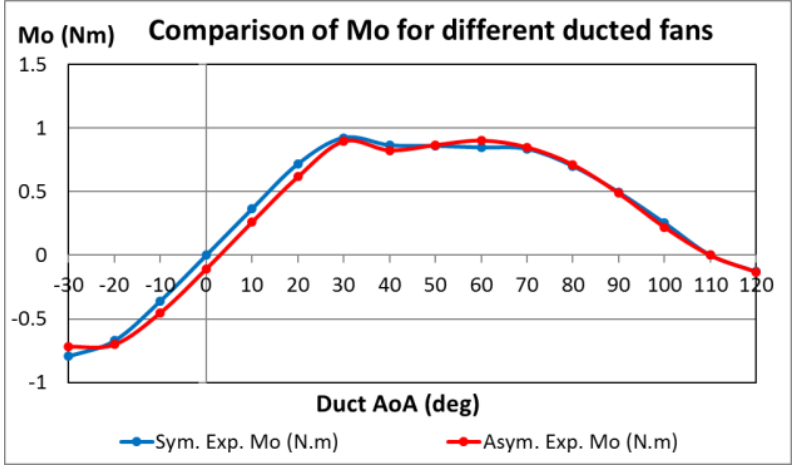

(c)

Figure 4-18: Comparing experimental resultant forces and pitching moments of symmetrical and asymmetrical ducted fans, $\operatorname{Re}=226,000$; (a) $F_{y}$, (b) $F_{x}$, and (c) $M_{o}$.

\subsection{Aerodynamic coefficients for ducted fans from wind tunnel experiments}

The summation of the aerodynamics forces, thrust and ram drag and their related pitching moments of two ducted fans are illustrated in Figure 4-18. By eliminating thrust, ram drag and its associated pitching moment, the net aerodynamic forces and pitching moment coefficients $\left(C_{l}, C_{d}\right.$, and $\left.C_{m}\right)$ can be calculated using equations (3.27) - (3.29). The aerodynamic coefficients for two ducted fans under the influence of active fans are shown in Figure 4-19. The wind tunnel free stream velocity was set to $U=15 \mathrm{~m} / \mathrm{s}$ to get $\mathrm{Re}=226,000$. As can be seen, trends for aerodynamic coefficients for both ducted fans are the same, especially for the drag and pitching moment coefficients (Figure 4-19a and b). As expected and predicted by CFD modeling, the lift coefficient of the asymmetrical ducted fan is higher than that for the symmetrical ducted fan. 


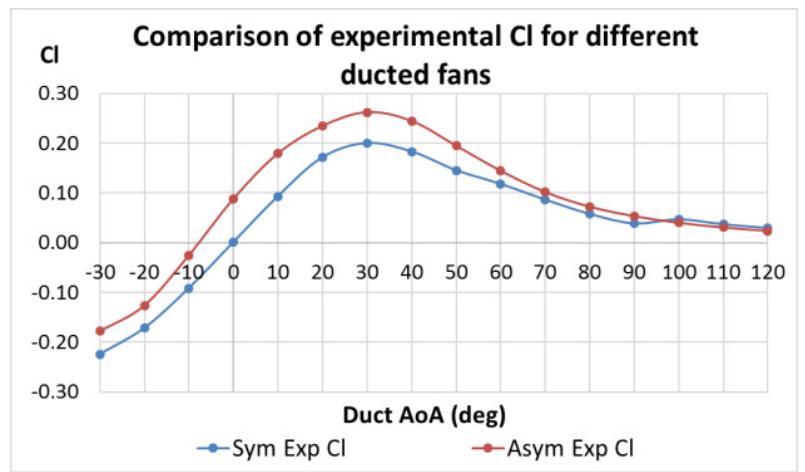

(a)

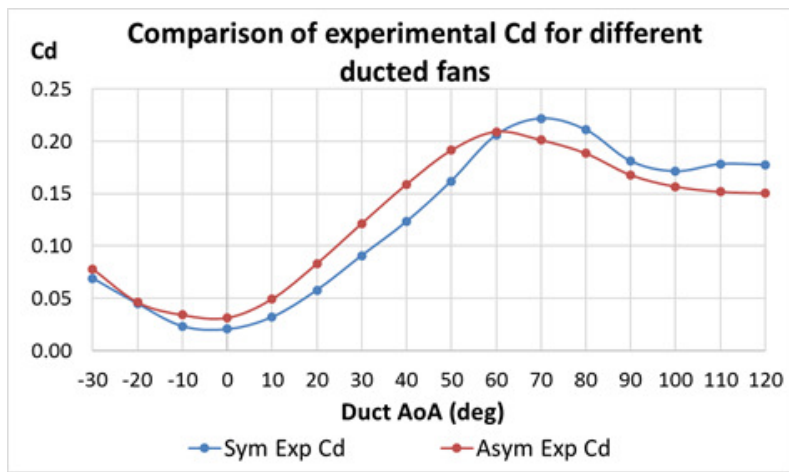

(b)

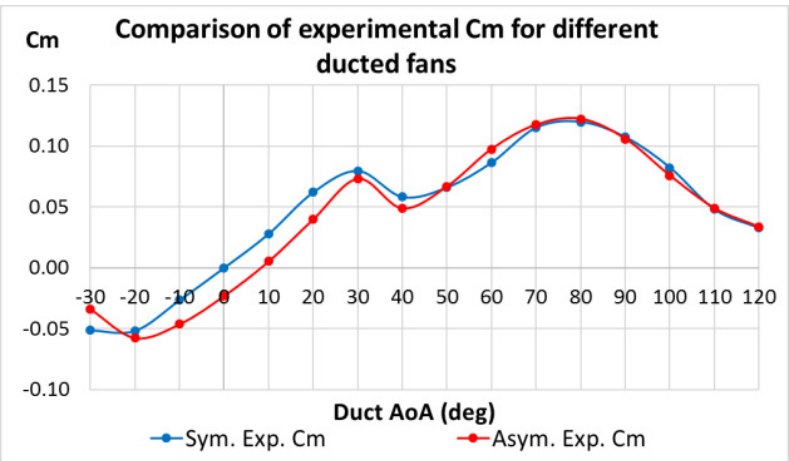

(c)

Figure 4-19: The variation of experimental aerodynamic coefficients for symmetrical and asymmetrical ducted fans under the influence of active fans in different AoA; (a) $C_{l}$, (b) $C_{d}$, and (c) $C_{m}, \operatorname{Re}=226,000$.

\subsection{Tilting ducted fans attached to the wing tips of proposed VTOL UAV in transition}

Simulation of resultant horizontal and vertical forces and pitching moments is the first step for investigation of the stability and control of the proposed VTOL UAV. The wing has the main role of lift generation. In addition, the elevators provide longitudinal stability by balancing pitching moments about the centre of gravity of the aircraft. The wing's lift and tail's pitching moment are functions of AoA and freestream velocity $\left(L_{W}=1 / 2 \rho S U^{2} C_{L_{W}}\right.$ and $\left.M_{H T}=1 / 2 \rho S U^{2} C_{L_{H T}} h_{H T}\right)$. As the wing's lift and horizontal tail's pitching moment are functions of the velocity $U^{2}$, they can operate efficiently only if the proposed VTOL UAV flies higher than a specific cruise velocity.

As shown in Figure 4-18, ducted fans' lift and drag increase as AoA increases during transition from cruise mode to hover. An increment in drag force results in decrement in the velocity of aircraft, decrement of lift generations by wing, and pitching moment by the horizontal tail. In order 
to have a level flight and for compensation of the loss in the wing's lift, it is necessary to increase thrust in higher AoAs. This could be done only by increasing the power delivered to the propeller. An increment in the power and induced velocity of incoming flow to the rotor plane will change all aerodynamic forces and pitching moments, thrust, ram drag and its related pitching moment. Thus, it is necessary to provide all forces and pitching moments by real time calculations. The most important parameter for real time calculation is the angular velocity $(\omega=\dot{\alpha})$ of tilting ducted fans attached to the wing tips. The aerodynamic response of the proposed VTOL UAV during transition condition is out of the scope of this research and it is another area that requires more investigation.

To have an initial pattern for changes in the resultant forces and pitching moments of the proposed VTOL UAV, changes in the aerodynamic coefficients of symmetrical and asymmetrical ducted fans are compared in Figure 4-20 for tilting rate of $\omega=10 \mathrm{deg} / \mathrm{sec}$. These coefficients are calculated for tilting ducted fans in the uniform flow field with $U_{x}=15 \mathrm{~m} / \mathrm{s}$ in the range of $-30^{\circ}$ to $120^{\circ}$ for AoA by running ANSYS-Fluent software in transient mode (see Appendix H). These parameters were calculated previously in stationary conditions and their comparisons are shown in Figure 4-14.

As can be seen, the fluctuations in aerodynamic coefficients during transition from cruise mode to hover happens when AoA is larger than $40^{\circ}$ (Time $>9 \mathrm{sec}$ ) for symmetrical ducted fan. This happens for asymmetrical ducted fan when AoA is larger than $50^{\circ}$ (Time $>10$ sec). These fluctuations cannot be captured in stationary conditions by CFD simulation, which were shown in all previous sections. Comparing transitional and stationary CFD simulations shows trends for changes in aerodynamic coefficients are similar and they are most likely in similar values. 


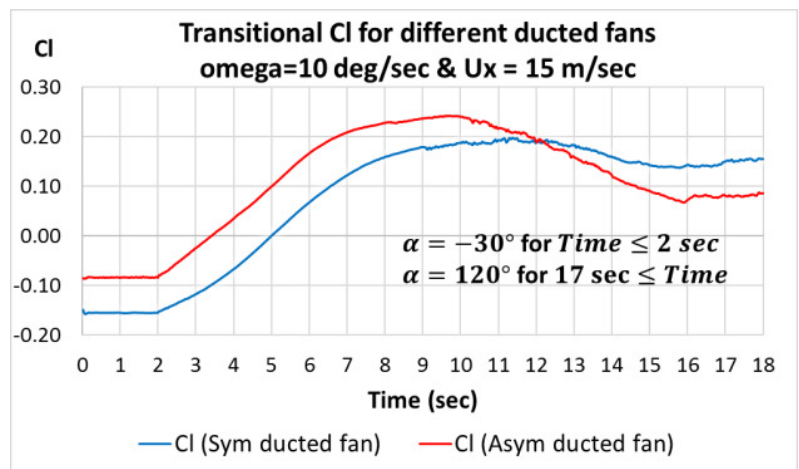

(a)

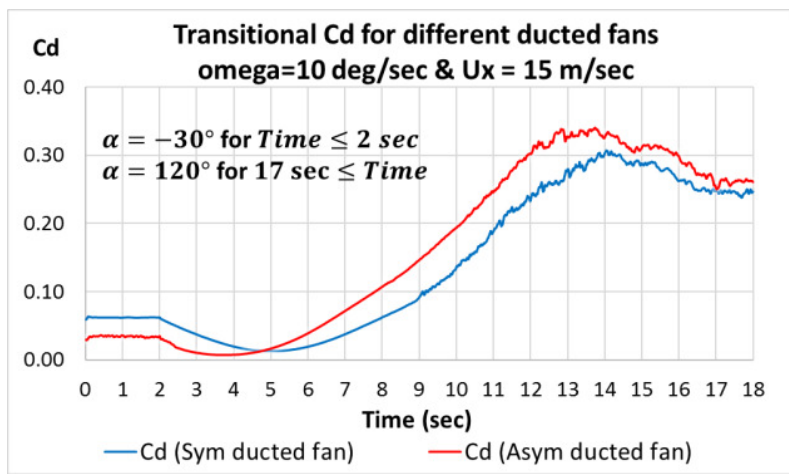

(b)

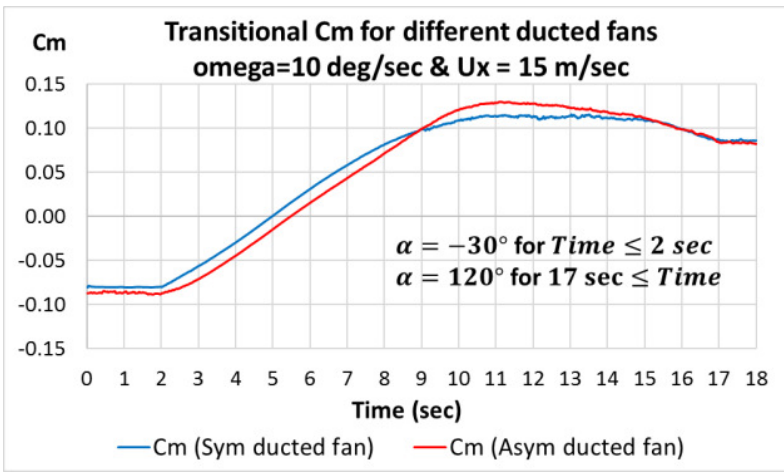

(c)

Figure 4-20: Comparing aerodynamic coefficients for symmetrical and asymmetrical ducted fans in transition from $\alpha=-30^{\circ}$ to $\alpha=120^{\circ}$ with $\omega=10 \mathrm{deg} / \mathrm{sec}$ and $U_{x}=15 \mathrm{~m} / \mathrm{s}$; (a) $C_{l}$, (b)

$$
C_{d} \text { and (c) } C_{m}
$$

The resultant transitional forces and pitching moment on the ducted fans are compared in Figure

4-21. Based on the changes in the resultant aerodynamic forces and pitching moment of ducted fans, it is possible to calculate all forces and pitching moment for the proposed VTOL UAV in transition condition. Calculating these forces and pitching moment for the proposed VTOL UAV can be done by knowing the necessary aerodynamic coefficients and parameters of the proposed VTOL UAV. 


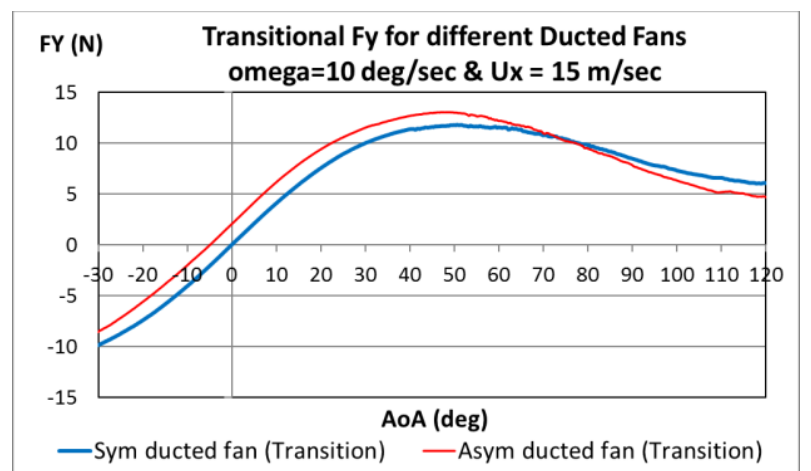

(a)

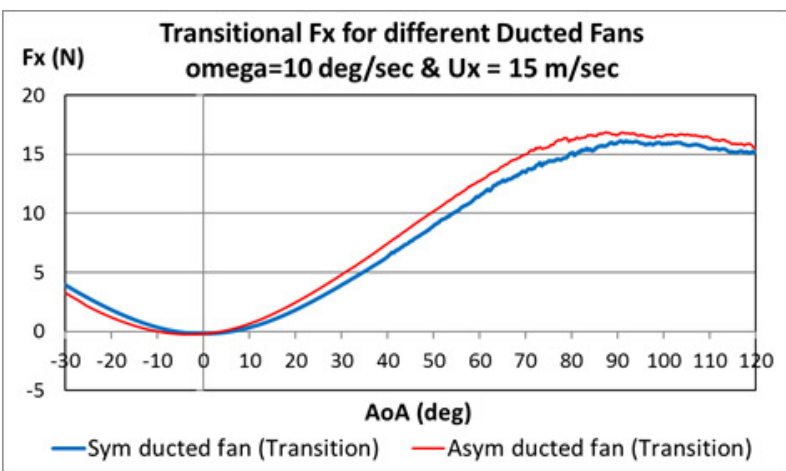

(b)

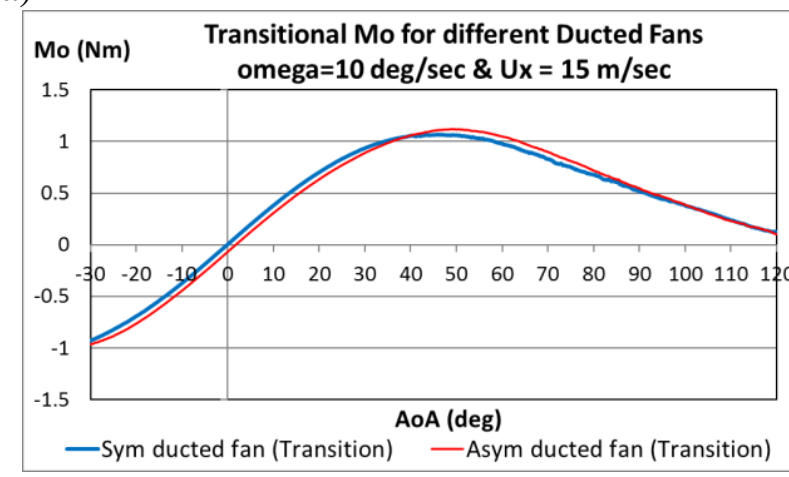

(c)

Figure 4-21: Comparing resultant forces and moments on symmetrical and asymmetrical ducted fans in transition from $\alpha=-30^{\circ}$ to $\alpha=120^{\circ}$ with $\omega=10 \mathrm{deg} / \mathrm{sec}$ and $U_{x}=15 \mathrm{~m} / \mathrm{s}$; (a) $F_{y}$, (b) $F_{x}$ and (c) $M_{o}$

\subsection{The effects of changes in the tilting rate of ducted fans in transition mode}

Three different rates of tilting $(\omega=10,20 \& 30 \mathrm{deg} / \mathrm{sec})$ were assumed and the aerodynamic coefficients for the tilting symmetrical and asymmetrical ducted fans were calculated by using CFD modeling. The variations of tilting angle for all simulations were in the range of $-30^{\circ} \leq \alpha \leq$ $120^{\circ}$. The induced velocity $\left(V_{\text {ind }}\right)$ for each ducted fan in different AoAs was computed by "User Defined Functions" in ANSYS-Fluent software (see Appendix H). Comparison of the results shows changes in the rate of tilting do not have significant effects on the aerodynamic coefficients in the range of $-30^{\circ} \leq \alpha \leq 40^{\circ}$ and their values are the same as stationary condition in that range (see aerodynamic coefficients of symmetrical and asymmetrical ducted fans in Figure 4-14). Fluctuations in the aerodynamic coefficients can be seen in the range of $40^{\circ} \leq \alpha$ for the 
symmetrical ducted fan and in the range of $50^{\circ} \leq \alpha$ for the asymmetrical ducted fan as shown in Figure 4-22.

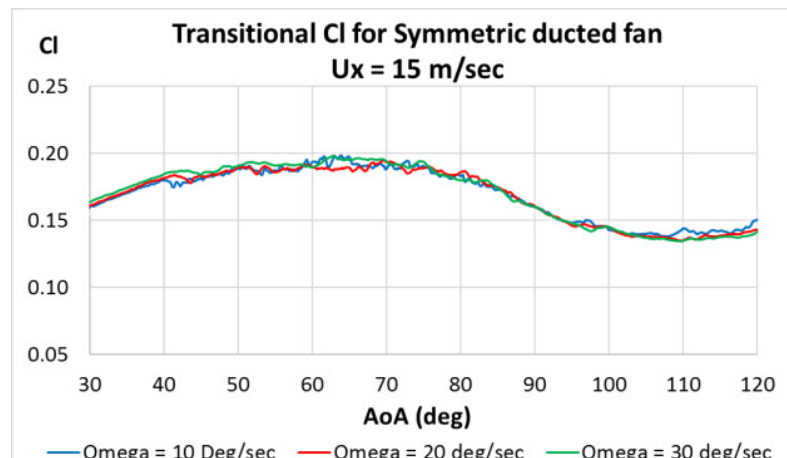

(a)

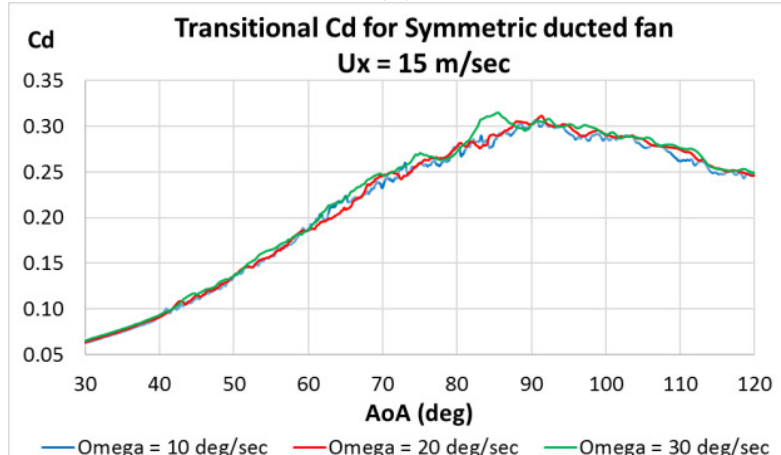

(b)

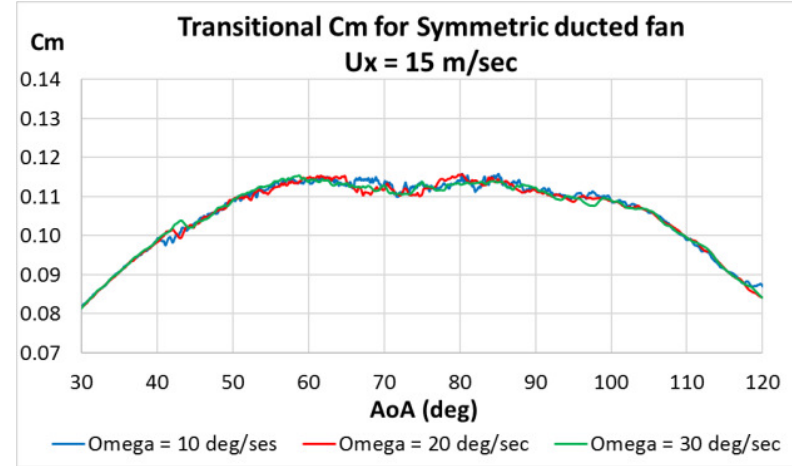

(c)

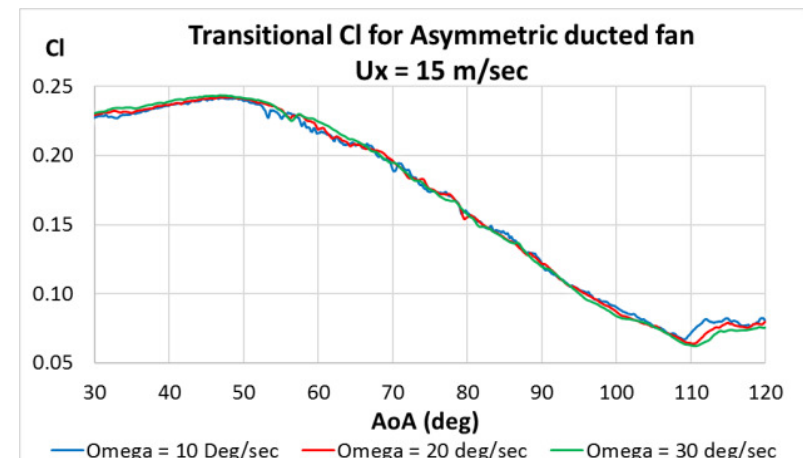

(d)

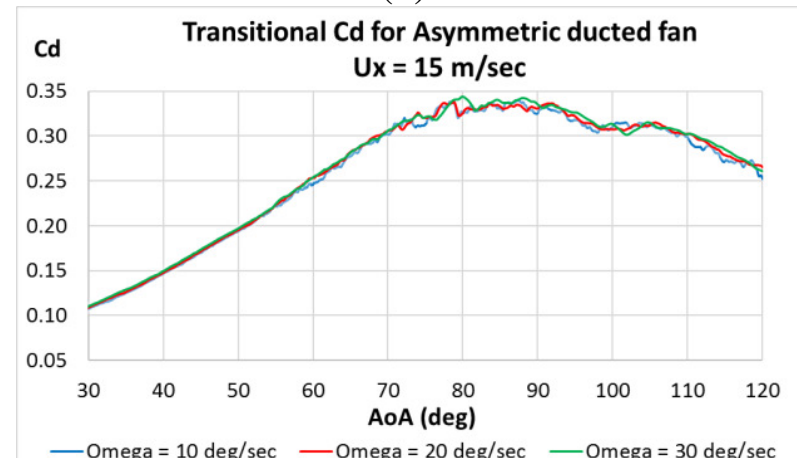

(e)

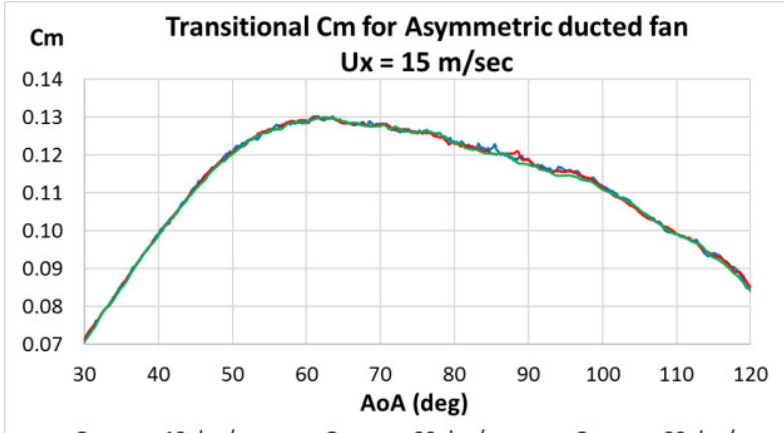

(f)

Figure 4-22: The effects of changes in the tilting rate on the transitional aerodynamic coefficients for symmetrical and asymmetrical ducted fans: (a \& d) $C_{l}$, (b \& e) $C_{d}$ and (c \& f) $C_{m}$ in three different tilting rates: $\omega=10,20 \& 30 \mathrm{deg} / \mathrm{sec}, 30^{\circ} \leq \alpha \leq 120^{\circ}$, and $U_{x}=15 \mathrm{~m} / \mathrm{s}$ 
The separation of flow around the windward duct lip has strong effects on distortion of the velocity magnitude at the duct's inlet [11]. The occurrence of cyclic fluctuations in aerodynamic forces can be expressed by following three main reasons:

1- The non-uniform flow separation in higher AoAs starts from the leading edge of the duct lip and generates strong vorticities.

2- These strong vorticities distort the streamlines of the flow toward the rotor plane and generate strong turbulent fluctuations around the duct lip and in the plane of the rotor.

3- The turbulent fluctuation affects pressure distribution at the rotor plane and generates cyclic disturbance in the aerodynamic forces of the ducted fan.

As shown in Figure 4-23, the cyclic disturbance in the aerodynamic coefficients affects the resultant forces and pitching moment of the ducted fans and creates sudden shakes by increasing AoA during transition period. These sudden shakes were observed even in the stationary conditions for higher AoAs in the range of $90^{\circ} \leq \alpha \leq 120^{\circ}$ in the wind tunnel experiments.

As it was shown in Figure 4-22, changes in the tilting rate affected both lift and drag coefficients, and it was expected to have disturbances in both vertical and horizontal components of the resultant forces, as shown in Figure 4-23.

According to Figure 4-22, increment in the tilting rate increases the amplitude of the fluctuations in the aerodynamic coefficients for both ducted fans. The amplitudes of these fluctuations are relatively larger for symmetrical ducted fan as its curvature on the upper side is less than that on the asymmetrical ducted fan. Thus, the separated flow has stronger effects on the symmetrical ducted fan. 


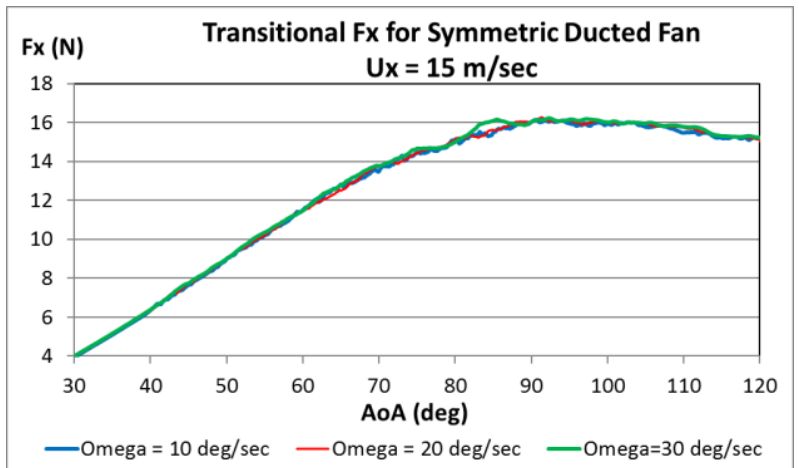

(a)

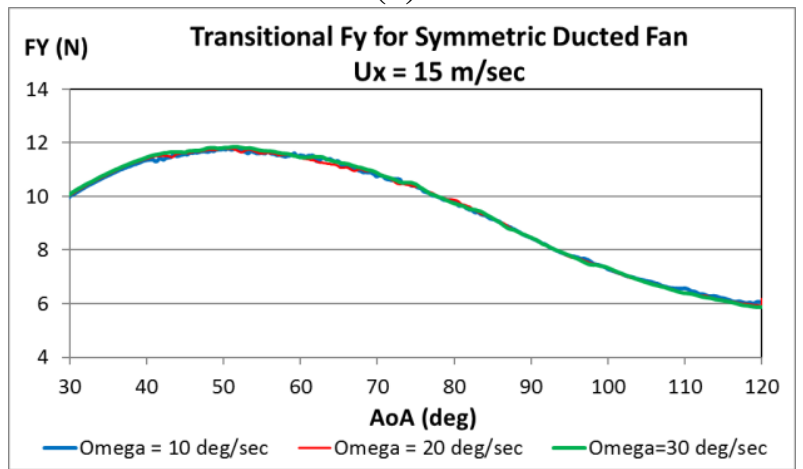

(b)

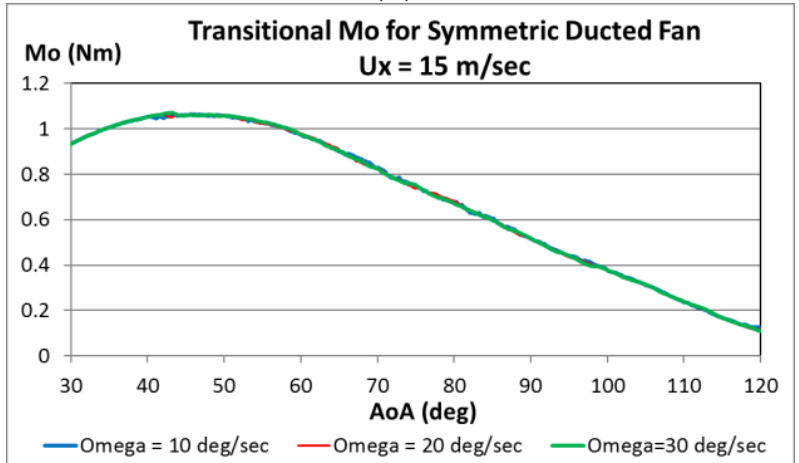

(c)

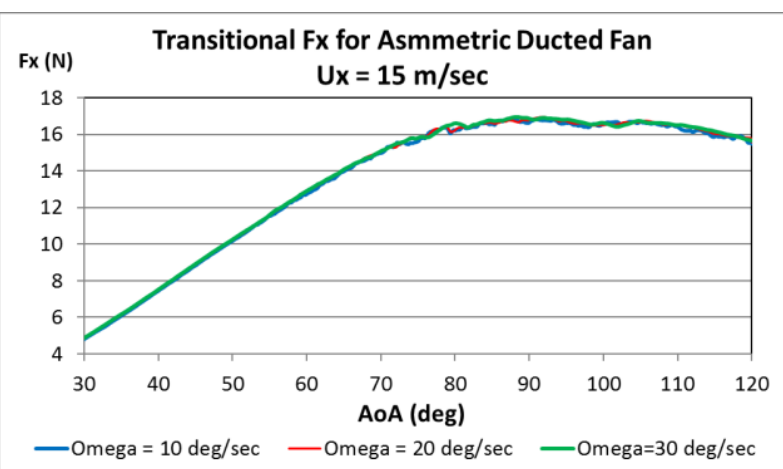

(d)

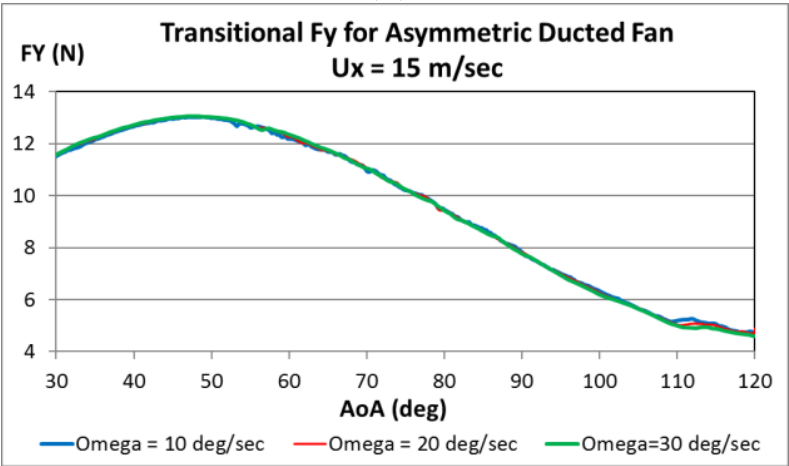

(e)

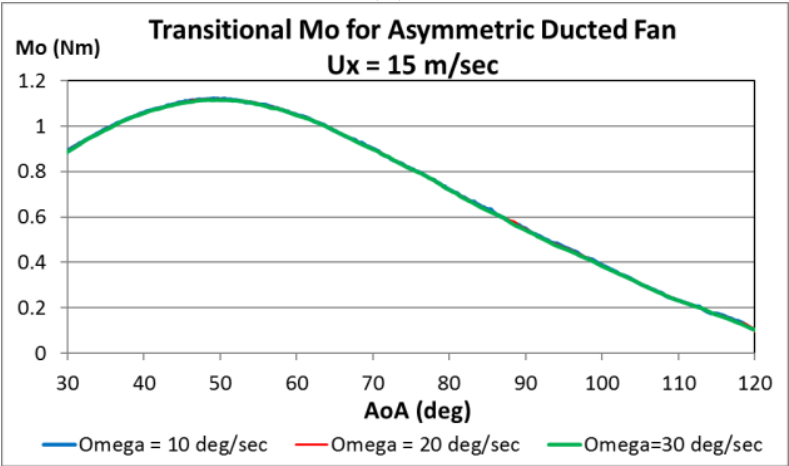

(f)

Figure 4-23: The effects of tilting rate changes on the transitional resultant forces and pitching moment of the symmetrical and asymmetrical ducted fans: (a \& d) $C_{l}$, (b \& e) $C_{d}$ and (c \& f) $C_{m}$ in three different rates: $\omega=10,20 \& 30 \mathrm{deg} / \mathrm{sec}, 30^{\circ} \leq \alpha \leq 120^{\circ}$, and $U_{x}=15 \mathrm{~m} / \mathrm{s}$

\subsection{Proposed VTOL UAV in the transition from cruise mode to hover}

The specifications of the proposed VTOL UAV with two ducted fans installed on its wing tips are listed in Table I. 1. The details for calculation of the specifications are presented in the Appendix I. Based on the selected tilting rate $(\omega)$ and type of ducted fan, the provided MATLAB 
codes (see Appendix J) calculate the proposed VTOL UAV's velocity by using trial and error method to satisfy equation (3.45). The final travelled time for completing transition from cruise mode to hover for proposed VTOL UAV by using two different ducted fans and for three different tilting rates are presented in the Table 4.3.

Table 4.3: The final travelled time for transition from cruise mode to hover for proposed VTOL

\begin{tabular}{|c|c|c|c|}
\hline \multicolumn{5}{|c|}{ UAV } \\
\hline Wing tips ducted fans & $\omega=10 \mathrm{deg} / \mathrm{sec}$ & $\omega=20 \mathrm{deg} / \mathrm{sec}$ & $\omega=30 \mathrm{deg} / \mathrm{sec}$ \\
\hline Symmetrical & $11.15 \mathrm{sec}$ & $6.29 \mathrm{sec}$ & $4.73 \mathrm{sec}$ \\
\hline Asymmetrical & $11.02 \mathrm{sec}$ & $6.27 \mathrm{sec}$ & $4.68 \mathrm{sec}$ \\
\hline
\end{tabular}

After calculating the UAV's velocity and based on the spending time during transition, the other parameters can be found as following:

Tilting angle of ducted fans $(\boldsymbol{\alpha})$ : Figure 4-24 shows the variation of tilting angles of the ducted fans during transition for different tilting rates. There is no major difference between tilting angles of the symmetrical and asymmetrical ducted fans for each specific tilting rate. The tilting angle should pass $90^{\circ}$ to create essential braking force for stopping the VTOL UAV.

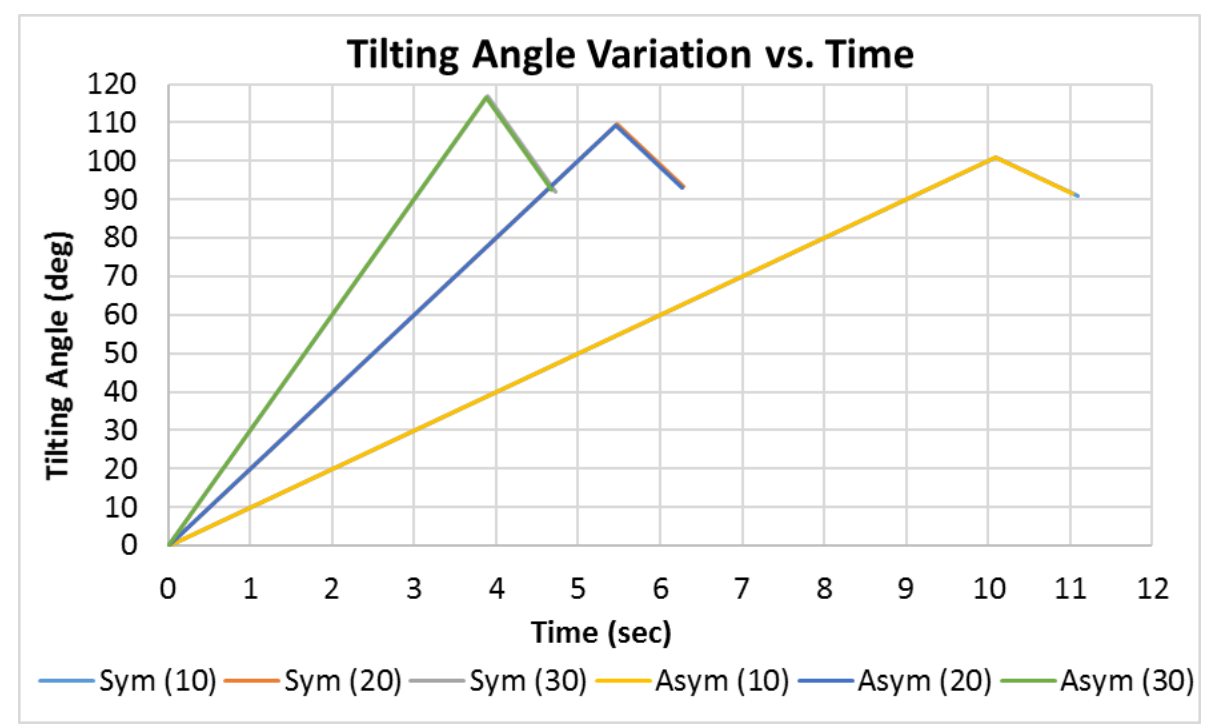

Figure 4-24: The variation of tilting angle of the ducted fans during transition for symmetrical and asymmetrical ducted fans in three different tilting rates $(\omega=10,20 \& 30 \mathrm{deg} / \mathrm{sec})$ 
By increasing tilting rate, the transition can be done in shorter time and this needs larger braking force, which can be produced in the higher tilting angles. The maximum tilting angle for each ducted fan in different tilting rates are listed in the Table 4.4. As shown in the Figure 4-24, ducted fans have to come back to $\alpha=90^{\circ}$ in the last step of transition to get into stable hover condition.

Table 4.4: Maximum tilting angle of ducted fans in the transition from cruise mode to hover

\begin{tabular}{|c|c|c|c|}
\hline Type of ducted fan & $\omega=10 \mathrm{deg} / \mathrm{sec}$ & $\omega=20 \mathrm{deg} / \mathrm{sec}$ & $\omega=30 \mathrm{deg} / \mathrm{sec}$ \\
\hline Symmetrical & $\alpha_{\max }=100.9^{\circ}$ & $\alpha_{\max }=109.6^{\circ}$ & $\alpha_{\max }=117^{\circ}$ \\
\hline Asymmetrical & $\alpha_{\max }=100.8^{\circ}$ & $\alpha_{\max }=109.2^{\circ}$ & $\alpha_{\max }=116.4^{\circ}$ \\
\hline
\end{tabular}

Distance travelled during transition: As illustrated in Figure 4-25, the travelled distance during transition is shorter (max. $2.5 \%$ ) by applying higher tilting rates. The travelled distance is relatively shorter by using asymmetrical ducted fan for proposed VTOL UAV.

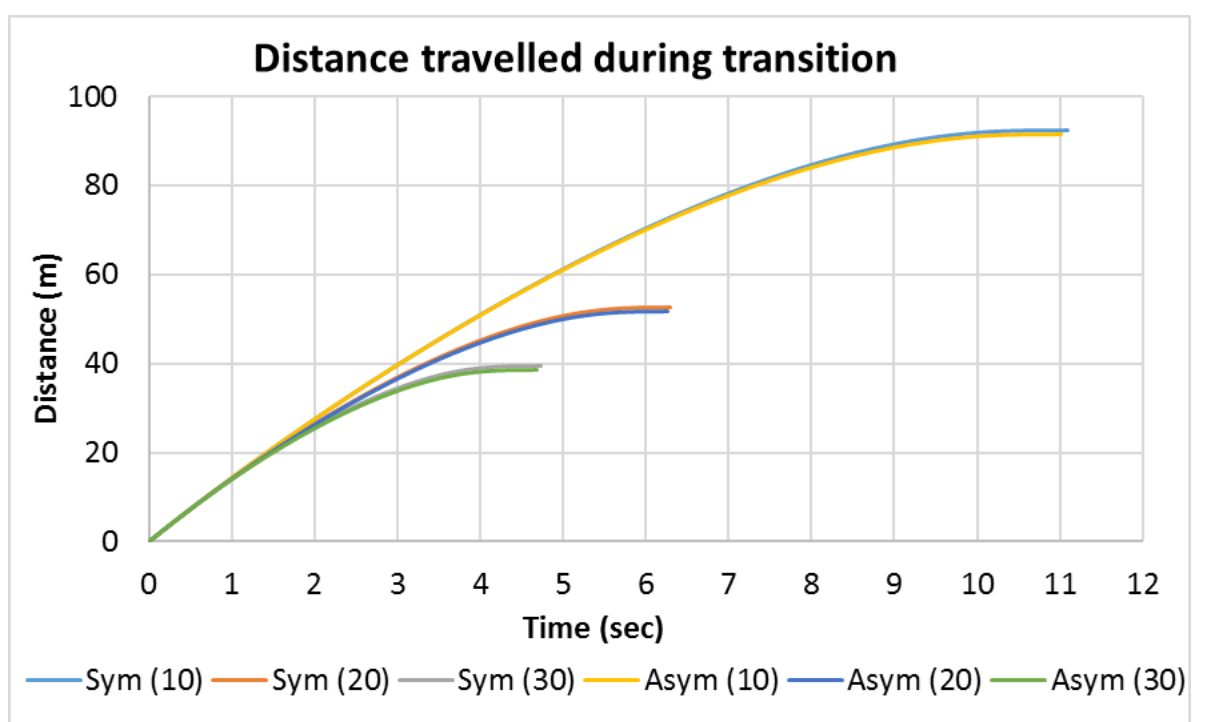

Figure 4-25: The variation of VTOL UAV's travelled distance during transition by using symmetrical and asymmetrical ducted fans in different tilting rates $(\omega=10,20 \& 30 \mathrm{deg} / \mathrm{sec})$ 
The velocity of the VTOL $\boldsymbol{U A V}(\boldsymbol{U})$ : As shown in the Figure 4-26, the VTOL UAV has shorter transition time by increasing tilting rate. The initial velocity of the VTOL UAV is $U_{t=0}=15 \mathrm{~m} / \mathrm{s}$ just before starting transition and it is zero at hover $\left(U_{\text {hover }}=0\right)$.

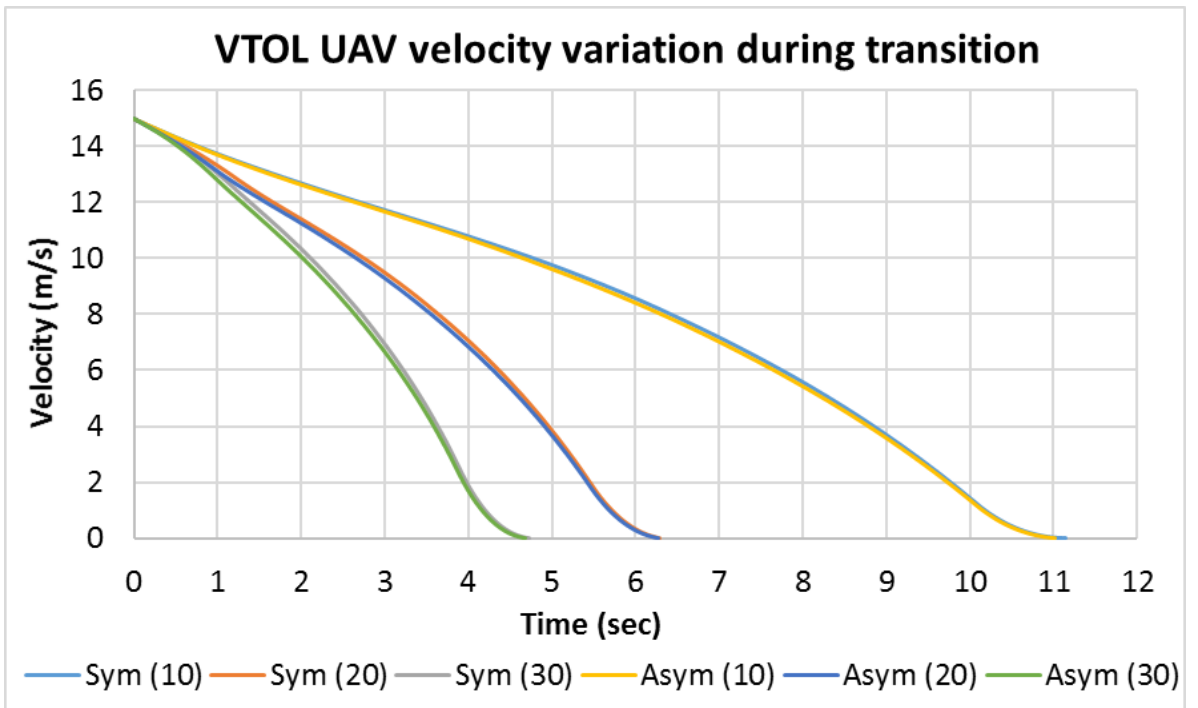

Figure 4-26: The variation of VTOL UAV's velocity during transition by using symmetrical and asymmetrical ducted fans in three different tilting rates $(\omega=10,20 \& 30 \mathrm{deg} / \mathrm{sec})$

The $x$-acceleration of the VTOL UAV: As illustrated in the Figure 4-27, the negative values show deceleration of the VTOL UAV during transition. The higher tilting rates means faster transition and stronger braking force to stop VTOL UAV in shorter distance. Comparison shows the type of ducted fan does not have major effects on the deceleration. The maximum deceleration occurs when the x-component of thrust $(T \cos \alpha)$ generates the maximum braking force in the range of $\alpha>90^{\circ}$. 


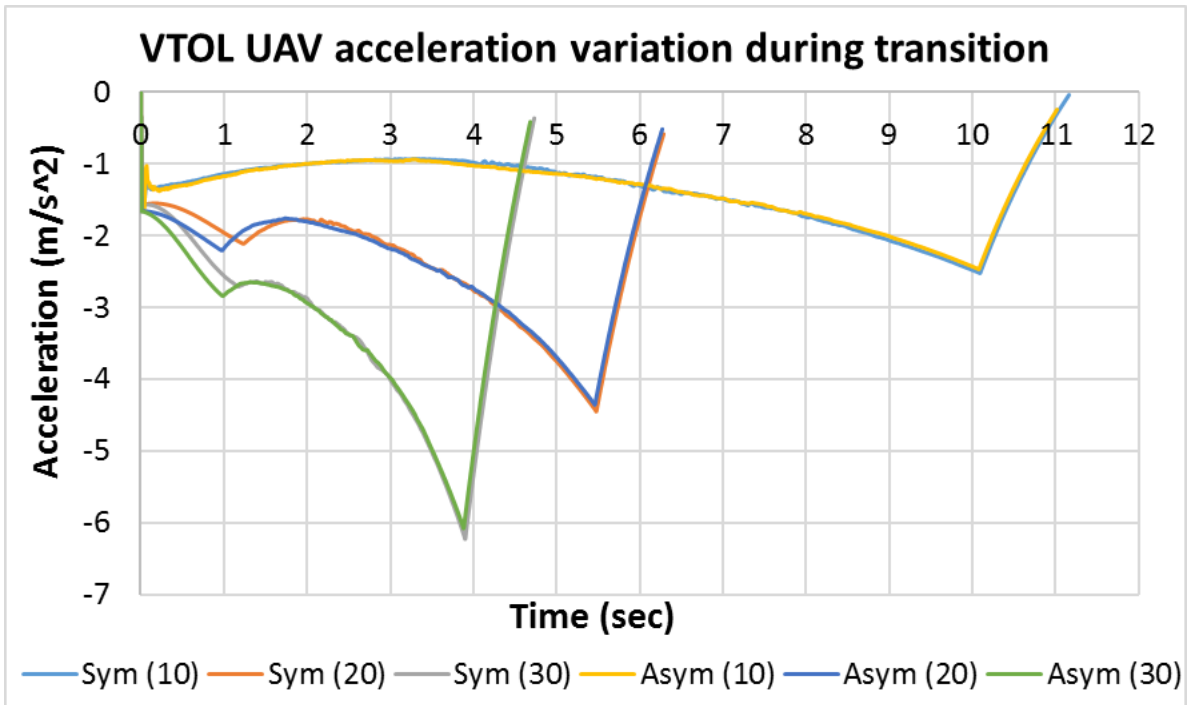

Figure 4-27: The variation of VTOL UAV's x-acceleration during transition by using symmetrical and asymmetrical ducted fans in different tilting rates $(\omega=10,20 \& 30 \mathrm{deg} / \mathrm{sec})$

The trust variation: As presented in the Figure 4-28, after a sudden reduction in the beginning of the transition, the ducted fan's thrust starts increasing based on the time and tilting rate of the ducted fan. Thrust works like a braking force during transition to reduce the VTOL UAV's velocity. Although each engine should produce $18.058 \mathrm{~N}$ force in hover, they need to produce higher thrust (up to 15\%) to stop the VTOL UAV during transition in a shorter distance.

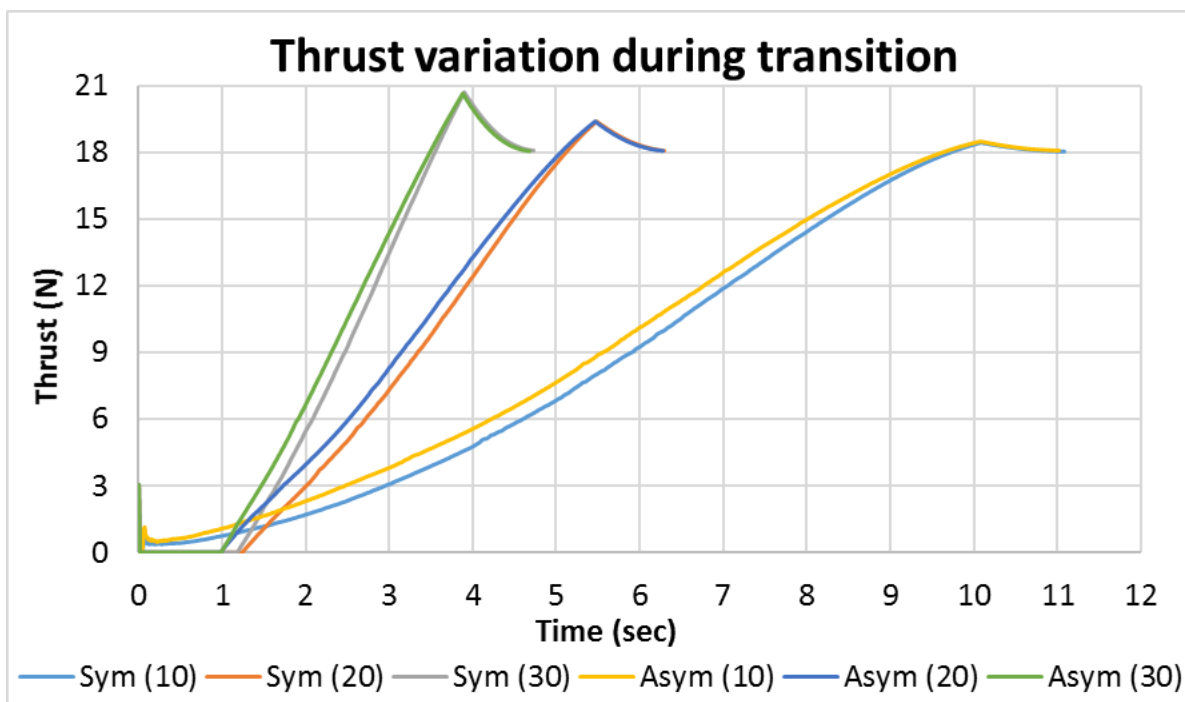

Figure 4-28: The variation of ducted fans thrust (for one engine) during transition by using symmetrical and asymmetrical ducted fans in different tilting rates $(\omega=10,20 \& 30 \mathrm{deg} / \mathrm{sec})$ 
Changes in the pitching moment: Figure 4-29 shows additional generated pitching moment during transition from cruise mode to hover of the proposed VTOL UAV in this research. This transitional pitching moment is generated due to the changes in the aerodynamic forces and pitching moments of the wing, tilting ducted fans, and other components. The asymmetrical ducted fan with larger tilting rates generates higher transitional pitching moments compare to the symmetrical ducted fan. The additional generated pitching moment should be cancelled by tail's elevator during transition, as it can increase the AoA of the VTOL UAV in a short time and change level flight to a sudden ascending flight, which can be ended with a stall condition.

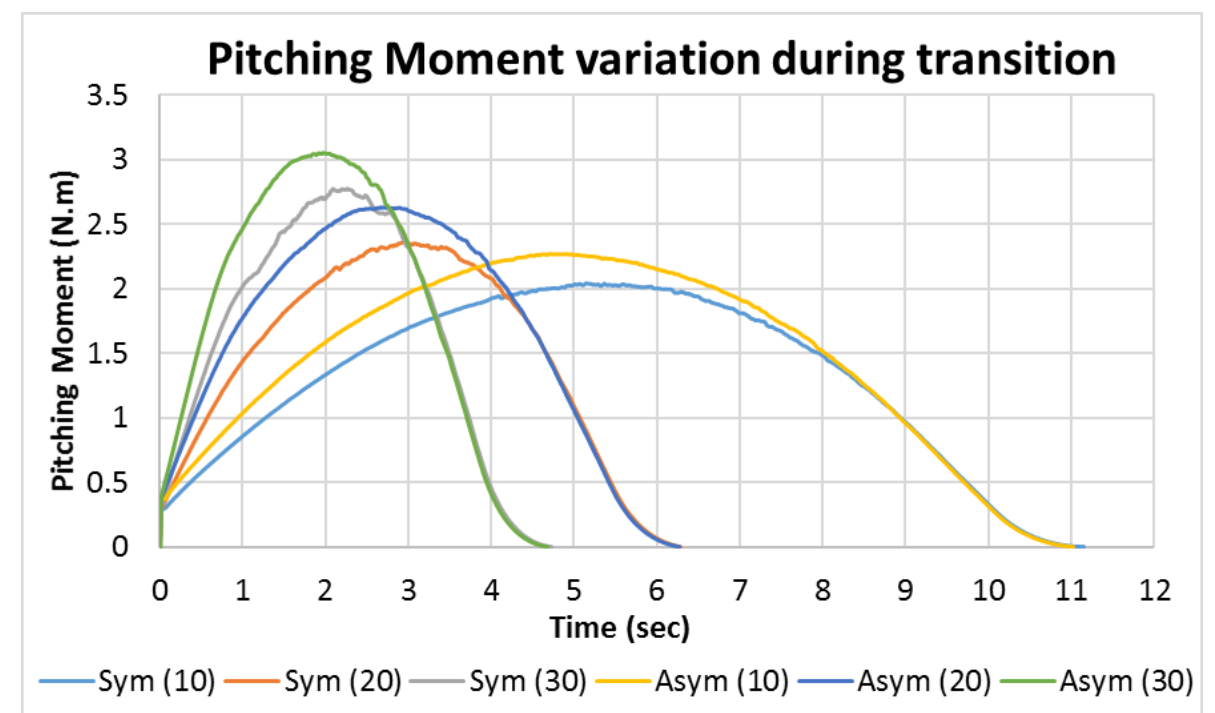

Figure 4-29: The changes in the pitching moments during transition by using symmetrical and asymmetrical ducted fans in three different tilting rates $(\omega=10,20 \& 30 \mathrm{deg} / \mathrm{sec})$

As it illustrated in the Figure 4-29, the proposed VTOL UAV with symmetrical ducted fan has been affected by larger fluctuations in the transitional pitching moments and in the higher tilting rates. These fluctuations in the transitional pitching moments are results of flow separation and turbulence fluctuation when tilting angle of the symmetrical ducted fan is in the range of $40^{\circ}<$ $\alpha<85^{\circ}$ as discussed before in the section 3.9. 


\section{CONCLUDING REMARKS}

\subsection{Conclusion}

In this research, the aerodynamics of two ducts (one symmetrical and one asymmetrical) were studied by CFD and experimental methods in different conditions. The aerodynamic forces and pitching moment acting on these two ducts were compared to find out the advantages and disadvantages of using them as tilting ducted fan installed on the wing tips of the proposed VTOL UAV in this research. Using this configuration of the ducted fans can minimize the angle between the thrust line and flight path of the VTOL UAV. This enhancement provides uniform flow to the rotor disk, higher thrust, less drag, and faster forward velocity, which results in longer range and endurance in cruise mode.

Four objectives were defined in this research as follows:

1- Investigation for the use of asymmetrical shape for the external body of ducted fans,

2- Prediction of the induced velocity of the inlet flow to the rotor plane, to conduct CFD modeling for ducted fans,

3- Using CFD modeling for predicting aerodynamic forces and pitching moments of the tilting ducted fans in the transition conditions for different tilting rates,

4- Simulation of the level flight conditions of the proposed VTOL UAV during transition between cruise mode and hover.

It was necessary to define several procedures to obtain CFD simulations and experimental tests in this research. Different meshes were designed using GAMBIT software to perform CFD simulations for the two tilting ducts with/without fans in different conditions. The all CFD simulations were performed in steady flow with $U=15 \mathrm{~m} / \mathrm{s}$ and $\mathrm{Re}=226,000$ and tilting angle in the range of $-30^{\circ} \leq A o A \leq 120^{\circ}$. 
To conduct the experiments, the actual models of two ducts were designed and printed using CATIA software and a 3D printer. A supporting frame was designed for attaching ducts to it, providing tilting motion for ducts through a gear system and measuring AoA by a $360^{\circ}$ protractor. By connecting the supporting frame to the load measuring system of the wind tunnel, lift, drag, and pitching moments of two ducts with/without fans could be recorded in stationary conditions for the same range of AoAs and free stream velocity were used in the CFD modeling.

For the first objective of this research, the ANSYS-Fluent software simulated the aerodynamic forces and pitching moment acting on the ducts without fan in stationary conditions for a wide range of AoAs by using standard $k-\varepsilon$ turbulence modeling.

Then, the ducts without fans were tested in the wind tunnel. The lift, drag, and pitching moment from experiments were compared with the CFD results showing good agreements between the two methods. The results from both methods show that the external shape of the asymmetrical duct has the main role of generating higher lift in cruise mode and a wide range of AoAs $\left(-30^{\circ} \leq A o A \leq\right.$ $40^{\circ}$ ). The drag forces are very similar for both ducts in cruise mode and wide range of AoAs. Due to the smaller curvature of the lower surface of the asymmetrical duct, its pitching moment is slightly higher than that for the symmetrical duct during the transition in the range of $20^{\circ} \leq A o A \leq$ $80^{\circ}$

The second objective needed to calculate the induced velocity to the rotor plane of the ducted fans by assuming constant power delivered to the propeller and using actuator disk model. Then, by using the calculated values for induced velocity in different AoAs, the aerodynamic forces and pitching moments of ducted fans were obtained in stationary conditions using ANSYS-Fluent software. These CFD simulations were done in steady flow for the same conditions of all the tests and using RNG (Re-Normalisation Group) $k-\varepsilon$ turbulence modeling. The fan's thrust, ram drag 
and its related pitching moment were calculated using direct equations and added to aerodynamic forces and pitching moments of ducted fans to obtain final resultant horizontal and vertical forces and pitching moments in the CFD simulations.

To evaluate the CFD results, the ducts were equipped with electromotor and propellers, and tested in different AoAs in the wind tunnel. The experimental horizontal and vertical forces and pitching moment of the ducted fans were compared with the resultant forces and pitching moment from the CFD modeling, and satisfactory agreements between the two methods were observed.

The third objective was achieved using the ANSYS-Fluent software in transient mode. The resultant horizontal and vertical forces and pitching moments of two tilting ducted fans were obtained in three different tilting rates $(\omega=10,20 \& 30 \mathrm{deg} / \mathrm{sec})$. The unexpected cyclic turbulent fluctuations in the aerodynamic forces and pitching moments were observed for both ducted fans in the higher AoAs. Increment in the tilting rate increased the amplitude of the fluctuations, which relatively are larger for symmetrical ducted fan.

Finally, the fourth objective was achieved by designing the proposed VTOL UAV with two ducted fans installed on its wing tips. The resultant aerodynamic forces and pitching moments acting on the proposed VTOL UAV were calculated in three different tilting rates and using two different types of ducted fans. The final results showed asymmetrical ducted fans can provide smoother transition flight between cruise mode and hover.

The final findings of this research can be listed as following:

1. Due to the specific shape, the asymmetrical ducted fan can generate additional lift in cruise mode, which can help to reduce wing area and the consequent weight and drag. This enhancement will help to increase the range and endurance of the flight for the VTOL UAV. 
2. The method for predicting induced velocity to the rotor plane $\left(V_{\text {ind }}\right)$ is valid and can be used to provide real time calculations of parameters based on the angular velocity $(\omega)$ of the tilting ducted fans.

3. The increment in the tilting rate increases the amplitude of the unexpected cyclic turbulent fluctuations in the aerodynamic coefficients of the tilting ducted fans in higher AoAs.

4. Due to smaller fluctuations in the aerodynamic coefficients, the asymmetrical ducted fan can provide smoother transition flight between cruise mode and hover.

\subsection{Contributions}

The main area of the contribution of this research is in the aerodynamics of asymmetrical ducted fans. Many activities were done to show the advantage of using asymmetrical ducted fan in this research. As it was mentioned before, to the best of my knowledge, there was no specific published study for using an asymmetrical shape for external body of the ducted fans.

The contribution of this research can be summarized in the following categories:

1. Application of asymmetrical ducted fan: Results from the experiments and CFD modeling showed asymmetrical shape can be used for the external body of ducted fans to generate additional lift in cruise mode. This can help to reduce wing area and the consequent weight and drag. More than that, the aerodynamically performance of asymmetrical ducted fan in transition from cruise mode to hover is better than symmetrical ducted fan.

2. Methodology development: This involved the combination of "actuator disk model" combined with the assumption of "constant power delivered to the propeller" to calculate the induced velocity to the rotor plane of the ducted fans. This is essential for studying the aerodynamic coefficients of ducted fans in different AoAs when a large amount of the air 
is forced to pass through the ducted fan. This model was used to obtain the aerodynamic coefficients of tilting ducted fans in different tilting rates and study the effects of the tilting rate changes.

3. Prediction, analysis and validation: Both symmetrical and asymmetrical ducted fans were studied by CFD simulations and experiments. The experimental results validated both the CFD modeling and the method for predicting induced velocity to the rotor plane of the ducted fans.

\subsection{Future work}

Future works can be research on the following topics:

1- Optimization of the ducted fans (inlet and outlet diameters, length of the ducts, profile of the airfoils for symmetrical and asymmetrical ducts, ...)

2- Experiments on the unsteady aerodynamics of the actual model of tilting ducted fans and effects of changes in their tilting rates

3- Experiments on the stability and control of an actual model of the proposed VTOL UAV during different maneuvers such as yaw in hover and roll and steady banked turn in cruise mode and also transition between cruise mode and hover. 


\section{Appendices}

Appendix A: More about the turbulence modeling

Appendix B: Geometry of meshes

Appendix C: Designing, producing, and preparing actual models of ducted fans

Appendix D: Wind tunnel facility

Appendix E: Measuring aerodynamic forces on the frame and ducts with/without fans

Appendix F: Measuring pitching moments of the ducts' weights

Appendix G: Error analysis for measuring aerodynamic coefficients

Appendix H: Summary of ANSYS-Fluent settings in transient mode and User Defined Functions

Appendix I: The specifications of the proposed VTOL UAV with two ducted fans

Appendix J: MATLAB code for calculating flight parameters of proposed VTOL UAV during transition between cruise mode to hover 


\section{Appendix A: More about the turbulence modeling}

\section{Comparison between different turbulence modeling}

It was mentioned in the section 3.7 that the $k-\varepsilon$ turbulence modeling was used in the simulations for ducts without fans and the RNG $k-\varepsilon$ turbulence modeling was used in the simulations for ducted fans. Figure A. 1 shows the comparison between experimental lift and drag of the symmetrical duct without fan with three turbulence modeling used by ANSYS-Fluent. The results from Spalart-Allmaras and $k-\omega$ turbulence modeling are far from the experimental results but the $k-\varepsilon$ turbulence modeling gives closer results to the experiments. Although the $k-\varepsilon$ turbulence modeling predicts the maximum lift at $A o A=30^{\circ}$, it gives very accurate value for lift in other AoAs. Also the drag force by $k-\varepsilon$ turbulence modeling shows closer values to the experimental drag force.

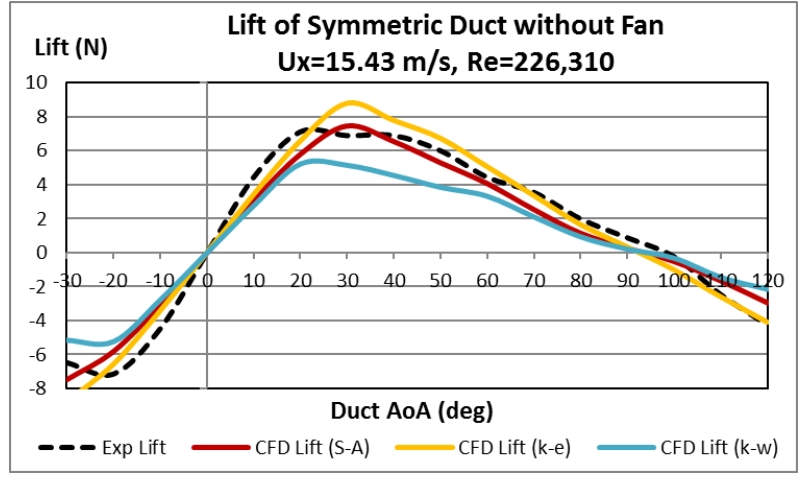

(a)

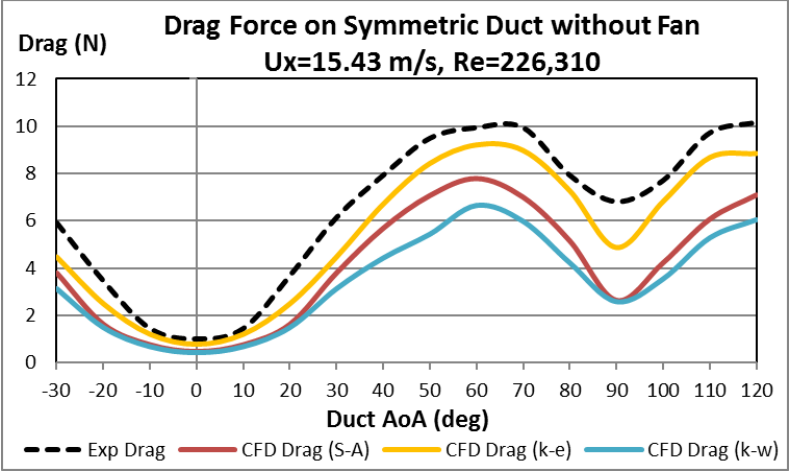

(b)

Figure A. 1: Comparison between different turbulence modeling and experimental results of symmetrical duct without fan; (a) lift, (b) drag

As the Spalart-Allmaras model is a one-equation model that solves a modeled transport equation for the kinematic eddy (turbulent) viscosity, it gives good results for boundary layers subjected to adverse pressure gradients [28]. Then, it is effectively a low-Reynolds number model and this could be the reason for computing drag lower than the experimental value in this research. 
The standard $k-\omega$ model is an empirical model transport equations for the turbulence kinetic energy $(k)$ and the specific dissipation rate $(\omega)$ which incorporates modifications for low-Reynolds number effects, compressibility, and shear flow spreading [29]. Although this model could predict the maximum lift at $A o A=20^{\circ}$, the lower calculated values for lift and drag by this model was the strong reason for not using it for turbulence modeling in this research.

The calculated forces by standard $k-\varepsilon$ and the RNG $k-\varepsilon$ turbulence modeling were compared with the experimental vertical and horizontal forces of the symmetrical ducted fan from the wind tunnel tests. The CFD simulations gave lift and drag of the symmetrical ducted fan and then the $\mathrm{y}$ - and $\mathrm{x}$-components of thrust and ram drag forces were calculated and added to the lift and drag to obtain final resultant forces in the vertical and horizontal directions. As seen in Figure A. 2, the RNG $k-\varepsilon$ turbulence modeling provides very accurate result for $F_{y}$. Also the resultant $F_{x}$ by RNG $k-\varepsilon$ turbulence modeling is similar to the experimental result for wide range of AoAs and it gives more accurate result than the standard $k-\varepsilon$ turbulence modeling in the higher AoAs.

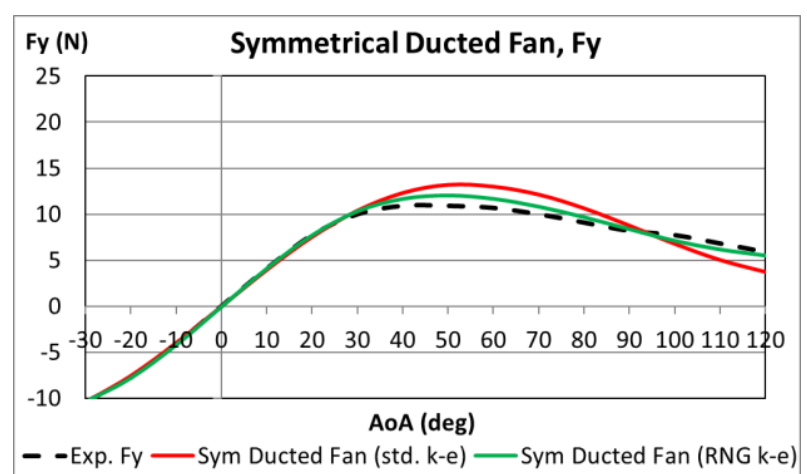

(a)

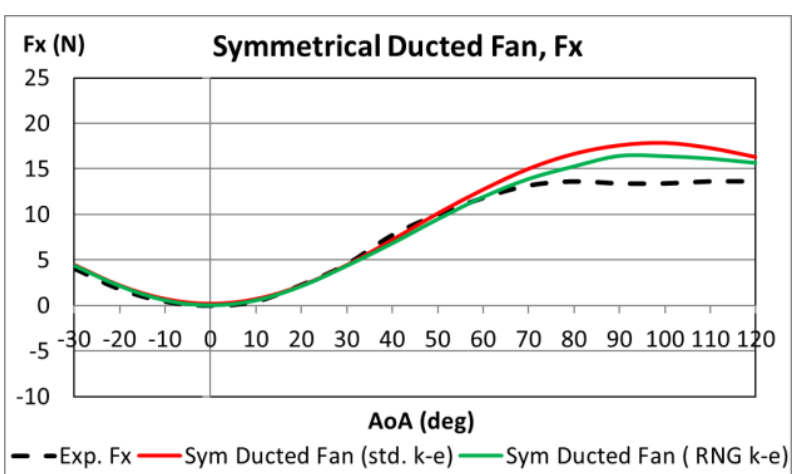

(b)

Figure A. 2: Comparison between standard and RNG $k-\varepsilon$ turbulence modeling with experimental results of symmetrical ducted fan; (a) Vertical forces, $F_{y}$, (b) Horizontal forces, $F_{x}$

\section{Eligibility of using $\boldsymbol{k}-\varepsilon$ turbulence modeling in the separated flow}

The flow separation is the result of adverse pressure gradient [30]. Most of commercial codes simulate the turbulent boundary layer by using standard eddy viscosity models. These models 
cannot capture the non-equilibrium effects in the adverse pressure gradient [31]. Thus, using any turbulence modeling for the problems with the occurrence of separation and in the adverse pressure gradient could be challenge.

In this research, the flow separation around the ducts without fans has two following effects on the lift and drag:

1- Reduction in the lift happens after possible stall and flow separation around symmetrical duct at $A o A=20^{\circ}$ (see Figure A. 1a): The $k-\omega$ turbulence modeling predicted that at the same AoA but its calculated lift was much lower than the experimental result. The $k-\varepsilon$ turbulence modeling predicted stall at $A o A=30^{\circ}$ and its calculated lift is very closer to the experimental results.

2- Increment in the drag after flow separation: Both $k-\omega$ and $k-\varepsilon$ turbulence modeling showed increment in the drag after occurrence of the flow separation (see Figure A. 1b). Both method computed drag lower than the experimental values. But as it seen, the $k-\varepsilon$ turbulence modeling gave closer results to the experimental values.

Although there is no unique turbulence modeling for all CFD problems, each model can solve some specific problems accurately. In this research, using the $k-\varepsilon$ turbulence modeling showed better results than the other methods. It may not predict the exact location and the effects of the flow separation, which is generally the weakness of most of turbulence modeling, but the overall results are closer to the experiments.

\section{Wall function for wall-bounded turbulent flow}

Turbulent flow will be affected by the presence of walls due to the viscosity of the fluid and changes in the mean velocity field close to the wall. The near wall region can be divided into three 
sublayers. In the laminar or viscous sublayer, which is attached to the wall, the molecular viscosity plays the main role in momentum, heath, and mass transfer. In the outer sublayer, named fully turbulent layer, turbulence plays the main role. Between these two sublayer, the buffer layer is another sublayer with the mixed effects of molecular viscosity and turbulence [21].

$Y^{+}$and $Y^{*}$ are two dimensionless distance from the wall. These parameters are good scales for finding sublayers near the wall. The latest version of ANSYS-Fluent software uses $Y^{*}$ for this measurement. In the ANSYS-Fluent and by using standard $k-\varepsilon$ and the RNG $k-\varepsilon$ turbulence modeling, the standard wall functions are set as default functions for modeling near-wall region. In this region, the log-law is employed when $Y^{*}>11.225$. Also the laminar stress-strain relationship is used when $Y^{*}<11.225[21]$.

In this research, the $Y^{*}$ was measured for different simulations (ducted fans and ducts without fans in different AoAs). The maximum value for $Y^{*}$ was in the range of 300 in all AoAs and the standard wall function showed acceptable results for wide range of AoAs. The only large differences between experimental and CFD results are when $80^{\circ}<A o A<100^{\circ}$ for the modeling of horizontal forces (drag and $F_{x}$ ). Finally, using standard $k-\varepsilon$ and the RNG $k-\varepsilon$ turbulence modeling combined with the standard wall functions gave reasonable results in this research. 


\section{Appendix B: Geometry of meshes}

\section{Geometry of meshes for symmetrical and asymmetrical ducts without fan}

The external body of symmetrical duct was created by revolving the upper side of NACA 0012 airfoil, as shown in Figure 3-3.

The external body of asymmetrical duct consists of different profiles of high lift FX 63-137 airfoil, as shown in Figure 3-4. The upper side profile of FX 63-137 airfoil is applied at $120^{\circ}$ on the upper side of the asymmetrical duct. Also the lower side profile of FX 63-137 airfoil is applied at $120^{\circ}$ on the lower side of the asymmetrical duct. There are two similar smooth transitional regions on the left and right sides of the duct to connect lower part to upper part.

The internal bodies for both symmetrical and asymmetrical ducts are similar, as shown in Figure 3-3b and Figure 3-4d. To reduce the number of parameters which have effects on this research, the mean diameters at the duct inlet/outlet are set equal to the duct chord length $(d=c)$ for both symmetrical and asymmetrical ducts, as shown in Figure 3-3b and Figure 3-4d.

The grid generation for all meshes have been done using GAMBIT software. The 3-D meshes for ducts without fans were created by assuming $c=d=1$ as characteristic length. Then a halfcircle with radius of $r=10$ was assumed and revolved about the $\mathrm{x}$-axis to create a sphere surface as outer boundary. The selected models are tilting about the $z$-axis and as a result the $x y$ plane is the plane of symmetry for simulations.

The solid external and internal faces of ducts and spherical surface of the outer boundary consist of triangular cells. The volume between outer boundary and duct consists of tetrahedron cells with triangular faces. These volumetric cells are very fine around the duct's surfaces and become coarse as they go farther from ducts. 
Table B. 1 shows the number of spacing and interval size for different edges of the meshes for symmetrical and asymmetrical ducts.

Table B. 1: Number of spacing and interval size for different edges of the meshes for symmetrical and asymmetrical ducts

\begin{tabular}{|l|c|}
\hline \multicolumn{1}{|c|}{ Edge location } & No. of spacing and interval size \\
\hline Outer edge for external body of duct & 100 , equal size \\
\hline Inner edge for internal body of duct & 100, equal size \\
\hline Duct's inlet circular edge (at leading edge) & 300, equal size \\
\hline Duct's outlet circular edge (at trailing edge) & 300, equal size \\
\hline Half-circle edge of spherical boundary & 50, equal size \\
\hline
\end{tabular}

The types of surface boundaries are "Wall" for duct's internal and external faces and "Inlet Velocity" for spherical inlet face. The number of volumetric cells for ducts without fan are shown in Table B. 3 .

\section{Geometry of meshes for symmetrical and asymmetrical ducted fans}

The outer boundary and ducts specifications of these meshes are similar to meshes for ducts without fans. Adding rotor and electromotor (or shaft) to the models needs to define proper spacing and interval size for these components. Table B. 2 shows the number of spacing and interval size for different edges of rotor and shaft for two ducted fans.

Table B. 2: Number of spacing and interval size for different edges of additional components (rotor and shaft)

\begin{tabular}{|l|c|}
\hline \multicolumn{1}{|c|}{ Edge location } & No. of spacing and interval size \\
\hline Outer edge for rotor & 300, equal size \\
\hline Central edge for rotor to connect to the shaft & 24, equal size \\
\hline Radial direction on the rotor plane & 60 , equal size \\
\hline Thickness of the rotor & 3 , equal size \\
\hline Circular edge of shaft's cross section & 24, equal size \\
\hline Head of the shaft & 6, equal size \\
\hline Central body of the shaft & 19, equal size \\
\hline End part of the shaft & 16, equal size \\
\hline
\end{tabular}


The types of surface boundaries for these additional components are "Wall" for rotor tip and shaft's body faces and "Inlet Velocity" for rotor inlet and outlet faces. The number of volumetric cells for ducted fans are also presented in Table B. 3.

Table B. 3: Number of volumetric tetrahedron cells for different models of ducts with/without fans

\begin{tabular}{|l|c|}
\hline \multicolumn{1}{|c|}{ Model } & No. of volumetric cells \\
\hline Symmetrical duct without fan/rotor & 849,339 \\
\hline Asymmetrical duct without fan/rotor & 830,982 \\
\hline Symmetrical ducted fan & $1,068,942$ \\
\hline Asymmetrical ducted fan & $1,142,536$ \\
\hline
\end{tabular}




\section{Appendix C: Designing, producing, and preparing actual models of ducted fans}

The actual models of ducted fans were designed by using CATIA 5 software in the "Computer Lab" of Ryerson University. The designs were the same as the CFD models by Gambit software, but the generated "stl" files could be used by a 3D printer to produce the models from the "Ivory ABS Sparse Material". The designed models are shown in Figure C. 1.

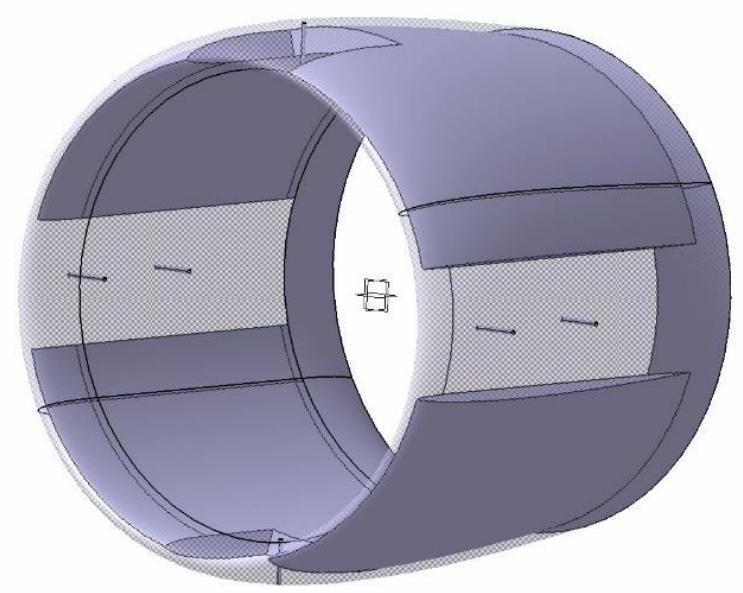

(a)

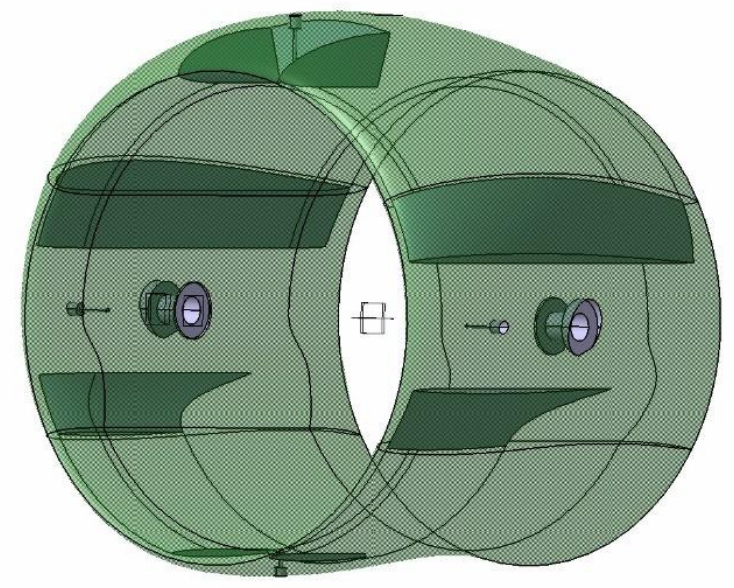

(b)

Figure C. 1: Designs of models by CATIA 5; (a) Symmetrical duct, (b) Asymmetrical duct Next, these models were printed by "Dimension sst 1200es" 3D printer. The printing process took 48 hours for each duct. Then, each printed duct had a hand smoothing process to reduce their skin frictions. Figure C. 2 shows asymmetrical duct with rough surface. 


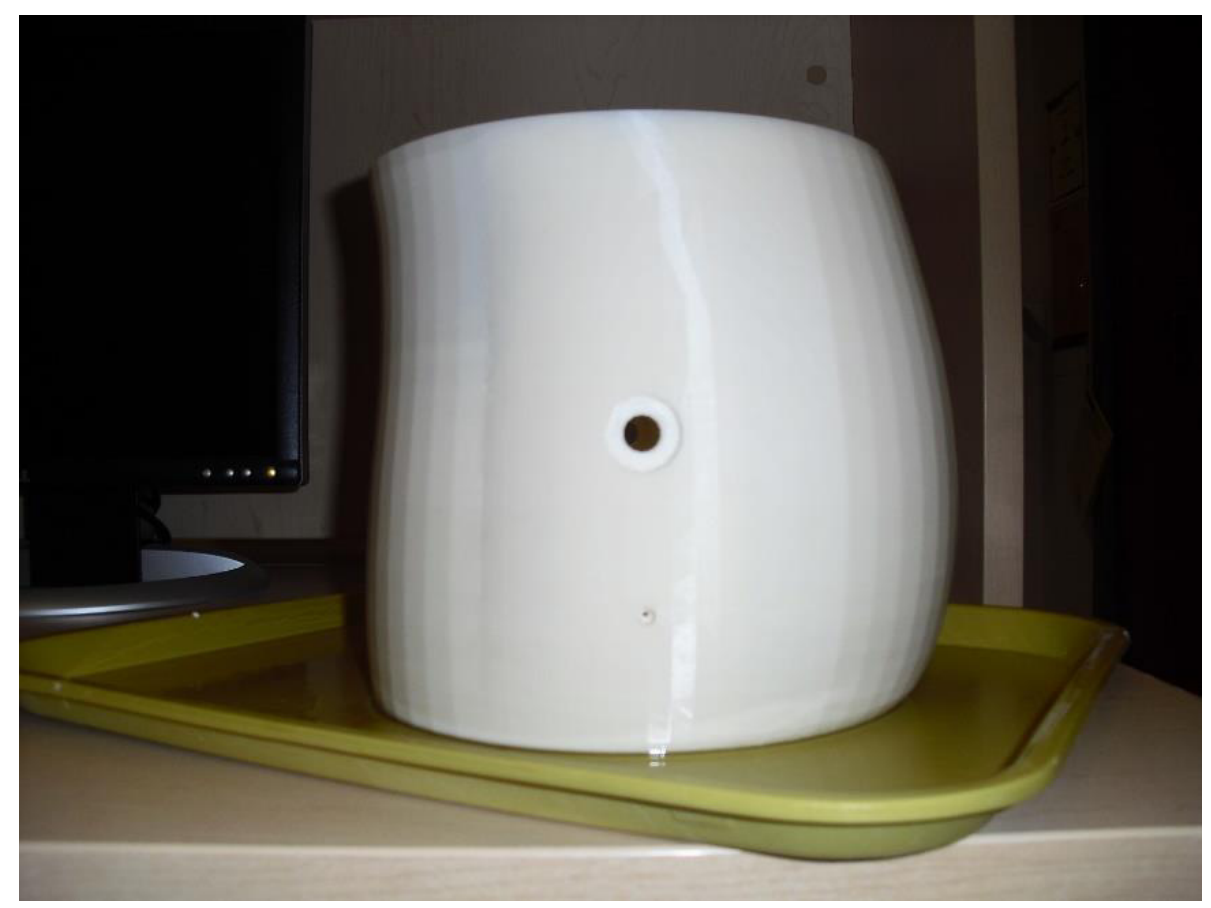

Figure C. 2: Asymmetrical duct before smoothing the surface

These ducts were equipped with electromotor and 3-bladed propeller as shown in Figure C. 3.

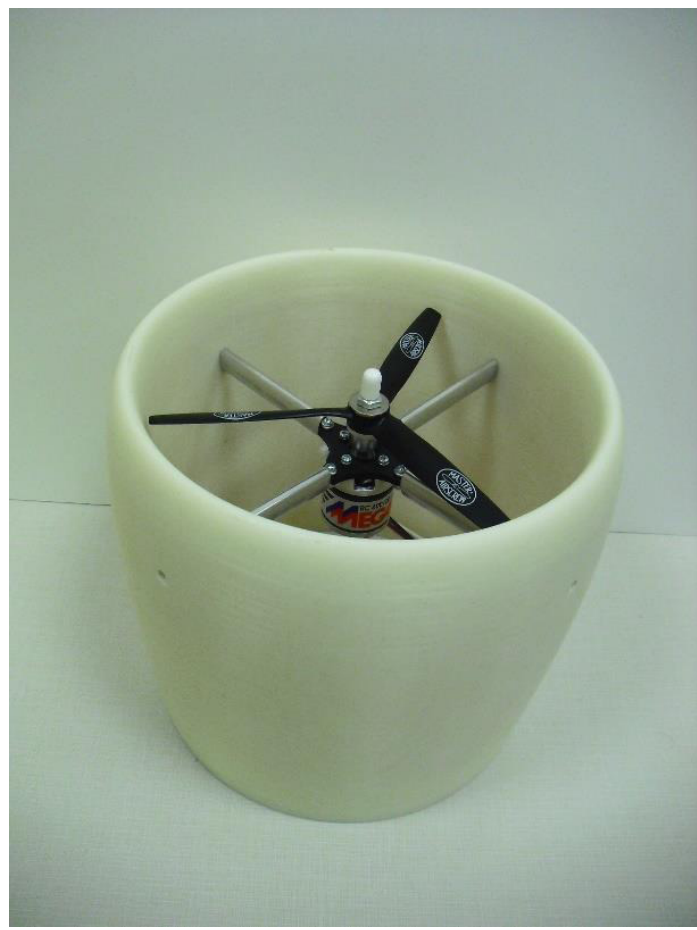

(a)

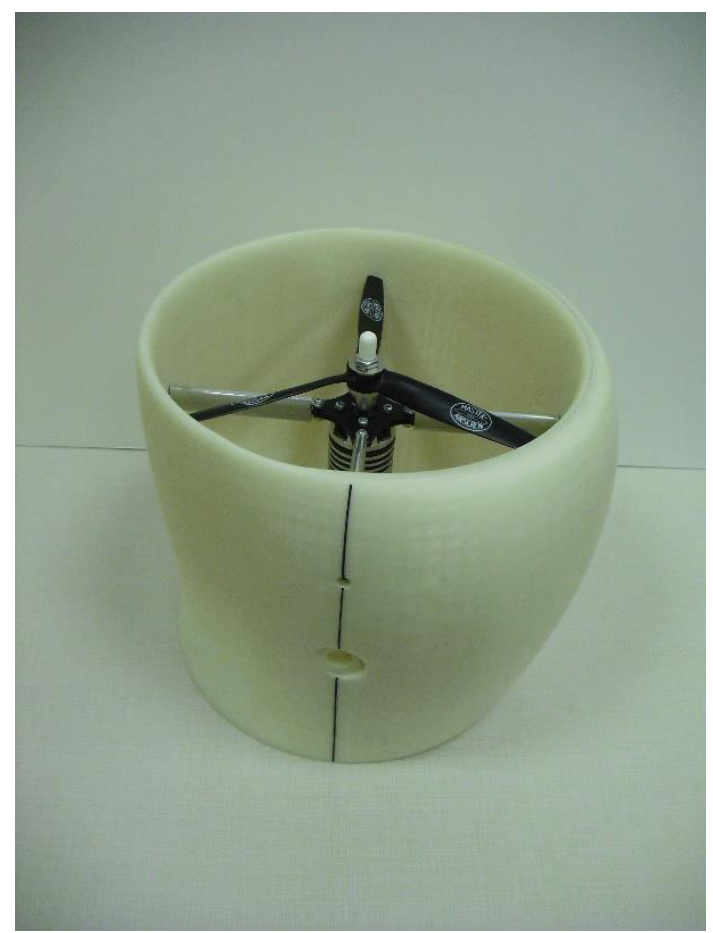

(b)

Figure C. 3: Two ducts after installation of electromotor and 8x6" 3-bladed propeller: (a) symmetrical ducted fan (b) asymmetrical ducted fan 
The supporting frame was designed for attaching ducts to it, providing tilting motion for ducts through a gear system, and measuring AoA by a $360^{\circ}$ protractor as shown in Figure C. 4.

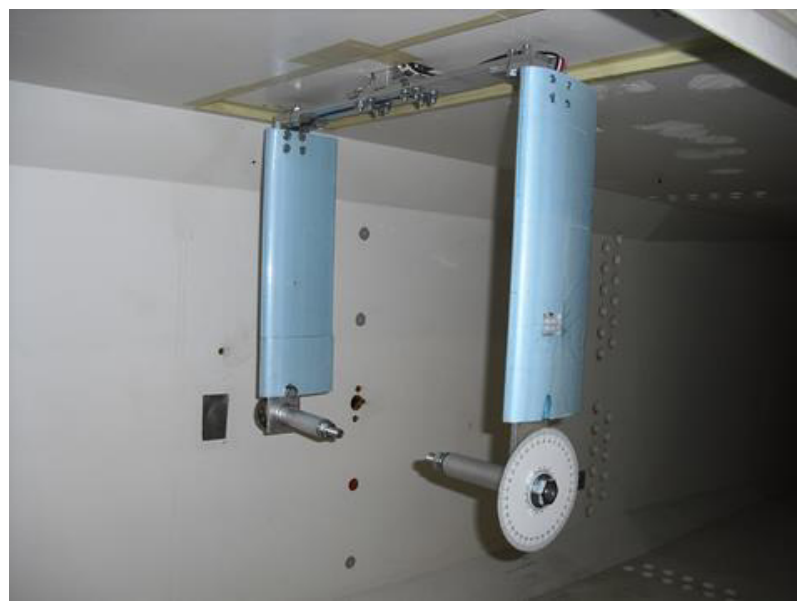

(a)

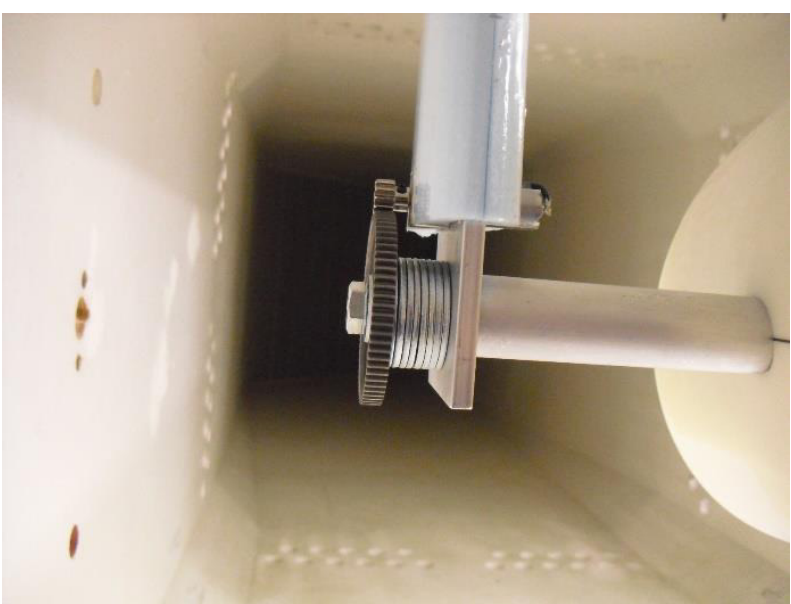

(b)

Figure C. 4: (a) Supporting frame, and (b) Gear system for tilting ducts

There are some devices to control the tilting motion of ducts. The gear system consists of an electromotor (shawn in Figure C. 4b) which needs 6 Volt power source, and a control unit to control the tilting rate (shawn in Figure C. 5).

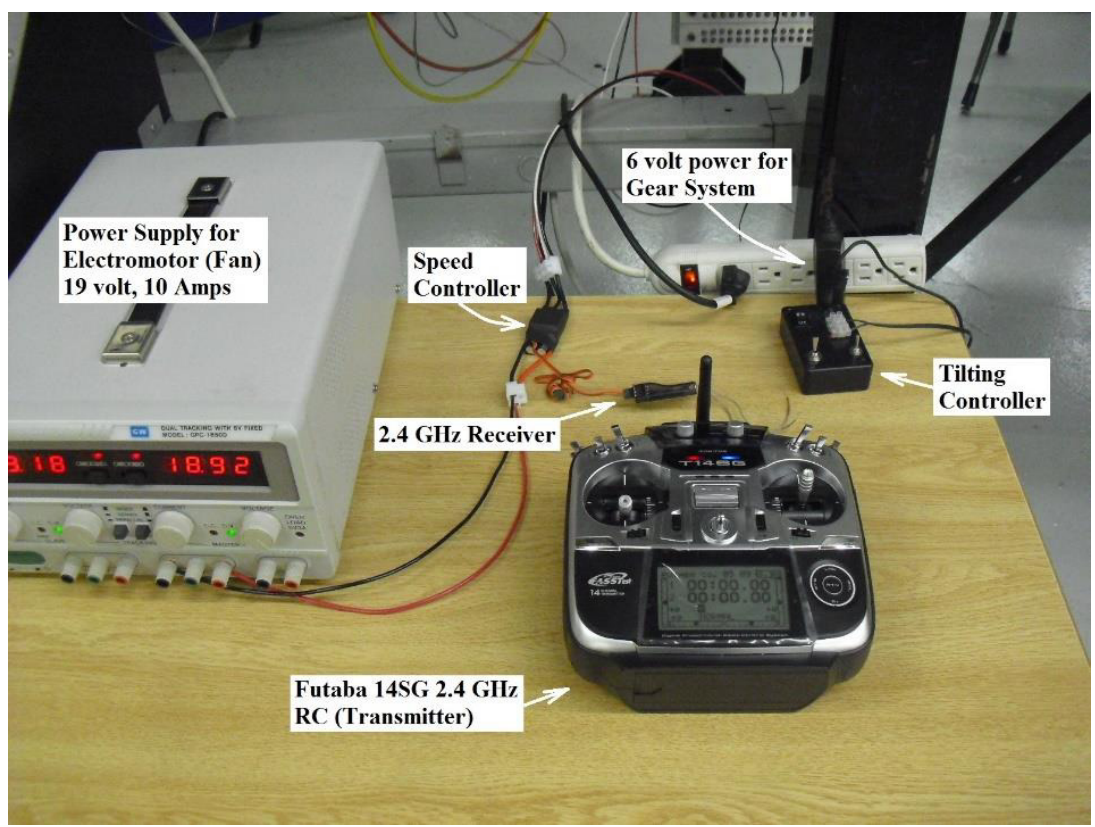

Figure C. 5: Devices for controlling propeller's RPM and duct's tilting motion 
The rotational speed of propeller can be controlled by a Futaba $2.4 \mathrm{GHz}$ transmitter and receiver which is connected to the speed controller. The power supply provides 19 volts and 10 Amps current to the speed controller. All these components are shown in Figure C. 5. The speed controller has been installed inside the wind tunnel test section to prevent it from excessive heath during tests as shown in Figure C. 6.

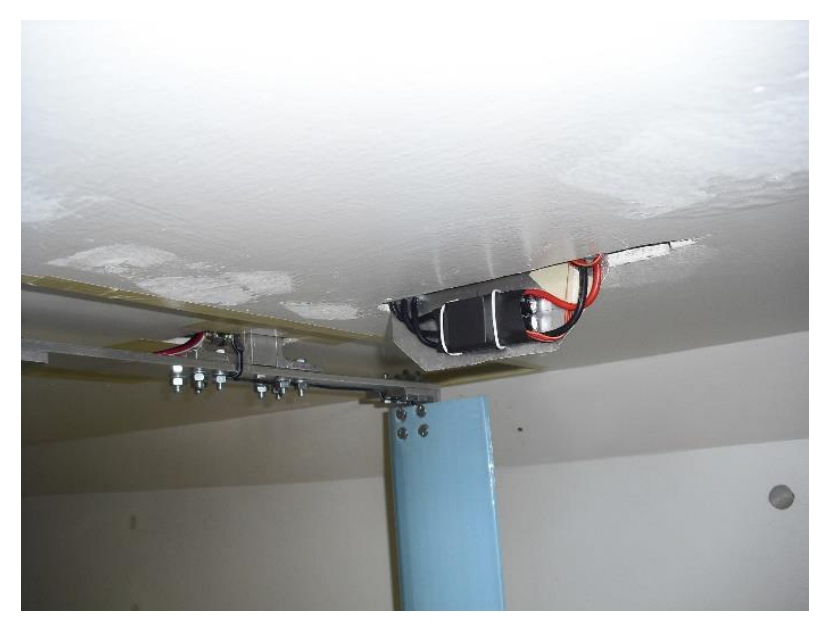

Figure C. 6: Installation of speed controller in the test section at the back of supporting frame 


\section{Appendix D: Wind tunnel facility}

The large wind tunnel is a closed loop wind tunnel and consists of different major sections. The main sections are listed as following:

1- The Test section, which is the location for hanging the models for tests (Figure D. 1a)

2- The closed loop tunnel

3- The motor and Fan

4- The main control consul (Figure D. 1b)

5- The manometers for calculating the speed of air inside the tunnel (Figure D. 2)

6- The balancing system for measuring forces and pitching moment on the model (Figure D.

3)

The test section cross area is $36 \times 36$ inch and has a large length which gives a very good visibility on the model.

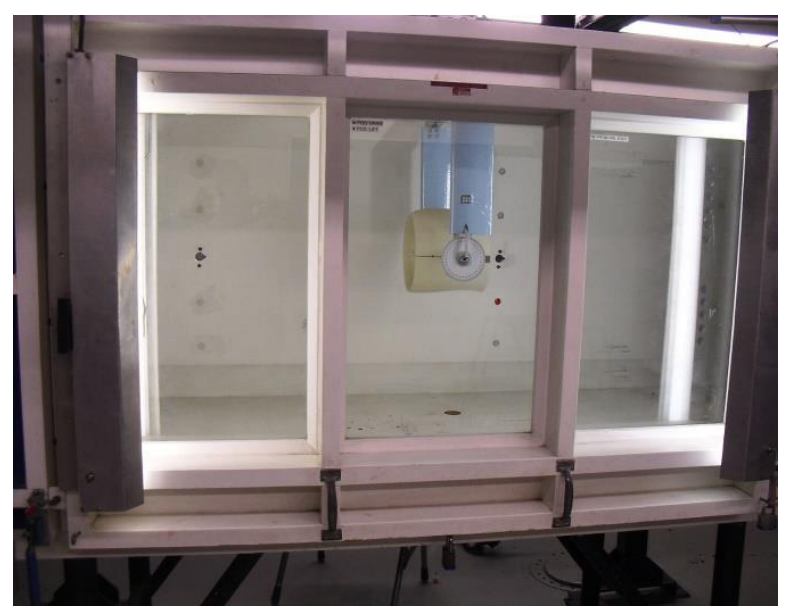

(a)

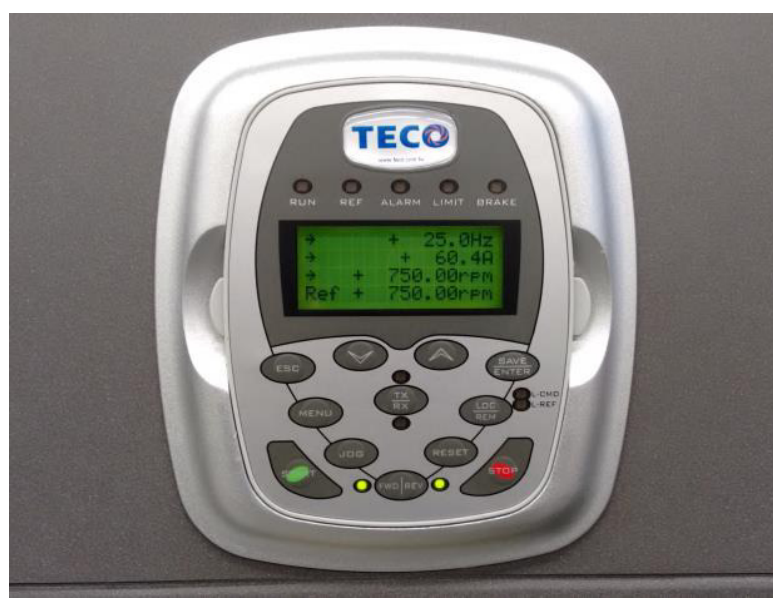

(b)

Figure D. 1: (a) The "Test Section" and (b) the main control unit 


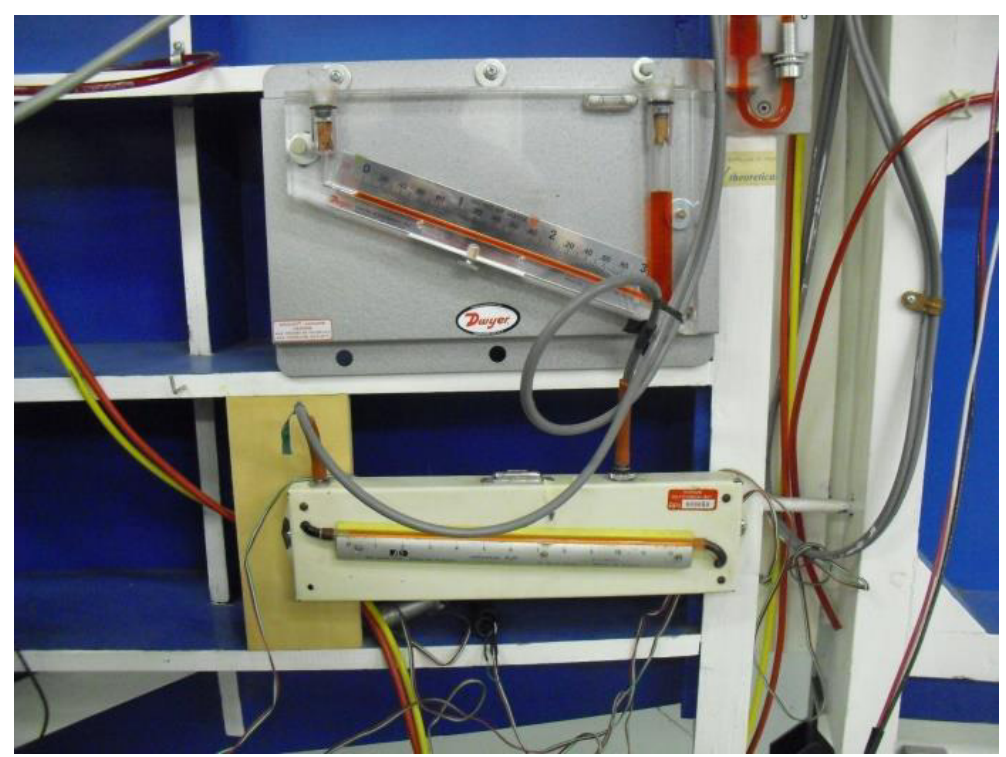

Figure D. 2: The manometers for measuring air velocity in the test section

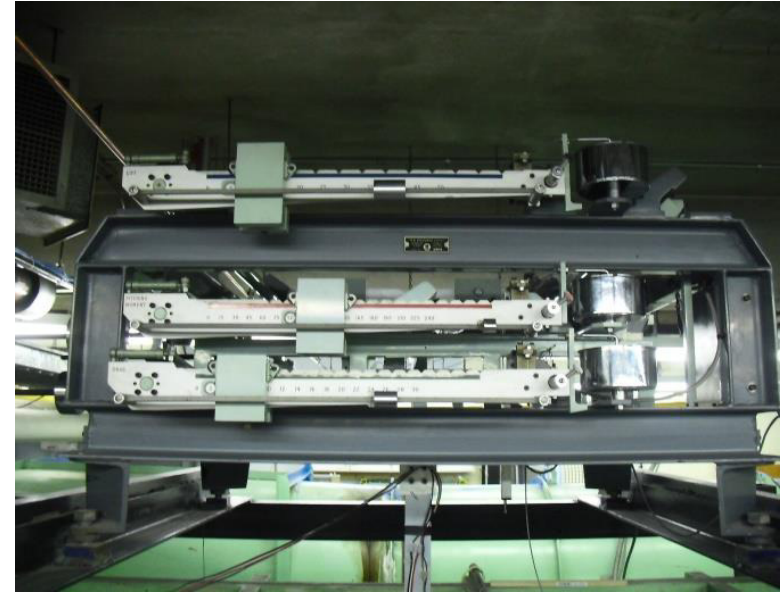

(a)

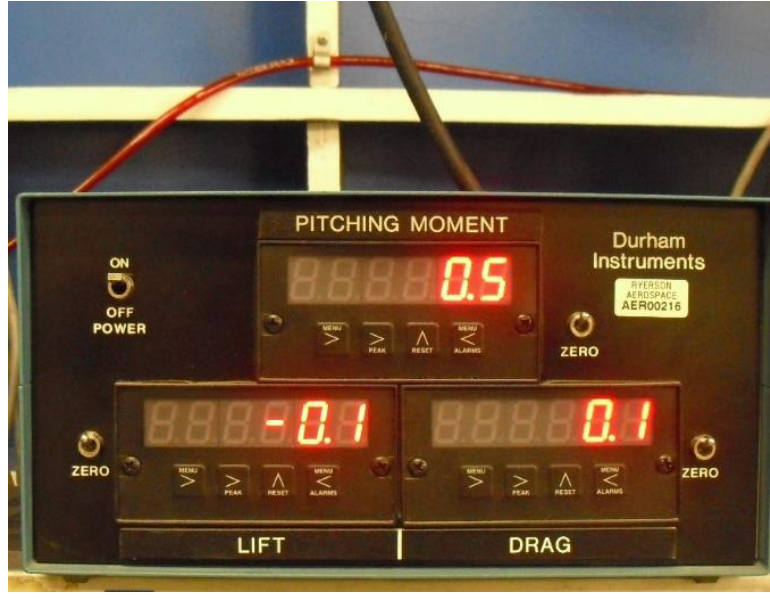

(b)

Figure D. 3: (a) The balancing device for hanging the model and measuring forces and pitching moments, (b) the display for measured forces and pitching moment 


\section{Appendix E: Measuring aerodynamic forces on the frame and ducts with/without fans}

All models tested consist of two major components: ducted fans (electromotor + 8"x6" 3-bladed propeller + connecting rods + ducts) and supporting frame (aluminum supports + tilting gear motor $+360^{\circ}$ protractor) to connect ducts to the load measuring system.

The protractor provided good reading of the AoA with the error of reading less than 0.5 degree. The flow velocity inside the test section of the wind tunnel can be found by calculating the dynamic pressure of the flow. There is a pitot tube inside the test section which is connected to a manometer outside the test section. By reading $h$ on the manometer (inch of water with accuracy of \pm 0.01 ) and using the following equations the flow velocity in the test section can be found:

$$
q_{\infty}=\frac{1}{2} \rho_{\text {air }} V^{2}=h \rho_{\text {water }} g
$$

Then:

$$
V=\sqrt{\frac{2 h \rho_{\text {water }} g}{\rho_{\text {air }}}}
$$

The air density can be found by knowing ambient pressure and temperature and using the following equation:

$$
\rho_{a i r}=\frac{p_{a m b}}{R T_{a m b}}
$$

As an example, in the test in June 16, 2014, the following readings were done in a test:

$$
\begin{aligned}
& T_{a m b}=81^{\circ} \mathrm{F}=27.22^{\circ} \mathrm{C}=300.37 \mathrm{~K} \\
& p_{a m b}=101,800 \mathrm{~Pa} \\
& R=287.056 \frac{\mathrm{Jol}}{\mathrm{kg} \mathrm{K}}
\end{aligned}
$$

And the air density is: $\rho_{\text {air }}=1.181 \mathrm{~kg} / \mathrm{m}^{3}$

$$
h=0.52 \text { inch of water }=0.013208 \text { m of water }
$$


$\rho_{\text {water }}=1000 \mathrm{~kg} / \mathrm{m}^{3}$

$g=9.81 \mathrm{~m} / \mathrm{s}^{2}$

and then: $V=14.813 \mathrm{~m} / \mathrm{s}$

The load measuring system measures horizontal and vertical components $\left(F_{x}\right.$ and $\left.F_{y}\right)$ and also pitching moment $\left(M_{z}\right)$ of pressure forces acting on the models. The measuring system could measure forces by $0.1 \mathrm{lb}$ and pitching moments by $0.1 \mathrm{lb}$. in scales. The readings error for forces and pitching moments was $\pm 0.05 \mathrm{lb}$ and $\pm 0.05 \mathrm{lb}$. in respectively. The proper conversion factors were used to convert English units ( $l b$ and $l b . i n)$ to SI units ( $N$ and $N . m$ ).

The lift and drag for frame individually were measured as $L_{\text {frame }}=0$ and $D_{\text {frame }}=2.4606 \mathrm{~N}$ before testing the models. Later, the measured $D_{\text {frame }}$ is used in the proposed method for calculating the inlet flow velocity to the ducted fans.

The $F_{y}, F_{x}$, and $M_{z}$ (lift, drag, and pitching moment) for symmetric and asymmetric ducts without fans were measured in the range of $-30^{\circ} \leq \alpha \leq 120^{\circ}$ (with $10^{\circ}$ increment in AoA) in the first step. Next, both symmetric and asymmetric ducts were equipped with electromotor and propellers as shown in Figure 4-15 and tested in the wind tunnel. The $F_{y}, F_{x}$, and $M_{z}$ for symmetric and asymmetric ducted fans were measured for the same range of AoAs. The wind tunnel velocity was set to $U=15 \mathrm{~m} / \mathrm{s}$ in all tests and each test was repeated three times to have accurate data. At the beginning of each test for ducted fans at $A o A=0^{\circ}$, the electric power to the electromotor was set to have the condition of $\Sigma F_{x}=0$ (similar to cruise mode for steady level flight of proposed VTOL UAV) as follows:

$$
\Sigma F_{x}=D_{\text {frame }}+D_{\text {duct }}-T=0 \quad \text { (only at zero AoA) }
$$


The power to the electromotor was constant during all tests. Horizontal and vertical forces and also pitching moments were recorded in the range of $-30^{\circ} \leq \alpha \leq 120^{\circ}$ (with $10^{\circ}$ increment in AoA) for both ducted fans. 


\section{Appendix F: Measuring pitching moments of the ducts' weights}

The centre of gravity for both symmetrical and asymmetrical ducts are not located on the tilting axis. Then, their weights generate pitching moments about tilting axis. The pitching moments due to ducts' weight were measured for both symmetrical and asymmetrical ducts with/without fans during experiments. Then, these pitching moments were subtracted from the gross measured pitching moments to obtain the net aerodynamic pitching moment in different AoAs for each duct.

\section{Ducts without fans}

Both ducts were printed from "Ivory ABS Sparse Material" with density of $1.06 \mathrm{gr} / \mathrm{cm}^{3}$. The mass of symmetrical duct is $1.437 \mathrm{~kg}$ and its weight is $14.092 \mathrm{~N}$. The mass of asymmetrical duct is $1.306 \mathrm{~kg}$ and its weight is $12.808 \mathrm{~N}$. The pitching moments due to the weight of each duct in different AoAs were measured during tests, as shown in Figure F. 1. The centre of gravity of symmetrical duct is located on the line of symmetry and maximum pitching moment due to its weight is $-0.1356 \mathrm{~N} . \mathrm{m}$, which occurs at $A o A=0^{\circ}$ (or $0.1356 \mathrm{~N} . \mathrm{m}$ at $A o A=180^{\circ}$ ). The centre of gravity of asymmetrical duct is above the line of symmetry and maximum pitching moment due to its weight is $-0.3446 \mathrm{~N} . \mathrm{m}$, which occurs at $A o A=-50^{\circ}$ (or $0.3446 \mathrm{~N} . \mathrm{m}$ at $A o A=130^{\circ}$ ).

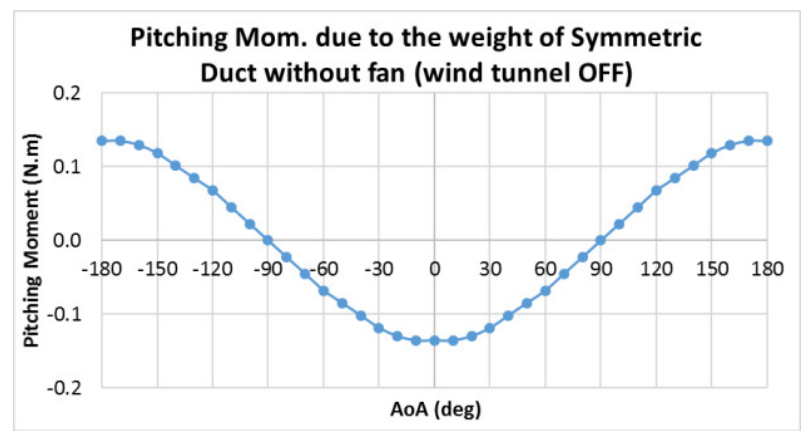

(a)

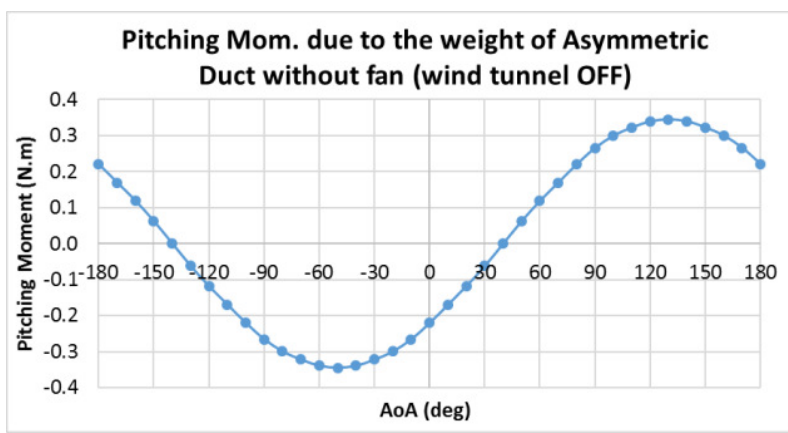

(b)

Figure F. 1: Pitching moments due to the weight of ducts without fans; (a) symmetrical duct, (b) asymmetrical duct 


\section{Ducted fans}

Both ducts were equipped with electromotor, propeller and connection rods, as shown in Figure 4-15. Same as the previous step, the pitching moments due to the weight of each ducted fan in different AoAs were measured during tests, as shown in Figure F. 2. The centre of gravity of symmetrical ducted fan is located on the line of symmetry and maximum pitching moment due to its weight is -0.2260 N.m, which occurs at $A o A=0^{\circ}$ (or 0.2260 N.m at $A o A=180^{\circ}$ ). The centre of gravity of asymmetrical ducted fan is above the line of symmetry and maximum pitching moment due to its weight is -0.4067 N.m, which occurs at $A o A=-40^{\circ}$ (or 0.4067 N.m at $A o A=$ $\left.140^{\circ}\right)$

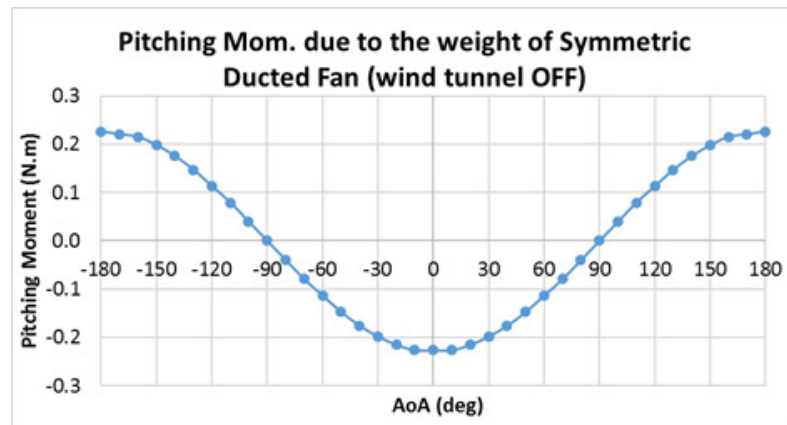

(a)

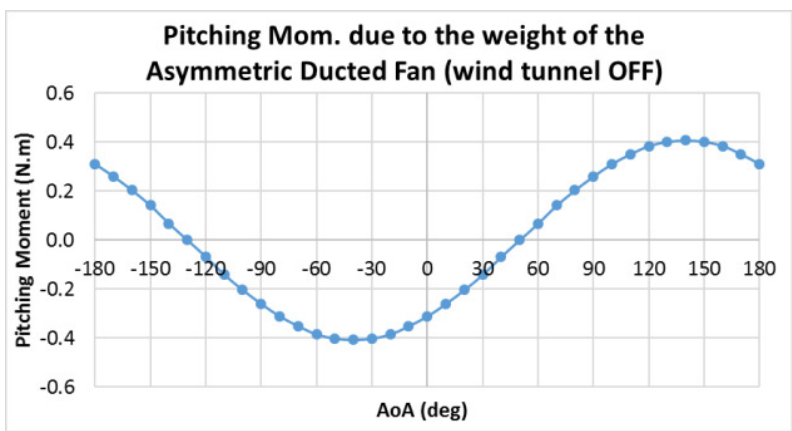

(b)

Figure F. 2: Pitching moments due to the weight of ducted fans; (a) symmetrical ducted fan, (b) asymmetrical ducted fan 


\section{Appendix G: Error analysis for measuring aerodynamic coefficients}

The error of reading of quantities during any experiment should be clarified to show the accuracy of the measurements. Two sources of errors for measuring aerodynamic coefficients of tilting ducts in the wind tunnel were reading of loads (lift, drag and pitching moment) and $h$ of the manometer. The accuracy for reading these quantities are shown in the Table G. 1 .

Table G. 1: Error of reading for different quantities during experiments in the wind tunnel

\begin{tabular}{|l|c|}
\hline \multicolumn{1}{|c|}{ Quantity } & Error of reading \\
\hline Lift and Drag & $\pm 0.05 \mathrm{lb}$ \\
\hline Pitching Moment & $\pm 0.05 \mathrm{lb}$. in \\
\hline$h$ of the manometer & \pm 0.01 inch of water \\
\hline
\end{tabular}

The effects of the error during reading these quantities on the calculation of aerodynamic coefficients can be described by using equations (3.42), (3.43), (3.44), and (E.2). For example, for error on the lift calculation:

$$
L=\frac{1}{2} \rho S V^{2} C_{L}=\frac{1}{2} \rho_{\text {air }} S C_{L} \frac{2 h \rho_{\text {water }} g}{\rho_{\text {air }}}=S C_{L} h \rho_{\text {water }} g
$$

Then:

$$
C_{L}=\frac{1}{S \rho_{\text {water }} g} \frac{L}{h}
$$

As the surface area of ducts (S), the density of water $\left(\rho_{\text {water }}\right)$ and acceleration of gravity $(\mathrm{g})$ are constant values, then lift coefficient is the function of two other measured parameters:

$$
C_{L}=f(L, h)
$$

The error analysis for the similar cases is given by Taylor [32] as following:

$$
\begin{aligned}
& \text { If } z=C \frac{x}{y},(C \text { is a constant }) \text {, then: } \\
& \frac{\Delta z}{z}=\left[\left(\frac{\Delta x}{x}\right)^{2}+\left(\frac{\Delta y}{y}\right)^{2}\right]^{1 / 2}
\end{aligned}
$$

And in similar way, the error in the $C_{L}, C_{D}$, and $C_{M}$ can be found as: 


$$
\begin{aligned}
& \frac{\Delta C_{L}}{C_{L}}=\left[\left(\frac{\Delta L}{L}\right)^{2}+\left(\frac{\Delta h}{h}\right)^{2}\right]^{1 / 2} \\
& \frac{\Delta C_{D}}{C_{D}}=\left[\left(\frac{\Delta D}{D}\right)^{2}+\left(\frac{\Delta h}{h}\right)^{2}\right]^{1 / 2} \\
& \frac{\Delta C_{M}}{C_{M}}=\left[\left(\frac{\Delta M}{M}\right)^{2}+\left(\frac{\Delta h}{h}\right)^{2}\right]^{1 / 2}
\end{aligned}
$$

Results from equations (G.4) and (G.6) for symmetrical duct show large errors if $A o A \approx 0^{\circ}$, as there is no lift or pitching moment for symmetrical duct at $A o A \approx 0^{\circ}$ in the experiments. Then, the maximum errors for each aerodynamic coefficient can be found by using the following equations:

$$
\begin{aligned}
& \Delta C_{L}=C_{L}\left[\left(\frac{\Delta L}{L}\right)^{2}+\left(\frac{\Delta h}{h}\right)^{2}\right]^{1 / 2} \\
& \Delta C_{D}=C_{D}\left[\left(\frac{\Delta D}{D}\right)^{2}+\left(\frac{\Delta h}{h}\right)^{2}\right]^{1 / 2} \\
& \Delta C_{M}=C_{M}\left[\left(\frac{\Delta M}{M}\right)^{2}+\left(\frac{\Delta h}{h}\right)^{2}\right]^{1 / 2}
\end{aligned}
$$

The maximum error for each aerodynamic coefficient was calculated and shown in the Table G. 2 based on the measurements of aerodynamic forces and pitching moments $(L, D, M)$ and the height of the water in the manometer $(h)$ in each experiment in the wind tunnel.

Table G. 2: Maximum error for each aerodynamic coefficient in the different experiment

\begin{tabular}{|l|c|c|c|}
\hline \multicolumn{1}{|c|}{ Type of duct } & $C_{L}$ (Max. error) & $C_{D}$ (Max. error) & $C_{M}$ (Max. error) \\
\hline Symmetrical duct without fan & \pm 0.011812 & \pm 0.013078 & \pm 0.002381 \\
\hline Asymmetrical duct without fan & \pm 0.014203 & \pm 0.015041 & \pm 0.002603 \\
\hline Symmetrical ducted fan & \pm 0.014009 & \pm 0.012223 & \pm 0.001729 \\
\hline Asymmetrical ducted fan & \pm 0.024605 & \pm 0.016228 & \pm 0.002589 \\
\hline
\end{tabular}

These errors were applied to the experimental aerodynamic coefficients of symmetrical and asymmetrical ducts with/without fans and the limits of possible errors are compared and shown in the Figure G. 1 and Figure G. 2. As shown, the possible errors from measurements have more effects on the drag coefficients and less effects on the pitching moments of the models. 


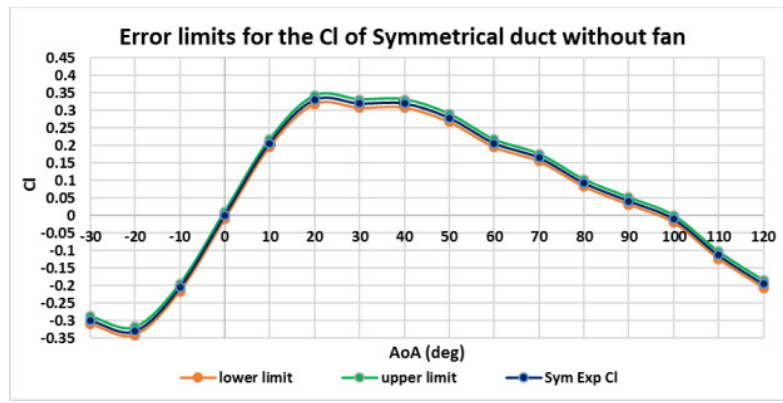

(a)

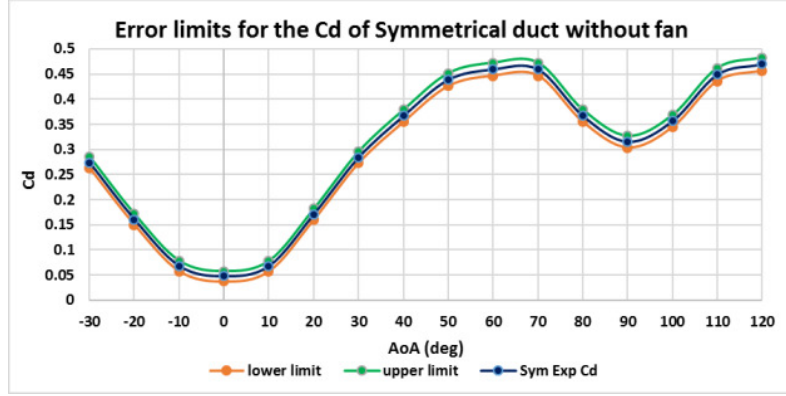

(b)

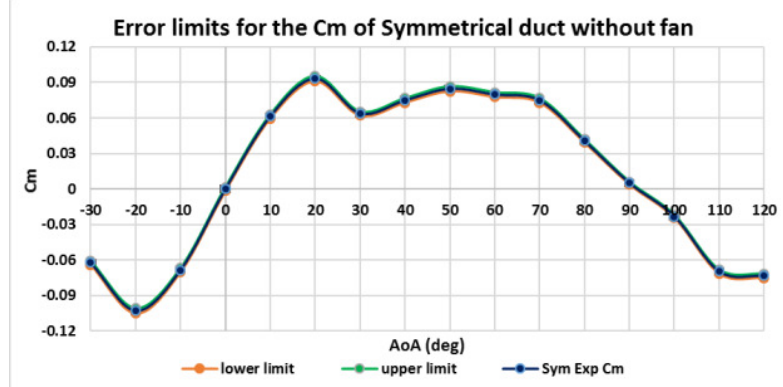

(c)

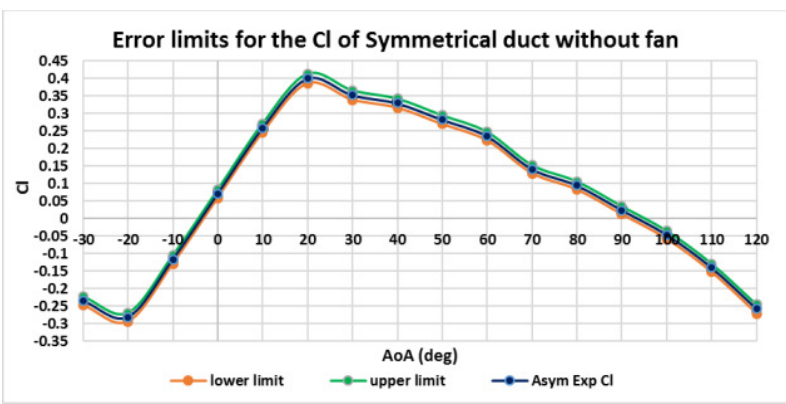

(d)

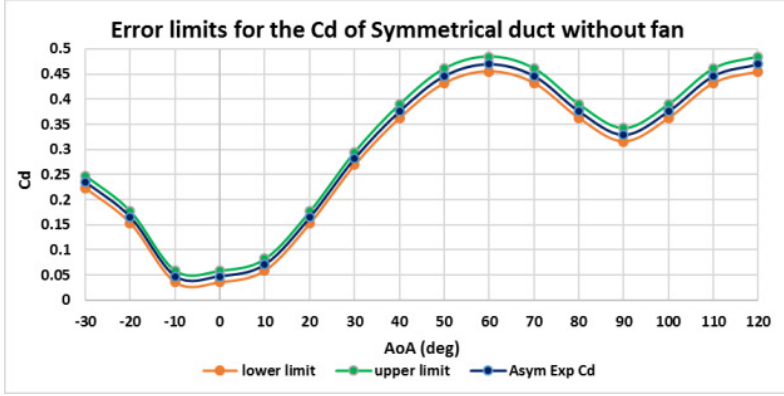

(e)

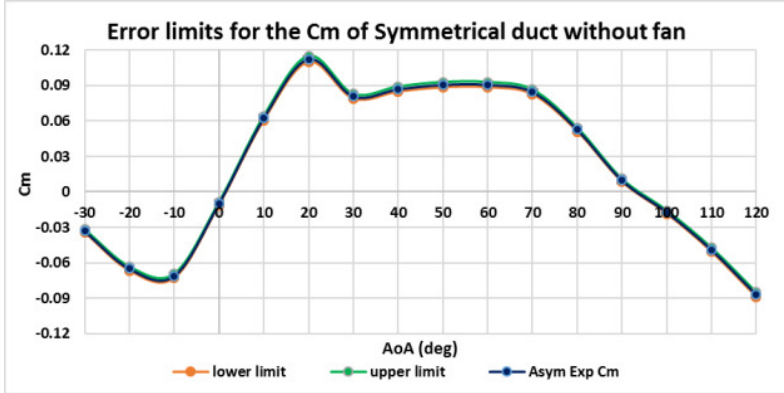

(f)

Figure G. 1: Error limits for experimental aerodynamic coefficients of ducts without fans; (a), (b), and (c) are error limits on the $C_{l}, C_{d}$, and $C_{m}$ of symmetrical duc, (d), (e), and (f) are error limits on the $C_{l}, C_{d}$, and $C_{m}$ of asymmetrical duct. 


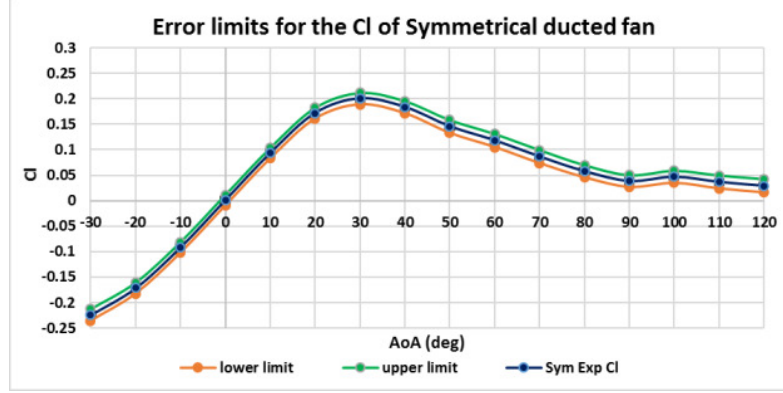

(a)

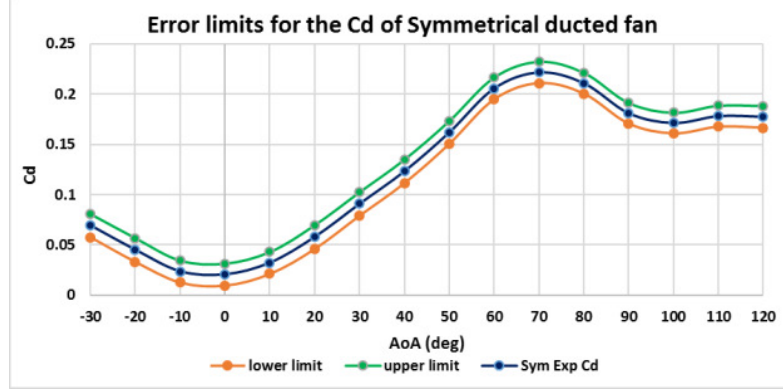

(b)

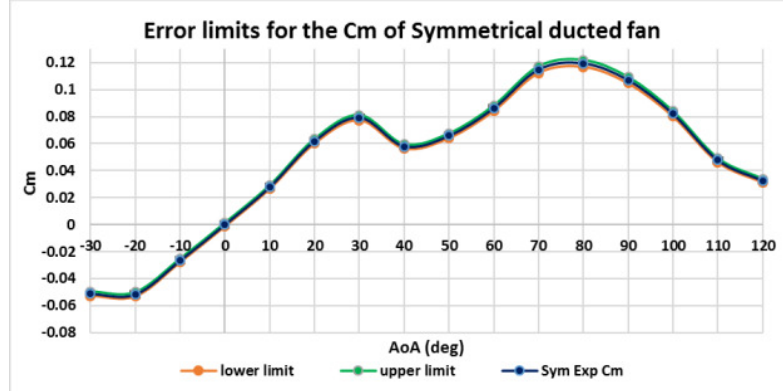

(c)

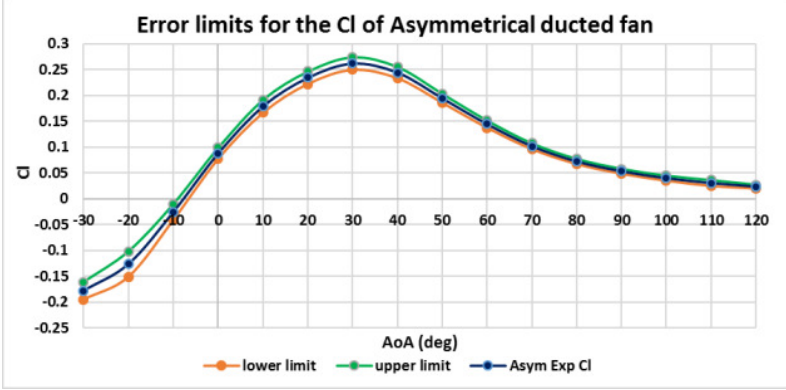

(d)

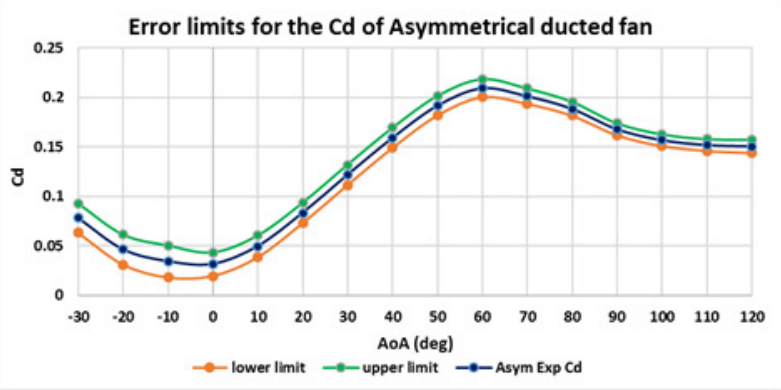

(e)

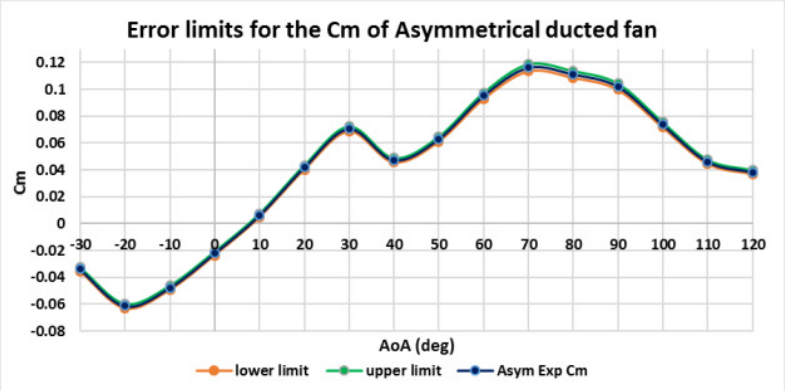

(f)

Figure G. 2: Error limits for experimental aerodynamic coefficients of ducted fans; (a), (b), and (c) are error limits on the $C_{l}, C_{d}$, and $C_{m}$ of symmetrical ducted fan, (d), (e), and (f) are error limits on the $C_{l}, C_{d}$, and $C_{m}$ of asymmetrical ducted fan.

The error analysis shows the accuracy of the measured quantities in the experiments are acceptable and can be used for comparing with the CFD results. 


\section{Appendix H: Summary of ANSYS-Fluent settings in the transient mode and User Defined Functions}

\section{Summary of ANSYS-Fluent settings in transient mode}

To complete all simulations during this project, ANSYS-Fluent has been used nearly 200 times. Entering the right amounts for parameters and choosing the right setting for software can save time and give the right answers for each simulation. This will be more important if users find out it takes more than 24 hours to complete one transient simulation. To start ANSYS-Fluent in "Transient Mode", both mesh and UDF files must be in the same working directory. The final results (cl-history, cd-history and cm-history) will be saved in the same working directory.

The following are steps for running ANSYS-Fluent to find $C_{l}, C_{d}$ and $C_{m}$ of symmetrical ducted fan in transition condition, when ducted fan has tilting motion $\left(\omega=10 \mathrm{deg} / \mathrm{sec}, \alpha_{\min }=-30^{\circ}\right.$ and $\alpha_{\max }=120^{\circ}$ ). By using UDFs, the tilting angle and the velocity of the inlet flow to the rotor plane are being changed automatically based on the running time.

By choosing time steps as $\Delta t=0.01 \mathrm{sec}$, the total number of 1800 time steps are needed for simulating $18 \mathrm{sec}$ transient mode for tilting the symmetrical ducted fan. Adding maximum 100 iterations per time step will increase the time for calculations as well, but it is necessary to obtain accurate answers.

Run ANSYS-Fluent in 3D, Double Precision mode and follow the following instruction:

1- Read mesh file (e.g. 3dFullSymPropDuctF2.msh) from working directory

2- Solver: Type (Pressure-Based), Time (Transient)

3- Compile UDFs by going to Define $\rightarrow$ User Defined $\rightarrow$ Functions $\rightarrow$ Compiled and by choosing and loading 3 UDFs: Tilting.C, V-prop_inlet.C, and V-prop_outlet.C 
4- Mesh Rotate: Angle $=30$, Rotation Origin $(0,0,0)$, Rotation Axis $(0,0,1)$

5- Mesh scale: Scale the mesh by factor 0.22 in all directions to have the size of real ducts in the CFD simulations

6- Model: Choose RNG k-e Standard Wall fn for turbulence modeling

7- Material: $\rho=1.225 \mathrm{~kg} / \mathrm{m}^{3}$ and $\mu=1.7894 e-05 \mathrm{~kg} / \mathrm{ms}$

8- Boundary Conditions: Inlet, Velocity Inlet; Velocity Magnitude, $\mathrm{V}_{x}=15 \mathrm{~m} / \mathrm{s}$

Prop_inlet; Magnitude, Normal to boundary, choose UDF "V-prop_inlet"

Prop_outlet: Magnitude, Normal to boundary, choose UDF "V-prop_outlet"

9- Dynamic Mesh settings: select all zones and choose UDF "Tilting" to rotate all zones without any deformation in default interior cells during rotation of the duct in the simulation. Both location and orientation of the centre of gravity were set to $(0,0,0)$

10-Reference Values: Enter Area $=0.1520531 \mathrm{~m}^{2}$ for the area of duct. The other important parameter such as length $=0.22 \mathrm{~m}$ and velocity $=15 \mathrm{~m} / \mathrm{s}$ should be seen in the list. Choose Compute from Inlet.

11- Solution Method: In Spatial Discretization, choose Second Order Upwind for Momentum 12- Monitors: In Residual Monitors, deselect Plot and Print to Console and choose Absolute Criteria 0.001 for all Residuals (continuity, x-velocity, y-velocity, z-velocity, $\mathrm{k}$ and epsilon), setup files for recording aerodynamic coefficients

13- Solution Initialization: Choose Inlet for Compute From, then press Initialize button.

14- Run Calculation: Set Time Step Size $=0.01 \mathrm{sec}$, No. of Time Steps=1800 and Max Iterations/Time Step=100 and press Calculate button. 
Now ANSYS-Fluent starts working and will stop after finishing 1800 time steps. Results will be saved in cl-history, cd-history and cm-history files in the working directory. There are many other tools (like creating an Animation File or pictures for different properties of the fluid/case by using ANSYS-CFD Post) which readers can find in the ANSYS-Fluent and ANSYS-CFD Post manuals.

\section{User Defined Functions}

The ANSYS-Fluent software was used to compute lift, drag and pitching moment of tilting ducted fans in this research. Simulations were performed in stationary and transition conditions.

In stationary condition, the AoA and main stream velocity were set as constants and then the velocity of the inlet flow to rotor plane was set as another constant based on the selected AoA. The aerodynamic coefficients were recorded at the end of iterations for each AoA.

In transition condition, when ducted fans were tilting with specific tilting rate, all these were done automatically by using User Defined Functions (UDF). Time is the input variable to each UDf codes calculating tilting angle, main stream velocity and the velocity of the inlet flow to the rotor plane.

The following $\mathrm{C}++$ programs were used for defining tilting angle and the velocity of the inlet flow to the rotor plane for both symmetrical and asymmetrical ducted fans. The final aerodynamic coefficients were saved in three different files for each case.

Readers can change some parameters of these codes to conduct simulations for different tilting rate or different velocity for the inlet flow to the rotor plane. Readers should note that ANSYSFluent uses "Radians" for unit of angles to calculate trigonometric functions. More information about UDF files can be found in ANSYS-Fluent manual [21]. 


\section{1- UDF for calculating AoA in transition}

The tilting rate in this UDF is $\omega=10 \mathrm{deg} / \mathrm{sec}$. The initial setup for AoA is $\alpha=-30^{\circ}$. In the first 2 seconds after running the case (Time $\leq 2 \mathrm{sec}$ ), ducted fans do not have any tilting. For $2 \mathrm{sec} \leq$ Time $\leq 17 \mathrm{sec}$, the tilting rate is $\omega=10 \mathrm{deg} / \mathrm{sec}$ and AoA varies in the range of $-30^{\circ} \leq \alpha \leq 120^{\circ}$, which this transition takes 15 seconds. The additional 1 second time was used to record unsteady flow behaviour around ducted fan in high AoA $\left(\alpha=120^{\circ}\right)$.

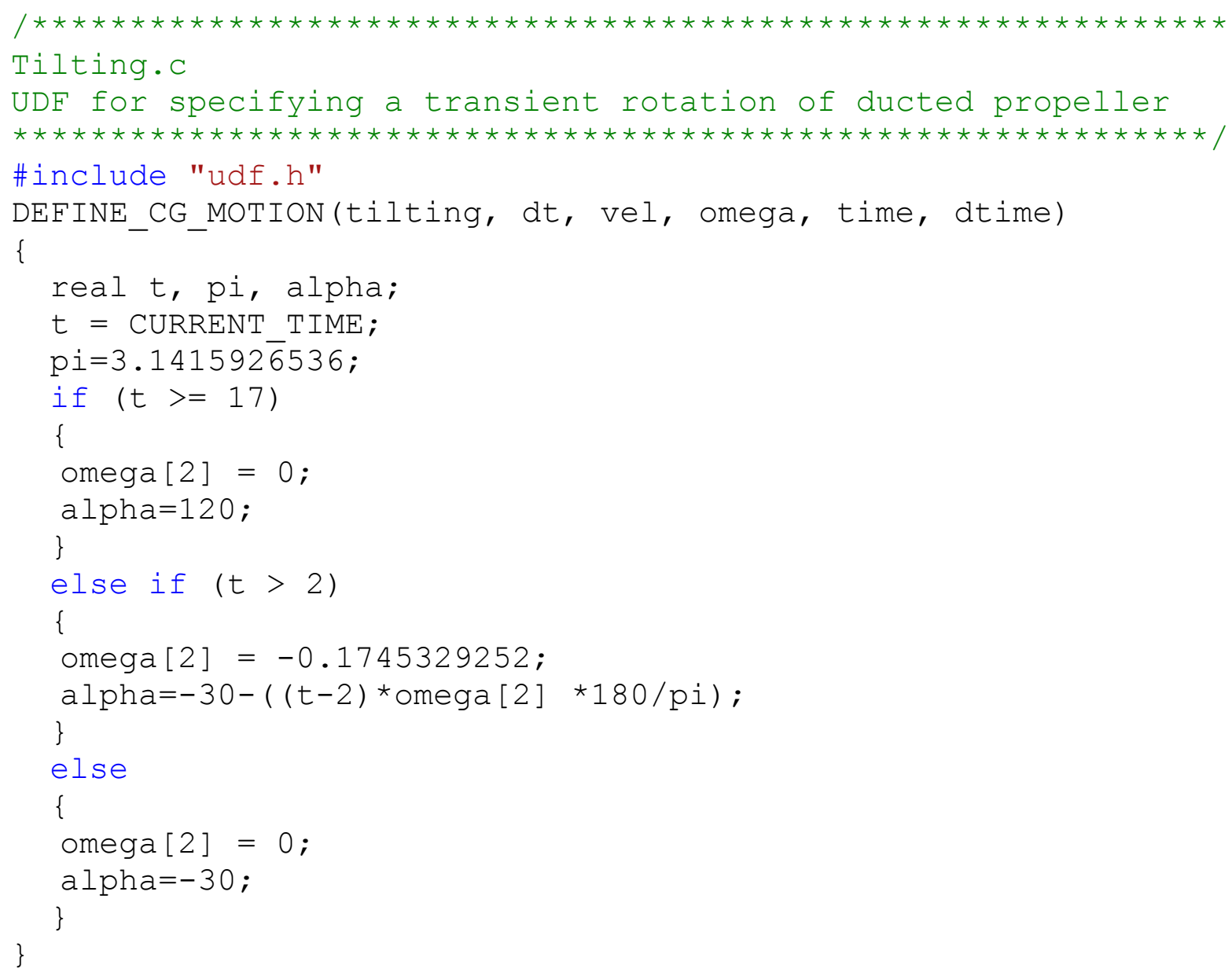

\section{2- UDF for calculating the velocity of the inlet flow to the rotor plane in transition}

In the first 2 seconds after running the case (Time $\leq 2 \mathrm{sec}$ ), ducted fans do not have any tilting and in this period the velocity of the inlet flow to the rotor plane is constant (e.g. for 
symmetrical ducted fan: $V_{\text {inlet }}=19.622 \mathrm{~m} / \mathrm{sec}$ and $\alpha=-30^{\circ}$ ). For $2 \mathrm{sec} \leq$ Time $\leq$ $17 \mathrm{sec}$, the velocity of the inlet flow to the rotor plane is being calculated based on the method explained in section 3.7. The additional 1 second time was used to record unsteady flow behaviour around ducted fan in high AoA $\left(\alpha=120^{\circ}\right.$ and $\left.V_{\text {inlet }}=9.852 \mathrm{~m} / \mathrm{sec}\right)$.

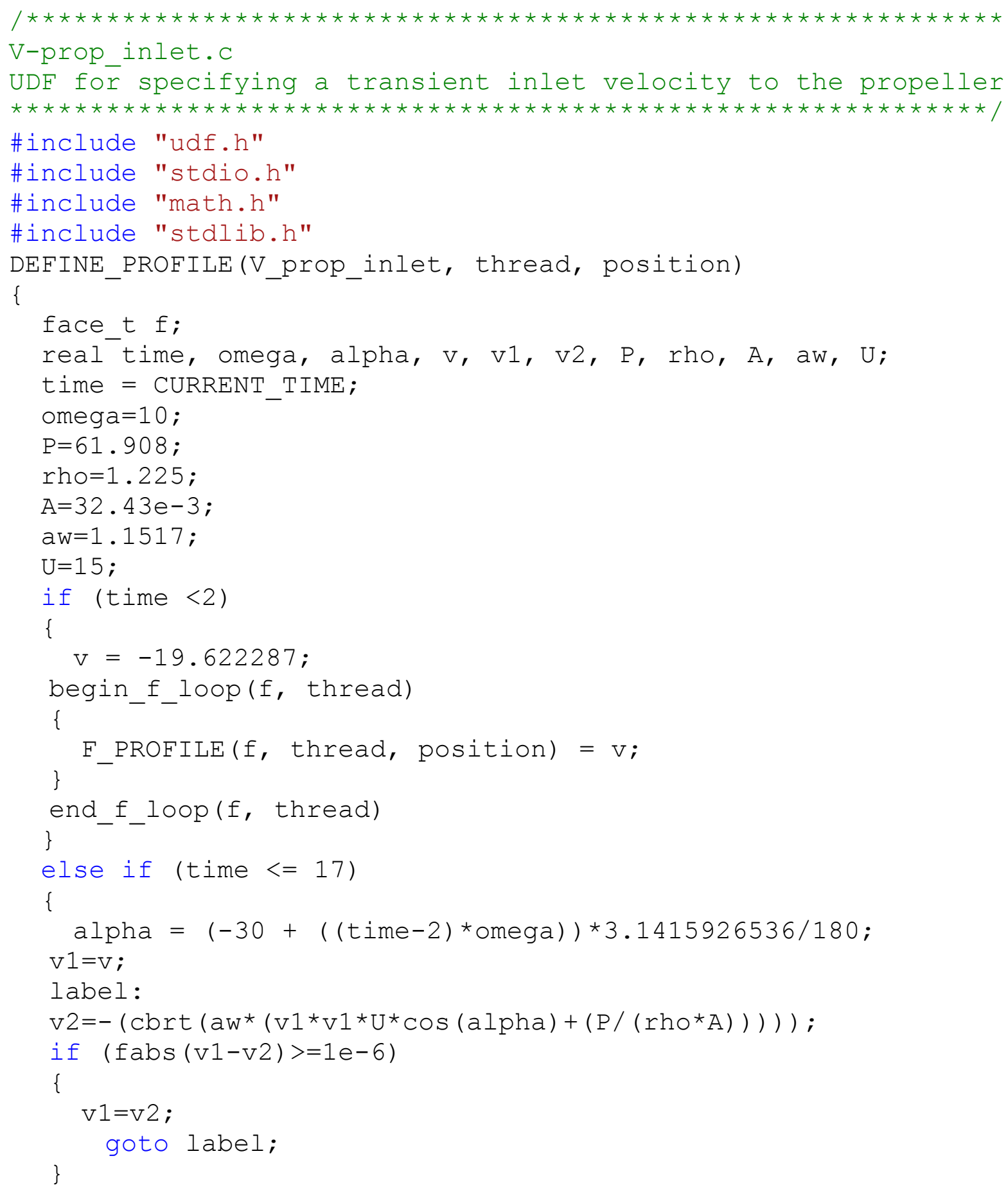




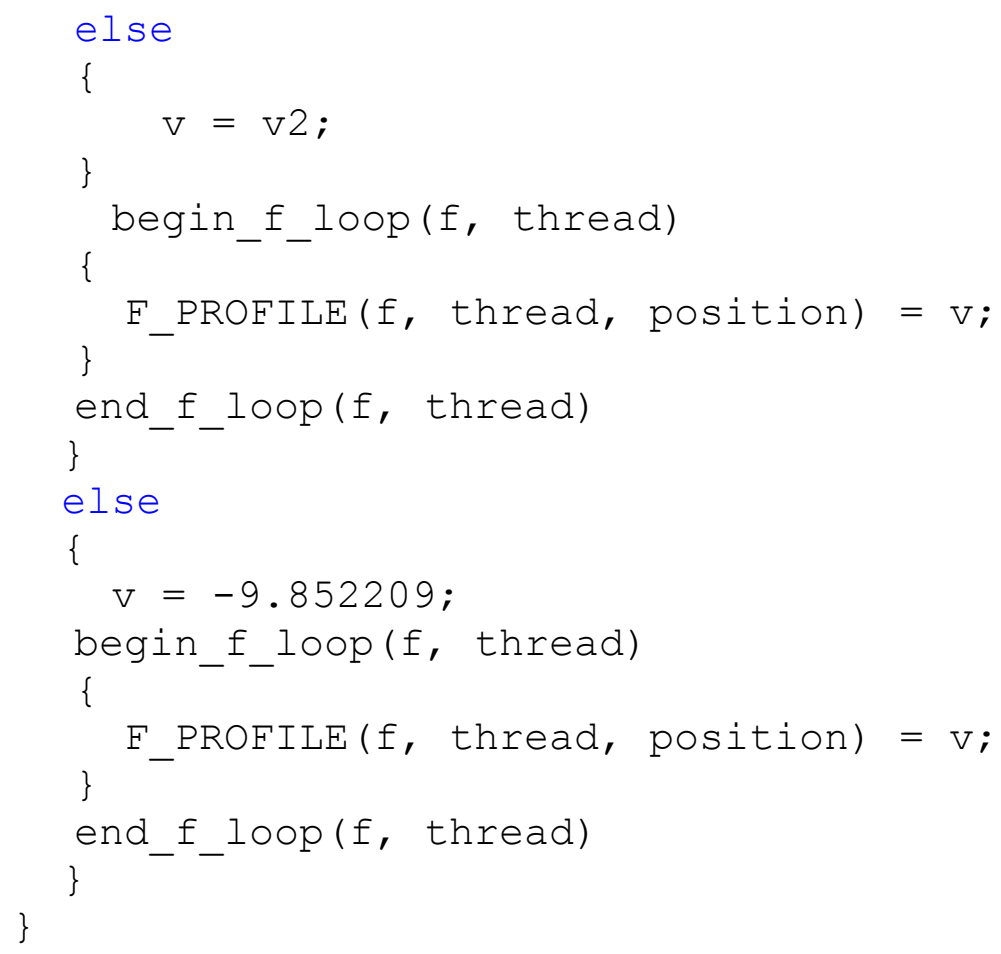

The negative sign for velocity shows inlet flow is going into the rotor plane. The UDF for outgoing flow from rotor plane has positive signs for velocities which shows flow coming out from the other side of the rotor plane. 


\section{Appendix I: The specifications of the proposed VTOL UAV with two ducted fans}

The proposed ducted fan VTOL UAV is shown in Figure I. 1. The calculation of the specification of the VTOL UAV can be started by an overview of the propeller specifications and the maximum weight of the VTOL UAV.

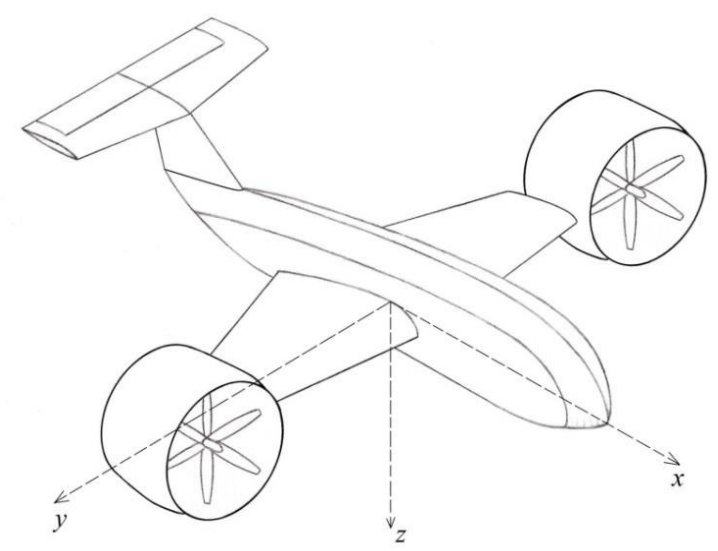

Figure I. 1: Proposed tilting ducted fan with airfoil shape fuselage

\section{Propeller, required thrust and maximum weight of the VTOL UAV}

The three-bladed propeller size is 8 " $\times 6$ " made by "Maser Airscrew Company". The maximum tip speed for this propeller is 600 to $650 \mathrm{ft} / \mathrm{sec}(183$ to $198 \mathrm{~m} / \mathrm{sec})$ and the maximum RPM for this propeller can be found as:

$R P M_{\max }=\frac{650 \times 12 \times 60}{8 \pi}=18,620$

The optimum RPM for this propeller can be calculated by the experimental formula by the "Master Airscrew Company" [33], which offers Mach 0.54 for the tip speed as follow:

$R P M_{\text {opt }}=\frac{138,000}{\text { prop. diameter }}=\frac{138,000}{8}=17,250 \quad$ (In the hovering)

The induced velocity into the rotating propeller for the hovering condition is:

$V_{\text {ind }_{\text {hover }}}=6 " \times 17,250=103,500 \mathrm{inch} / \mathrm{min}=43.815 \mathrm{~m} / \mathrm{sec}$ 
The maximum thrust in hovering condition is:

$T_{\text {hover }}=\frac{\rho A V_{\text {ind }}^{2}}{a_{w}}=\frac{1.225 \times \pi(4 \times 0.0254)^{2} \times 43.815^{2}}{1.1517}=66.2 \mathrm{~N}$

The wake contraction parameter was calculated before as: $a_{w}=\left(\frac{R_{o u t}}{R_{\text {in }}}\right)^{2}=1.1517$. The total thrust of two engines is $132.4 \mathrm{~N}$, which is enough in the hovering for a VTOL UAV with the mass of $13.5 \mathrm{~kg}$. The total induced power for generating the maximum required thrust is 5.8 kWatt.

As shown in Figure I. 1 two ducted fans are installed on the wing tips of the VTOL UAV. It is possible to use two different types of ducted fans (symmetrical or asymmetrical) for thrust generation. Based on the aerodynamic coefficients of these two ducted fans, the specification of the UAV can be discussed as follows:

\section{1- Using symmetrical ducted fans}

Each symmetrical ducted fan has the following specifications: $\bar{c}=0.22 \mathrm{~m}, \bar{d}=$ $0.22 \mathrm{~m}$, and $S=\pi d c=0.152053 \mathrm{~m}^{2}$. In the cruise condition $\left(\alpha=0^{\circ}\right.$ and $\left.U=15 \mathrm{~m} / \mathrm{s}\right)$, the aerodynamic coefficients were calculated by ANSY-Fluent as: $C_{l}=0, C_{d}=0.0127976$, and $C_{m}=0$, which resulted in: $L=0 \mathrm{~N}, D=0.268 \mathrm{~N} \& M=0 \mathrm{Nm}$.

The lift, drag and thrust of ducted fans can generate pitching moment about the cg of the UAV. The calculated thrust for symmetrical ducted fan in cruise condition is $2.913 \mathrm{~N}$. Assuming the tilting axis of the ducts are in line with the ac of the wing (which is designed to be on the top of the cg of the UAV with the vertical distance of $h_{a c}=0.04 \mathrm{~m}$ ), then drag and thrust of the ducted fans have pitching moments about the cg of the UAV as follows:

$M_{d u c t}=2\left(D_{d u c t}-T\right) h_{a c}=2(0.268-2.913)(0.04)=-0.2116 \mathrm{Nm}$ 
Finite wing: The high lift FX63-137 airfoil is used for designing the wing and by assuming $\mathrm{Re}=200,000$ for the cruise condition of the UAV. Although the maximum lift-to-drag ratio for this airfoil is: $\frac{C_{l}}{C_{d}}=90.64$ at $\alpha=6^{\circ}$, it's better to use incident angle of $\alpha=7^{\circ}$ to have greater lift. The aerodynamic coefficients of this airfoil at $\alpha=7^{\circ}$ are:

$\left(C_{l}\right)_{\alpha=7^{\circ}}=1.5900,\left(C_{d}\right)_{\alpha=7^{\circ}}=0.01778$, and $\left(C_{m}\right)_{\alpha=7^{\circ}}=-0.1867$

Assuming $A R=\frac{b^{2}}{S}=6$ and $e=0.95$, the effective AoA can be calculated as:

$\alpha_{e f f}=\alpha-\alpha_{i}=\alpha-\frac{57.3 C_{l}}{\pi e A R}=7^{\circ}-\frac{57.3 \times 1.59}{\pi(0.95)(6)}=7^{\circ}-5.1^{\circ}=1.9^{\circ}$

Which results in: $C_{L}=1.09184$ at $\alpha_{e f f}=1.9^{\circ}$.

The Reynolds no. $(\mathrm{Re}=200,000)$ can be used to estimate the chord length. Knowing the cruise speed as $U=15 \mathrm{~m} / \mathrm{s}$ results in:

$\bar{c}=\frac{\operatorname{Re} \mu}{\rho U}=\frac{200,000 \times 1.79 \times 10^{5}}{1.225 \times 15}=0.195 \mathrm{~m} \approx 0.2 \mathrm{~m} \mathrm{~m}$

And: $b=A R . c=6 \times 0.2=1.2 \mathrm{~m}$, which results in: $S=b c=0.24 \mathrm{~m}^{2}$

Then, the wing's lift is: $L=\frac{1}{2} \rho S U^{2} C_{L}=\frac{1}{2}(1.225)(0.24)(15)^{2}(1.09184)=36.1126 \mathrm{~N}$

Total lift in the cruise condition is equal to the weight of the VTOL UAV. Then, the mass of the VTOL UAV is:

$m=\frac{W}{g}=\frac{L_{w i n g}}{g}=\frac{36.1126}{9.806} \approx 3.683 \mathrm{~kg}$

Total drag coefficient is: $C_{D}=C_{d_{0}}+\frac{C_{L}^{2}}{\pi e A R}=0.01778+\frac{1.09184^{2}}{\pi(0.95)(6)}=0.08435$

Then, the wing's drag is:

$D=\frac{1}{2} \rho S U^{2} C_{D}=\frac{1}{2}(1.225)(0.24)(15)^{2}(0.08435)=2.79 \mathrm{~N}$

And pitching moment:

$M_{o}=\frac{1}{2} \rho S c U^{2} C_{m}=\frac{1}{2}(1.225)(0.24)(0.2)(15)^{2}(-0.1867)=-1.235 \mathrm{Nm}$ 
As the aerodynamic centre of the wing is on the top of the $\mathrm{cg}$ of the UAV, then the total pitching moment of the wing is:

$M_{\text {wing }}=M_{o}+D h_{a c}=-1.235+2.79 \times 0.04=-1.1234 \mathrm{Nm}$

Fuselage: The fuselage does not generate lift and pitching moment. Due to installation of some external components such as camera and communication devices, they will generate drag. Assuming $C_{d}=0.057$ at AoA $=0^{\circ}$ and fuselage sizes as: $b=0.2 \mathrm{~m}, c=1.5 \mathrm{~m}$, then $S=$ $0.3 \mathrm{~m}^{2}$ results in:

$D_{\text {fuselage }}=\frac{1}{2} \rho S U^{2} C_{d}=\frac{1}{2}(1.225)(0.3)(15)^{2}(0.057)=2.357 \mathrm{~N}$

There is no pitching moment due to the fuselage's drag as its aerodynamic center is in line with the cg of the UAV.

Vertical stabilizer: The airfoil for vertical stabilizer is NACA 0009 with the aerodynamic coefficients of: $C_{l}=0, C_{d}=0.0055$, and $C_{m}=0$ at $\alpha=0^{\circ}$. The specification for the vertical stabilizer are assumed as: $\bar{c}=0.082 \mathrm{~m}, b=0.245 \mathrm{~m}$, and $S=b c=0.02 \mathrm{~m}^{2}$, which results in:

$L=0 N, D=0.01516 N \& M=0 N m$

The drag of the vertical stabilizer has a pitching moment about the cg of the UAV. Assuming the vertical distance between the ac of the vertical stabilizer and the cg of the UAV as $h_{v s}=$ $0.09 \mathrm{~m}$, the pitching moment of the vertical stabilizer can be calculated as:

$M_{v s}=D_{v s} h_{v s}=(0.01516)(0.09)=0.001364 \mathrm{Nm}$

Horizontal stabilizer: The generated pitching moment from horizontal stabilizer should cancel the summation of the pitching moments from the other components about the cg of the VTOL UAV. Assuming the NACA 0009 airfoil for horizontal stabilizer with the initial specifications as: $\bar{c}=0.14 \mathrm{~m}, b=0.70 \mathrm{~m}, S=b c=0.098 \mathrm{~m}^{2}, e=0.9$ and the vertical and horizontal 
distances from the cg of the UAV as: $l_{h s}=-0.75 \mathrm{~m}$ and $h_{h s}=0.20 \mathrm{~m}$ gives the estimation for incidence angle for horizontal stabilizer. Then, for steady level flight: $\Sigma M_{o}=0$ yields to:

$M_{\text {wing }}+M_{d u c t}+M_{v s}+M_{h s}=0$

$M_{h s}=-[(-1.1234)+(-0.2116)+(0.001364)]=1.334 \mathrm{Nm}$

The horizontal stabiliser's lift coefficient can be calculated as:

$L_{h s}=\frac{M_{h s}}{l_{h s}}=\frac{1.334}{-0.75}=-1.779 \mathrm{~N}$

$C_{L}=\frac{2 L_{h s}}{\rho S V^{2}}=\frac{2(-1.779)}{1.225(0.098)\left(15^{2}\right)}=-0.1317$

Which happens at $\alpha_{e f f} \approx-1.3^{\circ}$. Using thin airfoil theory ( $C_{l}=2 \pi \alpha ; \alpha$ in Radians) results in:

$\alpha_{e f f}=\alpha-\alpha_{i}=\alpha-\frac{57.3 C_{l}}{\pi e A R}=\alpha-\frac{\left(57.3 \times \frac{\pi}{180}\right)(2 \pi \alpha)}{\pi e A R}=\alpha\left(1-\frac{2}{e A R}\right)$

$\alpha=\frac{\alpha_{e f f}}{1-\frac{2}{e A R}}=\frac{-1.3^{\circ}}{1-\frac{2}{(0.9)(5)}}=-2.34^{\circ}$

From the table of aerodynamic coefficients for NACA 0009 airfoil [34] at $\alpha=-2.34^{\circ}$ :

$C_{l}=-0.3$ and $C_{d}=0.0063$, and $C_{m}=0.004$

Then drag of the horizontal stabilizer can be calculated as follow:

$C_{D}=C_{d}+\frac{C_{L}^{2}}{\pi e A R}=0.0063+\frac{(-0.1317)^{2}}{\pi(0.9)(5)}=0.00753$

$D_{h s}=\frac{1}{2} \rho S V^{2} C_{D}=0.5(1.225)(0.098)(225)(0.00753)=0.1017 \mathrm{~N}$

The additional pitching moment of the horizontal stabilizer about the cg of the UAV due to its drag can be calculated as: 


$$
\begin{aligned}
M_{o}+M_{\text {drag }}= & \frac{1}{2} \rho S c V^{2} C_{m}+D_{h s} h_{h s} \\
& =0.5(1.225)(0.098)(0.14)(225)(0.004)+(0.1017)(0.2) \\
& =0.007563+0.002034=0.009597 \mathrm{~N}
\end{aligned}
$$

The horizontal stabilizer should generate $1 \%$ more lift to cancel this additional pitching moment. This reform changes incidence angle of the horizontal stabilizer to $\alpha=-2.36^{\circ}$

Thrust: Total thrust of the ducted fans should be equal to the total drag of the UAV in the cruise condition. The calculation shows:

$$
\begin{aligned}
& D_{\text {total }}=D_{\text {wing }}+D_{\text {fuselage }}+2 D_{\text {duct }}+D_{v s}+D_{\text {hs }} \\
& =2.79+2.357+2(0.268)+0.01516+0.1017=5.8 \mathrm{~N} \\
& T_{\text {total }}=2 T_{\text {dust }}=2(2.913)=5.826 \mathrm{~N}
\end{aligned}
$$

And the difference between two horizontal forces is $0.026 \mathrm{~N}$. The final specifications of the proposed VTOL UAV equipped with two symmetrical ducted fans are listed in the Table I. 1.

\section{2- Using asymmetrical ducted fans}

Each asymmetrical ducted fan has the following specifications: $\bar{c}=0.22 \mathrm{~m}, \bar{d}=$ $0.22 \mathrm{~m}$, and $S=\pi d c=0.152053 \mathrm{~m}^{2}$. In the cruise condition $\left(\alpha=0^{\circ}\right)$, the aerodynamic coefficients were calculated by ANSY-Fluent as: $C_{l}=0.09922, C_{d}=0.016948$, and $C_{m}=$ -0.014308 , which resulted in: $L=2.07914 \mathrm{~N}, D=0.35514 \mathrm{~N} \& M=-0.066 \mathrm{Nm}$.

The lift, drag and thrust of ducted fans can generate pitching moment about the cg of the UAV. The calculated thrust for asymmetrical ducted fan in cruise condition is $3.045 \mathrm{~N}$. Assuming the tilting axis of the ducts are in line with the ac of the wing, then drag of ducted fans have pitching moments about the cg of the UAV as follows: 
$M_{\text {ducts }}=2\left[M_{o}+\left(D_{\text {duct }}-T\right) h_{a c}\right]=2[-0.066+(0.35514-3.045)(0.04)]=$ $-0.3472 \mathrm{Nm}$

Finite wing: The wing has to generate less lift as follows:

$L_{w}=W-2 L_{d u c t}=36.1126-2(2.07914)=31.9543 \mathrm{~N}$

Assuming the same aerodynamic coefficients for the wing similar to the symmetrical ducted fan case, the area of the wing can be calculated as:

$S=\frac{2 L}{\rho U^{2} C_{L}}=\frac{2(31.9543)}{(1.225)(15)^{2}(1.09184)}=0.21236 \mathrm{~m}^{2}$

Assuming the same aspect ratio yields to: $\mathrm{b}=1.129 \mathrm{~m}$ and $\mathrm{c}=0.188 \mathrm{~m}$. Then by using asymmetrical ducted fans, it is possible to use a wing with $11.5 \%$ smaller surface area than that for symmetrical ducted fan. The reduction in the wing area will reduce the wing's drag and pitching moment as following:

$D=\frac{1}{2} \rho S U^{2} C_{D}=\frac{1}{2}(1.225)(0.21236)(15)^{2}(0.08435)=2.4686 \mathrm{~N}$

And pitching moment:

$M_{o}=\frac{1}{2} \rho S c U^{2} C_{m}=\frac{1}{2}(1.225)(0.21236)(0.188)(15)^{2}(-0.1867)=-1.0272 \mathrm{Nm}$

As the aerodynamic centre of the wing is on the top of the cg of the UAV, then the total pitching moment of the wing is:

$M_{\text {wing }}=M_{o}+D h_{a c}=-1.0272+2.4686 \times 0.04=-0.9285 \mathrm{Nm}$

Fuselage: The fuselage does not generate lift and pitching moment. Due to installation of some external components such as camera and communication devices, they will generate drag. Assuming $C_{d}=0.0677$ at AoA $=0^{\circ}$ and fuselage sizes as: $b=0.2 \mathrm{~m}, c=1.5 \mathrm{~m}$, then $S=$ $0.3 \mathrm{~m}^{2}$ results in: 
$D_{\text {fuselage }}=\frac{1}{2} \rho S U^{2} C_{d}=\frac{1}{2}(1.225)(0.3)(15)^{2}(0.0677)=2.8 \mathrm{~N}$

There is no pitching moment due to the fuselage's drag as its aerodynamic center is in line with the cg of the UAV.

Vertical stabilizer: The airfoil for vertical stabilizer is NACA 0009 with the aerodynamic coefficients of: $C_{l}=0, C_{d}=0.0055$, and $C_{m}=0$ at $\alpha=0^{\circ}$. The specification for the vertical stabilizer are assumed as: $\bar{c}=0.082 \mathrm{~m}, b=0.245 \mathrm{~m}$, and $S=b c=0.02 \mathrm{~m}^{2}$, which results in:

$L=0 N, D=0.01516 N \& M=0 N m$

The drag of the vertical stabilizer has a pitching moment about the cg of the UAV. Assuming the vertical distance between the ac of the vertical stabilizer and the cg of the UAV as $h_{v s}=$ $0.09 \mathrm{~m}$, the pitching moment of the vertical stabilizer can be calculated as:

$M_{v S}=D_{v s} h_{v s}=(0.01516)(0.09)=0.001364 \mathrm{Nm}$

Horizontal stabilizer: The generated pitching moment from horizontal stabilizer should cancel the summation of the pitching moments from the other components about the cg of the VTOL UAV. Assuming the NACA 0009 airfoil for horizontal stabilizer with the initial specifications as: $\bar{c}=0.14 m, b=0.70 m, S=b c=0.098 m^{2}, e=0.9$ and the vertical and horizontal distances from the cg of the UAV as: $l_{h s}=-0.75 \mathrm{~m}$ and $h_{h s}=0.20 \mathrm{~m}$ gives the estimation for incidence angle for horizontal stabilizer. Then, for steady level flight: $\Sigma M_{o}=0$ yields to:

$$
\begin{aligned}
& M_{\text {wing }}+2 M_{d u c t}+M_{v s}+M_{h s}=0 \\
& M_{h s}=-[(-0.9285)+(-0.3472)+(0.001364)]=1.274336 \mathrm{Nm}
\end{aligned}
$$

The horizontal stabiliser's lift coefficient can be calculated as:

$$
L_{h s}=\frac{M_{h s}}{l_{h s}}=\frac{1.274336}{-0.75}=-1.7 \mathrm{~N}
$$


$C_{L}=\frac{2 L_{h s}}{\rho S V^{2}}=\frac{2(-1.7)}{1.225(0.098)\left(15^{2}\right)}=-0.1259$

Which happens at $\alpha_{e f f} \approx-1.2^{\circ}$. Using thin airfoil theory $\left(C_{l}=2 \pi \alpha ; \alpha\right.$ in Radians $)$ results in:

$\alpha_{e f f}=\alpha-\alpha_{i}=\alpha-\frac{57.3 C_{l}}{\pi e A R}=\alpha-\frac{\left(57.3 \times \frac{\pi}{180}\right)(2 \pi \alpha)}{\pi e A R}=\alpha\left(1-\frac{2}{e A R}\right)$

$\alpha=\frac{\alpha_{e f f}}{1-\frac{2}{e A R}}=\frac{-1.2^{\circ}}{1-\frac{2}{(0.9)(5)}}=-2.16^{\circ}$

From the table of aerodynamic coefficients for NACA 0009 airfoil [34] at $\alpha=-2.16^{\circ}$ :

$C_{l}=-0.25$ and $C_{d}=0.006$, and $C_{m}=0.004$

Then drag of the horizontal stabilizer can be calculated as follow:

$C_{D}=C_{d}+\frac{C_{L}^{2}}{\pi e A R}=0.006+\frac{(-0.1259)^{2}}{\pi(0.9)(5)}=0.00712$

$D_{h s}=\frac{1}{2} \rho S V^{2} C_{D}=0.5(1.225)(0.098)(225)(0.0712)=0.0962 \mathrm{~N}$

The additional pitching moment of the horizontal stabilizer about the cg of the UAV due to its drag can be calculated as:

$$
\begin{aligned}
M_{o}+M_{\text {drag }}= & \frac{1}{2} \rho S c V^{2} C_{m}+D_{h s} h_{h s} \\
& =0.5(1.225)(0.098)(0.14)(225)(0.004)+(0.0962)(0.2) \\
& =0.007563+0.01924=0.026803 \mathrm{~N}
\end{aligned}
$$

The horizontal stabilizer should generate $2 \%$ more lift to cancel this additional pitching moment. This reform changes incidence angle of the horizontal stabilizer to $\alpha=-2.2^{\circ}$

Thrust: Total thrust of the ducted fans should be equal to the total drag of the UAV in the cruise condition. The calculation shows:

$$
\begin{aligned}
D_{\text {total }}=D_{\text {wing }} & +D_{\text {fuselage }}+2 D_{\text {duct }}+D_{v s}+D_{\text {hs }} \\
& =2.4686+2.8+2(0.35514)+0.01516+0.0962=6.09 \mathrm{~N}
\end{aligned}
$$


$T_{\text {total }}=2 T_{\text {dust }}=2(3.045)=6.09 \mathrm{~N}$

The final specifications of the proposed VTOL UAV equipped with two symmetrical ducted fans are listed in the Table I. 1.

Table I. 1: Specifications of the proposed VTOL UAV equipped with two symmetrical (or by using asymmetrical) ducted fans

Overall length

Wing area

Wing span (tip to tip)

Mean chord

Root chord

Tip chord

Wing's taper ratio $\left(c_{\text {root }} / c_{\text {tip }}\right)$

Wing's incidence angle

Wing's airfoil type

Span, between prop centers

Span, max. (with propellers)

Horizontal stabilizer's area

Horizontal stabilizer incidence angle

Vertical stabilizer's area

H \& V stabilizers airfoil type
$1.50 \mathrm{~m}$

$0.24 \mathrm{~m}^{2}$

$\left(0.21236 \mathrm{~m}^{2}\right.$ by using asymmetrical ducted fans)

$1.20 \mathrm{~m}$

(1.129 $\mathrm{m}$ by using asymmetrical ducted fans)

$0.24 \mathrm{~m}$

( $0.188 m$ by using asymmetrical ducted fans)

$0.36 \mathrm{~m}$

( $0.282 m$ by using asymmetrical ducted fans)

$0.12 \mathrm{~m}$

(0.094 $m$ by using asymmetrical ducted fans) 3

$7^{\circ}$

FX 63-137

$1.42 \mathrm{~m}$

(1.349 $\mathrm{m}$ by using asymmetrical ducted fans)

$1.64 \mathrm{~m}$

(1.569 $\mathrm{m}$ by using asymmetrical ducted fans)

$0.098 \mathrm{~m}^{2}$

$-2.36^{\circ}$

$\left(-2.2^{\circ}\right.$ by using asymmetrical ducted fans)

$0.02 \mathrm{~m}^{2}$

NACA0009 
Flying weight, max.

Drag of fuselage, max.

Propeller diameter:

Disk loading, max.

Propeller speed in the hover

Cruise velocity

Thrust (each rotor in airplane mode)

Thrust (each rotor in the hover)

Induced velocity in hovering (for each rotor)

Power consumption in hovering (by each rotor)

Rotors tilting angle range, max.

Rotors tilting range for directional control in hover $\left(\alpha=90^{\circ}\right)$

Rotors tilting range for longitudinal control in cruise mode $\left(\alpha=0^{\circ}\right)$

Rotor's tilting rate

Propellers
$3.683 \mathrm{~kg}$

$2.357 \mathrm{~N}$

(2.8 $\mathrm{N}$ by using asymmetrical ducted fans)

$0.203 \mathrm{~m}$ (8 in.)

$556.8 \mathrm{~N} / \mathrm{m}^{2}$

9000 RPM

$15 \mathrm{~m} / \mathrm{sec}$

$2.913 \mathrm{~N}$

(3.045 $\mathrm{N}$ by using asymmetrical ducted fans)

$18.058 \mathrm{~N}$

$22.88 \mathrm{~m} / \mathrm{s}$

413.17 Watt

$-10^{\circ} \leq \alpha \leq 120^{\circ}$

$\Delta \alpha= \pm 10^{\circ}$

$\Delta \alpha= \pm 10^{\circ}$

$\omega=10,20 \& 30 \mathrm{deg} / \mathrm{sec}$

Master Airscrew 8" × 6" 3-bladed, fixed pitch, (1) CW \& (1) CCW 


\section{Appendix J: MATLAB code for calculating flight parameters of proposed VTOL UAV during transition between cruise mode to hover}

The following MATLAB code was used to calculate 9 parameters during transition between cruise mode and hover of the proposed VTOL UAV as follows:

1- Ducted fans parameters: Tilting angle $(\alpha)$, Thrust (T), Induced velocity to the rotor plane $\left(V_{\text {ind }}\right)$, and Induced power to the rotor $(\mathrm{P})$.

2- Dynamic parameters of VTOL UAV: Travelled distance (x), Flight velocity (U), Braking deceleration $\left(a_{x}\right)$, Changes in altitude (z), and Transitional pitching moment.

The specification of VTOL UAV (see Table I. 1) with two symmetrical ducted fans installed on its wing tips were used to obtain this code. The reader can change tilting rate or type of ducted fan to calculate those 9 parameters in other conditions. The aerodynamic coefficients of tilting symmetrical ducted fan with tilting rate of $\omega=10 \mathrm{deg} / \mathrm{sec}$ were stored in "Symduct10.txt" file and were used in the following MATLAB code.

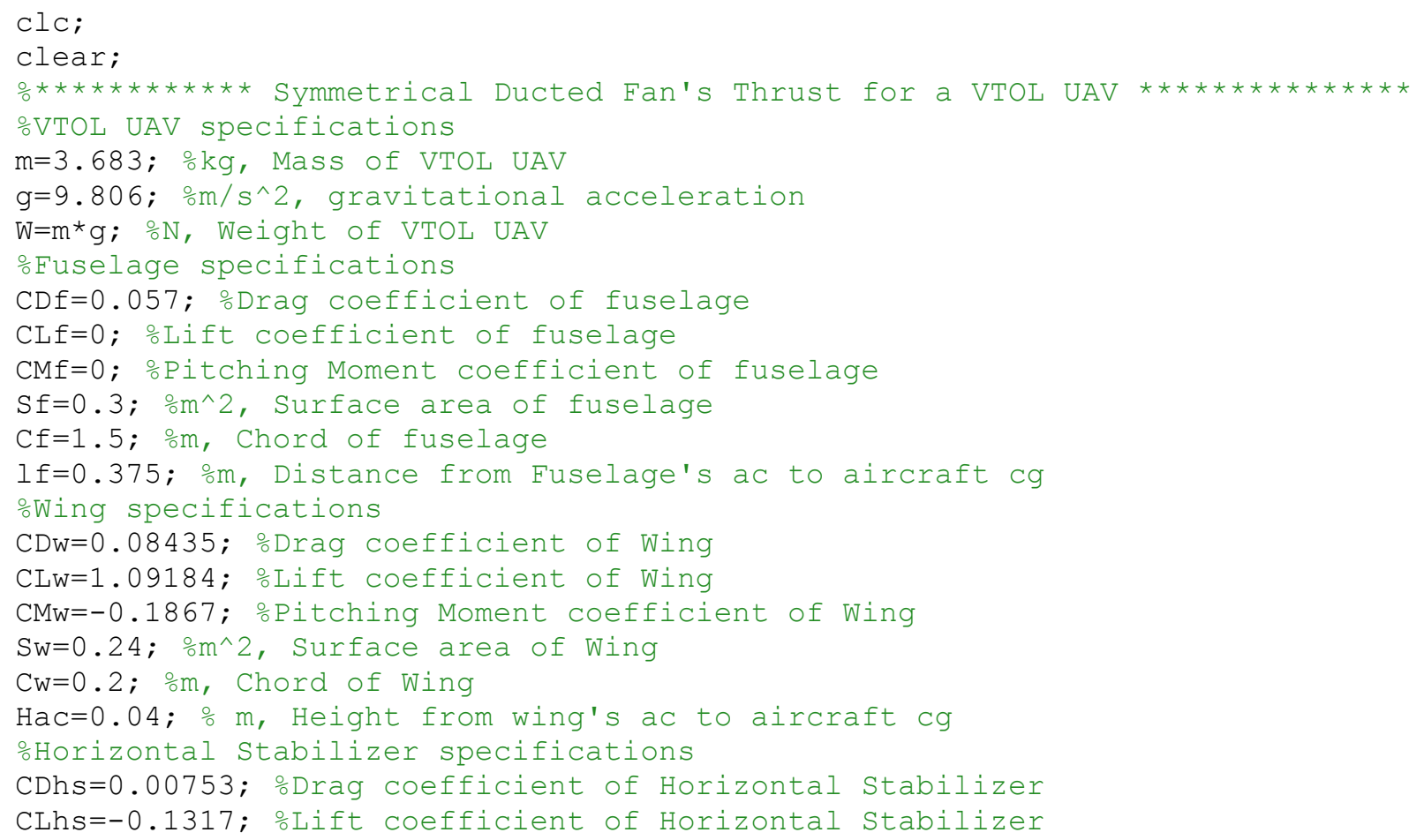




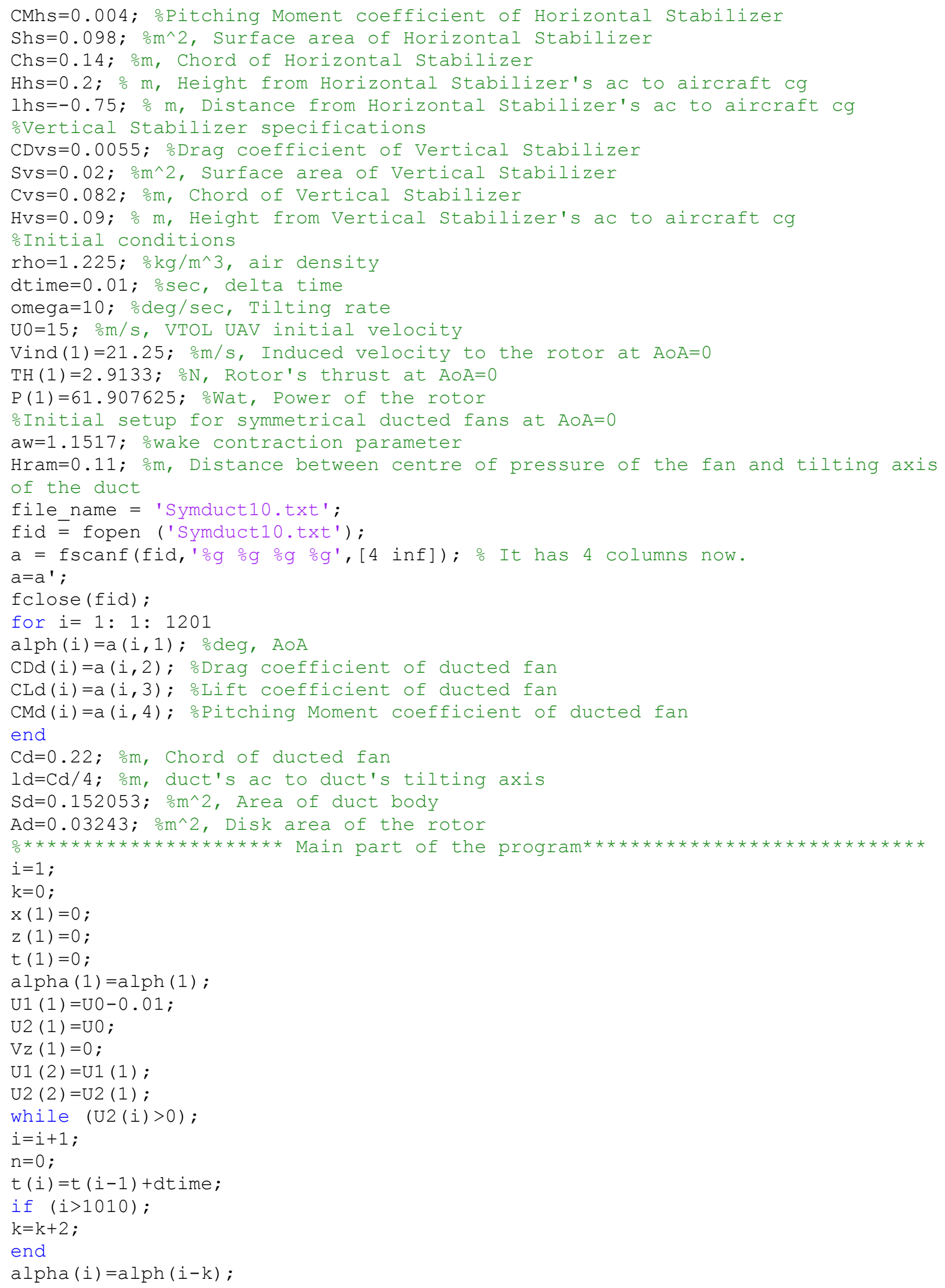




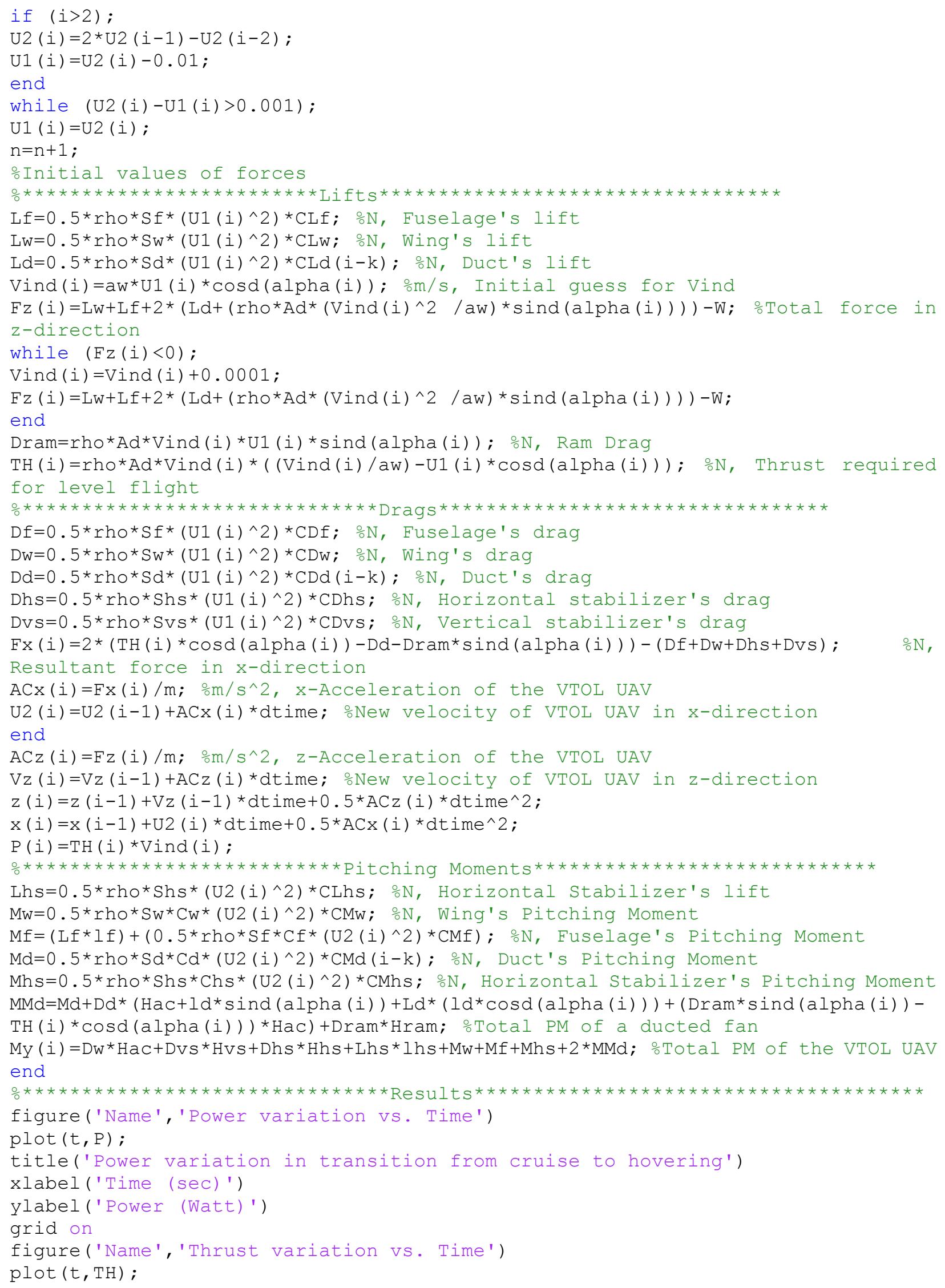




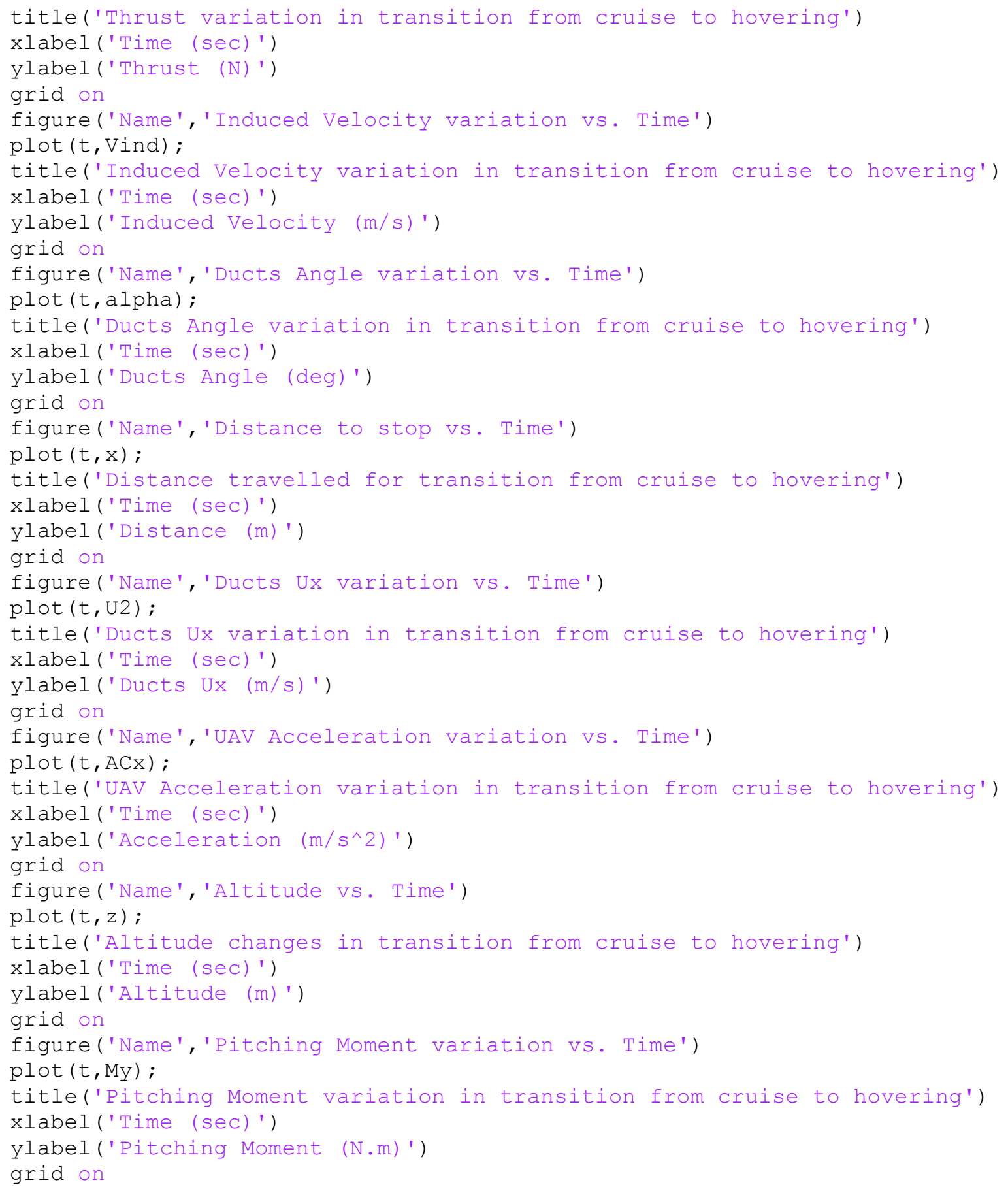




\section{REFERENCES}

[1] Image Attribution: Yamaha RMAX, Yamaha Motor Australia website: http://rmax.yamahamotor.com.au/specifications, July 22, 2016

[2] McCormick, B. W., Aerodynamics of V/STOL Flight, Dover Publications, 1999

[3] Image Attribution: AVID's technology website: http://www.avidaerospace.com/t-hawk-mav/, July 22,2016

[4] Image Attribution: BAE VTOL UAV impresses in autonomous tests, Gizmag website: http://www.gizmag.com/go/4539/, July 22, 2016

[5] Image Attribution: Xconomy website: http://www.xconomy.com/boston/2007/10/09/a-visitto-bostons-own-robot-plane-skunk-works/\#, July 22, 2016

[6] Gress, Gary R., "Lift Fans as Gyroscopes for Controlling Compact VTOL Air Vehicles: Overview and Development Status of Oblique Active Tilting", 63rd Annual Forum of the American Helicopter Society, Virginia Beach VA, May 1-3 2007

[7] Hirschberg, Mike, "German V/STOL Rotorcraft and Propellercraft Designs of the Twentieth Century", AHS Forum 57, STOVL Technology Session, Washington DC, 11 May 2001

[8] Martin, P. and Tung, C., "Performance and Flowfield Measurements on a 10-inch Ducted Rotor VTOL UAV", 60th Annual Forum of the American Helicopter Society, Baltimore, MD, June 710, 2004.

[9] Fleming J., Jones, T., Gelhausen, P., Enns, D., "Improving Control System Effectiveness for Ducted Fan VTOL UAVs Operating in Crosswinds" $2^{\text {nd }}$ AIAA "Unmanned Unlimited Systems, Technologies, and Operations-Aerospace, San Diego, CA, Sep 15-18, 2003

[10] Graf, W., Fleming, J., Wing, Ng., "Improving Ducted Fan UAV Aerodynamics in Forward Flight" 46th AIAA Aerospace Sciences Meeting and Exhibit, Reno, Nevada, Jan 2008

[11] Akturk, A., Shavalikul, A., Camci, C., "PIV Measurements and Computational Study of a 5Inch Ducted Fan for V/STOL UAV Applications" 47th AIAA Aerospace Sciences Meeting and Exhibit, Orlando, Florida, January 2009

[12] Camci, C. and Akturk, A., 2010, "Double Ducted Fan” The Pennsylvania State University, A technology description document. 
[13] Mort, K. W. and Gamse, B., 1967, “A wind tunnel investigation of a 7-foot diameter ducted propeller", Tech. Rep. NASA TND-4142.

[14] Yaggy, P. F. and Mort, K. W., 1961, “A wind-tunnel investigation of a 4-foot-diameter ducted fan mounted on the tip of a semispan wing”, Tech. Rep. NASA TND-776.

[15] Mort, K. W. and Yaggy, P. F., 1962, "Aerodynamic characteristics of a 4-foot-diameter ducted fan mounted on the tip of a semispan wing”, Tech. Rep. NASA TND-1301.

[16] Weir, R. J., 1988, “Aerodynamic design consideration for a free flying ducted propeller”, AIAA Atmospheric Flight Mechanics Conference.

[17] Abrego, A., I. and Bulaga, R., W., "Performance Study of a Ducted Fan System", AHS Aerodynamics, Aeroacoustic, Test and Evaluation Technical Specialist Meeting, San Francisco, CA, Jan 2002

[18] Leishman, J.G., 2006. Principles of Helicopter Aerodynamics, second ed. Cambridge University Press, London.

[19] Houghton, E.L., Carpenter, P.W., Collicott, S.H., Valentine, D.T., 2013. Aerodynamics for Engineering Students, sixth ed. Elsevier, Oxford.

[20] D.M. Somers and M.D. Maughmer, "Theoretical Aerodynamic Analyses of Six Airfoils for Use on Small Wind Turbines" 2003

[21] ANSYS Fluent tutorial, 2013

[22] Ko, A., Ohanian, O.J., Gelhausen, P., "Ducted Fan UAV Modeling and Simulation in Preliminary Design" AIAA Modeling and Simulation Technologies Conference and Exhibit, Hilton Head, South Carolina, August 2007

[23] Pereira, Jason L. "Hover and Wind-Tunnel Testing of Shrouded Rotors for Improved Micro Air Vehicle Design”. Ph.D. dissertation. University of Maryland, 2008. Print.

[24] B. E. Launder and D. B. Spalding. Lectures in Mathematical Models of Turbulence. Academic Press, London, England, 1972.

[25] V. Yakhot and S. A. Orszag. Renormalization Group Analysis of Turbulence: I. Basic Theory. Journal of Scientific Computing, 1(1):1-51, 1986.

[26] Bearman, P.W., "Vortex Shedding from Oscillating Bluff Bodies" Annual Review of Fluid Mechanics. 1984. 16: 195-222

[27] Williamson, C.H.K.; Govardhan, R., "Vortex-induced vibrations", Annual Review of Fluid Mechanics.2004. 36: 413-455 
[28] P. Spalart and S. Allmaras. "A one-equation turbulence model for aerodynamic flows". Technical Report AIAA-92-0439. American Institute of Aeronautics and Astronautics. 1992.

[29] D. C.Wilcox. Turbulence Modeling for CFD. DCW Industries, Inc. La Canada, California. 1998.

[30] White, F.M., 1991. Viscous Fluid Flow, $2^{\text {nd }}$ ed. McGraw-Hill, New York.

[31] Rosen, Aaron M. "Turbulence Modeling for Subsonic Separated Flows Over 2-D Airfoils and 3-D Wings". Ph.D. dissertation. Purdue University, 2013. Print.

[32] Taylor, J.R., 1997. An Introduction to Error Analysis: The Study of Uncertainties in Physical Measurements, $2^{\text {nd }}$ ed. Sausalito, CA: University Science Books.

[33] Master Airscrew, "The basics of propellers", Republished in the RC Groups Forums, www.rcgroups.com/forums/showatt.php?attachmentid=5383707, July 22, 2016

[34] Anderson, J.D., 2012. Introduction to Flight, $7^{\text {th }}$ ed. McGraw-Hill, New York. 